\title{
Problemas de Otimização Linear Canalizados e Esparsos ${ }^{*}$
}

Carla Taviane Lucke da Silva

Orientadora: Prof. Dr. Marcos Nereu Arenales

Dissertação apresentada ao Instituto de Ciencias Matcmáticas e de Computação - ICMC-lISP. como parte dos requisitos para obtenção do título de Mestre em Ciências de Computação e Matemática Compulacional.

USP - São Carlos

Novembro de 2002

"Fste traballer contou com o apoio da FAPFSP 


\section{A Comissão Julgadora:}

Prof. Dr. Marcos Nereu Arenales.

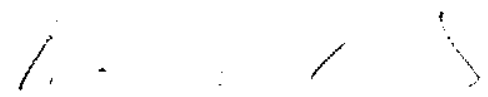

Prof. Ir. Lrneste Julian Goldherg Birgin

Prof. Dr. Lais (instavo Nonato

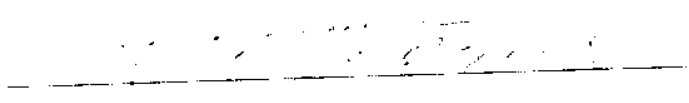


Ao Edson

con muito amor. 


\section{Agradecimentos}

Neste momento, quero agradecer as pessoas que de diversas maneiras contribuíram para a realização deste trabalho:

Ao Edson, esposo companheiro e dedicado. que me ajudou muito durante todo o tempo.

* Nos meus pais Rosimir e Elisabeth que me apoiaram e me incentivaram durante o desenvolvimento deste trabalho.

* No Marcos Arenales pela comprecnsão e simpatia, além de suas importantes orientações.

* A Selma Arenales pela confiança, incentivo e amizade.

* Ao Ricardo pela amizade e ajuda dada no decorrer deste trabalho.

* A FAPESP e ao CNPq pelo apoio financeiro.

- A Deus por permitir a conclusão deste trabalho e pela força dada, principalmente nos momentos mais difíceis. 


\section{Sumário}

Capítulo 1 - Introdução ..............................................................................

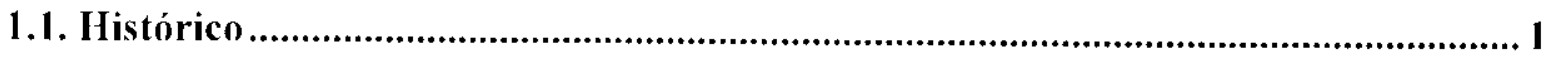

Capítulo 2 - Dualidade.................................................................................5

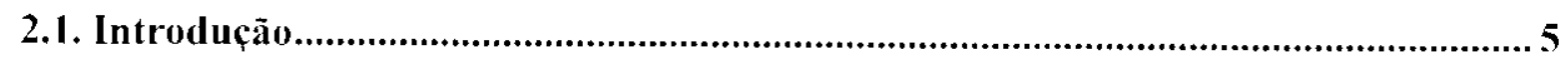

2.2. Relaxação Lagrangiana e o problema dual .................................................................5

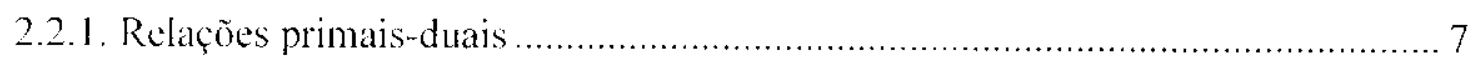

2.3. Problemas de otimização linear com restrições e variáveis canalizadas................... 10

2.3.1. O problema de otimização linear com restrições canalizadas ............................ 11

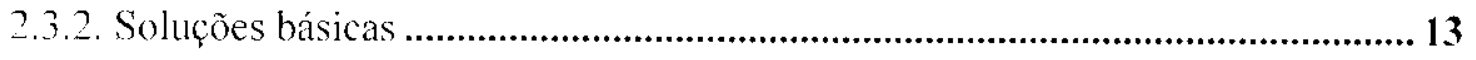

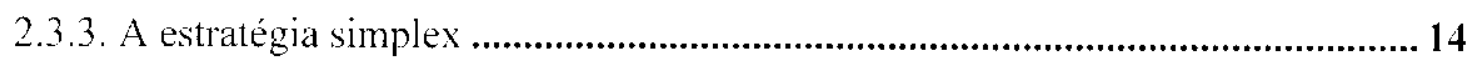

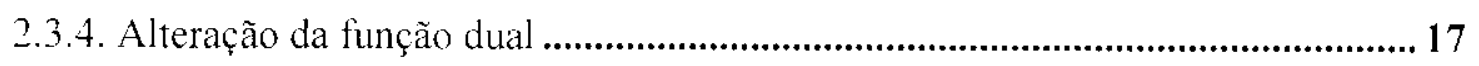

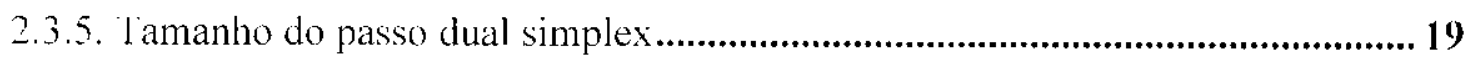

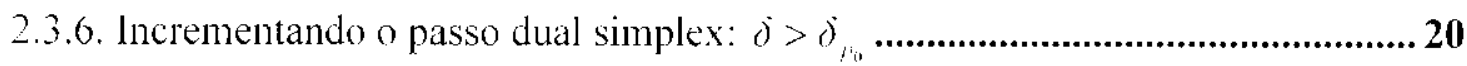

2.3.7. Tamanho do incremento do passo dual simplex ................................................25

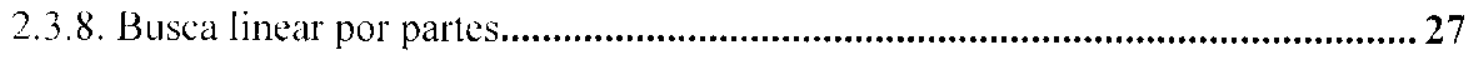

2.4. Método dual simplex canalizado com busca linear por partes .............................. 28

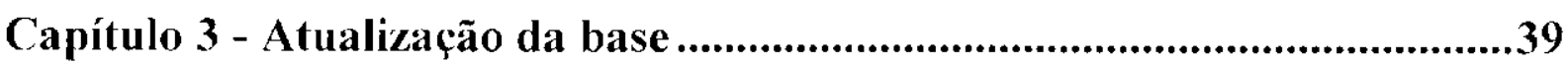

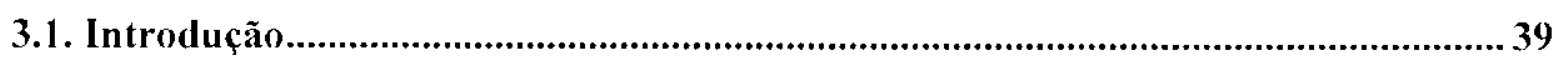

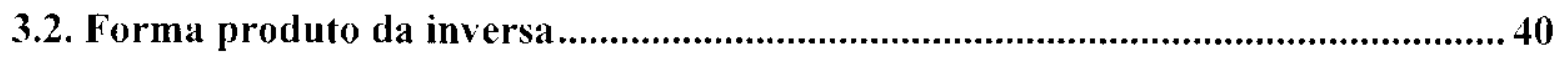

3.3. Decomposição LU de Bartels-Golub..................................................................43

3.4. Resolução de sistemas lineares com atualização da decomposição Ll ...................50

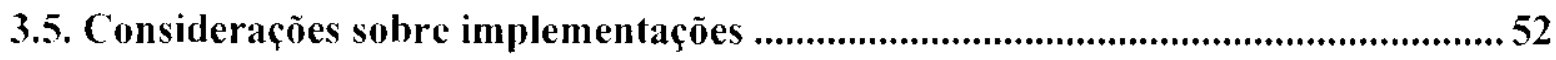

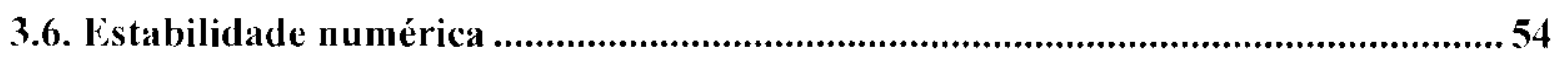

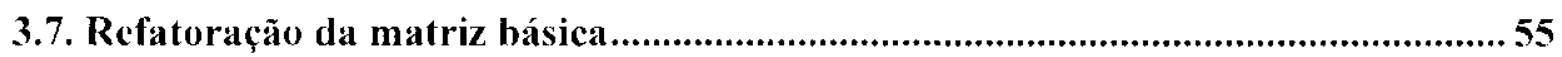


Capítulo 4 - Atualização da base: Esparsidade 58

4.1. Introdução.............................................................................................................. 58

4.2. Forma climinação da inversa ………………………......................................... 58

4.2.1. Descrição do mélodo ...........................................................................................59

4.3. Variantes de Bartels-Golub .................................................................................65

4.3.1. Variante 1 - Algoritmo esparso de Bartels-Golub ..................................................66

4.3.2. Variante 2 - $\Lambda$ perfeiçoamento do algoritmo esparso de Bartels-Golub ...............74

Capítulo 5 - Heurísticas de Pivoteamento ........................................................83

5.1. Introdução

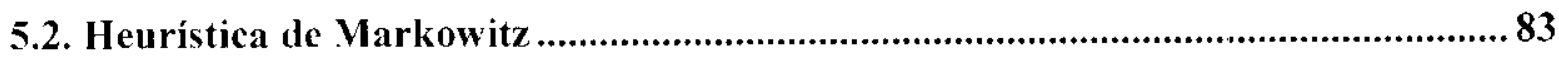

5.3. Heurística de Grau Mínimo .........................................................................................8

Capítulo 6 - Experimentos Computacionais .....................................................94

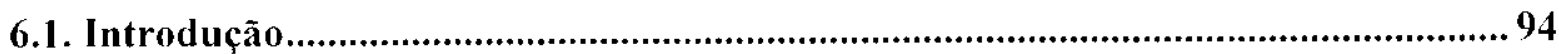

6.2. Estrutura de dados..............................................................................................94

6.2.1. Descrição da estrutura de dados ........................................................................ 95

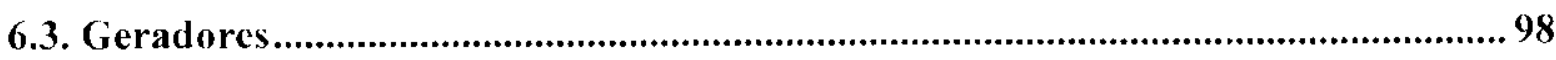

6.4. Um pouco sobre o programa ...................................................................................... 104

6.5. Resultados computacionais ................................................................................... 105

Capítulo 7 - Conclusões e Propostas Futuras.....................................................144

7.1. Conclusões............................................................................................................. 144

7.2. Propostas Futuras ................................................................................................. 146

Referências Bibliográficas...............................................................................147 


\section{Resumo}

A otimização linear tem sido objeto de estudo desde a publicaçẫo do método simplex em 1947. o qual vem sendo utilizado na prática com relativa eficiencia. Com isso, inúmeras variantes deste método surgiram na tentativa de se obter métodos mais eficientes. além de várias implementações objetivando a resolução de problemas de grande porte.

Os problemas de otimização linear canalizados e esparsos, objeto principal deste trabalho. são problemas de grande interesse prático. pois representan vários problemas reais, como por exemplo, problemas da programação da produção, problcmas de mistura e muitos outros.

O método dual simplex canalizado com busca linear por partes é um método do tipo simplex especializado para os problemas de otimização linear canalizados e será detalhado neste trabalho. Ixperiências computacionais toram realizadas para algumas classes de problemas de otimização linear com o objetivo de analisar o desempenho deste método, o qual foi implementado com algumas heurísticas de pivoteamento e formas de atualização da matriz básica para tentar manter a esparsidade presente e reduzir o tempo de resolução dos problemas. 
Lincar optimization has been studied sinee 1947 when the simplex method was published by George Dantrig. and it is still sucecsslully used in practice. $\Lambda$ number of variants to the simplex method have been proposed trying to obtain better efficiency. In addition, various implementations have been proposed to deal with large scale problems.

Two-side constraints and sparse linear optimization problems, the main object of this work, are of great interest in practice, since they represent a number of real problems, such as. production planning problems. mix problems and others.

This work presents a simplex-typed method, named two-side constraint dual simplex method with piecewise linear search. This method was implemented together with some heuristics to handle sparsity and run to solve a set of linear optimization problems in order to analyse their computational performance. 


\section{Organização do Texto}

No capítulo 1 foram feitos um breve histórico sobre otimização lincar enfocando sua evolução até os dias de hoje. a apresentação dos problemas de otimização linear canalizados e esparsos e uma pequena citação sobre o método dual simplex canalizado com busca lincar por partes especializado para resolver estes problemas.

No capítulo 2 foram apresentados um pouco da teoria básica sobre dualidade (definições, corolários e teoremas) enfocando a formulação do problema dual, as relações entre o par de problemas primal e dual, cm especial os problemas de otimização linear canalizados e. mais especificamente os problemas de otimização linear com restrições canalizadas, para o qual detalhamos o método dual simplex canalizado com busca linear por partes.

No capítulo 3, apresentamos de maneira mais detalhada: a forma produto da inversa e como atualizar a inversa da matri\% básica nas iterações subsequentes. Apresentamos também a decomposição LL; proposta por Bartels-Golub [2]. como resolver os sistemas lineares quando a atualização da decomposição I.U é feita e algumas considerações sobre implementações. Finalmente, descrevemos sobre estabilidade numérica na decomposição L.U da matriz básica e quando e com que frequência fazer uma nova refatoração da matriz.

No capitulo 4, descrevemos três diferentes métodos para atualizar a decomposição I.U da matri\% básical semelhante aos métodos do capitulo anterior. porém buscando manter a esparsidade original dos dados. () primeiro método foi proposto por Forrest-Tomlin [15] e os outros dois, chamados de variantes de Bartels-Golub [2]. foram propostos por Reid.

No capítulo 5, apresentamos duas heurísticas de pivoteamento que são utilizadas junto com a decomposiçâo LU com o objetivo de prescrvar a esparsidade existente nas matrizes básicas do método simplex. $\Lambda$ primeira heuristica descrita é a de Markowiz [26]. Tal 
heurística é simples e, apesar de muitas outras heurísticas terem sido desenvolvidas c implementadas, esta ainda é considerada bem eliciente (Duff [13]). $\Lambda$ segunda heurística é a de Grau Minimo (Vanderbei [41]). também bastante simples e possui a vantagem de ser de implementação fácil.

No capítulo 6. apresentamos a estrutura de dados utilizada nas implementações. os geradores utilizados para realizar os testes computacionais e também, os resultados obtidos com a realização de tais testes.

Finalmente, no capítulo 7 descrevemos as conclusões e apresentamos algumas sugestões para continuidade e melhoria e deste trabalho. 


\section{Capítulo 1 - Introdução}

\subsection{Histórico}

$\Lambda$ otimiłação linear consiste na minimização ou maximização de uma função linear, definida sobre um domínio descrito por equações e/ou inequações lineares, isto é:

$$
\begin{gathered}
\text { Minimizar } f(x)-c^{T} x \\
\text { Sujcito a: } A x=b \\
x \geq 0
\end{gathered}
$$

onde, $A$ é uma matriz $m \times n, \mathrm{c}$ um vetor $n \times 1$ e b um vetor $m \times 1$. A notação $x \geq 0$ quer dizer: $x_{i} \geq 0, j=1 \ldots . n$. A função linear $f: R^{n} \rightarrow R$ é chamada lunção objetivo. Lima solução que satistaça as restrições $A x=b, x \geq 0$ é dita solução factivel.

A otimização linear é, sem dúvida, o modelo de otimização mais utilizado na prática, como por exemplo, no planejamento e controle da produção, no planejamento de recursos hidricos. rotas de veículos, rotas de mensagens em uma rede de telecomunicações. cáleulo estrutural, gerenciamento financeiro e muitas outras aplicações em problemas industriais e econômicos.

Dantzig, trabalhando num projeto no departamento da Força Aérea Americana, apresentou. em 1947, uma forma sistemática de resolução dos problemas de otimização linear, o método simplex. A partir desta data, pode-se dizer que a otimização linear tornou-se realidade e um grande número de pesquisadores tem contribuído para o campo da otimização linear de diferentes maneiras, incluindo desenvolvimentos teóricos, aspectos computacionais e novas aplicaçōes.

A abordagem por otimização linear desfruta de ampla accitação devido à habilidade de modelar problemas importantes e complexos e na capacidade de seus métodos para produzir 
soluçôes em tempo razoável. Iloje em dia, problemas de otimização linear con centenas de milhares de restrições e variáveis são resolvidos e dependendo do software e computador utilizado pode-se levar apenas alguns minutos, situação muito diferente da época em que surgiram as primeiras implementações do método simplex, onde um problema, por exemplo, com 26 restrições e 71 variáveis levava em média 8 horas para ser resolvido (Bixby [6]).

O desenvolvimento de algoritmos eficientes tem sido uma constante preocupação nestes cinqüenta anos do método simplex. Os progressos na álgebra linear têm contribuído muito para este desenvolvimento. Os dois principais progressos deste periodo a serem destacados são: a introdução da decomposição LU dinâmica usando a heurística de Markowitz [57] para selecionar o pivô (veja capítulo 5) e a resolução de sistemas lineares grandes e csparsos explorando estas caracteristicas.

A questão da cficiência do método simplex sempre foi tema de pesquisa desde sua publicação. O desempenho deste método é medido $\mathrm{cm}$ termos do número de iterações e cxperimentos computacionais realizados durantc as primeiras décadas do método simplex levaram ao folclore de um desempenho polinomial nas dimensões do problema. de grau baixo e muitas vezes linear. No entanto, Klee c Minty [22] construíram um exemplo onde o método simplex efetua um número exponencial de iterações: $2^{n}-1$ para resolver um problema de otimização linear em seu pior caso. Surgiu então a questão sobre a complexidade do problema de otimização linear. Em 1978, o matemático Khachian [21] descnvolveu um algoritmo polinomial para problemas de otimização linear, porém nunca se conseguiu uma implementação computacional deste método que fosse competitiva com o método simplex.

Em 1984. Kamarkar [20] apresentou um método de pontos interiores de complexidade polinomial, afirmando ser muito mais rápido do clue o método simplex (em alguns casos, 50 vezes mais rápido), o que estimulou intensas pesquisas na área de otimização linear, com publicação de novos algoritmos e resultados. A superioridade dos métodos de pontos intcriores foi comprovada para algumas classes de problemas, mas não em geral.

O método dual simplex foi introduzido por I emke [23] $\mathrm{cm} 1954$. Intretanto, somente no final da década de 80 é que códigos bastante utilizados na época incluíram de forma rudimentar as implementações do algoritmo dual para serem usadas na resolução de problemas intciro-misto. Mas esta situação mudou. Atualmente, o algoritmo dual simplex é uma boa alternativa em códigos modernos e testes computacionais mostram que o desempenho do algoritmo dual simplex pode ser superior ao algoritmo primal simplex (Bixby [6]).

Existem algumas razões porque as implementações do algoritmo dual simplex têm se tornado tão poderosas. A mais importante é a idéia introduzida por Goldfarb-Forrest [14]. 
uma regra chamada "stcepest-edge" para selecionar a variável que deixa a base em cada iteração dual simplex. Este método requer relativamente pequeno esforço computacional extra por iteração e é muito superior aos métodos dual padrões, em que a seleção da variável que deixa a base é baseada somente na maior infactibilidade primal.

A título de curiosidade, o termo dual é um termo matemático antigo, já o termo primal foi proposto pelo pai de Dantzig. em torno de 1954. devido à necessidade de se ter uma pequena lrase para denominar "o problema original cujo dual é..." (Dantizg [11]).

É importante dizer que além dos progressos na álgebra linear e, conseqüientemente, nos algoritmos, a evolução nos softwares para a resolução de problemas de otimização linear juntamente com a evolução dos computadores também teve um papel fundamental no desenvolvimento da otimização linear. Um excmplo disto é o software C'PI.EX, que vem sendo aperfeiçoado e melhorado desde sua primeira versão, lançada em 1988. Dentre as inúmeras melhorias feitas no CPI.EX, mais em particular nos algoritmos simplex (primal e dual) e suas variações, as principais delas foram: a introdução da regra "stecpst-edge" importante para a seleção da variável que deixa a base $\mathrm{cm}$ cada iteração, a utilização da decomposição I.L! de forma dinâmica juntamente com a heurística de pivoteamento Markowit\% [57] e também a alualização desta decomposição nas itcraçôes seguintes a ela. Outros recursos foram introduzidos no CPLEX a fím de melhorá-lo, por exemplo, o "presolve", o qual remove as equações que são redundantes, as variáveis fixas, etc, e a perturbação, a qual é usada para tratar de casos degenerados. Com relação à esparsidade, foi a partir da versão 7.1 que o CPLEX passou a explorá-la totalmente. Apenas para exemplificar o progresso do CPLEX ao longo de sua existência (quase uma década e meia) com relação ao tempo de resolução, considerando que foi usado o algoritmo dual simplex, um problema com 16223 linhas, 28568 colunas e 88340 slementos não nulos demorou 1217.4 segundos na versão 1.0, 275 segundos na versão 2.2. 260.5 segundos na versào 5.0 c 22.6 segundos na versão 7.1. Fste problema foi resolvido em uma Ultra Spare de $300 \mathrm{MHz}$ (Bixby [6]).

Uma classe de problemas de otimização linear também de grande interesse prático é a classe dos problemas de otimização linear com restrições canalizadas (Arenales [1]). os quais representam vários problemas reais. Dentre eles podemos destacar problemas da programação da produção, problemas da mistura c outros que podem ser formulados por:

$$
\begin{aligned}
& \text { Minimizar } f(x)=c^{T} x \\
& \text { Sujeito a: } r \leq A x \leq s
\end{aligned}
$$


O método dual simplex canalizado com busca linear por partes (Arenales [1]) pode ser usado para resolver este tipo de problema de otimização linear e de acordo com os testes realizados por Sousa [37], este método apresentou um desempenho computacional superior ao software comercial de otimização CPI FX $4.0 \mathrm{em}$ relação ao número de iterações necessárias para resolver o problcma (2.2), com os dados extremamente densos, porćm, seu tempo computacional foi muito maior para determinados problemas. Assim, um dos objetivos deste trabalho é resolver problemas de otimização linear canalizados e esparsos tentando explorar a csparsidade da estrutura matricial através de algumas técnicas disponíveis na literatura e com isto, reduzir o tempo computacional com relação aos testes feitos para problcmas densos.

Antes de ser apresentado, no capítulo 2, o método dual simplex canalizado com busca linear por partes para resolução de problemas de otimização linear com restrições canalizadas. veremos um pouco sobre dualidade. enfocando a formulação do problema dual, as relações entre o par de problemas primal e dual e as propriedades de factibilidade e otimalidade duais. Nos capítulos 3,4 e 5. são apresentadas algumas técnicas que podem ser usadas para realizar melhorias neste método c os resultados obtidos estão no capítulo 6 . 


\section{Capítulo 2 - Dualidade}

\subsection{Introdução}

Neste capítulo serão apresentados um pouco da teoria básica sobre dualidade (definições, corolários c teoremas) enfocando a formulação do problema dual, as relações entre o par de problemas primal e dual. em especial os problemas de otimização linear canalizados e. mais especificamente, os problemas de otimização linear com restrições canalizadas, para o qual detalhamos o método dual simplex canalizado com busca linear por partes.

\subsection{Relaxação Lagrangiana e o problema dual}

Considere inicialmente o problema primal cm sua forma padrão:

$$
\begin{aligned}
& \text { Minimizar } f(x)=c^{\prime} x \\
& \text { Sujeito a }\left\{\begin{array}{l}
A x=b \\
x \geq 0
\end{array}\right.
\end{aligned}
$$

onde $A \in R^{m \times n}$.

Sejam $a$ a $i$-ćsima linha de $A$ e o conjunto $S=\left\{x \in R^{n}, A x=h, x \geq 0\right\}$ a região de factibilidade primal. A relaxação Lagrangiana consiste em relaxar algumas restrições do problema de modo a torná-lo menos restrito, mas "pagando-se um preço", o qual estará representado na lunção objetivo caso tais restriçŏes relaxadas sejam violadas. Por exemplo, suponha que as restrições $A x=b$ foram relaxadas. ( vetor $b-A x$ indicará a violação. Sejam $h_{i} \cdots a_{i} x$ a $i$-ésima componente do vetor $b \quad A x$ e $\lambda_{,}$o preço pago pela violação da $i$-ésima restrição. Una nova função objetivo irá retratar a função objetivo primal com as restrições $A x$ b relaxadas. 
Definição: A função I agrangiana é definida por:

$L(x, \hat{\lambda})-c^{T} x+\sum_{i=1}^{m} \lambda_{i}\left(h_{i}-a_{i} x\right)-c^{l} x \cdot \hat{\lambda}^{T}(b \quad A x)=\left(c^{T}-\lambda^{T} A\right) x+\hat{\lambda}^{T} b$. para todo $\lambda \in R^{m}$.

As variáveis $\lambda_{1}, \lambda_{2}, \ldots, \lambda_{m}$ são chamadas de variáveis duais.

Definição: O problema L agrangiano é definido por:

$$
\phi(\lambda):=\min _{r, n)} L(x, \lambda)
$$

$\Lambda$ função $\not(\lambda)$ é denominada função dual.

Teorema: Para toda solução primal factível $x \cdot$ temos $f(x)>\not h(\lambda)$

De acordo com teorema acima, vemos que a função dual fornece um limitante inferior à função objetivo primal a ser minimizada. Isto sugere o seguinte problema:

Determinar $\lambda \in R^{m}$ que forneça o maior limitante inferior para a função objetivo primal.

Definição: O problema dual é definido por:

$$
\begin{gathered}
\text { Maximizar } \not(\lambda) \\
\hat{\lambda} \in R^{m}
\end{gathered}
$$

Observação: Para qualquer escolha particular de $\lambda \in R^{\prime n}$. o problema Lagrangiano, o qual define a função dual, é muito simples de ser resolvido. pois as variáveis do problema Lagrangiano: $x_{1}, x_{2}, \ldots x_{n}$. estão restritas apenas à condição de não-negatividade. Assim, se um elemento de $\left(c^{l}-\lambda^{T} A\right)$ for negativo. então $\phi(\lambda) \rightarrow-\infty$.

I.evando cm considcração a observação acima, é preciso exigir que $\left(c^{T}-\lambda^{T} A\right) \geq 0$ ou $A^{r} \lambda \leq c$, pois caso contrário não temos um limitante inferior.

Dessa forma, o domínio da função $\not(\lambda)$ fica restrito por:

$$
D=\left\{\lambda \in R^{m} / \not(\lambda)>-\infty\right\}
$$

e pode ser explicitado da seguinte maneira: 


$$
D-\left\{\lambda \in R^{n} / A^{i} \lambda \leq c^{\prime}\right\}
$$

As restrições sobre as variávcis duais são chamadas restrições duais e o conjunto $D$ ć chamado de região de lactibilidade dual.

Se $\lambda \in D$, então pode-se explicitar a finçâo dual por:

$$
\phi(\lambda)=\min _{r}\left\{\left(c^{\prime \prime}-\lambda^{\prime} A\right) x+\lambda^{\prime} \mathrm{b} / \mathrm{x} \geq 0\right\}=\lambda^{\prime} \mathrm{b}
$$

Portanto, o problema dual toma a seguinte forma:

$$
\begin{aligned}
& \text { Maximizar } \not(\lambda)=b^{\gamma} \lambda \\
& \text { Sujcito a } A^{\gamma} \lambda \leq c
\end{aligned}
$$

Definição: Uma solução $\lambda \in D$ é chamada solução dual factível. Se $\lambda^{*}$ resolve o problema duad, então $\lambda^{*}$ é chamada solução ótima dual.

\subsubsection{Relações primais - duais}

A seguir estão alguns teoremas c corolários que relacionam o par de problemas primal e dual:

Teorema: Scjam $x \in \operatorname{Se} \lambda \in D$. Fntão, $f(x) \geq \phi(\lambda)$.

Corolário: Suponha que $S \neq \varnothing$. Se o problema primal não tiver solução ótima, isto é, $f(x) \rightarrow-x$, então o problema dual será infactível.

Corolário: Suponha que $D \neq \varnothing$. Se o problema dual não tiver solução ótima, isto é, $\phi(\lambda) \rightarrow \infty$, então o problema primal será infactível.

Corolário: Se um dos problemas, primal ou dual, for infactível. então o outro não terá solução ótima.

Corolário: Se um dos problemas. primal ou dual. liver solução ótima, então o outro também terá solução ótima. 
Corolário: Sejam $x$ primal factível e $\lambda$ dual factivel. Se $f(x) \quad \not(\lambda)$, então $x$ é solução ótima primal c $\lambda$ é solução ótima dual.

Os resultados anteriores são facilmente obtidos a partir da delínição do problema dual. Os resultados a seguir são provados a partir do clássico lema de Farkas (Bazaraa [4]).

Teorema (Folgas Complementares): Dado o par de problemas primal e dual. Sejam $x \mathrm{e} \lambda$ soluções fáctiveis dos problemas primal e dual respectivamente. Uma condição necessária c suficiente para que tais soluções factiveis sejam soluções ótimas é que satisfaçam a relação:

$$
\left(c^{\prime \prime}-\lambda^{\prime} A\right) x=0 .
$$

Teorema (Fundamental da dualidade): Em relação ao par de problemas primal e dual, uma e somente uma das afirmações é verdadeira:

1- $\Lambda$ mbos os problemas tem solução ótima finita e $f(x)=\phi(\lambda)$.

2- Im dos problemas tem função objetivo ilimitada e o outro problema é infactível.

3- Nenhum dos problemas possui solução factivel.

\section{Resumindo:}

$$
\begin{array}{lll}
\text { (Primal) Otimo finito } & \Leftrightarrow & \text { Ótimo finito (Dual) } \\
\text { (Primal) Ilimitado } & \Rightarrow & \text { Infactível (Dual) } \\
\text { (Primal) Infactivel } & \Rightarrow & \text { Ilimitado/Infactivel (Dual) } \\
\text { (Dual) Ilimitado } & \Rightarrow & \text { Infactivel (primal) } \\
\text { (Dual) Infactivel } & \Rightarrow & \text { Ilimitado/Infactível (Primal) }
\end{array}
$$

Definição: Uma matriz de permutação elementar é definida a partir da matriz identidade, permutando-lhe duas linhas ou colunas. Por cxcmplo, se as linhas $i$ e $j$ são permutadas, a matri» clementar é:

$$
P=\left[\begin{array}{ccccccc}
1 & & & & & \\
& \ddots & & & & \\
& & 0 & \cdots & 1 & \\
& & & \vdots & & \\
& & 1 & & 0 & & \\
& & & & & \ddots & \\
& & & & & & 1
\end{array}\right] \leftarrow \operatorname{linha} j
$$


Definição: Sejam $P_{1}, P_{2}, \ldots . P_{k}$ matrizes de permutação elementares. Definimos como matriz de permutação $P$. a matriz resultante do produto destas matrizes elementares, ou seja, $P-P_{1} P_{2} \ldots P_{k}$

Definição (Partição básica): Suponha que posto( $A)=m$. Podemos selecionar $m$ colunas lincarmente independente da matriz $A$, obtendo uma matriz. $B \in R^{m \times m}$ e seja $N \in R^{m \times(h-m)}$ a matriz formada pelas colunas restantes de $A$. Podemos representar $A$ por:

$A P=\lfloor B N]$, onde $P$ é uma matrì de permutação.

A partição na matri\%. A dada acima é chamada partição básica, a matriz $B$ é chamada matriz básica e a matriz $N$ é chamada matriz não básica. Por simplicidade de notação, omitimos a matriz $P$.

$\Lambda$ uma partição básica $A=\mid B N]$ podemos associar:

- Solução básica primal:

$$
\hat{x}_{H}=B^{-1} b . \quad \hat{x}_{i}=0
$$

- Solução básica dual:

$$
\hat{\lambda}^{\prime}=c_{B}^{\prime} B^{\prime} \text { (Multiplicador simplex). }
$$

Se $\hat{x}_{k} \geq 0$, então a solução é factivel primal. Se $c^{I}-\hat{\lambda}^{\prime} A \geq 0$ (na verdade. basta: $\left(x-\hat{\lambda}^{\prime} N \geq 0\right.$, pois $\left.c_{B}^{\prime}-\hat{\lambda}^{\prime} B=0\right)$, $\hat{\lambda}$ é factível dual.

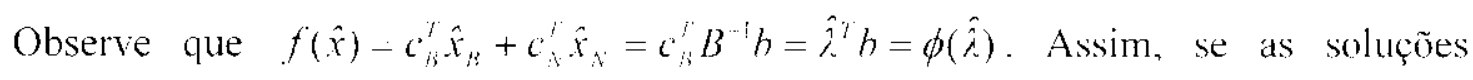
básicas forem factiveis então são soluções ótimas.

Teorema: Se um problema de otimização linear tiver solução ótima então existe uma partição básica cujas soluções básicas são ótimas.

Definição: Seja $\hat{\lambda}$ uma solução dual factivel. Se $a_{i}^{r} \hat{\lambda}=c_{i}$, dizemos que a $i$-ésima restrição dual é ativa em $\hat{\lambda}$. Caso contrário, a $i$-ésima restrição é inativa. 
Definição: Considere uma partição básica dual e $\hat{\lambda}$ a solução básica associada factível. Se

alguma restrição dual não básica for ativa em $\hat{\lambda}$, dizcmos que $\hat{\lambda}$ é dual degenerada. ou a partição básica é dual degenerada.

\subsection{Problemas de otimização linear com variáveis e restrições canalizadas}

Muitas aplicações práticas requerem variáveis cujos valores não podem exceder determinados limites. Fstão neste caso inúmeros problemas com variáveis representando niveis de produção, armazenagem ou venda e que, por razões técnicas, físicas ou comerciais, são limitadas inleriormente e superiormente, ou seja, canalizadas.

Vejamos como são as formas gerais de tais problemas:

a) Variáveis canalizadas:

Minimizar $f(x)-c^{x} x$

Sujeito a $\left\{\begin{array}{l}A x=b \\ r \leq x \leq s\end{array}\right.$

b) Restrições canalizadas:

$$
\begin{aligned}
& \text { Minimizar } f(x)-c^{r} x \\
& \text { Sujeito a } r \leq A x \leq s
\end{aligned}
$$

c) Variáveis e restrições canalizadas:

$$
\begin{aligned}
& \text { Minimizar } f(x)=c^{r} x \\
& \text { Sujeito a }\left\{\begin{array}{l}
r \leq A x \leq s \\
d \leq x \leq \mathfrak{e}
\end{array}\right.
\end{aligned}
$$

Todas as formas (2.1). (2.2) ou (2.3) são equivalentes, no sentido que qualquer problema en uma das formas pode ser escrito equivalentemente nas demais. Por simplicidade de notação, adotamos a forma (2.2) c supomos que uma matriz identidade esteja contida nas linhas da matriz, $A$. 
Antes de desenvolvermos o método dual simplex especializado para a resolução do problema (2.2), escrevemos o seu dual e mais algumas definições necessárias.

\subsubsection{O problema de otimização linear com restrições canalizadas}

Considere o seguinte problema de otimização linear com restrições canalizadas:

$$
\begin{aligned}
& \text { Minimizar } f(x)=c^{x} x \\
& \text { Sujcito a } r \leq A x \leq s
\end{aligned}
$$

onde, $A \in R^{m \times n}: s, r \in R^{n \prime} \operatorname{com} r_{l} \leq s_{i} ; c, x \in R^{n}$.

Supomos sem perda de generalidade que posto da matriz $A$ é definido por suas colunas, ou seja, posto $(A)-n$. Antes de determinarmos o problema dual do problema (2.4), consideramos a seguinte forma equivalente. Seja $y-A x$, então o problema abaixo é equivalente ao problema (2.4):

$$
\begin{aligned}
& \text { Minimizar } f(x)=c^{\prime} x \\
& \text { Sujeito a }\left\{\begin{array}{l}
A x-y=0 \\
r \leq y \leq s
\end{array}\right.
\end{aligned}
$$

\section{O problema dual:}

De maneira análoga à forma descrita anteriormente, determinamos o dual do problema (2.5). Primeiramente, definimos a função Lagrangiana:

Seja $\lambda \in R^{\prime \prime \prime}$,

$$
L(x, y, \lambda)=c^{T} x-\lambda^{\prime}(y \quad A x)=\left(c^{\prime}-\lambda^{T} A\right) x+\lambda^{7} y
$$

() problema Langrangiano é detinido por:

Para cada $\lambda \in R^{\prime \prime \prime}$, seja:

$$
\phi(\lambda)=\min _{\{x, y \mid} L(x, y, \lambda) \text { talque } r \leq y \leq s
$$

Como $x$ é irrestrito de sinal e y é canalizado, restringimos $\lambda$ tal que o mínimo em (2.7) exista. Assim temos:

$$
c^{T}-\lambda^{T} A=0 \text { ou } A^{T} \lambda=c
$$


Buscamos agora uma expressão para a função objetivo dual $\phi(\lambda)$. Lisando a restrição em (2.6) temos:

$$
L(x, y, \lambda)=\lambda_{i}^{r} y=\sum_{i=1}^{m} \lambda_{i} v_{i}
$$

Então,

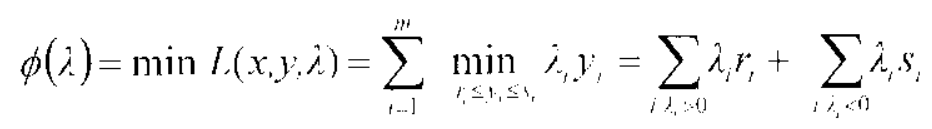

se $\lambda_{t}-0, y_{i}$ pode assumir qualquer valor no intervalo $\left|r_{i}, s_{i}\right|$.

Assim, podemos explicitar a função objetivo dual como sendo:

$$
\phi(\lambda)=\sum_{i=1}^{m} \phi_{i}\left(\lambda_{i}\right)
$$

onde,

$$
\phi_{i}\left(\lambda_{i}\right)=:\left\{\begin{array}{ll}
r_{i} \lambda_{i} & \text { se } \lambda_{i}>0 \\
s, \lambda_{i} & \text { se } \lambda_{i}<0
\end{array} .\right.
$$

Observe que o problema dual é um problema de otimização linear com a função objetivo linear por partes. Graficamente. cada parcela $\phi$, da função dual $\phi$ é representada como ilustra a figura abaixo:

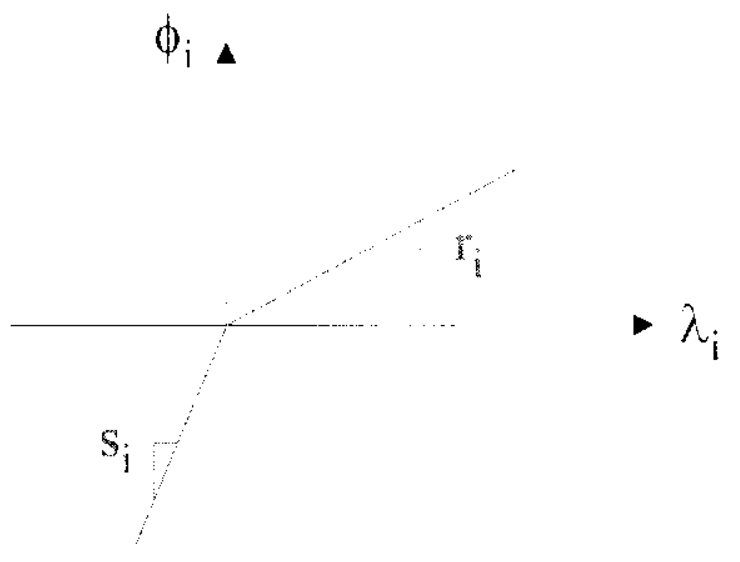

Figura 2.1 - Funçăo còncava lincar por partes 
Finalmente, obtemos o problema dual:

$$
\operatorname{Maximizar} \phi(\hat{\lambda})=\sum_{i, 1}^{m} \phi_{i}\left(\hat{\lambda}_{i}\right)
$$

Sujeito a $A^{\prime \prime} \lambda=\iota$

Observe que o dual tem $n$ restrições de igualdade e suas variáveis são irrestritas de sinal. Além disso, supomos que posto $(A)=n$. ou seja, não há equações redundantes.

F: fácil mostrar que as relações primais-duais anteriores são válidas e. em especial,

$$
f(x) \leq \phi(\lambda)
$$

para toda solução factível primal e toda solução factivel dual. Assim. se $\hat{\lambda}$ é uma solução factivel dual e $\hat{x}$ factível primal, tal que $f(\hat{x}) \leq \phi(\hat{\lambda})$, então $\hat{x}$ é ótimo primal e $\hat{\lambda}$ é ótimo dual.

\subsubsection{Soluções básicas}

Considere uma partição básica nas linhas de $A: A=\left(\begin{array}{c}B \\ N\end{array}\right)$ (ou, equivalentemente, uma partição nas colunas de $\left.A^{T}=\left(B^{T}, N^{T}\right)\right)$. Observe que esta partição define uma partição nos vetores $\hat{\lambda}, y, r$ es:

$$
A^{\prime} \lambda=c \Leftrightarrow B^{\prime} \lambda_{B}+N^{\prime} \lambda_{N}=c
$$

e

$$
\left\{\begin{array} { l } 
{ A x = y } \\
{ r \leq y \leq s }
\end{array} \Leftrightarrow \left\{\begin{array}{l}
B x=y_{H} \\
N x=y_{N} \\
r_{B} \leq y_{B}<s_{B} \\
r_{B} \leq y_{H} \leq s_{B}
\end{array}\right.\right.
$$

A esta partição associamos as seguintes soluções:

- Solução básica dual:

$$
\hat{\lambda}_{\beta}^{\gamma}=c^{\gamma} B^{-1}, \quad \hat{\lambda}_{i j}^{\gamma}=0 .
$$


- Solução básica primal:

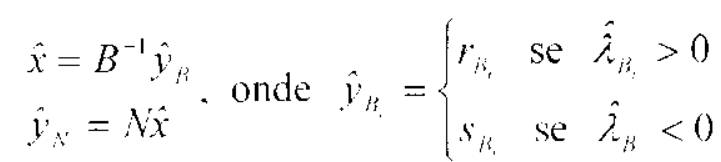

Observe que csta escolha para $\hat{y}_{k}$, resolve o problema Lagrangiano (2.9), o qual fornece a função dual. Além disso, a solução básica dual é sempre factível.

Note que:

$$
\begin{aligned}
\phi(\hat{\lambda}) & =\hat{\lambda}_{k}^{f} \hat{y}_{N}+\hat{\lambda}_{k}^{\prime} \hat{y}_{k} \quad \quad(\operatorname{veja}(2.10) \mathrm{e}(2.11)) \\
& =c^{T} B^{-1} \hat{y}_{B}=c^{T} \hat{x}=f(\hat{x})
\end{aligned}
$$

Portanto, como $\hat{\lambda}$ é factível dual, então a otimalidade segue com a factibilidade da solução básica primal:

$$
r_{N} \leq \hat{y}_{N} \leq s_{N}
$$

(já que $r_{k} \leq \hat{y}_{k} \leq s_{k}$ ).

Teorema: Considere uma partição básica $A=\left[\begin{array}{l}B \\ N\end{array}\right]$ c as soluçôes básicas associadas $(2.10) \mathrm{e}$ (2.11). Se as restrições $r_{N} \leq \hat{y}_{y_{1}} \leq s_{y}$ forem satisfeitas, então as soluções básicas primal e dual são ótimas primal e dual respectivamente.

Caso as condições do teorema não estejam satisfeitas, isto é. $r_{k}>\hat{y}_{k}$ ou $\hat{y}_{k}>s_{k}$, é possível perturbar a solução básica de modo que a função dual $\phi(\lambda)$ cresça. Para isto usamos a estratégia simplex.

\subsubsection{A estratégia simplex}

Considere uma partição básica lactível dual. A estratégia dual simplex consiste na perturbação de uma solução dual. de modo que somente uma das componentes das variáveis não básicas seja alterada, digamos a $k$-ésima variável não básica. 
De acordo com a partição da matriz $A^{T}$. podemos escrever as restrições do problema dual da seguinte forma:

$$
B^{\prime \prime} \lambda_{\nu}+N^{\prime} \lambda_{N}=\iota
$$

de onde podemos escrever a seguinte expressão para $\lambda_{t 3}$ :

$$
\lambda_{i r}-\left(B^{T}\right)^{1} c-\left(B^{T}\right)^{-1} N^{l} \lambda_{X} .
$$

$\Lambda$ solução básica ć uma solução particular fixxando-se $\hat{\lambda}_{n}=0$ e portanto, $\hat{\lambda}_{i s}=\left(B^{\gamma}\right)^{-1} c$. A estratégia simplex consiste em perturbar apenas uma componente de $\lambda_{k}$, digamos a $k$-ésima:

$$
\begin{aligned}
& \lambda_{N_{l}}=\hat{\lambda}_{H_{i}} \pm \delta, \quad \delta \geq 0 \\
& i_{i}=\hat{i}_{1} \quad j \neq k \text {. }
\end{aligned}
$$

ou seja,

$$
\lambda_{k}=\hat{\lambda}_{k} \pm \dot{x_{k}}
$$

onde $e_{k}=\left(\begin{array}{lllll}0 & \ldots & 1 & \ldots & 0\end{array}\right)$. a unidade está na $k$-ésima coordenada.

Temos dois casos para analisar:

(aso a): $\lambda_{x}=\hat{\lambda}_{k}+\dot{d e}_{k}$.

Caso b): $\dot{\lambda}_{x}=\hat{\lambda}_{x}-\dot{x}_{k}$.

Começamos pelo caso a):

$$
\lambda_{*}=\hat{\lambda}_{*}+\partial \dot{k}_{n}^{\prime}
$$

Como $\hat{\lambda}_{, 1}=0$ temos:

$$
\lambda_{Y}=\dot{x}_{k}
$$


Substituindo (2.13) em (2.12) temos:

$$
\lambda_{B}=\left(B^{T}\right)^{\prime} \mathcal{C}-\left(B^{T}\right)^{-1} N^{T} \delta e_{k} .
$$

Isando a detinição da solução básica em (2.10), podemos cscrever a equação acima da seguinte forma:

$$
\hat{\lambda}_{b^{\prime}}=\hat{\lambda}_{B^{\prime}}-\delta\left(B^{T}\right)^{-1} N^{\prime} e_{k}=\hat{\lambda}_{B}+\delta\left(-B^{i}\right)^{-1} a_{N_{i}} .
$$

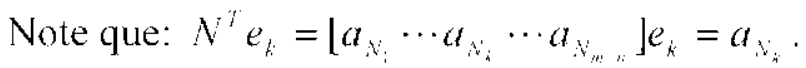

Assim,

$$
\hat{\lambda}=\left(\begin{array}{c}
\lambda_{k} \\
\lambda_{k}
\end{array}\right)=\left(\begin{array}{c}
\hat{\lambda}_{k}+\delta \eta_{k} \\
\hat{\lambda}_{N}+\dot{\delta} \dot{k}_{k}
\end{array}\right)=\hat{\lambda}_{2}+\dot{\delta} \eta
$$

onde.

$$
\eta=\left(\begin{array}{c}
\eta_{B} \\
e_{k}
\end{array}\right)=\left(\begin{array}{c}
-\left(B^{l}\right)^{-1} a_{v_{k}} \\
e_{k}
\end{array}\right) \text { é chamada de dirç̧ão simplex. }
$$

Analisando o caso b):

$$
\lambda_{k}-\hat{\lambda}_{k}-\delta \hat{e}_{k} .
$$

De acordo com (2.10) temos:

$$
\lambda_{k}=-\delta e_{k} .
$$

Substituindo (2.15) em (2.12) temos:

$$
\lambda_{B}=\left(B^{l}\right)^{\prime} c+\left(B^{r}\right)^{-1} N^{T} \delta e_{i} .
$$


Novamente de acordo com (2.10) podemos escrever a equação acima da seguinte forma:

$$
\lambda_{i s}=\hat{\lambda}_{i s}+\delta\left(B^{T}\right)^{-1} N^{\prime} e_{k}=\hat{\lambda}_{B \beta}-\delta\left(-B^{T}\right)^{-1} a_{z_{k}} .
$$

Assim

$$
\lambda=\left(\begin{array}{c}
\lambda_{\beta} \\
\lambda_{k}
\end{array}\right)-\left(\begin{array}{c}
\hat{\lambda}_{k 3}-\delta \eta_{k} \\
\hat{\lambda}_{k}-\delta \mathcal{e}_{k}
\end{array}\right)=-\dot{\lambda}-\delta \eta
$$

onde $\eta$ é a direção simplex dada por (2.14).

\subsubsection{Alteração da função dual}

Analisamos o efeito da estratégia de perturbação simplex sobre a função objetivo dual. Temos que considerar novamente dois casos:

Caso a): $\lambda=\hat{\lambda}+\delta \eta$

Caso b): $\lambda=\hat{\lambda}-\dot{\delta \eta}$.

Começando com o caso a):

A nova solução dual é:

$$
\lambda=\hat{\lambda}+\delta \eta
$$

Considerando a partição básica. $\phi\left(\lambda_{i}\right)=\sum_{i=1}^{\prime \prime \prime} \phi_{t}\left(\lambda_{i}\right)=\sum_{i=1}^{l \prime} \phi_{k_{i}}\left(\lambda_{\beta_{i}}\right)+\sum_{i=1}^{m-n} \phi_{i_{i}}\left(\lambda_{N_{i}}\right)$ podemos escrever:

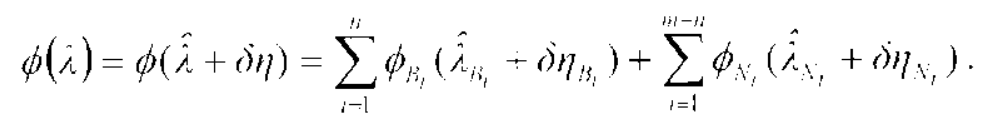

Supondo $\hat{\lambda}_{\beta_{3}} \neq 0 \quad i=1, \ldots, n$ (solução dual não degenerada) e $\delta>0$ e suficientemente pequeno,

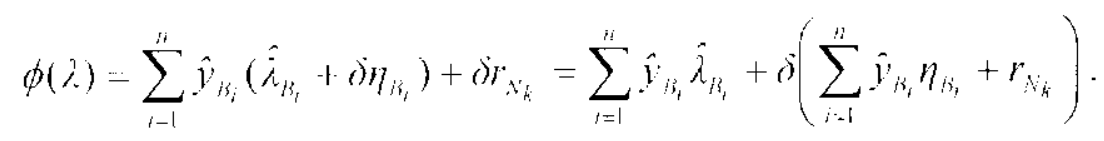


Note que:

$$
\phi_{B_{i}}\left(\lambda_{B_{1}}\right)=\hat{y}_{B_{i}} i_{B_{1}}, \quad \text { para } \hat{\lambda}_{R_{1}} \cong \hat{\lambda}_{R}
$$

e

$$
\phi_{N_{k}}\left(i_{N_{k}}\right)=r_{N_{k}} i_{N_{k}} \cdot \text { se } \lambda_{N_{i}} \geq 0
$$

Portanto,

$$
\phi(\lambda)=\phi(\hat{i})+\delta\left(-a_{x_{h}}^{Y} \hat{x}+r_{\lambda_{k}}\right)
$$

Justiticando a última equação:

I) Lembrando que $\hat{\lambda}_{N}=0$ para $i=1, \ldots, m-n$ (solução básica dual) temos:

$$
\phi(\hat{\lambda})=\sum_{i=1}^{m} \phi\left(\hat{\lambda}_{i}\right)=\sum_{i=1}^{i n} \phi_{B_{i}}\left(\hat{\lambda}_{\beta_{i}}\right)+\sum_{i=1}^{m \cdots n} \phi_{i_{i}}\left(\hat{\lambda}_{k_{i}}\right)-\sum_{i=1}^{n} \hat{y}_{k_{i}} \hat{\lambda}_{B_{i}} .
$$

II) ) Lembrando que $\eta_{B}=-\left(B^{T}\right)^{-1} a_{A_{k}}$ e $\hat{x}=B^{-1} \hat{y}_{n}$ temos:

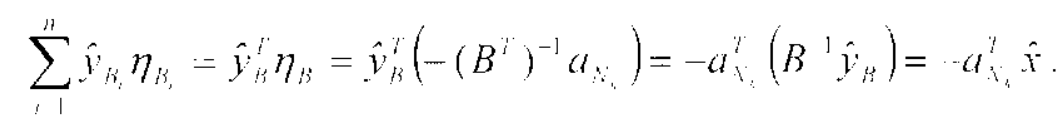

Conclusão: Como $\left(-u_{k_{k}}^{r} \hat{x}+r_{\hat{k}_{k}}\right)$ é a taxa de variação da função dual (quando $\hat{\lambda}$ é perturbado na direção $\eta$ ). então se $a_{n,}^{T} \hat{x}<r_{N_{k}}$ a estratégia simplex faz crescer a função dual. Note que a condição $a_{a}^{T}, \hat{x}<r_{n_{i}}$ significa que a $k$-ésima restrição não básica está violada.

Analisando o caso b):

A nova solução dual é:

$$
\lambda=\hat{\lambda}-\delta \eta
$$

Assim,

$$
\phi(\lambda)=\phi(\hat{\lambda}-\dot{\lambda} \eta)=\sum_{i=1}^{n} \phi_{i_{i}}\left(\hat{\lambda}_{H_{i}}-\dot{\delta} \eta_{\beta_{i}}\right)+\sum_{i=1}^{m \prime \prime} \phi_{i_{i}}\left(\hat{\lambda}_{x_{i}}-\dot{\partial} \eta_{i_{i}}\right)
$$


Supondo $\hat{\lambda}_{n} \neq 0 \quad i-1, \ldots, n$ (solução dual não degenerada) e $\delta>0$ c suficientemente pequeno, analogamente ao caso a) temos:

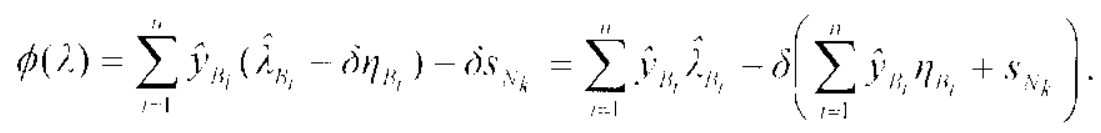

Portanto,

$$
\phi(\lambda)=\phi(\hat{i})-\delta\left(-u_{N_{h}}^{\prime} \hat{x}+s_{N_{k}}\right)
$$

As justificativas para a última equação são semelhantes ao caso a).

Conclusão: Sc $a_{v_{4}}^{\gamma} \hat{x}>s_{v_{4}}$, então a estratégia simplex faz crescer á função dual $\phi(\lambda)$, isto é. sempre que uma restrição primal é violada no limite superior, a perturbação dada pclo caso b) faz crescer a função dual.

\subsubsection{Tamanho do passo dual simplex}

Caso a): O desenvolvimento de $\phi(\hat{j}+\delta \eta \eta)$ pressupunha $\delta>0$ e suficientemente pequeno. $\mathrm{O}$ "suficientemente pequeno" significa que $\hat{\lambda}_{k_{1}}+\delta \eta_{b^{\prime}}$ não vai mudar de sinal, pois com a mudança do sinal de $\lambda_{\beta}$, o coeficiente da função objetivo é alterado, isto é, não é mais $\hat{y}_{\beta 3}$.

Se $\hat{i}_{i,}>0$ e $\eta_{\beta_{1}}>0$ não há troca de sinal.

Se $\hat{\lambda}_{\beta_{1}}>0$ e $\eta_{\beta_{1}}<0 \Rightarrow \hat{\lambda}_{B_{1}}+\delta \eta_{\beta_{1}} \geq 0 \Rightarrow \delta \leq \frac{\hat{i}_{\beta_{3}}}{\eta_{\beta_{1}}}$.

Se $\hat{i}_{\psi_{i}}<0$ e $\eta_{13_{i}}<0$ não há troca de sinal.

Se $\hat{\lambda}_{\beta_{3}}<0$ e $\eta_{\beta_{3}}>0 \Rightarrow \hat{i}_{\beta_{3}}+\delta \eta_{\beta_{i}} \leq 0 \Rightarrow \delta \leq-\frac{\hat{\lambda}_{\beta_{1}}}{\eta_{\beta_{1}}}$.

Seja $p_{f}$ um indice tal que:

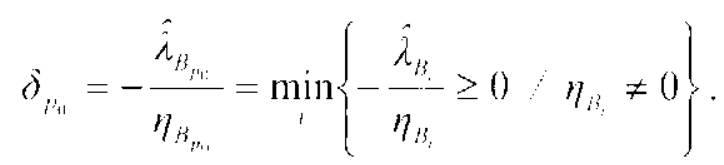


Caso b): Queremos determinar o tamanho de $\delta$ tal que $\hat{\lambda}_{\beta_{3}}-\delta \eta_{\beta_{i}}$ não mude de sinal.

Se $\hat{\lambda}_{i,}>0$ e $\eta_{i_{i}}<0$ não há troca de sinal.

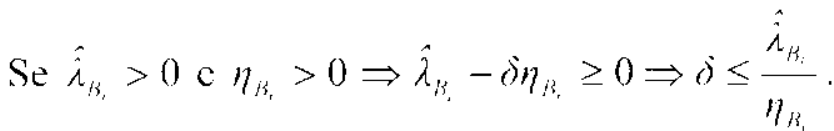

Se $\hat{\lambda}_{i<}<0$ e $\eta_{\beta_{3}}>0$ não há troca de sinal.

Se $\hat{\lambda}_{\beta_{s}}<0$ e $\eta_{\beta_{s}}<0 \Rightarrow \hat{\lambda}_{\beta_{3}}-\delta \eta_{\beta_{s}} \leq 0 \Rightarrow \delta \leq \frac{\hat{\lambda}_{B_{3}}}{\eta_{\beta_{s}}}$.

Seja $p_{\text {( }}$ um indice tal que:

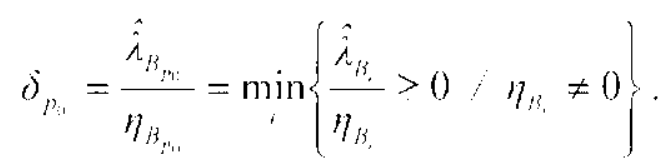

\subsubsection{Incrementando o passo dual simplex: $\delta>\delta$}

Caso a): Seja $\delta=\delta, \varepsilon \quad \varepsilon>0$ e suficientemente pequeno, isto $\dot{c}$, as variáveis básicas não trocam de sinal.

A nova solução dual é:

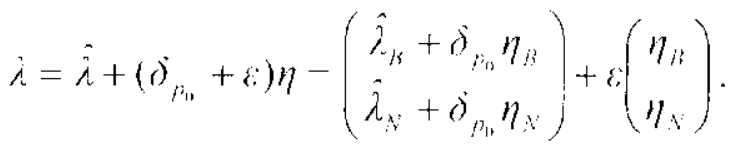

$\Lambda \operatorname{ssim}$

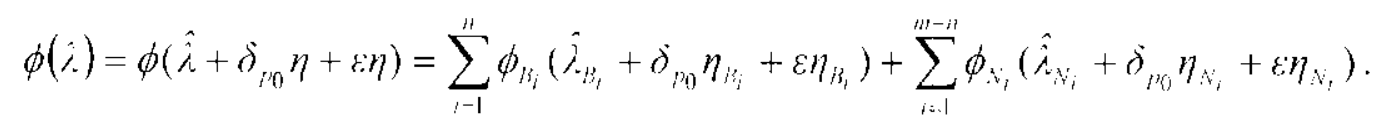

Hipótese de não degeneração: $\hat{\lambda}_{\beta_{1}}+\delta_{p_{1}} \eta_{k,}+0 . \quad i \neq \eta_{i}$.

Isto significa que o mínimo em (2.15) ocorre apenas para po, caso contrário, mais do que uma variável se anularia simultaneamente. Mas. se ocorrer múltiplas variáveis que se anulam simultaneamente a análise é similar. 
Graficamente estamos na seguinte situação:

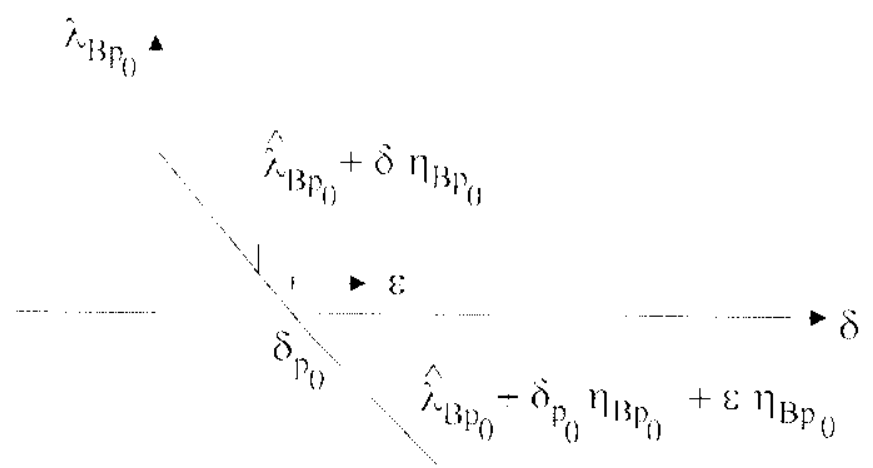

Figura 2.2 - Incremento do passo dual simplex: $\dot{j}=\delta_{p_{1}}+c$

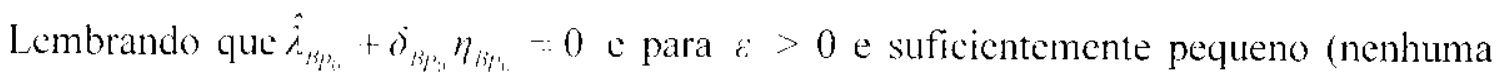
outra variável básica muda de sinal. além de $i_{i r_{1+1}}$ ):

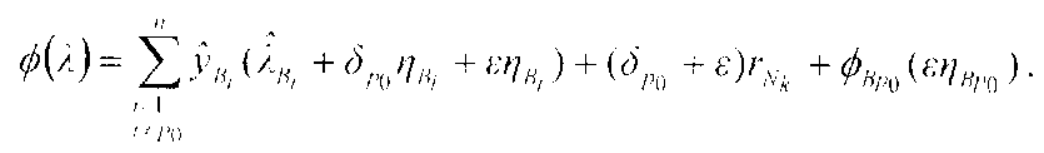

Más.

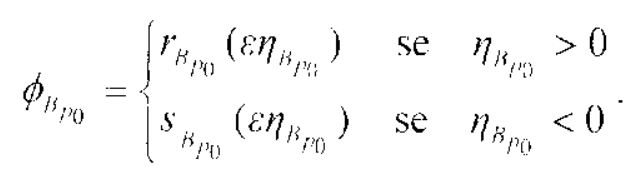

Dois casos devem ser analisados:

Casu i): $\eta_{B_{n:}}>0$

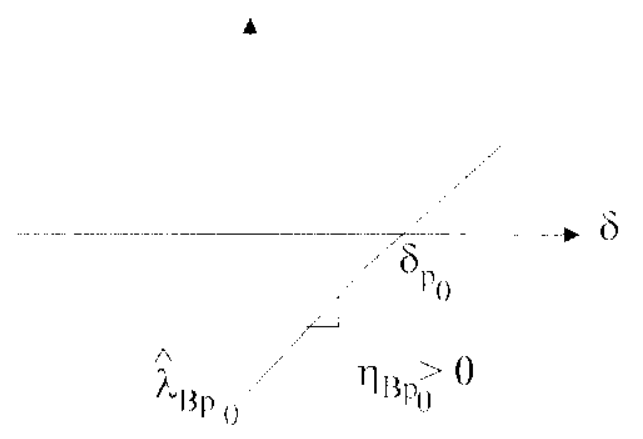

Figura $2.3-$ Caso ell clue $\eta_{i i_{i, 1}}>0$ 
Note que neste caso $\hat{\lambda}_{\beta_{p, n}}<0$ e, portanto, $\hat{y}_{k p_{1}}=s_{i / p_{1,}}$.

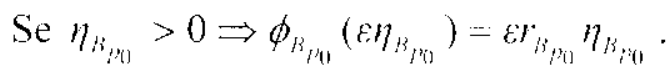

Assim. a função dual pode ser escrita da seguinte forma:

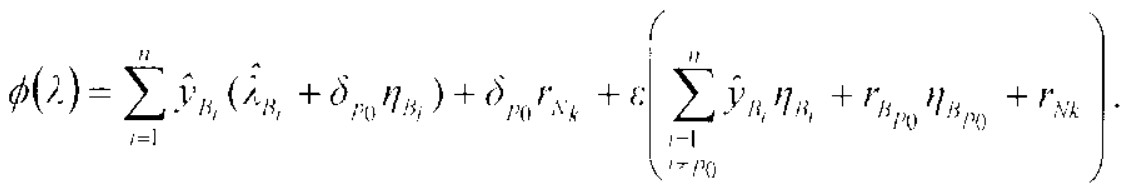

$$
\begin{aligned}
& \phi(\lambda)=\phi\left(\hat{\lambda}+\delta_{p_{1}} \eta\right)+\varepsilon\left(g_{0}-\left(s_{B_{p_{0}}}-r_{B_{p_{1}}}\right) \eta_{B_{p_{0}}}\right) .
\end{aligned}
$$

Justificando a última igualdade:

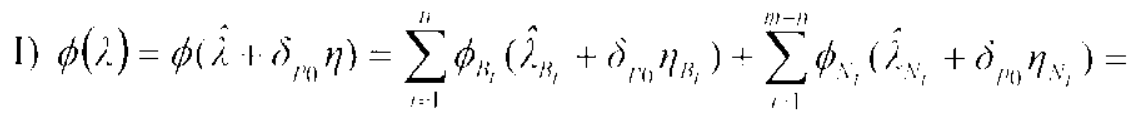

$$
=\sum_{i=1}^{n} \hat{v}_{i_{i}}\left(\hat{i}_{\beta_{i}}+\dot{\delta}_{p_{11}} \eta_{\beta_{i}}\right)+\dot{j}_{p_{i n}} r_{v k}
$$

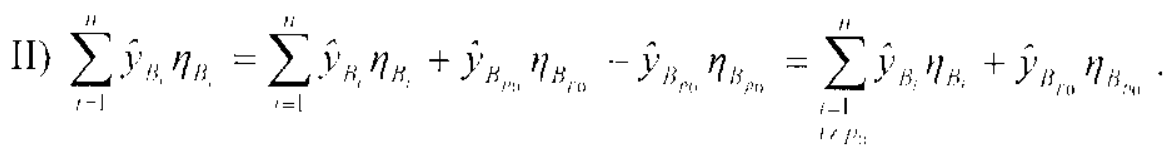

Portanto.

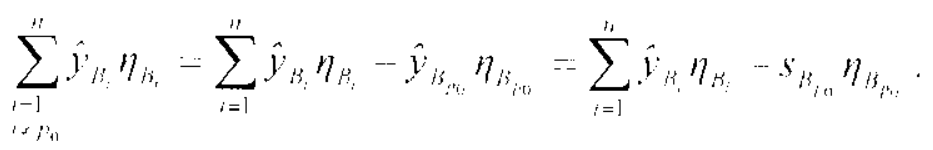

III) Lembrando que $\eta_{n}=-\left(B^{T}\right)^{-1} a_{N_{k}}$ temos:

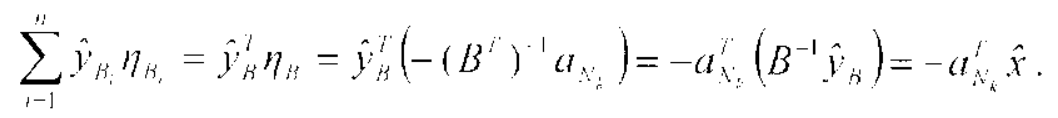

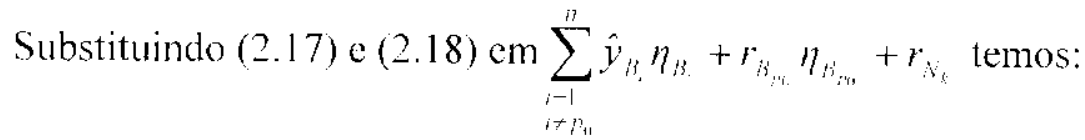




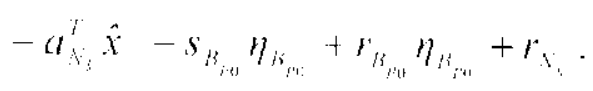

IV) Denominamos $g_{0}=\cdots a_{N_{i}}^{T} \hat{x}+r_{r_{i}}$.

Observe que a função dual que antes tinha a taxa de variação $g_{0}=a_{\hat{k}_{4}}^{\gamma} \hat{x}+r_{\mathrm{s}_{\mathrm{a}}}>0$ agora :

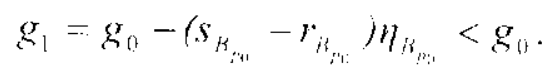

Caso ii): $\eta_{B_{i+1}}<0$.

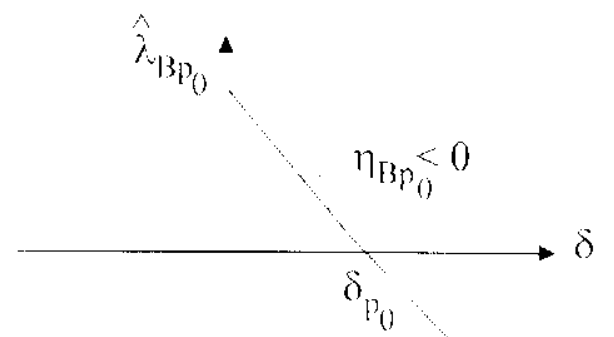

Figura 2.4 - Caso em que $\eta_{n_{n: 1}}<0$

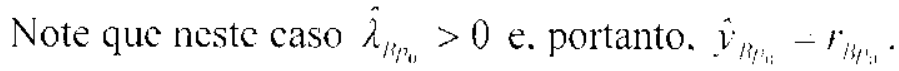

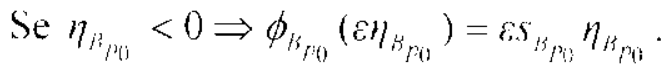

Assim. a função dual pode ser escrita da seguinte forma:

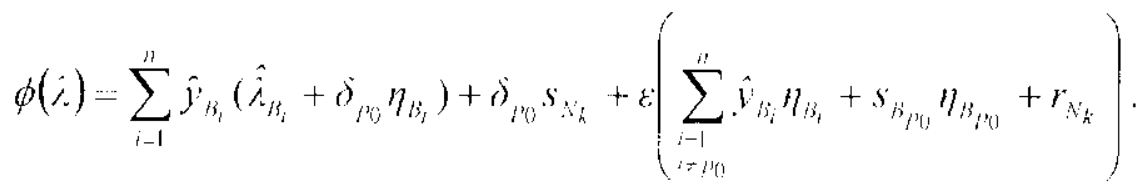

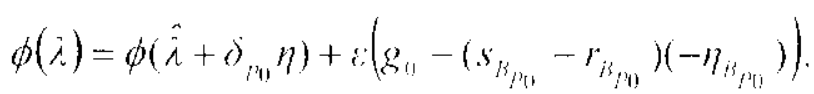

Justificando a última igualdade: 


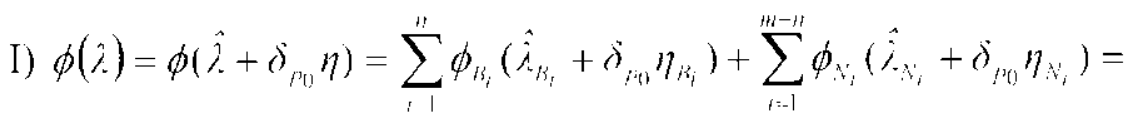
$=\sum_{i=1}^{n} \hat{y}_{B_{i}}\left(\hat{\lambda}_{k_{i}}+\delta_{p_{t 1}} \eta_{k_{i}}\right)+\delta_{n_{i}} s_{k_{i}}$

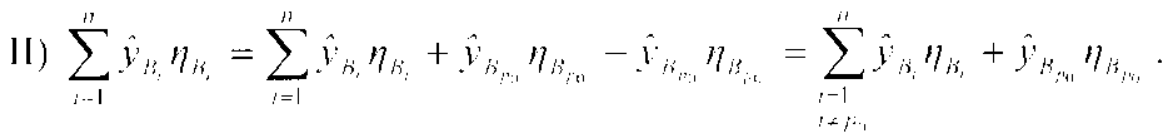

Portanto,

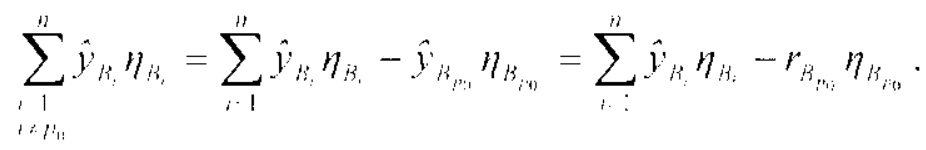

III) L cmbrando que $\eta_{\beta}=-\left(B^{\gamma}\right)^{\prime} a_{\beta_{i}}$ temos:

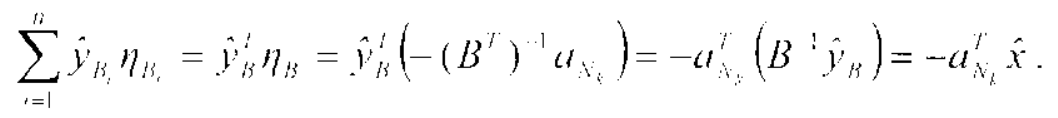

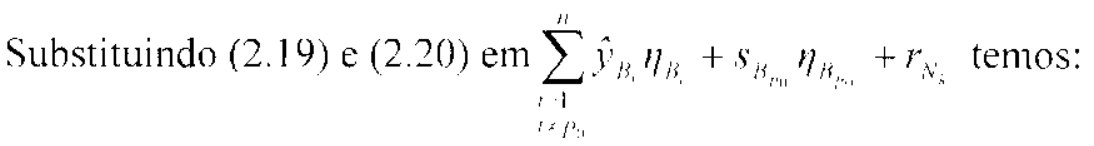

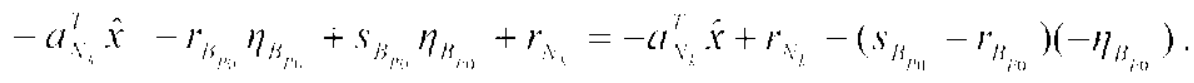

IV) Denominamos $g_{0}=-a_{Y,}^{T} \hat{x}+r_{i}$.

Finalmente, obtemos a seguinte expressão para a função dual:

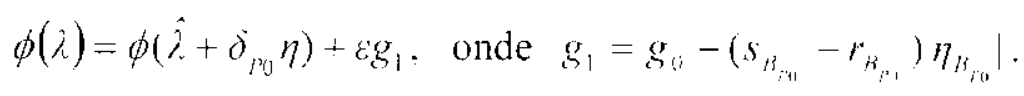

Caso b): Seja $\delta=\left(\delta_{n:}+\varepsilon\right), \quad \delta>0$ e suficientemente pequeno.

A nova solução dual ć:

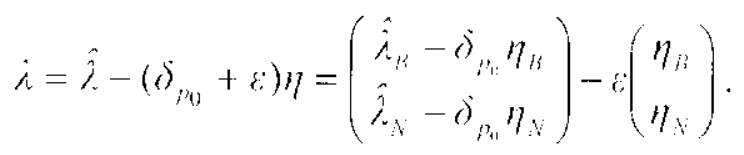


Analisando de mancira análoga ao caso a) e respectivos casos i) e ii), obtemos a seguinte expressão para a função dual:

$$
\phi(\lambda)=\phi\left(\dot{\lambda}-\dot{\delta}_{k_{j}} \eta\right)+\varepsilon g_{1}
$$

onde.

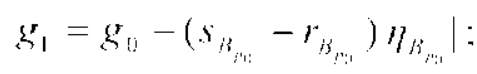

$$
\begin{aligned}
& g_{0}=a_{N_{k}} \hat{x}-s_{N_{i}} .
\end{aligned}
$$

\subsubsection{Tamanho do incremento do passo dual simplex}

As expressões da função dual na seção anterior são válidas desde que $\quad:>0$ e suficientemente pequeno. isto é, desde que as variáveis básicas não mudem de sinal. Analisemos agora qual o maior valor para o incremento $\varepsilon$.

(aso a): Determinar o tamanho do passo de modo que $\hat{i}_{\beta_{1}}+\delta_{n_{1}} \eta_{\beta_{1}}+\varepsilon \eta_{H_{1}}$ não mude de sinal.

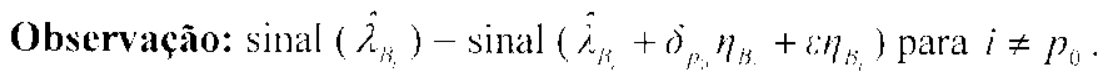

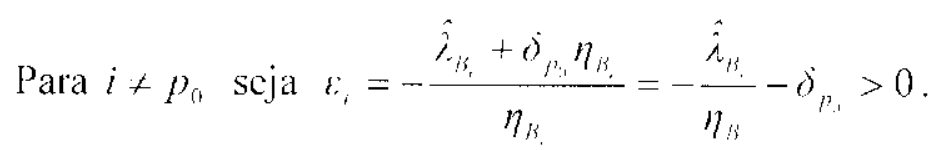

( $\delta$, é o valor de $\varepsilon$ que anula a variável básica $i_{j,}$ c, portanto, $\varepsilon>\varepsilon$, promove uma troca de sinal em $\left.\lambda_{\beta_{i}}\right)$.

Denominamos $\delta_{i}=\varepsilon_{i}+\delta_{p}=\frac{\hat{i}_{\beta_{3}}}{\eta_{\xi_{3}}}$.

( $\delta_{i}$ é o valor de $\delta$ que anula a variável básica $\left.i_{j}\right)$.

Assim, para que não haja mudança de sinal nas variáveis básicas (exceto $i_{h_{p_{1}}}$ ). o valor i) é dado por:

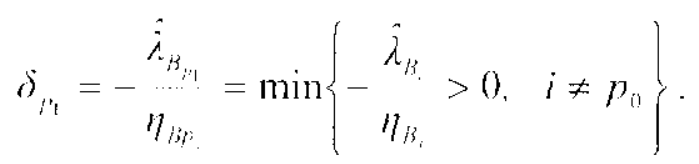


Observe que, no cálculo do índice $p_{0}$ as razões $-\frac{\hat{i}_{B_{i}}}{\eta_{B_{i}}}$ haviam sido calculadas e $p_{0}$ foi o indice da menor razão. $\Lambda$ gora $p_{l}$ é o índice da segunda menor razão.

Caso b): Determinar o tamanho do passo de modo que $\hat{i}_{i \beta_{1}}-\delta_{p_{i,}} \eta_{k_{1}}-\varepsilon \eta_{i_{i}}$ não mude de sinal.

Observação: $\operatorname{sinal}\left(\hat{i}_{\beta_{1}}\right)=\operatorname{sinal}\left(\hat{i}_{\beta_{1}}-\hat{\partial}_{i_{1}} \eta_{k_{1}}-\delta \eta_{k_{1}}\right)$ para $i \neq p_{i_{1}}$

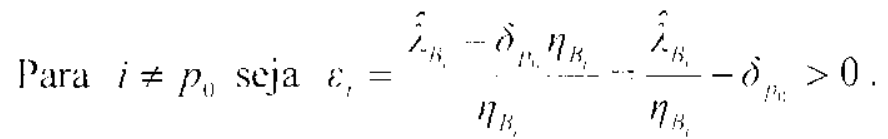

Análogo ao caso a) denominamos $\delta_{1}=\varepsilon_{i}+\delta_{p_{3}}=\frac{\hat{\hat{\lambda}}_{\beta_{1}}}{\eta_{\beta_{3}}}$ (valor de $\delta$ que promove mudança de sinal em $\lambda_{B_{3}}$ ).

Assim,

$$
\dot{\delta}_{p_{1}}=\frac{\hat{i}_{\beta_{\beta_{1}}}}{\eta_{B_{\beta_{1}}}}=\min \left\{\frac{\hat{i}_{B_{1}}}{\eta_{B_{1}}}>0, \quad i \neq p_{0}\right\}
$$

Os comentários do caso a) são análogos.

A ligura abaixo ilustra a função $\phi$ dual variando com $\delta$ :

$\phi \boldsymbol{\Delta}$

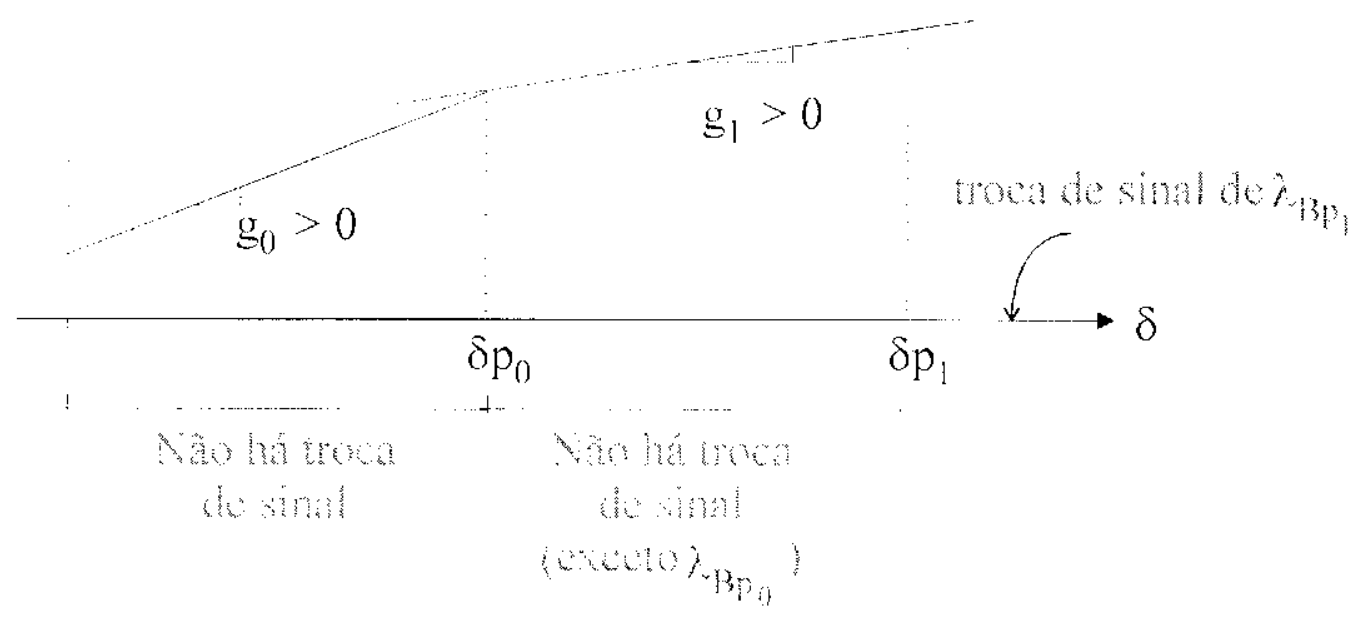

Figura 2.5 - (irático da função dual lincar por partes variando com $\delta$ 
Podemos examinar o passo dual além de $\delta_{n}: \delta>\delta_{n_{1}}$ de maneira análoga ao desenvolvimento nesta seção.

Em geral temos:

$$
\phi(\hat{i}+\dot{\partial} \eta)=\phi\left(\hat{i}+\dot{m}_{m} \eta\right)+\varepsilon g_{i+1}
$$

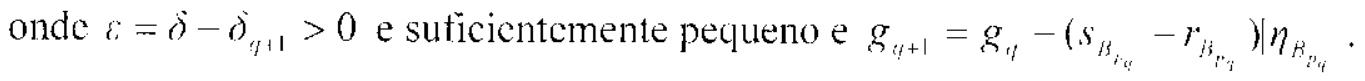

$\phi 4$

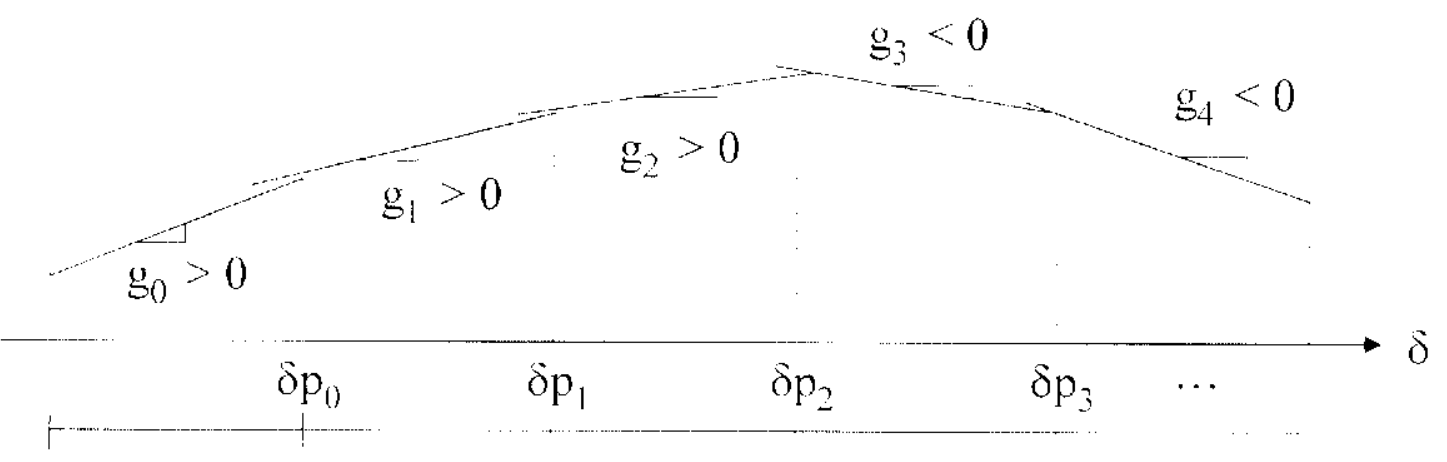

Figura 2.6 - Gráfico da função dual linear por partes no caso geral

\subsubsection{Busca linear por partes:}

Com o desenvolvimento na seção anterior é possivel escolher $\delta$ tal que: max $\phi(\hat{\lambda}+\delta \eta)$, isto é. determinar o passo que maximize a função dual na direção simplex.

A busca unidimensional consiste em escolher tal que:

$$
g_{>}>0 \quad \text { e } \quad g_{,-1} \leq 0 .
$$




\subsection{Método dual simplex canalizado com busca linear por partes}

O método dual simplex descnvolvido na sç̧ão 2.3 ć agora resumido.

\section{Fase I:}

Determine uma partição básica $A=\left[\begin{array}{l}B \\ N\end{array}\right]$ nas linhas de $A$ (usualmente $B=I$. Caso as variáveis não scjam canalizadas, pode-se, sem perda de generalidade, impor-se limites suficientemente grandes para as variáveis).

$$
\mathrm{P} \Lambda \mathrm{RE}=\mathrm{F} \Lambda \mathrm{ISO} \text {. }
$$

$t-0$.

\section{Fase II:}

Enquanto NÃO PARE, faça:

\{Cálculo da solução dual\}

$$
\hat{\lambda}=\left[\begin{array}{c}
\hat{i}_{k} \\
\hat{i}_{-N}
\end{array}\right]=\left[\begin{array}{cc}
\left(B^{1}\right)^{T} & c \\
0
\end{array}\right]
$$

\{Cálculo da solução primal\}

$$
\begin{aligned}
& \hat{x}=B^{-1} \hat{y}_{k} \\
& \hat{y}_{3}=N \hat{x}
\end{aligned} \quad \text { onde } \quad \hat{y}_{i,}=\left\{\begin{array}{lll}
r_{k} & \text { se } & \hat{\lambda}_{\beta_{3}} \geq 0 \\
s_{k,} & \text { se } & \hat{\lambda}_{\beta_{3}} \leq 0
\end{array} .\right.
$$

Observação: Caso $\hat{i}_{\beta_{2}}=0$, isto é, solução degenerada. a escolha de $\hat{v}_{k}$ é arbitrária inicialmente. convencionando-se que se $\hat{y}_{k_{1}}=r_{k_{1}}$, então $\hat{\lambda}_{k_{1}}$ é considerado positivo para cfeito do cálculo do passo.

\{Verificaçăo de otimalidade\}

Se $r_{i} \leq \hat{y}_{N} \leq s_{N}$. então PARE - VERDADE (a solução atual é ótima).

Senão.

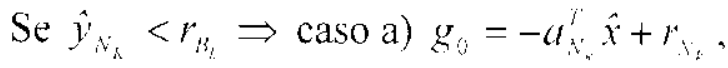

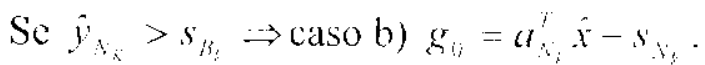


Determine a dircção dual-simplex:

$$
\eta=\left(\begin{array}{l}
\eta_{k} \\
\eta_{k}
\end{array}\right)=\left(\begin{array}{c}
-\left(B^{\prime}\right){ }^{\prime} a_{k_{k}} \\
e_{k}
\end{array}\right)
$$

\{Determinação do passo\}

Determine os $\delta_{i}^{*}$ s tal que:

$$
\begin{aligned}
& \text { (aso a): } \delta_{i}=\left\{-\frac{\hat{i}_{B_{i}}}{\eta_{B_{i}}} \geq 0\right\} . \\
& \text { (aso b): } \delta_{i}=\left\{\frac{\hat{i}_{B_{i}}}{\eta_{B_{i}}} \geq 0\right\} .
\end{aligned}
$$

Observação: $\mathrm{Sc} \hat{\lambda}_{p_{i}}=0$. caso degencrado, $\mathrm{c} \hat{y}_{H_{t}}=r_{i_{i}}$ então $j$, é calculado somente se $\eta_{b_{1}}>0$.

Ordene os $\delta_{i}$ s de forma a obter:

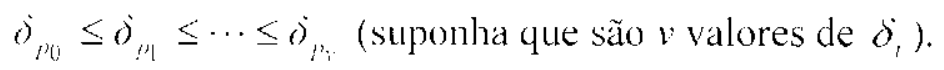

\{Busca unidimensional\}

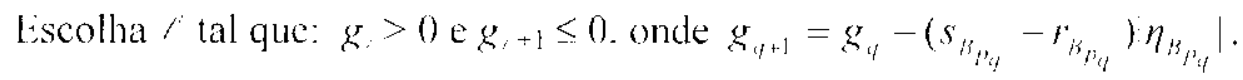

Se $g_{n, 1}>0$ então $\phi(\hat{\lambda}+\dot{\partial} \eta) \rightarrow \infty$ co problema dual não tem solução ótima co o primal é infactível.

$$
\text { PARE }=\text { VIERD } \triangle D E .
$$

\{Atualização\}

\{Troque o $k$-ésimo índice básico pelo t-ésimo índice não básico\}

Determinar a nova partição básica:

$$
\begin{aligned}
& B_{t} \leftrightarrow N_{p}, \\
& t \leftarrow 1-1 .
\end{aligned}
$$




\section{Exemplo 2.1:}

Considere o seguinte problema de otimização linear:

- Problema Primal:

$$
\begin{aligned}
& \text { Minimizar } f(x)-\cdots 5 x_{1}-2 x_{2} \\
& \text { Sujeito a }\left\{\begin{array}{c}
-1 \leq-x_{1}+x_{2} \leq 3 \\
-2 \leq x_{2}+x_{2} \leq 8 \\
-1 \leq x_{1} \leq 6 \\
0 \leq x_{2} \leq 4
\end{array}\right.
\end{aligned}
$$

- Problema Dual:

$$
\begin{aligned}
& \text { Maximizar } \phi(\lambda)=\sum_{i=1}^{1} \phi_{1}\left(\lambda_{i}\right) \\
& \text { Sujeito a }\left\{\begin{array}{r}
-\lambda_{1}+\lambda_{i}+\lambda_{i}=-5 \\
\lambda_{1}+\lambda_{2}+\lambda_{4}=-2
\end{array}\right.
\end{aligned}
$$

onde,

$$
\begin{aligned}
& \phi_{1}\left(\lambda_{1}\right)=\left\{\begin{array}{ccc}
-1 \lambda_{1} & \text { se } & \lambda_{1}>0 \\
3 \lambda_{1} & \text { se } & \lambda_{1}<0
\end{array} .\right. \\
& \phi_{2}\left(\lambda_{2}\right)=\left\{\begin{array}{ccc}
-2 i_{2} & \text { se } & \lambda_{2}>0 \\
8 i_{2} & \text { se } & \lambda_{2}<0
\end{array}\right. \text {. } \\
& \phi_{i}\left(\lambda_{3}\right)=\left\{\begin{array}{rrr}
-1 i_{3} & \text { se } & \lambda_{3}>0 \\
6 i_{3} & \text { se } & i_{3}<0
\end{array}\right. \text {. } \\
& \phi_{.:}\left(\lambda_{4}\right)=\left\{\begin{array}{ccc}
0 & \text { se } & \lambda_{1}>0 \\
4 \lambda_{4} & \text { se } & \lambda_{4}<0
\end{array} .\right.
\end{aligned}
$$

\section{Fase I:}

Partição básica dual factível inicial: $A=\left(\begin{array}{l}N \\ B\end{array}\right)=\left(\begin{array}{cc}-1 & 1 \\ 1 & 1 \\ \hline 1 & 0 \\ 0 & 1\end{array}\right)$.

Índices básicos: $\quad B_{1}=3 . \quad B_{2}-4$.

Índices não básicos: $\quad N_{1}=1, \quad N_{2}=2$. 


\section{Fase II:}

\section{Iteração 1:}

$$
B=\left(\begin{array}{ll}
1 & 0 \\
0 & 1
\end{array}\right), \quad N=\left(\begin{array}{cc}
-1 & 1 \\
1 & 1
\end{array}\right) .
$$

- Determinando a solução básica dual associada:

$$
\begin{aligned}
& \hat{\lambda}=\left[\begin{array}{l}
\hat{\lambda}_{k} \\
\hat{\lambda}_{N}
\end{array}\right]=\left[\begin{array}{c}
\left(B^{\prime}\right)^{-1} c \\
0
\end{array}\right]=\left(\begin{array}{c}
5 \\
-2 \\
0 \\
0
\end{array}\right) . \\
& B^{T} \hat{\lambda}_{i s}=c \text {. } \\
& \left(\begin{array}{ll}
1 & 0 \\
0 & 1
\end{array}\right)\left(\begin{array}{l}
\hat{\lambda}_{H_{1}} \\
\hat{\lambda}_{B_{2}}
\end{array}\right)=\left(\begin{array}{l}
-5 \\
-2
\end{array}\right) \text {. } \\
& \hat{i}_{/ 3}=\left(\begin{array}{l}
-5 \\
-2
\end{array}\right) \text {. }
\end{aligned}
$$

- Determinando a solução básica primal associada:

$$
\begin{aligned}
& \hat{y}_{B_{1}}=\hat{y}_{3}=6 . \\
& \hat{y}_{B_{2}}=\hat{y}_{1}=4 . \\
& B \hat{x}=\hat{y}_{3} . \\
& \left(\begin{array}{ll}
1 & 0 \\
0 & 1
\end{array}\right) \cdot\left(\begin{array}{l}
\hat{x}_{1} \\
\hat{x}_{2}
\end{array}\right)=\left(\begin{array}{l}
6 \\
4
\end{array}\right) \\
& \hat{x}=\left(\begin{array}{l}
6 \\
4
\end{array}\right) . \\
& \hat{y}_{N}=N \hat{x}=\left(\begin{array}{ll}
-1 & 1 \\
1 & 1
\end{array}\right)\left(\begin{array}{l}
6 \\
4
\end{array}\right)=\left(\begin{array}{l}
-2 \\
10
\end{array}\right)
\end{aligned}
$$

- Verificando a otimalidade:

$$
\begin{array}{ll}
N_{1}=1: & \hat{y}_{1}=-2<-1 \text { (violada) } \\
N_{2}=2: & \hat{y}_{2}=10>8 \text { (violada) }
\end{array}
$$

$\Lambda$ solução atual não é ótima. pois as duas restriçôs de canalização não estão satisfeitas. 
- Determinando $k$ :

$\Lambda$ restrição mais violada é a segunda.

Portanto: $k-2$.

$\hat{y}_{k_{2}}>s_{N^{\prime} 2}$, cntão estamos no caso b).

- Determinando a direção dual simplex:

$$
\eta-\left(\begin{array}{cc}
-\left(B^{l}\right)^{\prime} a_{v_{2}} \\
e_{2}
\end{array}\right)=\left(\begin{array}{c}
-1 \\
\cdots \\
0 \\
1
\end{array}\right) .
$$

- Determinando $\delta_{i}$ :

$$
\begin{aligned}
& \text { Caso b): } \delta_{i}=\left\{\frac{\hat{\lambda}_{B_{1}}}{\eta_{k}}>0\right\} . \\
& \delta_{0}=-5=5 . \\
& \delta_{2}=\frac{-2}{-1}=2 .
\end{aligned}
$$

- Ordenando os $\delta_{1}^{\prime}$ 's temos:

$$
\begin{aligned}
& \delta_{p 0}=2 . \\
& \delta_{j}=5 .
\end{aligned}
$$

- Busca linear por partes:

$$
\begin{aligned}
& g_{0}=a_{v_{2}} \hat{x}-s_{k_{2}}=\left(\begin{array}{ll}
1 & 1
\end{array}\right) \cdot\left(\begin{array}{l}
6 \\
4
\end{array}\right)-8=2>0 \text { (violação original). } \\
& g_{1}=g_{0}-\left(s_{\beta_{r, 1}}-r_{\beta_{i, 1}}\right) \eta_{\beta_{i+1}}|=2-(4-0)|-1 \mid=-2<0 .
\end{aligned}
$$

Portanto,

$$
\ell=0 \ell N_{p}=N_{2} \text {. }
$$


- Alualizando:

$$
B_{2} \leftrightarrow N_{2}
$$

\section{Iteração 2:}

Indices básicos: $\quad B_{1}-3, \quad B_{2}=2$.

Índices não básicos: $\quad N_{1}-1, \quad N_{2}-4$.

$$
B=\left(\begin{array}{ll}
1 & 0 \\
1 & 1
\end{array}\right) . \quad N=\left(\begin{array}{cc}
-1 & 1 \\
0 & 1
\end{array}\right) .
$$

- Determinando a solução básica dual associada:

$$
\begin{aligned}
& \dot{\lambda}=\left[\begin{array}{l}
\hat{\lambda}_{B} \\
\hat{\lambda}_{A}
\end{array}\right]=\left[\begin{array}{cc}
\left(B^{T}\right)^{\prime} c \\
0
\end{array}\right]=\left(\begin{array}{c}
-3 \\
-2 \\
0 \\
0
\end{array}\right) . \\
& B^{\prime} \hat{\lambda}_{t j}=c \text {. } \\
& \left(\begin{array}{ll}
1 & 1 \\
0 & 1
\end{array}\right) \cdot\left(\begin{array}{l}
\hat{i}_{i k_{1}} \\
\hat{i}_{i k 2}
\end{array}\right)=\left(\begin{array}{l}
-5 \\
-2
\end{array}\right) \\
& \hat{i}_{j 3}=\left(\begin{array}{l}
-3 \\
-2
\end{array}\right) \text {. }
\end{aligned}
$$

- Determinando a solução básica primal associada:

$$
\begin{aligned}
& \hat{y}_{B_{1}}=\hat{y}_{3}=6 . \\
& \hat{y}_{B_{2}}=\hat{y}_{2}=8 . \\
& B \bar{x}-\hat{y}_{3} . \\
& \left(\begin{array}{ll}
1 & 0 \\
1 & 1
\end{array}\right)\left(\begin{array}{l}
\hat{x}_{1} \\
\hat{x}_{2}
\end{array}\right)=\left(\begin{array}{l}
6 \\
8
\end{array}\right) . \\
& \hat{x}=\left(\begin{array}{l}
6 \\
2
\end{array}\right) .
\end{aligned}
$$




$$
\hat{y}_{N}=N \hat{x}=\left(\begin{array}{cc}
-1 & 1 \\
0 & 1
\end{array}\right)\left(\begin{array}{l}
6 \\
2
\end{array}\right)=\left(\begin{array}{c}
-4 \\
2
\end{array}\right) .
$$

- Verificando a otimalidade:

$$
\begin{aligned}
& N_{1}=1: \quad \hat{y}_{1}=-4<2 \text { (violada). } \\
& N_{2}=4: \quad 0 \leq \hat{y}_{1}=2 \leq 4 \text { (satisfeita). }
\end{aligned}
$$

A solução atual não ć ótina, pois a primeira restrição de canalização não está satisfeita.

- Determinando $k$ :

$\Lambda$ restrição mais violada é a primeira.

Portanto: $k=1$.

$\hat{v}_{x_{1}}<r_{N_{1}}$. então estamos no caso a).

- Determinando a direção dual simplex:

$$
\eta-\left(\begin{array}{cc}
\cdots\left(B^{T}\right)^{-1} a_{B_{1}} \\
e_{1}
\end{array}\right)=\left(\begin{array}{c}
2 \\
-1 \\
1 \\
0
\end{array}\right)
$$

- Determinando $\delta_{i}$ :

$$
\begin{aligned}
& \text { Caso a): } \delta_{i}=\left\{-\frac{\hat{\lambda}_{k_{i}}}{\eta_{\beta_{1}}}>0\right\} . \\
& \dot{\delta}_{1}=-\frac{-3}{2}-\frac{3}{2} .
\end{aligned}
$$

- Ordenando os $\delta_{i}^{\prime}$ 's temos:

$$
\delta_{p: 1}=1
$$


- Busca linear por partes:

$$
\begin{aligned}
& g_{01}=-a_{u_{1}}^{T} \hat{x}+r_{v_{i}}=(1-1)\left(\begin{array}{l}
6 \\
2
\end{array}\right)+(-1)=3>0 .
\end{aligned}
$$

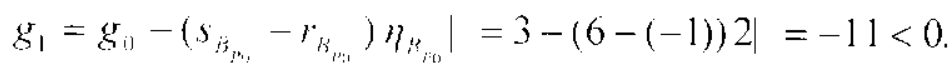

Portanto,

$$
\ell=0 \mathfrak{c} N_{l i n}=N_{1} \text {. }
$$

- Atualizando:

$$
B_{1} \leftrightarrow N_{1}
$$

\section{Iteração 3:}

Índices básicos: $\quad B_{1}-1 . \quad B_{2}=2$.

Índices não básicos: $N_{1}=3, \quad N_{2}-4$.

$$
B=\left(\begin{array}{cc}
-1 & 1 \\
1 & 1
\end{array}\right) . \quad N=\left(\begin{array}{ll}
1 & 0 \\
0 & 1
\end{array}\right)
$$

- Determinando a solução básica dual associada:

$$
\begin{aligned}
& \hat{\lambda}_{=}=\left[\begin{array}{l}
\hat{\lambda}_{B} \\
\hat{\lambda}_{x}
\end{array}\right]=\left[\begin{array}{cc}
\left(B^{\prime}\right)^{\prime} c \\
0
\end{array}\right]=\left(\begin{array}{c}
3 / 2 \\
-7 / 2 \\
0 \\
0
\end{array}\right) . \\
& B^{\gamma} \hat{\lambda}_{t h}=c \\
& \left(\begin{array}{cc}
-1 & 1 \\
1 & 1
\end{array}\right) \cdot\left(\begin{array}{l}
\hat{\lambda}_{B_{1}} \\
\hat{\lambda}_{B_{2}}
\end{array}\right)=\left(\begin{array}{l}
-5 \\
-2
\end{array}\right) . \\
& \hat{\lambda}_{B 3}=\left(\begin{array}{c}
3 / 2 \\
-7 / 2
\end{array}\right) .
\end{aligned}
$$


- Determinando a solução básica primal associada:

$$
\begin{aligned}
& \hat{y}_{B_{1}}=\hat{y}_{1}=-1 . \\
& \hat{y}_{k_{2}}=\hat{y}_{2}=8 . \\
& B \hat{x}=\hat{y}_{33} \\
& \left(\begin{array}{cc}
-1 & 1 \\
1 & 1
\end{array}\right) \cdot\left(\begin{array}{l}
\hat{x}_{1} \\
\hat{x}_{2}
\end{array}\right)-\left(\begin{array}{c}
-1 \\
8
\end{array}\right) . \\
& \hat{x}=\left(\begin{array}{l}
9 / 2 \\
7 / 2
\end{array}\right) . \\
& \hat{y}_{x}=N \hat{x}=\left(\begin{array}{ll}
1 & 0 \\
0 & 1
\end{array}\right) \cdot\left(\begin{array}{l}
9 / 2 \\
7 / 2
\end{array}\right)=\left(\begin{array}{l}
9 / 2 \\
7 / 2
\end{array}\right) .
\end{aligned}
$$

- Verificando a otimalidade:

$$
\begin{aligned}
& N_{1}=3: \quad-1 \leq \hat{y}_{3}=9 / 2 \leq 6 \text { (satisfeita). } \\
& N_{2}=4: \quad 0 \leq \hat{y}_{4}=7 / 2<4 \text { (satisfeita). }
\end{aligned}
$$

Todas as restrições estão satisfeitas. Portanto, a solução é ótima.

- Solução ótima dual:

$$
\hat{i}^{*}=\left(\begin{array}{c}
3 / 2 \\
-7 / 2 \\
0 \\
0
\end{array}\right) .
$$

- Solução ótima primal:

$$
\hat{x}_{\beta}^{*}=\left(\begin{array}{l}
7 / 2 \\
9 / 2
\end{array}\right) \text {. }
$$


- Valor ótimo da função objetivo dos problemas dual e primal:

$$
\begin{aligned}
& \phi\left(\lambda^{*}\right)=-1 .\left(\begin{array}{l}
3 \\
2
\end{array}\right)+8\left(-\frac{7}{2}\right)=-\frac{59}{2} . \\
& f\left(\hat{x}^{*}\right)=-5 \frac{9}{2}-2 \frac{7}{2}=-\frac{59}{2} .
\end{aligned}
$$

Vemos que $f\left(\hat{x}^{*}\right)=g\left(\hat{\gamma}^{*}\right)$ como era esperado.

Nas figuras abaixo podemos visualizar o caminho percorrido pelas variáveis primais e duais durante a resolução do problema.

\section{Graficamente:}

\section{- Primal:}

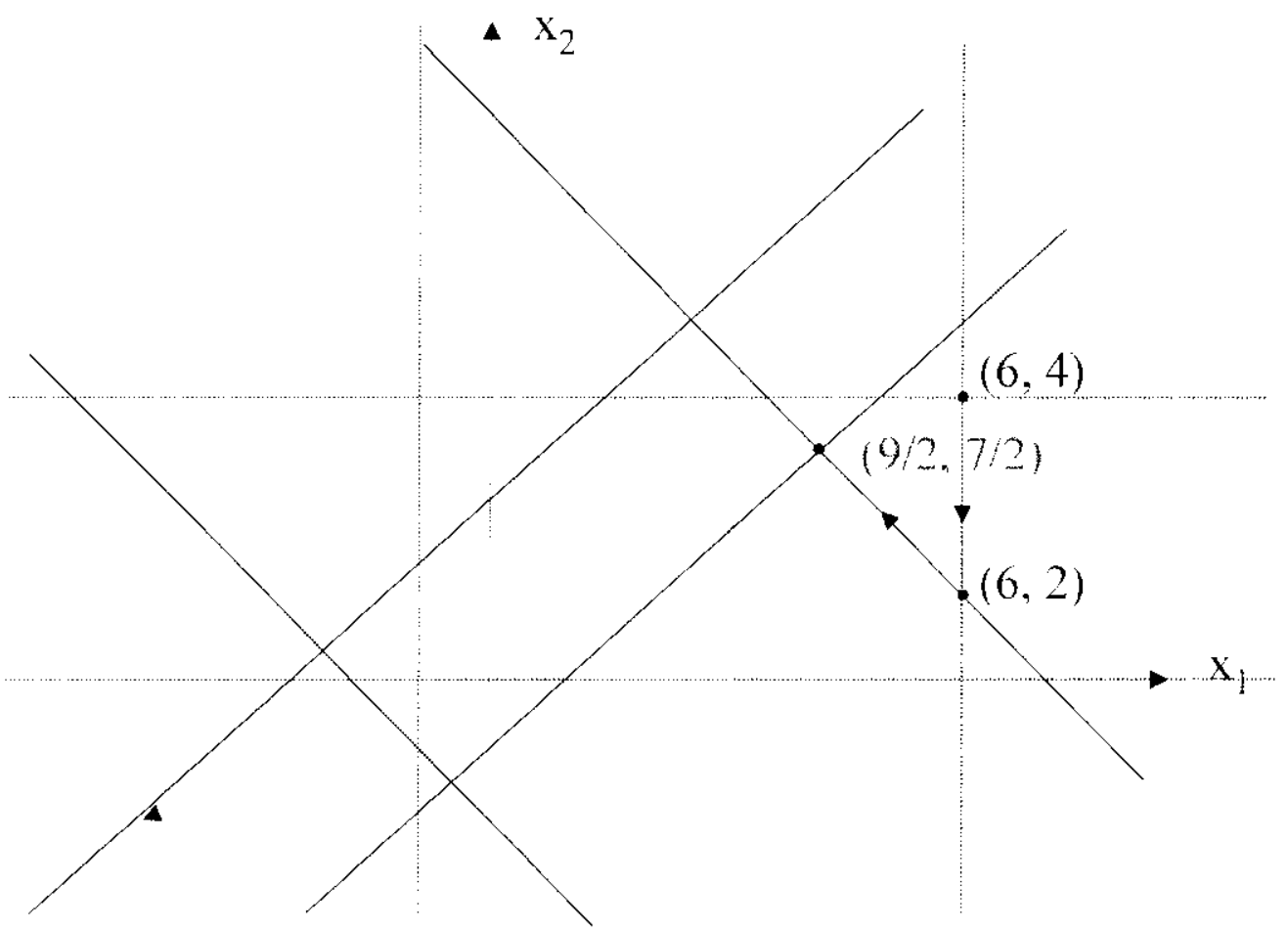

Figura 2.7 - Resolução do problema primal

No problema primal o caminho percorrido foi da solução infactivel $x^{1}=(6,4)$ para a solução também infactível $x^{2} \cdot(6,2)$ até chegar a solução factivel ótima $x^{*}=(9 / 2,7 / 2)$. 
- Dual:

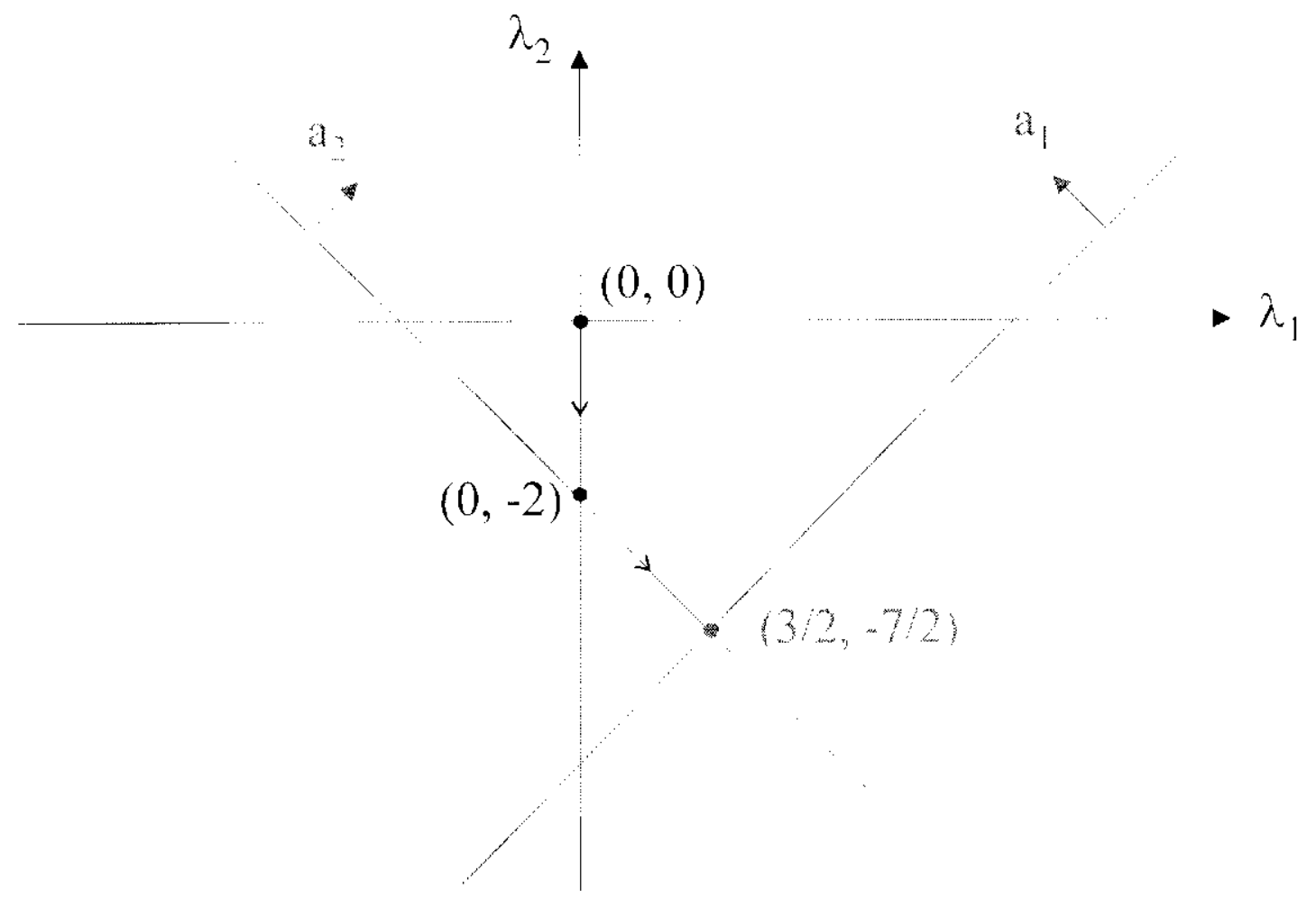

Figura 2.8 - Resolução do problema dual

No problema dual. o caminho percorrido loi da solução lactível $\lambda^{l}-(0)$, 0) para a solução também factível $\lambda^{2}=(0,-2)$ até chegar a solıção factível ótima $\lambda^{*}=(3 / 2,-7 / 2)$. 


\section{Capítulo 3 - Atualização da Base}

\subsection{Introdução}

O maior esforço computacional realizado pelo método simplex em cada iteração consiste na resolução dos seguinte's sistemas lineares:

$$
\begin{aligned}
& x_{B}=B^{-1} b \quad \Leftrightarrow B x_{\beta}=b \quad \text { (solução básica primal): } \\
& \lambda^{\prime}=c_{B s}^{\prime} B^{-1} \Leftrightarrow B^{\prime} \lambda=c_{B s} \quad \text { (vetor dos multiplicadores simplex); } \\
& d_{k}=-B^{\prime} a_{k_{k}} \Leftrightarrow B d_{\beta}=-a_{N_{k}} \quad \text { (coordenadas básicas da direção simplex). }
\end{aligned}
$$

Se estes sistemas fossem considerados isoladamente seriam necessárias $m^{3}$ operações para resolução de cada um deles. Mas os cálculos realizados para a resolução de um dos sistemas poderão ser utilizados para a resolução dos outros, pois eles têm a mesma matriz de coeficientes $B$ ou a sua transposta.

Alguns métodos têm sido utilizados para resolver tais sistemas. As primeiras implementações do método simplex trabalhavam com a inversa da matriz básica $B$ denotada por $B^{-1}$. Se tal matriz inversa era calculada explicitamente, dizia-se que estava sendo utilizada a inversa explicita, mas se a matriz inversa era armazenada como o produto de matrizes elementares, então, dizia-se que a forma produto da inversa estava sendo utilizada. IJma outra opçĩo para resolver os sistemas lineares tem sido a utilização da decomposição LU da matri\% $B$

Além da resolução dos sistemas lineares, é importante que scjam utilizados procedimentos eficientes para atualizar, de uma iteração para outra, seja a inversa ou a decomposição I.U da matriz. $B$, uma vez que apenas uma coluna de $B$ é alterada por iteração.

Neste capítulo, serão apresentadas de maneira mais detalhada: a forma produto da inversa e como atualizar a inversa da matriz básica nas iterações subsequentes é, também, a 
decomposição LU proposta por Bartels-Golub [2]. No capítulo 4 serão apresentadas outras formas de se atualizar a decomposição I.L: de uma iteração para a outra e, também, como preservar a esparsidade existente na matriz básica. um dos principais objetivos deste trabalho.

\subsection{Forma produto da inversa}

Como foi dito anteriormente. a forma produto da inversa consiste $\mathrm{cm}$ armazenar a inversa de uma certa matriz básica $B$ como o produto de matrizes elementares, ou scja, a matriz inversa $B^{-1}$ é expressa como o produto de matrizes elementares $F_{i}$. que representam as operações linhas realizadas na coluna $i$ da matriz. $B$ para torná-la uma matriz identidade.

Por exemplo: Suponha que a matriz B seja de ordem $m$. A forma produto da inversa desta matriz pode ser representada da seguinte maneira:

$$
B^{-1}=E_{m} E_{m-1} \ldots E_{1}
$$

Definịção: Uma matriz é dita elementar se ela difere da matriz identidade por apenas uma coluna, a qual representa as operações linhas realizadas em uma certa matriz.

Considere a seguinte matriz elementar:

$$
E_{i}=\left[\begin{array}{ccccc}
1 & & \alpha_{1} & & \\
& \ddots & \vdots & & \\
& & \alpha_{i} & & \\
& & \vdots & \ddots & \\
& & \alpha_{m} & & 1
\end{array}\right] .
$$

O efeito de multiplicar a matri\% elementar $E_{i}$ à esquerda de uma matriz A qualquer é:

$$
E_{1} A=\left[\begin{array}{ccccc}
1 & & \alpha_{1} & & \\
& \ddots & \vdots & \\
& & \alpha_{1} & \\
& \vdots & \ddots & \\
& \alpha_{m} & & 1
\end{array}\right]\left[\begin{array}{c}
a^{\prime} \\
\vdots \\
a^{\prime} \\
\vdots \\
a^{m}
\end{array}\right]=\left[\begin{array}{c}
a^{\prime}+\alpha_{1} a^{\prime} \\
\vdots \\
\alpha_{1} a^{\prime} \\
\vdots \\
a^{m}+\alpha_{m} a^{\prime \prime}
\end{array}\right] .
$$

ou scja, este produto corresponde as seguintes opcrações elementares:

Para $j \neq i$. multiplicar a linha $i$ por $\alpha_{j}$ e adicionar à linha $j$;

Para $j=i$, multiplicar a linha $i$ por $\alpha_{i}$. 
Os valores $\alpha_{j}, j=1, \ldots, m$. são adequadamente escolhidos de modo a transformar a coluna $i$ da matriz $\Lambda$ na $i$-ésima coluna da matriz identidade.

Neste trabalho as matrizes elementares utilizadas possuem a seguinte forma:

$$
E_{i}=\left[\begin{array}{ccccc}
1 & & & & \\
& \ddots & & & \\
& & \alpha_{i} & & \\
& & \vdots & \ddots & \\
& & \alpha_{m} & & 1
\end{array}\right] .
$$

As matrizes elementares podem ser usadas para atualizar a inversa da matriz $B$ de uma iteração para a outra.

Vejamos:

Seja $B^{(n)}=\left[a_{H_{1}} \cdots a_{b_{1}} \cdots a_{k_{n}}\right]$ a matriz básica da iteração $t \mathfrak{e}\left(B^{(t)}\right)^{-1}$ a inversa da matriz $B$ representada como o produto de matrizes elementares.

A matriz básica $B^{(t \cdot 1)}$ difere da matriz $B^{(t)}$ em apenas uma coluna, pois quando a $B$, -ésima variável básica deixa a base c a $V_{k}$-ésima variável não básica entra para a base somente uma das colunas de $B^{(t)}$ se modifica, enquanto as demais permanecem como antes. Assim. é de se esperar que a inversa de $B^{(1 /)^{1)}}$ seja obtida de maneira simples a partir da inversa de $B^{(\prime)}$.

Multiplicando $\left(B^{(t)}\right)^{-1} B^{(i+1)}$ temos:

$$
\begin{aligned}
& \left(B^{(1)}\right)^{-i} B^{(i+1)}=\left[\left(B^{(i)}\right)^{-1} a_{k_{1}} \cdots\left(B^{(t)}\right)^{-1} a_{s_{k}} \cdots\left(B^{(n)}\right)^{-1} a_{i n_{i j}}\right] \\
& =\left[\begin{array}{cccccc}
1 & 0 & & y_{1} & & 0 \\
0 & 1 & & y_{2} & & 0 \\
& & \ddots & \vdots & & \\
\vdots & \vdots & \cdots & y_{1} & \cdots & \vdots \\
& & & \vdots & \ddots & \\
0 & 0 & & y_{m} & & 1
\end{array}\right]=1 .
\end{aligned}
$$

ou seja. $\left(B^{(t)}\right)^{-1} B^{(t+1)}=\bar{I}$.

Observe que $I$ difere da matriz identidade em apenas uma coluna, no caso, a $l$-ésima coluna, isto porque. $\left(B^{(f)}\right)^{-1} a_{B_{1}}=e_{i}, i=1, \ldots,-1, t+1, \ldots, m$, onde $e_{i}$ é a $i$-ésima coluna da matris identidade. 
Observe também que $y$, é (a menos do sinal) a $\ell$-ésima coordenada básica da direção simplex, que define o tamanho do passo numa itcração simplex c, portanto, $y_{1} \neq 0$.

A inversa de $\bar{I}$ está bem definida cé facilmente obtida por:

$$
(\bar{I})^{-1}=\left[\begin{array}{cccccc}
1 & 0 & -\frac{y_{1}}{y} & & 0 \\
0 & 1 & & -\frac{y_{2}}{y_{2}} & & 0 \\
& & \ddots & \vdots & & \\
\vdots & \vdots & \cdots & \frac{1}{y} & \cdots & \vdots \\
& & & \vdots & \ddots & \\
0 & 0 & & -\frac{y_{m}}{y_{1}} & & 1
\end{array}\right] .
$$

$(1)^{-1}$ é uma matriz elementar que transforma a $/$-ésima coluna de $\bar{l}$ na f-ésima coluna da matriz identidade $\left(e_{i}\right)$.

Assim,

$$
(I)^{-1}\left(B^{(f)}\right)^{-1} B^{(t+1)}-(\bar{l})^{1} \bar{I}=I \text {. }
$$

Portanto.

$$
\left(B^{(t)}\right)^{-1}=(\bar{I})^{-1}\left(B^{(f)}\right)^{-1} \text {. }
$$

Isso significa que para determinarmos a inversa de $B^{(1+1)}$ basta multiplicarmos a esquerda da matriz $\left(B^{(t)}\right)^{-1}$, que já é conhecida, por $(\bar{l})^{-1}$. Mas, multiplicar $(\bar{l})^{\prime}$ a esquerda de uma matriz é equivalente a estender para $\left(B^{(i)}\right)^{-1}$ as operações elementares necessárias para transformar o vetor $\left(y_{1} y_{2} \cdots y_{m}\right)^{7}$ en $e_{i}$.

Denotando $E^{(1+1)}=(\bar{I})^{-1}$ e, supondo que a matriz básica da iteração inicial $B^{(0)}=I$, e $E^{(1)} . E^{(2)} \ldots . E^{(t)}$ são as matrizes elementares obtidas ao atualizar as inversas das bases nas iterações 1. 2. .... t, a matriz $\left(B^{(t+1)}\right)^{-1}$ pode ser escrita da seguinte forma:

$$
\left(B^{(1+1)}\right)^{-1}=E^{(1+1)} E^{(1)} \ldots F^{(1)} \text {. }
$$


Assim, na iteração $t$, teremos:

- Solução básica primal:

$$
x_{B \beta}=\left(B^{(t-1)}\right)^{\prime} b-E^{i+1}\left(E^{\gamma} \ldots\left(E^{\prime} h\right)\right) .
$$

- Vetor multiplicador simplex:

$$
\lambda^{\prime}-c_{B}^{i}\left(B^{(j+1)}\right)^{\prime}=\left(\left(c_{B}^{i} E^{(i-1)}\right) \cdots E^{i j)}\right) E^{(i)} .
$$

- Coordenadas básicas da direção simplex:

$$
d_{B}=-\left(B^{(t-1)}\right)^{\prime} a_{k_{k}}=-E^{(i-1)}\left(E^{i} \cdots\left(L^{\prime} a_{n_{k}}\right)\right)
$$

Observação: Para armazenar uma matriz elementar $E^{t}$, ć necessário armazenar apenas $m+1$ números: além da posição $t,\left(y_{1} / y_{i}, y_{2} / y_{i}, \ldots, y_{-1} / y_{1}, 1 / y_{m}, y_{i+1} / y_{i}, \ldots, y_{m} / y_{i}\right)$, onde o $y_{1}$ é o elemento da linha $i$ na coluna $f$ da matriz. $E$.

A soluçâo básica primal, o vetor multiplicador simplex e as coordenadas básicas da direção simplex são facilmente obtidos. pois eles são uma sequência de produtos de matrizes elementares por vetores.

\subsection{Decomposição LU de Bartels-Golub}

A decomposição I.U é uma técnica muito utilizada pela maioria dos pacotes de software para otimização linear e é equivalente à estratégia de eliminação (jaussiana, procedimento útil quando se trata de sistemas lineares gerais de grande porte e esparsos. Para evitar que os erros de arredondamento se propaguem de forma descontrolada deve-se recorrer ao pivoteamento parcial, que consiste em utilizar o elemento pivô como o maior elemento em módulo da coluna (Duff [1.3]).

Veremos a seguir. uma técnica para atualizar a decomposição LU da matriz básica $B$, a qual utiliza o pivoteamento parcial, proposta por Bartels-Golub [2].

Considere o sistema linear:

$$
x_{\beta}=B^{-1} b \Leftrightarrow B x_{\beta}=b .
$$


Os demais sistemas lineares que aparecem numa iteração simplex são tratados de forma análogà.

Suponha que desejamos resolvê-lo e que a matriz básica $B$ tenha sido decomposta no produto $L U$, onde a matriz. $Y$ é triangular superior c a matriz $L$ é triangular inferior com elementos 1 na diagonal. Tal decomposição é sempre possível, pois a permutação nas linhas de $B$ é permitida (a troca de posição entre duas linhas é também uma operação elementar).

Assim.

$P B=I$. para alguma matriz de permutação $P$.

Portanto, a resolução do sistema $B x_{b}=b$ pode ser obtida resolvendo os dois sistemas triangulares:

$$
\begin{aligned}
& l y \quad P h ; \\
& U x_{B}=y
\end{aligned}
$$

Seja $B^{10}=\left[\begin{array}{cccc}a_{B_{1}} & \cdots a_{B_{1}} & \cdots & a_{B_{n}}\end{array}\right]$ a matriz básica da iteração inicial.

Por simplicidade de notação, a partir de agora, os índices básicos $\left(B_{1} . B_{2} \ldots, B_{\mathrm{m}}\right)$ e os índices não básicos $\left(N_{1}, N_{2}, \ldots, N_{m-i n}\right)$ serão representados apenas pelos números $(1,2, \ldots, m) \mathrm{e}$ $(1,2, \ldots, m-n)$ respectivamente. Assim, a matriz $B^{(0)}$ acima. passa a ser representada da seguinte forma:

$$
B^{(1)}=\left[a_{1} \cdots a_{i} \cdots a_{m}\right]
$$

Para a próxima iteração será requerida a construção de uma nova matriz básica a $B^{(1)}$, pois a f-ésima coluna de $B^{(1)}$ deixará a base e a $k$-ćsima coluna da matriz não básica $N$ entrará para a base. A nova matriz básica $B^{(\prime)}$ terá a seguinte forma:

$$
B^{(1)}=\left[a_{1} \cdots a_{i-1} a_{k} a_{i+1} \cdots a_{m i}\right] .
$$

A decomposição de $B^{(1)}$ a partir de $B^{(1)}$ é particularmente fácil e pode ser também estável.

Vcjamos: 
A matriz básica $B^{(1)}$ tem a forma:

$$
B^{i l)}=\left[a_{1} \cdots a_{i-1} a_{k} a_{i+1} \cdots a_{m}\right] .
$$

Se colocarmos a tésima coluna $a_{w_{k}}$ na última posição da matriz c movermos todas as colunas subsequentes a ela uma posição à esquerda teremos:

$$
B^{(1)}=\left[a_{1} \cdots a_{i}, a_{i, 1} \cdots a_{m} a_{k}\right] .
$$

Assim,

$$
\begin{aligned}
L^{-1} P B^{(1)} & =\left[L{ }^{1} P a_{1} \cdots L^{-1} P a_{i,} L^{-1} P a_{i, 1} \ldots I,{ }^{1} P a_{m} I^{-1} P a_{k}\right] \\
& =\left\lfloor u_{1}, u_{2}, \ldots, u_{i-1}, u_{i, 1}, \ldots, u_{m}, I^{-1} P a_{k}\right\rfloor \\
& =H^{(1)} .
\end{aligned}
$$

onde $u_{t}$ são as colunas da matriz $U$, uma vez que $I .{ }^{\prime} P B^{(6)}=U$.

A matriz $I^{1)}$ tem a forma subtriangular superior (isto é, os elementos abaixo da subdiagonal inferior são nulos) com zeros abaixo da diagonal principal nas primeiras - colunas cono mostra a figura 3.1 abaixo:

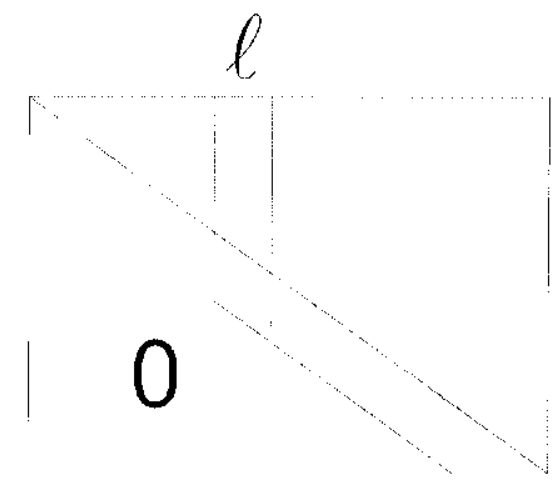

Figura 3.1 - Matriz $H^{(1)}$ subtriangular superior*.

ĺ: importante ressaltar que o vetor $l{ }^{1} P a_{k}$ já foi obtido anteriormente quando a direção simplex foi calculada, antes da atualização da base.

De fato, ao resolver o sistema $B d_{k}=-a_{k}$ temos:

$$
P B d_{n}=-P a_{k} \Rightarrow L U d_{B}=-P a_{k} \Rightarrow z=-1 \cdot{ }^{-1} P a_{k}
$$

\footnotetext{
${ }^{*} \Lambda$ matriz $/ H^{1 / 3}$ é também conhecida como matriz de llessenberg superior.
} 
Assim, a matri\% $I I^{(1)}$ pode ser construída sem qualquer esforço adicional.

$\Lambda$ matri $I^{(1)}$ pode ser redurida a uma matriz triangular superior $\left(^{(1)}\right.$ usando a eliminação Gaussiana para zerar os elementos subdiagonais nas colunas até $m$-1. A seleção do pivô é leita em cada passo da eliminação, podendo ter ou não troca entre duas linhas adjacentes. Assim, $U^{(1)}$ é obtida de $H^{(1)}$ aplicando uma sequência de operações elementares por linha em $H^{(1)}$, ou seja,

$$
U^{(1)}=E_{m-1}^{(1)} P_{m-1}^{(1)} \cdots E_{i}^{(1)} P^{(1)} H^{(1)}
$$

onde cada $F_{i}^{(1)}$ tem a forma:

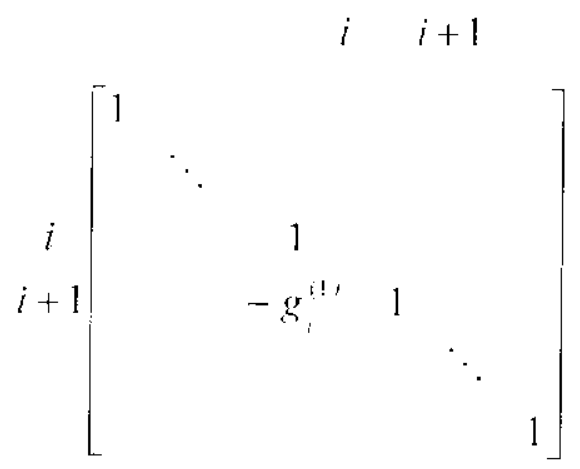

e cada $P^{(1)}$ é simplesmente a matriz identidade ou a matri identidade com a $i$-ésima e $i^{+} 1$-ésima linhas trocadas.

A escolha deve ser feita para que $\mid g_{!}^{(1) !} \leq 1$ ( $g_{1}^{(1)}$ é o multiplicador).

Substituindo (3.2) $\mathrm{cm}(3.1)$ e observando que $\left(P_{1}^{i 1 !}\right)^{-1}=P_{1}^{(1)}$ temos:

$$
P B^{(1)}=L P^{(1)} E_{i}^{(1)^{1}} \cdots P_{m !}^{(1)} E_{m 1^{(1)^{-1}}}^{(1)}
$$

Se outra iteração do Método Simplex fosse feita, a matriz básica $B^{(2)}$ resultante poderia ser decomposta a partir da decomposição de $B^{(1)}$, da mesma forma que $B^{(1)}$ foi obtida a partir da decomposição de $B^{(0)}$.

Por conveniência de notação, sejam:

$$
\begin{aligned}
& C^{(t)}=P_{i}^{(n)} E_{i}^{()^{-1}} \cdots P_{m-1}^{(n)} E_{m !}^{(n) !} \\
& G^{(t)}=C^{(1)} \cdots C^{(t)} .
\end{aligned}
$$


Supondo que a matriz básica inicial $B^{(0)}$ é a matriz identidade, então, a decomposição da matriz $B^{(t)}$ na $l$-ésima iteração é dada por:

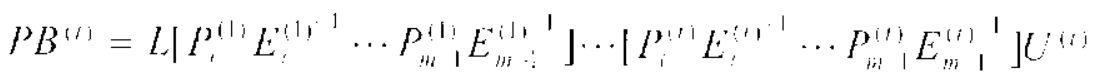

$$
\begin{aligned}
& =I C^{(1)} \cdots C^{(1)} U^{(n)} \\
& =I G^{(1)} U^{\prime \prime)} \text {. }
\end{aligned}
$$

Vejamos a seguir, um exemplo da decomposição LU de Bartels-(iolub |2].

\section{Fxemplo 3.1:}

Considere a matriz $U$ abaixo, obtida da decomposição 1 U de uma matriz básica $B^{(0)}$ qualquer.

$$
U=\left[\begin{array}{ccccc}
-1 & & & -6 & \\
& 1 & -2 & 4 & \\
& & 1 & -3 & \\
& & & 2 & \\
& & & & 1
\end{array}\right] .
$$

Vimos anteriormente, que a matriz $B^{(1)}$ é simplesmente a natriz $B^{(0)}$ com a coluna básica a, (deixou a base), substituída pela coluna não básica $a_{k}$ (entrou para a base).

Como $L^{-1} B^{(0)}-U$ e $B^{(1)}$ difere de $B^{(0)}$ em apenas uma coluna, segue que $L^{-1} B^{(1)}$ coincide com l exceto para a coluna alterada. Assim, esta coluna de $L^{-1} B^{(1)}$ é $L^{-1} a_{k}$, que já foi calculada anteriormente.

Scja a matriz $L^{-1} B^{(1)}$ :

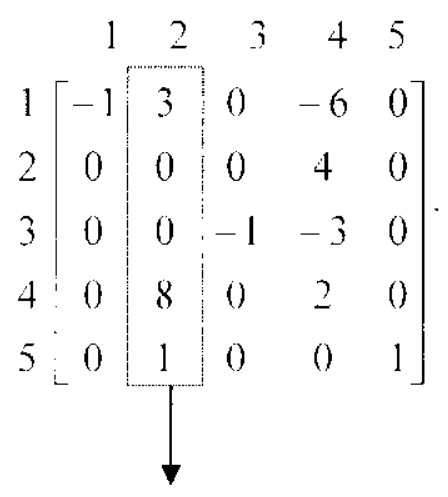

\section{Columa espeto}


Definição: É chamada de coluna espeto a coluna que contém pelo menos um elemento não nulo abaixo da diagonal.

Supondo que foi a segunda variável básica que deixou a base, a segunda coluna da matriz acima é a coluna $L^{-1} a_{2}$. Fsta é a coluna espeto.

O objetivo agora é colocar esta matriz na forma triangular superior.

Para isto, colocamos a coluna espeto na última posição da matriz e movemos as colunas 3, 4 e 5 uma posição para a esquerda. obtendo:

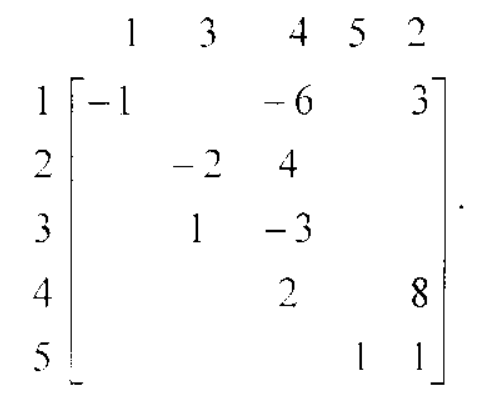

Agora. basta fazer as eliminações utilizando o pivoteamento parcial para colocar esta matriı que está na forma subtriangular superior na forma triangular superior.

- Pivô na posição $(2,2)$ :

$$
\text { pivô }=\max \{|-2|, 1\}-2 .
$$

O clemento pivô já na posição correta. Portanto, não há troca de linhas.

Zerando a posição(3, 2) da matriz temos:

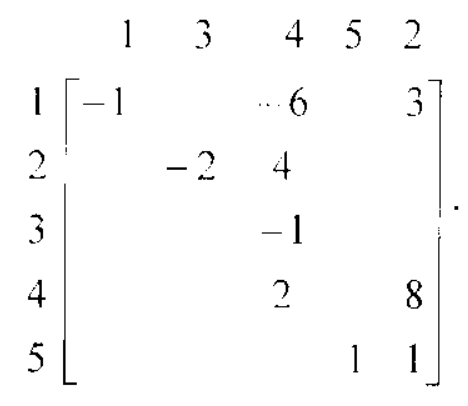

- Pivô na posição $(3,3)$ :

$$
\text { pivô }=\max \{|-1| .2\}=2 \text {. }
$$

Trocando a linha 4 com a linha 3 e zerando a posição $(4,3)$ da matriz temos: 


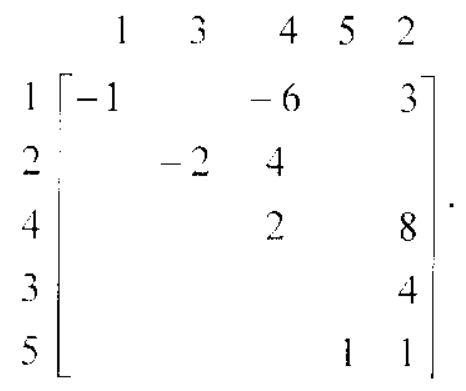

- Pivô na posição (4. 4):

Na posição do pivô existe um elemento nulo. Trocando a linha 5 com a linha 3 temos:

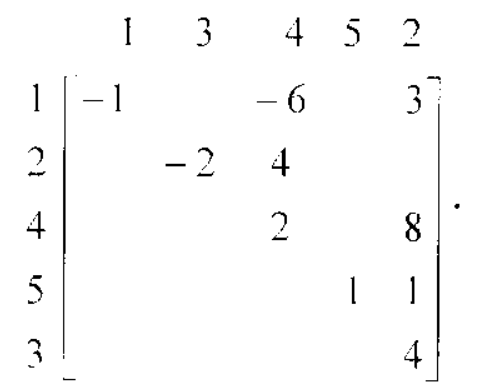

Neste caso, a climinação não é necessária, isto é, o multiplicador é nulo. Portanto:

$$
U^{(1)}\left[\begin{array}{rrrrr}
-1 & & -6 & 3 \\
& -2 & 4 & \\
& & 2 & & 8 \\
& & & 1 & 1 \\
& & & & 4
\end{array}\right]
$$

Finalmente, estamos com a matriz na forma triangular supcrior como queríamos. Se as operações elementares por linha feitas são escritas como $E_{2}^{(1)}, E_{3}^{(1)}$ e $E_{+}^{(1)}$, e as permutações de linhas são representadas por $P_{,}^{(1)} \cdot P_{3}^{(1)}$ e $P_{4}^{(1)}$. a decomposição da matriz básica $B^{(1)}$ é dada por:

$$
P B^{(1)}=L P_{2}^{(1)} E_{2}^{(1)^{-1}} P_{3}^{(1)} E_{3}^{(1)^{-1}} P_{4}^{(1)} E_{4}^{(1)-1} U^{(1)}
$$

onde. 
$E_{2}=\left[\begin{array}{ccccc}1 & & & & \\ & 1 & & & \\ & 1 / 2 & 1 & & \\ & & & 1 & \\ & & & & 1\end{array}\right], E_{3}=\left[\begin{array}{ccccc}1 & & & & \\ & 1 & & & \\ & & 1 & & \\ & -1 / 2 & 1 & \\ & & & & 1\end{array}\right]$ e $E_{1}=\left[\begin{array}{lllll}1 & & & & \\ & 1 & & & \\ & 1 & & \\ & & 1 & \\ & & & & 1\end{array}\right]$.

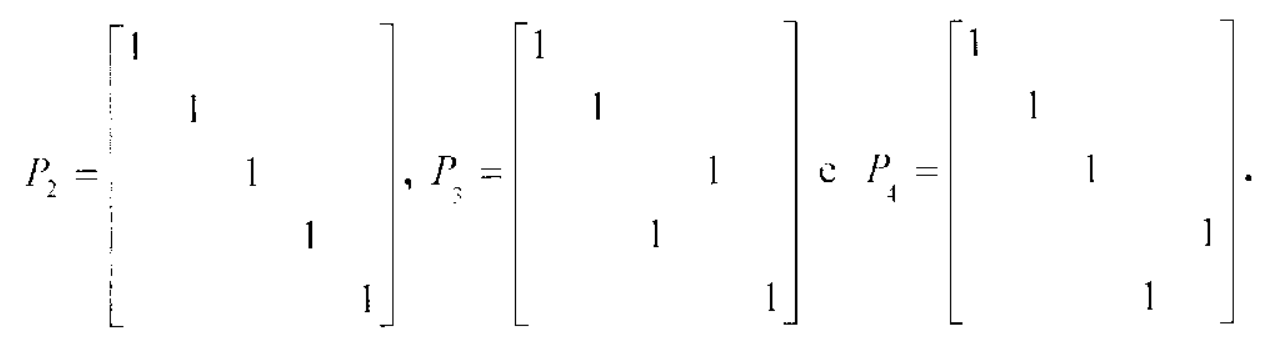

\subsection{Resolução de sistemas lineares com atualização da decomposição LU}

Feita a decomposição LU da matriz básica $B$. vejamos a resolução de sistemas lineares do tipo $B^{(i)} x_{\beta}=b$ ou $\left(B^{(t)}\right)^{t} \lambda=c_{\beta}$ na $t$-ésima iteração do Método Simplex.

1) Considere o seguinte sistema linear a ser resolvido:

$$
\begin{aligned}
& B^{(1)} x_{i s}=b . \\
& P B^{(1)} x_{b}=P b .
\end{aligned}
$$

Mas, $P B^{(1)}=L G^{(i)} H^{(t)}$. Assim, a última igualdade pode ser escrita como:

$$
L G^{(n)} U^{(n)} x_{n}=P b
$$

O próximo passo é resolver o seguinte sistema triangular inferior:

$$
L y-P h . \quad \text { onde, } \quad y=\left(i^{(i)} H^{(i)} x_{B}\right.
$$

Uma vez calculado $y$, segue que:

$$
U^{(t)} x_{\beta}=\left(\left(y^{(t)}\right)^{-1} y\right.
$$


Seja,

$$
z \cdots\left(G^{(1)}\right)^{-1} y .
$$

O vetor z é lacilmente calculado, pois

$$
z=\left\lfloor E_{m-1}^{(1)} P_{m-1}^{(n)} \cdots E_{,}^{(n)} P_{;}^{(n)}\right\rfloor \cdots\left\lfloor E_{m-1}^{(1)} P_{m-1}^{(1)} \cdots E_{,}^{(1)} P_{i}^{(1)}\right\rceil y
$$

Isto nada mais é que aplicar em y uma seqüência de operações elementares e permutações usadas nas atualizações da decomposiçào I.U da matriz básica nas iterações 1 até $t$.

rinalmente, resolvemos o seguinte sistema triangular superior:

$$
U^{(t)} x_{B}=z
$$

2) Vejamos agora como resolver o sistema linear utilizando a transposta da matriz $B^{(t)}$ :

$$
\left(B^{(H)}\right)^{T} \lambda-c_{B} .
$$

Mas,

$$
\begin{aligned}
& P B^{(1)}=L G^{(i)} U^{(I)} \\
& B^{(n)}-P^{-1} L G^{(1)} U^{(i)} .
\end{aligned}
$$

Assim, a última igualdade pode ser escrita como:

$$
\left(U^{(+1}\right)^{\prime}\left(G^{(n)}\right)^{\prime \prime}(L)^{\prime}\left(P^{-1}\right)^{\prime} \lambda=c_{i 3}
$$

() próximo passo é resolver o seguinte sistema triangular inferior:

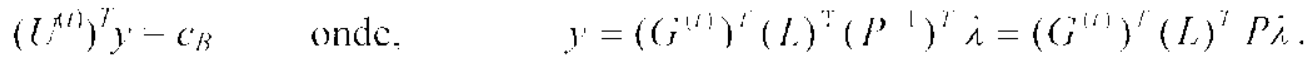

Uma vez calculado $y$. segue que:

(L.) ${ }^{T} P \lambda=\left(G^{(t)}\right)^{-T} y$ 
Seja ,

$$
z=\left(G^{(i)}\right)^{-T} y^{\prime}
$$

O vetor z é facilmente calculado. pois

$$
==\left\lceil P^{(1)}\left(E_{i}^{(1)}\right)^{-1} \cdots P_{m-1}^{(1)}\left(E_{m-1}^{(i)}\right)^{-1}|\cdots| P_{1}^{(t)}\left(E_{i}^{(i)}\right)^{-1} \cdots P_{m-1}^{(t)}\left(E_{m-1}^{(t)}\right)^{-1} \mid y\right.
$$

Isto nada mais é que aplicar em y' uma seqüência de operações elementarcs e permutações usadas nas atualizações da decomposição I U da matriz básica nas iterações laté 1 .

Seja.

$$
v=P \lambda
$$

() próximo passo é resolver o seguinte sistema triangular superior:

$$
(L)^{T} v=z
$$

Finalmente. obetmos a soluçào do sistema que é:

$$
\lambda-P^{T} v
$$

\subsection{Considerações sobre implementações}

As matrizes de permulações de linhas $(P)$ ou de colunas $(Q)$ podem ser simplesmente armazenadas como vetores de inteiros onde serão guardados apenas os índices das linhas ou colunas. Toda ver que uma linha ou coluna for permutada atualiza-se este vetor fazendo a troca dos índices que ele contém.

\section{Exemplo 3.2:}

Seja $P=\left(\begin{array}{llll}1 & 23 & 4 & 5\end{array}\right)$ o vetor que guarda os índices das linhas da matriz, $B^{(0)}$ na iteração inicial. Suponha que durante esta iteração houve apenas uma troca de linha, por exemplo, a 
linha 2 foi trocada de lugar com a linha 4 e a matriz $B^{(1)}$ foi obtida. Ao invés de fazer tal permutação nas linhas da matriz, o que acarretaria um esforço computacional maior, deve-se trocar os elementos do vetor $P$ da seguinte maneira:

$$
P-(14325)
$$

De acordo com o vetor $P$ permutado vemos que a linha 2 da matri $B^{(1)}$ corresponde à linha 4 da matriz $B^{(0)}$.

Assim, todas as permutações realizadas durante as iterações ficam representadas pelo vetor $P$.

Vimos anteriormente, que as permutações realizadas na matriz $B$ também devem ser realizadas no vetor $b$ antes de iniciar a resolução do sistema. Para isto basta re-arranjar os elementos do vetor $b$ de acordo com as permutações guardadas no vetor $P$ da seguinte forma:

$$
\begin{gathered}
\text { Para } i=1,2, \ldots . m \\
b_{i}=b_{i_{i}} .
\end{gathered}
$$

\section{Fxemplo 3.3:}

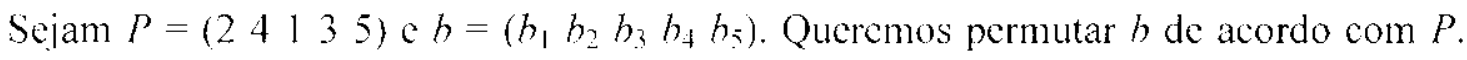
Seguindo o algoritmo acima obtemos:

$$
\begin{aligned}
& b_{1}=b_{i_{1}}=b_{2}, \\
& b_{2}=b_{i_{2}}=b_{4} . \\
& b_{3}=b_{i_{3}}=b_{1} . \\
& b_{4}=b_{1_{4}}=b_{3} . \\
& b_{5}=b_{1_{3}}=b_{5} .
\end{aligned}
$$

Portanto. o vetor $b$ permutado é o seguinte:

$$
b=\left(b_{2} b_{4} b_{1} b_{3} b_{5}\right)
$$


$\Lambda$ gora, se estamos trabalhando com a matriz transposta $B^{T}$ como na resolução do sistema lincar $B^{T} i=c_{i s}$ e temos $P^{T}$ ao invés de $P$. para rearranjar os elementos do vetor $b$ de acordo com as permutaçoes guardadas no vetor $P^{T}$ deve-se fazer o seguinte:

$$
\begin{gathered}
\text { Para } i=1,2, \ldots, m \\
b_{p_{i}}=b_{1} .
\end{gathered}
$$

\section{Exemplo 3.4:}

Sejam $P^{r}=\left(\begin{array}{llll}2 & 4 & 1 & 3\end{array} 5\right)$ e $b-\left(b_{1} b_{2} b_{3} b_{4} b_{5}\right)$. Queremos permutar $b$ de acordo com $P^{T}$. Seguindo o algoritmo acima obtemos:

$$
\begin{aligned}
& b_{r_{1}}=b_{1} \Rightarrow b_{2}=b_{i} . \\
& b_{r_{2}}=b_{1} \Rightarrow b_{4}=b_{2} . \\
& b_{i_{3}}=h \Rightarrow b_{1}=b_{3} \\
& b_{p_{4}}=b_{1} \Rightarrow b_{3}=b_{4} . \\
& b_{r_{5}}=b_{1} \Rightarrow b_{5}=b_{5} .
\end{aligned}
$$

Portanto, o vetor b permutado é o seguinte:

$$
h=\left(b_{3} b_{1} b_{4} b_{2} b_{5}\right)
$$

\subsection{Estabilidade Numérica}

Para garantir estabilidade numérica, é importante que o elemento pivô não seja muito pequeno com relação ao tamanho dos outros elementos da submatriz ativa. O pivoteamento parcial que consiste em fazer os multiplicadores em módulo menores que um, produz, em geral, uma certa cstabilidade numérica, mas quando trabalhamos com esparsidade este método não é apropriado, pois não leva em conta os preenchimentos (geração de novos elementos não-nulos). Então, para que a estabilidade numérica seja mantida. suponha que em uma dada linha $i$ da submatriz ativa, no passo t. o elemento $a_{i, /}^{\prime}$ será o pivô somente se o seguinte critério for satisfeito:

$$
\left|a_{i, j}^{\prime}\right| \geq u \cdot \max \left\{: a_{i, k}^{\prime} \mid / k \in J^{\prime}\right\}
$$


onde, $f^{t}$ ć o conjunto de indices das colunas que não foram eliminadas até o passo $t$ : ué um número real pré-fixxado tal que $0<u \leq 1$.

O crescimento dos clementos da submatriz ativa deve ser monitorado em cada passo. Assim. se a é o maior elemento $\mathrm{cm}$ módulo da matriz a ser fatorada e $d$ o maior elemento da submatriz ativa criada durante a decomposição LL: tumos:

$$
d \leq a(1+1 / u)^{m !}
$$

onde $m$ é a dimensão da matriz.

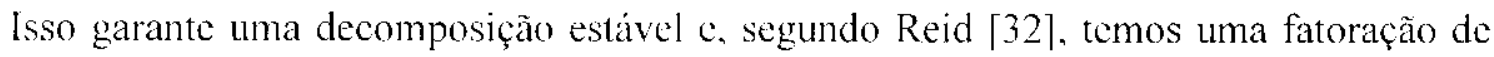
uma matriz perturbada, ou seja,

$$
L=P B Q+F
$$

Os elementos da matriz. I' satisfaz a desigualdade:

$$
\left|f_{1, i}\right| \leq(3.01) \text { d.m.g. }
$$

onde g ć a precisão da máquina que está sendo ntilizada.

Iim valor recomendado para u é 0.01 quando está sendo usada uma estratégia de pivoteamento envolvendo a tarefa de ordenar coluna, o que é uma prioridade para esparsidade. Quando a estratégia de Markowitz está em uso, um valor de $u-0.1$ é perfeitamente satisfatório (Vanderbei [41]).

Observação: Geralmente, esta técnica quando aplicada aos elementos da matriz básica $B$ com magnitudes extremamente diferentes não é muito útil. Quando isso ocorre, um reescalamento da matriz deve ser feito primeiramente.

\subsection{Refatoração da matriz básica}

Aproveitar a decomposição LU inicial da matriz básica fazendo sua atualização durante todo o processo do método simplex pode trazer alguns problemas como: acarretar um 
acúmulo nos erros de arredondamento. mesmo se empregar um critério de estabilidade, ter um esforço computacional maior com o aumento no número de matrizes elementares e ocorrer muitos preenchimentos.

Assim. uma nova decomposição L.U da matriz básica atual deve ser feita quando:

- a estabilidade numérica está sendo amcaçada. principalmente. pelo aumento no tamanho dos elementos da matriz. Portanto, o tamanho do maior elemento deve ser monitorado e uma refatoração deve ser feita se ele torna-se demasiadamente grande em módulo;

- o número de matrizes elementares $E_{i}$ aumcnta regularmente;

- o número de não nulos em $U$ também aumenta no todo.

Já sabemos quando uma nova decomposição da matri\% básica é necessária. A questão que surge agora é: Com que frequência uma nova decomposição da matriz deve ser leita? Para responder esta questão, Vanderbei [41] propôs:

Scjam,

G: o número de operações necessárias para fazer uma fatoração $L U$;

S: o número de operações para fazer a substituição progressiva c regressiva:

E: o número de operaçôes para multiplicar uma matriz por um vetor.

Assim, o número de opcraçôes para a decomposição LU da matriz básica no primeiro passo do Método Simplex é:

$$
(j+2 S
$$

Isto porque. na itcração inicial do Método Simplex é preciso lazer uma latoração $L U$ da matriz básica c duas substituições progressiva e regressiva, uma para o sistema envolvendo a matriz básica e a outra para o sistema envolvendo a transposta dela.

Para a próxima itcração são necessários duas substituições progressiva e regressiva e dois cálculos da inversa. Cada iteração subsequente é igual a anterior, exceto que existem dois cálculos extras da inversa. Portanto. o número médio de operações por iteração, se a nova decomposição for feita no passo 1 . é:

$$
\begin{aligned}
& T(t)=\frac{1}{t}((G+2 S)+2(S+E)+2(S+2 E)+\ldots+2(S+(t-1) E)) . \\
& T(t)=\frac{1}{t} G+2 S+(t-1) E .
\end{aligned}
$$


Vamos considerar $t$ como um número real por um momento. Para determinar mínimo de T. derivamos $T(t) \mathrm{em}$ relação a $t$ :

$$
\frac{d T(t)}{d k}=-\frac{1}{t^{?}} G+E
$$

Igualando a \%ero, determinamos $t$ :

$$
t=\sqrt{G i}
$$

Assim, como lé de ordem $m$ e se a matriz básica é densa, (i é de ordem $m^{3}$ e. portanto, a refatoração deve ser leita depois de $m$ iteraçôes, aproximadamente.

De fato, $l$ deve ser da ordem de $\sqrt{\frac{m^{3}}{m}}=\sqrt{m^{2}}-m$ iterações.

Agora. se a matriz básica é esparsa. ( $j$ é da ordem de $m^{2}$ o que indica que a refatoração deve ser leita depois de $\sqrt{m}$.

De fato. $t$ é da ordem de $\sqrt{\frac{m^{2}}{m}}=\sqrt{m}$.

Na prática, o valor de $/$ pode ser um parâmetro ajustável. Se não for informado usa-se $t=100$ (Vanderbei $[41 \mid)$.

No capítulo 6 realizamos alguns testes para diferentes valores de $t$. 


\section{Capítulo 4 - Atualização da Base: Esparsidade}

\subsection{Introdução}

Neste capítulo scrão apresentados três diferentes métodos para atualizar a dccomposiçẫo I.U da matriz básica semelhante aos métodos do capítulo anterior, porém buscando manter a esparsidade original dos dados. () primciro método foi proposto por Forrest-Tomlin e os outros dois. chamados de variantes de Bartels-Golub, foram propostos por Reid.

\subsection{Forma eliminação da inversa}

A idéia de que as rotinas para inversão de matrizes de otimização linear poderiam ser melhoradas em eficiência utilizando a forma eliminação da inversa ao invés da forma produto da inversa vista anteriormente no capitulo 3, foi primeira defendida por Markowitz e depois por Dantzig para matrizes especialmente estruturadas na forma "escada". Mais tarde, em experimentos com matrizes gerais de otimização linear Dantzig. Harvey, McKnight e Smith |12|. segundo Forrest-'Tomlin [15], demonstraram a superioridade da forma eliminação da inversa, a qual utiliza a decomposição $L$ L, sobre a forma produto da inversa em termos de velocidade. precisão e esparsidade.

Dadas as vantagens da forma eliminação da inversa, o próximo passo cra tentar usar os fatores triangulares resultantes. na atualização da inversa da base nas iterações seguintes. De acordo com Forrest-Tomlin [15], isso foi feito por Dantzig [10] em seu algoritmo com matrizes "escadas" de mancira a preservar a estrutura especial dos fatores triangulares e também por Bartels-Golub [2]. cujo esquema de atualização tem muitas propriedades numéricas desejáveis. 
Vários métodos para atualizar os fatores triangulares de uma matriz modificada foram propostos e após uma análise feita por lomlin em termos de suas conveniências com relação à implementação em um problema de otimização linear de grande porte. o método devido a Brayton |8]. pareceu ser o mais conveniente, principalmente em termos de implementação prática. Experimentos computacionais com este método em alguns problemas de otimização linear de tamanho médio foram muito estimulantes. A redução no crescimento de elementos não nulos na inversa foi de $50 \%$ comparada com a forma produto padrão e a redução global no tamanho do arquivo que leva à inversa da base foi de $30 \%$.

O método que veremos a seguir, produz uma matriz inversa esparsa, é uma extensão do método devido a Brayton [8/ e foi implementado por Tomlin [15].

\subsubsection{Descrição do método (Forrest-Tomlin [15])}

Primeiramente. supomos que a matriz básica $B^{(t)}$ foi decomposta no produto $L C$ utilizando a heurística de Markowitz (veja capítulo 5), com o objetivo de reduzir o número de precnchimentos, mantendo-se, assim. a esparsidade original.

Seja $B^{(h)}$ a nova matriz básica da iteração seguinte, a qual difere da anterior por apenas uma coluna. I)igamos que a coluna $f$ da base anterior foi substituída pela coluna não básica $k$ de $N$. obtendo-se $B^{(/)}$.

Considere $\alpha^{\prime}=L^{i} P a_{k}$ a forma atualizada do vetor básico $a_{k}$.

A matriz $L^{-1} P B^{113}$ não é mais triangular superior embora difira da matriz $U$ somente na coluna /. chamada coluna espeto. De acordo com o método de Bartels-Golub descrito anteriormente no capítulo 3, multiplica-se a direita de $L^{-1} P B^{(1)}$ por uma matriz de permutação $Q$. a qual coloca a coluna $\alpha^{\prime}$ na posição $m$ da matriz e move as colunas $t+1$ até m uma posição para a esquerda, ou seja,

$$
L^{\prime} P B^{(l)} Q=H
$$

A figura 4.1 abaixo mostra que a matriy. $H$ possui a forma subtriangular superior. 


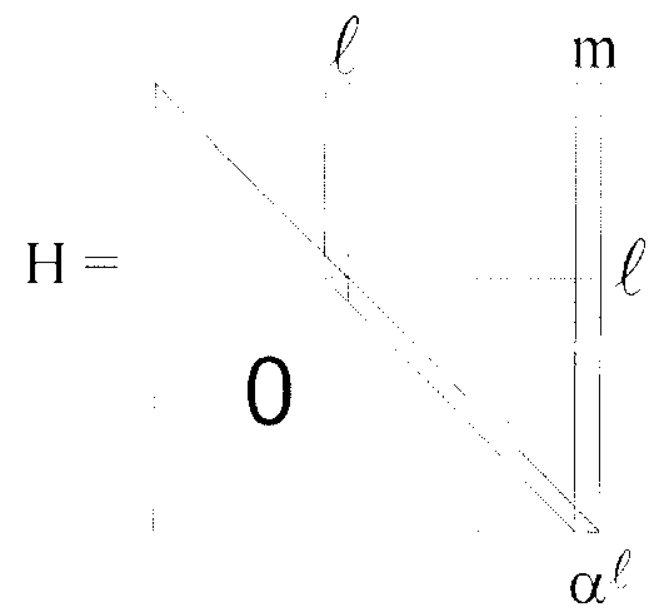

Figura 4.1- Matriz $H$ : Subtriangular superior

A matriz $/ /$ resultante pode ser colocada na forma triangular superior de várias manciras. Bartels-Golub sugerem que seja utilizado o pivoteamento parcial. Este procedimento busca controlar o crescimento dos crros de arredondamento (estabilidade numérica), mas tem a desvantagem de que. devido às trocas de linhas, preenchimentos excessivos podem ocorrer. Forrest-Tomlin [15] adotaram a estratégia de eliminar os elementos da linha $\%$, nas colunas $t$ até $m-1$ da matri subtriangular superior $H$. Para isto, deve-se multiplicar a esquerda de $H$ pela matriz $R^{-1}$, que veremos como calculá-la em seguida e então, realizar permutação de linhas para torná-la uma matriz triangular superior.

A matriz $R$ possui a seguinte forma:

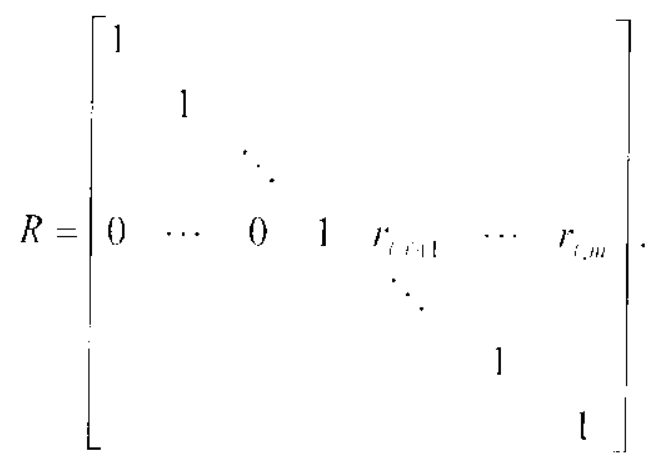

Algebricamente $R$ pode ser escrita da seguinte mancira:

$$
R-I+c_{i} r^{T}
$$

onde $e$ é um vetor de zeros com o elemento 1 somente na posição $t \mathrm{e}, r^{T}=\left(0, \ldots, r_{i} 11, \ldots, r_{m}\right)$.

A inversa da matriz. $R$ é facilmente obtida e pode ser expressa assim:

$$
R^{-1}=I-e, r^{\prime}
$$


As colunas $\ell$ até $m-1$ de $H$ são as mesmas colunas de $\ell+1$ até $m$ da matriz $U$, então a matri $R^{-1}$ elimina os elementos $h_{, \ldots}, \ldots, h_{,, \mathrm{m}-1}$ ou $u_{,, 11}, \ldots . u_{i, m}$.

listc fato pode ser usado para simplificar a determinação do vetor $r^{T}$.

Sejam.

$$
\begin{aligned}
& \widehat{u}^{\prime}=\left(0, \ldots 0, u_{i, 1,1}, \ldots u_{i, n i}\right) \\
& \text { е } \\
& r^{\prime}=\tilde{u}^{\prime} U^{-1} .
\end{aligned}
$$

Lintào,

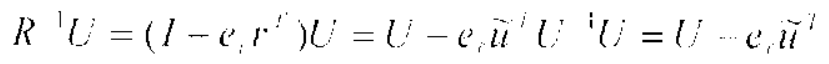

e a última expressão é simplesmente a matriz $L$ com os elementos $u_{t, 1+1}, \ldots, u_{t, m}$ sendo zeros.

Agora seja,

$$
V=R^{-1} / l
$$

Lntão. Ir difere de $/ /$ tendo os elementos $h_{i, \ldots}, \ldots, h_{i, m-1}$ substitúdos por zero e a última coluna é $R^{-} \alpha^{i}$. A matriz $V$ é claramente uma matriz triangular permutada. Se multiplicarmos a escuerda da matriz $V$ pela matriz de permutação $Q^{-1}$ (a matriz de permutação $Q$ foi usada anteriormente para colocar a matriz $U$ na forma subtriangular superior) obtemos a matriz triangular superior $U^{\prime \prime \prime}$, ou seja, $U^{(i)}-Q^{-1} V$. Para um melhor entendimento destes últimos passos, vejamos:

Seja ( ) a matriz de permutação utilizada para colocar a matriz U na forma subtriangular superior te suponha que a seguinte matriz $H$ seja o resultado do produto $Q U$ :

$$
I I=\left[\begin{array}{cccccc}
\mathrm{x} & \mathrm{x} & \mathrm{x} & \mathrm{x} & \mathrm{x} & \mathrm{x} \\
& \mathrm{x} & \mathrm{x} & \mathrm{x} & \mathrm{x} & \mathrm{x} \\
& & \mathrm{x} & \mathrm{x} & \mathrm{x} & \mathrm{x} \\
& & \mathrm{x} & \mathrm{x} & \mathrm{x} & \mathrm{x} \\
& & & \mathrm{x} & \mathrm{x} & \mathrm{x} \\
& & & & \mathrm{x} & \mathrm{x}
\end{array}\right] .
$$


Ao multiplicarmos a esquerda da matriz $/ /$ pela matriz $R^{-1}$ obtemos a seguinte matriz $V$ :

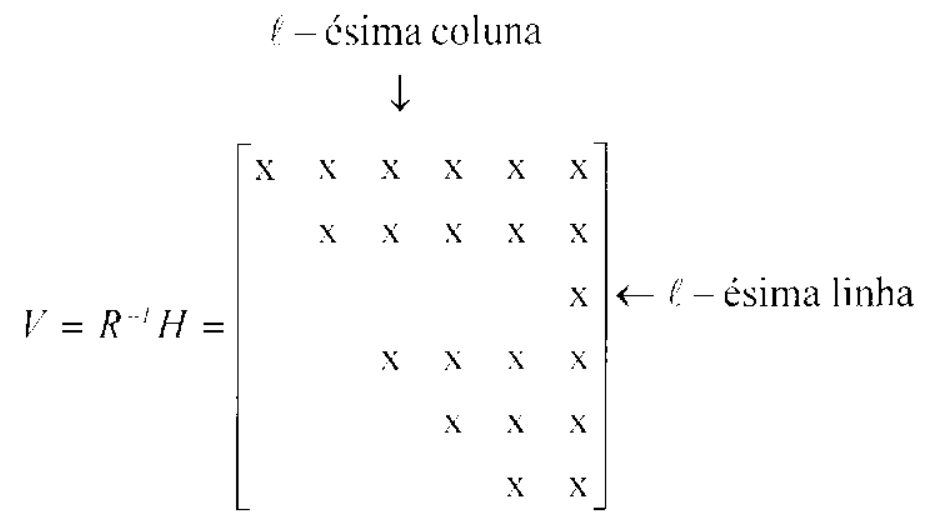

Finalmente, deve-se fazer uma permutação nas linhas da matriz $V$, para colocar a l-ésima linha na posição da $m$-ésima linha, e mover as demais linhas $"+1$ até $m$ uma posição para cima. Para isto, basta multiplicar a esquerda de $V$ pela matriz de permutação $Q^{-1}$. obtendo a seguinte matriz:

$$
U^{\prime !}=\left[\begin{array}{cccccc}
x & x & x & x & x & x \\
& x & x & x & x & x \\
& & x & x & x & x \\
& & & x & x & x \\
& & & & x & x \\
& & & & & x
\end{array}\right] .
$$

Fste procedimento faz com que a esparsidade seja preservada na matriz triangular supcrior. pois somente a linha té alterada.

De (4.1) • (4.3) pode-se escrever:

$$
P\left(B^{\prime \prime}\right)^{\prime}=Q V^{-1} R^{-1} I^{-1}
$$

Alternativamente, utilizando a definição $V=Q U^{(1)}$ temos:

$$
P\left(B^{\prime 1 j}\right)^{\prime}=Q\left(U^{(1)}\right)^{-1} Q^{-1} R^{-1} L^{1}
$$

De forma geral, sejam $U=V^{(0)}$ e $R-R_{1}$. Fntão. na iteração $t$ do método simplex, a inversa da base $B^{(i)}$ é representada como:

$$
P\left(B^{(1)}\right)^{\prime}=Q^{(1)} \cdots\left(Q^{(1)}\left(V^{(1)}\right)^{-1}\left(R^{(n)}\right)^{1} \cdots\left(R^{(1)}\right)^{-1} L^{-1}\right.
$$




\section{Exemplo 4.1:}

Considere a mesma matriz. I usada no exemplo 3.1, obtida da decomposição LU de uma matrì básicáa $B^{(1)}$.

$$
V-\left[\begin{array}{ccccc}
-1 & & & -6 & \\
& 1 & -2 & 4 & \\
& & 1 & -3 & \\
& & & 2 & \\
& & & & 1
\end{array}\right] .
$$

Como antes, a matriz $B^{(1)}$ é simplesmente a matriz, $B^{(1)}$. com a coluna $t$ referente a variável básica que deixou a base, substituida pela coluna $k$ referente a variável que entrou para a base.

Cono $L^{-1} P B^{(0)}-V$ e $B^{(1)}$ difere de $B^{(0)}$ em apenas uma coluna. segue que $L^{-1} P B^{(1)}$ coincide com $U$ exceto na coluna alterada. Assim, esta coluna de $L^{-1} P B^{(1)}$ contém $a^{i}-L^{-1} P a_{k}$ que, neste estágio do método simplex, já foi calculada quando da determinação da direção simplex.

Assim. considerando $f=2$ teremos a matriz $L^{-1} P B^{(1)}$ :

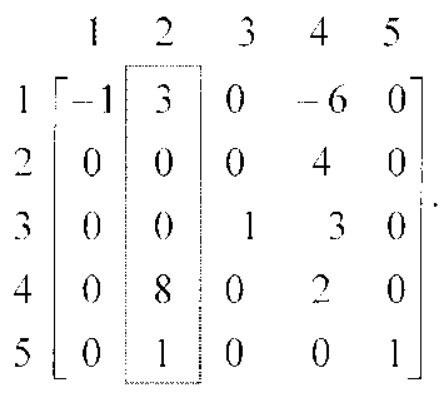

Aplicamos o método proposto por Forrest-Tomlin [15」 para atualizar a inversa da base.

Supondo que foi a segunda variável básica que deixou a base, a segunda coluna da matriz acima é a coluna espeto e, portanto, temos:

$$
z^{2}=\left(\begin{array}{l}
3 \\
0 \\
0 \\
8 \\
1
\end{array}\right)
$$


O primeiro passo é colocar a coluna espeto na última posição da matriz e mover as colunas 3, 4 e 5 uma posição para a esquerda. obtendo a matriz $/ /$ na forma subtriangular superior:

$$
H-\begin{array}{r}
1 \\
2 \\
3 \\
4 \\
5
\end{array}\left[\begin{array}{ccccc}
-1 & & -6 & 3 & 3 \\
& -2 & 4 & \\
& 1 & -3 & \\
& & 2 & & 8 \\
& & & 1 & 1
\end{array}\right] .
$$

A matriz de permutação () é a seguinte:

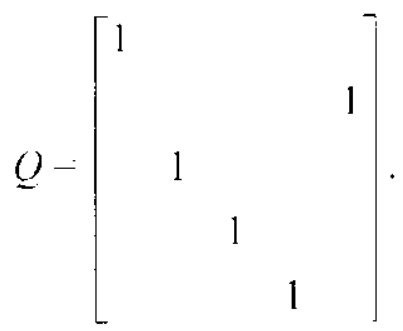

O próximo passo é colocar a matriz $I I$ na forma triangular superior. Para isto, eliminamos os elementos da linha 2, nas colunas 3, 4 e 5 multiplicando a esquerda de $/ /$ pela matriz. $R^{-1}$ e realizamos a permutação de linhas para torná-la uma matriz triangular superior.

Como vimos anteriormente.

$$
\begin{aligned}
& R^{-1}=I-\iota_{2} r^{\prime}, \\
& r^{r}=\pi^{\prime} U^{-1}, \\
& \tilde{u}^{\prime}=\left(\begin{array}{llllll}
0 & 0 & u_{23} & u_{24} & u_{25}
\end{array}\right) .
\end{aligned}
$$

Assim,

$$
\begin{aligned}
& u^{T}=\left(\begin{array}{lllll}
0 & 0 & -2 & 4 & 0
\end{array}\right) . \\
& r^{\prime}=\left(\begin{array}{lllll}
0 & 0 & -2 & -1 & 0
\end{array}\right) \\
& R^{-1}=I-e_{2} r^{\prime}=\left[\begin{array}{lllll}
1 & 0 & 0 & 0 & 0 \\
0 & 1 & 0 & 0 & 0 \\
0 & 0 & 1 & 0 & 0 \\
0 & 0 & 0 & 1 & 0 \\
0 & 0 & 0 & 0 & 1
\end{array}\right]-\left(\begin{array}{l}
0 \\
1 \\
0 \\
0 \\
0
\end{array}\right) \cdot\left(\begin{array}{lllll}
0 & 0 & -2 & \cdots & 0
\end{array}\right)=\left[\begin{array}{lllll}
1 & 0 & 0 & 0 & 0 \\
0 & 1 & 2 & 1 & 0 \\
0 & 0 & 1 & 0 & 0 \\
0 & 0 & 0 & 1 & 0 \\
0 & 0 & 0 & 0 & 1
\end{array}\right] .
\end{aligned}
$$


Determinando a matriz l:

$$
V-R^{-1} H=\left[\begin{array}{lllll}
1 & & & & \\
& 1 & 2 & 1 & \\
& & 1 & & \\
& & & 1 & \\
& & & & 1
\end{array}\right]\left[\begin{array}{ccccc}
-1 & & 6 & 3 \\
& -2 & 4 & \\
& 1 & -3 & \\
& & 2 & 8 \\
& & & 1 & 1
\end{array}\right]=\left[\begin{array}{cccc}
-1 & -6 & 3 \\
& & 8 \\
1 & -3 & \\
& 2 & 8 \\
& & 1 & 1
\end{array}\right]
$$

Finalmente, multiplicando a esquerda da matriz $V$ pela matriz de permutação $Q^{-1}$ temos:

$$
J^{\prime \prime}-Q^{-1} V-\left[\begin{array}{ccccc}
1 & & & & \\
& 1 & & \\
& & 1 & \\
& & & 1 \\
& 1 & & &
\end{array}\right]\left[\begin{array}{ccccc}
-1 & & -6 & & 3 \\
& & & 8 \\
& 1 & -3 & & \\
& 2 & & 8 \\
& & & 1 & 1
\end{array}\right]=\left[\begin{array}{cccc}
-1 & -6 & 3 \\
& 1 & -3 & \\
& 2 & 8 \\
& & 1 & 1 \\
& & & 8
\end{array}\right] .
$$

\subsection{Variantes de Bartels-Golub}

As duas variantes do algoritmo de Bartels-(jolub tentam manter tanto a esparsidade existente nas matrizes básicas quanto a estabilidade numérica. A segunda variante é um aperfeiçoamento da primeira e ambas foram propostas por Reid [32].

Estas variantes podem ser usadas quando equações da forma:

$$
\begin{aligned}
& B x_{B} \quad b \\
& B^{T} \lambda=c_{b}
\end{aligned}
$$

precisam ser resolvidas em toda iteração e cada matri $B$, da sequência obtida, difere de sua antecessora em apenas uma coluna, como ocorre no método simplex.

Assim como o algoritmo de Bartels-Golub, estas duas variantes são usadas para que a decomposição LL' da matriz básica não seja feita um todas iterações. Flas fazem atualizações da decomposição L.U inicial até cue uma nova decomposição da base seja requerida e cm seguida, passam a fazer novamente as atualizações desta nova decomposição c assim sucessivamente. até chegar a solução do problema. 


\subsubsection{Variante 1 - Algoritmo Esparso de Bartels-Golub}

Seja $B^{(0)}$ a matriz básica inicial no método simplex. $\Lambda$ plicando a climinação de Gauss em $B^{(0)}$. com linhas c colunas permutadas, obtemos a decomposição L.L desta base, que pode ser expressa algebricamente da seguinte forma:

$$
E_{m}^{(0)} E_{m+(0)}^{(0)} \ldots E_{1}^{(0)} B^{(0)}=P^{(0)} U^{(0)} Q^{(t)}
$$

onde.

- $E_{i n}^{(0)} E_{m-1}^{(0)} \ldots E_{1}^{(0)}=\left(L_{i}^{(0)}\right)^{1}$ :

- Es é uma matri\% elementar que difere da matriz identidade $/ \mathrm{cm}$ apenas um clemento fora da diagonal (os multiplicadores) e, portanto, representa uma sequência de operações elementares por linha:

- Pe $Q$ são matrizes de permutação;

- Lé uma matriz triangular inferior;

- Ué uma matriz triangular superior.

Cada coluna da matriz triangular inferior $I$ pode ser armazenada como um produto de matrizes elementares. Tal formação, pode simplificar bastante muitas manipulações algébricas feitas com a inversa da matriz triangular inferior. É importante ressaltar que, decompor uma matriz triangular inferior em um produto de matrizes elementares não envolve nenhum cálculo extra.

Denotamos por $B^{(1)}$ a matriz básica da itcração seguinte. que difere de $B^{(0)}$ em apenas uma coluna. $A \sin$. $B^{(t)}$ satisfar a equação:

$$
E_{m}^{(0)} E_{m-1}^{(1)} \cdots E_{1}^{(1)} B^{(1)}=P^{(1)} S^{(1)} Q^{(0)}
$$

onde a matriz $S^{(1)}$ difere da matriz $U^{(0)}$ também por somente uma coluna, a mesma em que $B^{(0)} \subset B^{(1)}$ diferem.

A matriz $S^{(1)}$ possui a seguinte forma: 


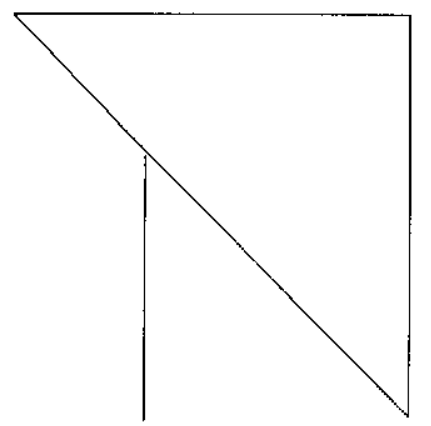

Figura 4.2 - Matriz . S" com coluna espeto

Observando a figura 4.2, vemos que a coluna da matriz. $S^{(1)}$ que difere da matriz $U^{(0)}$ possui elementos não nulos abaixo da diagonal, destruindo a triangularidade de $U^{\left.x^{0}\right)}$. Como vimos anteriormente. Bartels e Golub sugeriram fazer permutaçôes de colunas movendo a coluna espeto para a última coluna da matriz e empurrando as colunas posteriores a espeto uma posição para a esquerda, obtendo uma matrił na forma subtriangular superior, mostrada na ligura abaixo e depois, realizar operações elementares por linha, juntamente com trocas de linhas adjacentes, se necessárias, para restaurar a forma triangular superior.

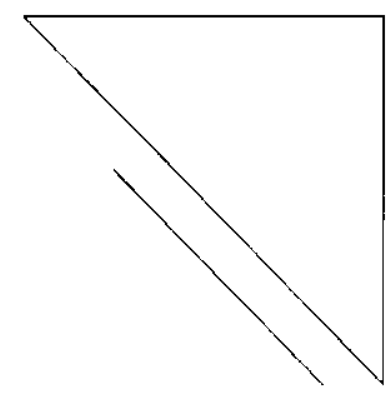

Figura 4.3 - Matriz subtriangular supcrior

Se as operações elementares por linha são escritas como $E_{m i}^{(1)} E_{m}^{(1)} \ldots E_{?}^{(1)}$, a nova fatoração da matrì básica é dada por:

$$
E_{m-1}^{(1)} \ldots E_{1}^{(1)} E_{m-1}^{i(1)} \ldots E_{1}^{(0)} B^{(1)}=P^{(1)} P^{i(1)} Y^{(1)} Q^{(0)} Q^{(1)}
$$

O objetivo principal desta variante é tentar manter a esparsidade presente nas matrizes básicas. Para isto. Reid propôs que, ao fazer a decomposição LL da matriz básica inicial. deve-se utilizar uma heurística de pivoteamento. A heurística que ele utilizou foi a heurística de Markowitz. pelo fato de ter obtido bons resultados com o uso desta heurística em trabalhos 
anteriores. Existe uma outra heurística de pivoteamento, chamada de Grau Mínimo, que também pode ser utilizada. $\Lambda$ mbas heurísticas serão apresentadas no capítulo 5.

Lxiste também a necessidade de se utilizar uma estratégia de pivoteamento para manter a esparsidade, no momento de reduzir a matri $\%$ na forma subtriangular supcrior à forma triangular superior, tentando evitar que preenchimentos excessivos ocorram. Todos os elementos da subdiagonal são nào nulos, pois eles eram elementos da diagonal na matriz triangular superior anterior, mas é provável que muitos elementos da diagonal sejam nulos devido à esparsidade. Se isto ocorrer, muitos passos da redução consistirá em fazer apenas trocas de linhas. Agora, se os elementos da diagonal e subdiagonal são não nulos, a principio. deveria ser escolhido como pivô aquele que possui menos elementos não nulos cm sua linha. Mas. como é indesejável por motivo de estabilidade numérica ter um pivô muito pequeno, deve-se então, tomar como pivô, o maior dos dois clementos (diagonal e subdiagonal) se o menor dos elementos é menor que uma constante $u, 0<u \leq 1$, vezes o maior elemento. caso contrário, o pivô será o clemento que se encontra na linha que possui um número menor de não nulos.

\section{Exemplo 4.2:}

Seja $B$ uma matriz básica esparsa qualquer, decomposta em LLi utilizando-se a heurística de Markowitz para manter tal esparsidade. em uma certa iteração. Assim, a matriz $B$ pode ser representada da seguinte forma:

$$
B^{(0)}=P^{(0)} E_{1}^{(0)} E_{2}^{(0)} \ldots E_{n i}^{(0)} U^{(0)} Q^{(0)}=P^{(0)} L^{(0)} U^{(0)} Q^{(0)}
$$

Seja $B^{(1)}$ a matriz básica da iteração seguinte, que dilere de $B^{(0)}$ apenas pela coluna que entrou na base nesta iteração. $\Lambda \operatorname{ssim}, B^{(1)}$ satislaz a equação:

$$
B^{(1)}=P^{(3)} L^{(0)} S^{(1)} Q^{(1)}
$$

onde a matriz $S^{(1)}$ difere da matri $U^{(0)}$ somente pela mesma coluna em que $B^{(0)}$ difere de $B^{(1)}$.

Suponha que a matriz. $S^{(1)}$ seja a seguinte matriz: 


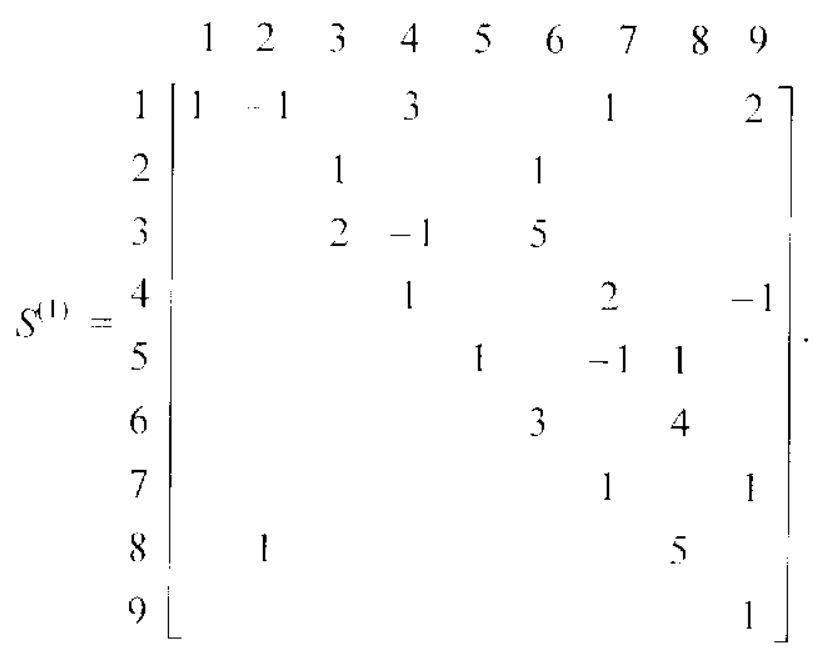

Neste momento, aplicamos a variante 1 para colocar a matriz $S^{(1)}$ na forma triangular superior tentando preservar a esparsidade c obter a decomposição de $B^{(1)}$ apenas atualizando a decomposição já lèta. evitando desta forma. a realização de outra dccomposição o que é caro em termos computacionais.

1- Colocar a matriz $S^{(1)}$ na forma subtriangular superior.

Observando a matriz $S$. vemos que a coluna espeto é a coluna 2. Colocando esta coluna na posição da coluna 9 e movendo as colunas $3,4,5,6,7,8$ c 9 uma posição para a esquerda, obtemos a seguinte matriz na forma subtriangular supcrior:

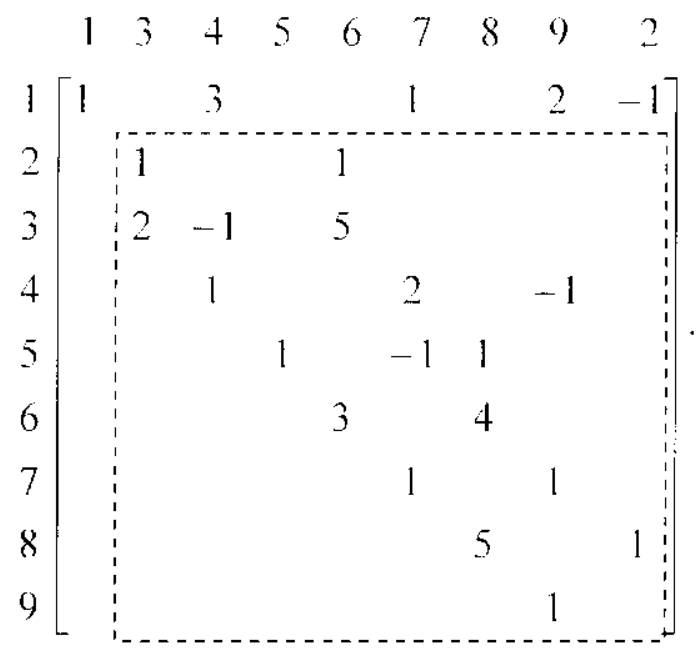

2- Fazer as eliminações para colocar a matriz na forma triangular superior tentando preservar a esparsidade.

Lembrando que antes de fazer a eliminação propriamente dita é preciso escolher qual será o elemento pivô, levando em consideração tanto a questão da estabilidade numérica quanto a questão da esparsidade que deve ser mantida. Temos duas opçóes para a escolha do pivô: 
a) O elemento diagonal é nulo e subdiagonal é não nulo.

Neste caso, o pivô será o elemento da subdiagonal. Basta fàer a troca de linhas.

b) Os elementos diagonal e subdiagonal são não nulos.

Veste caso, será escolhido como pivô o maior dos dois elementos em módulo se menor $<u$ maior, onde $0<u<1$, caso contrário, o pivô será o elemento que se encontra na linha mais esparsa. Um valor típico para $u$ é 0.1 Reid [32].

- Pivô na posição $(2,2)$ :

b) Os elementos diagonal e subdiagonal são não nulos.

- maior $=\left|s_{32}\right|=2$, menor,$- s_{22} \mid=1$ (ambos servem como pivô).

- $u^{*} s_{32}=0.1 * 2-0.2<s_{22}-1$.

- Procurando a linha mais esparsa:

Linha 2 - 2 elementos não nulos;

Linha $3=3$ clementos não nulos.

A linha 2 ć mais esparsa, portanto não é preciso trocar as linhas, pois o pivô já está na posição correta. 7.erando a posição $(3,2)$ da matriz temos:

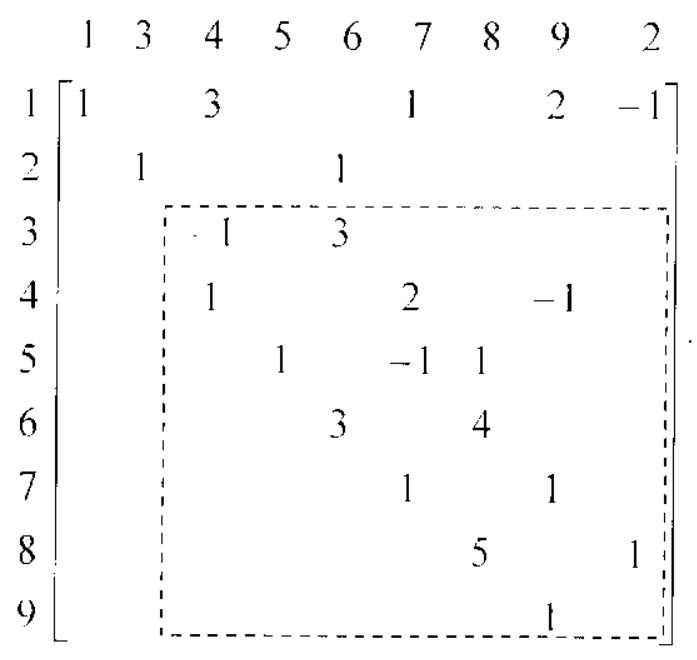

- Pivô na posição $(3,3)$ :

b) Os elementos diagonal c subdiagonal são não nulos.

- maior $-\left|s_{33}\right|=1$. menor $=\left|s_{13}\right|-1$ (ambos servem como pivô).

- $u^{*} s_{33}=0.1 * 1-0.1<s_{43}-1$.

- Procurando a linha mais esparsa:

I.inha 3-2 elementos não nulos;

Linha $4=3$ elementos não nulos. 
$\Lambda$ linha 3 é mais esparsa. portanto não é preciso trocar as linhas, pois o pivô já está na posição correta. 7crando a posição $(4,3)$ da matriz temos:

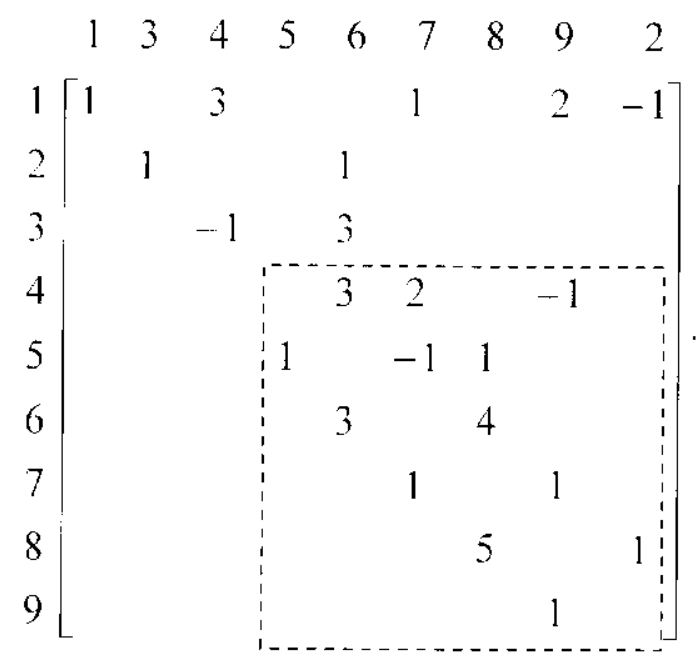

- Pivô na posição $(4,4)$ :

a) O elemento diagonal é nulo e subdiagonal é não nulo.

Trocando a linha 5 com a linha 4 temos:

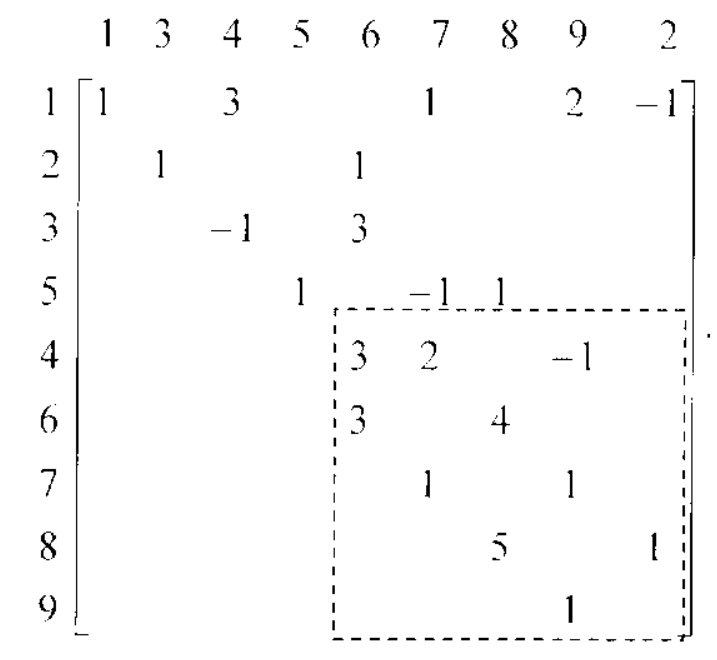

- Pivô na posição $(5,5)$ :

b) Os elementos diagonal e subdiagonal são não nulos.

- maior $=\left|s_{55}\right|=3$, menor ${ }^{-1} s_{65} \mid-3$ (ambos servem como pivô).

- $u^{*} s_{55}=0.1 * 3-0.3<s_{65}=3$.

- Procurando a linha mais esparsa:

Linha 4 - 3 elementos não nulos:

Linha $6=2$ elementos não nulos. 
A linha 6 é mais esparsa. Trocando a linha 6 com a linha 4 e zerando a posição $(6,5)$ da matris, temos:

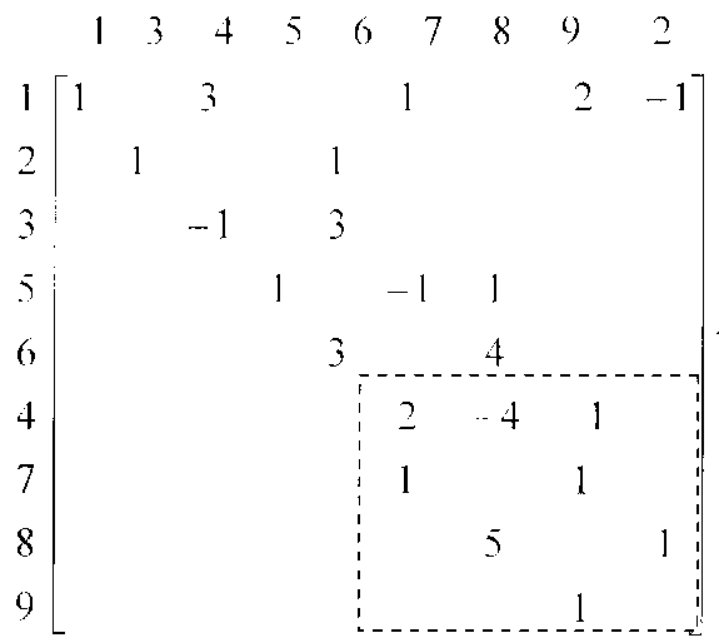

- Pivô na posição $(6,6)$ :

b) Os elementos diagonal e subdiagonal são não nulos.

- maior $=\left|s_{66}\right|=2$, menor ${ }^{-} s_{76}:=1($ ambos servem como pivô).

- $u^{*} s_{660}=0.1 * 2=0.2<s_{76}-1$.

- Procurando a linha mais esparsa:

Linha 4 = 3 elementos não nulos;

Linha $7=2$ clementos não nulos.

$\Lambda$ linha 7 é mais esparsa. Trocando a linha 7 com a linha 4 e zerando a posição $(7,6)$ da matriz temos:

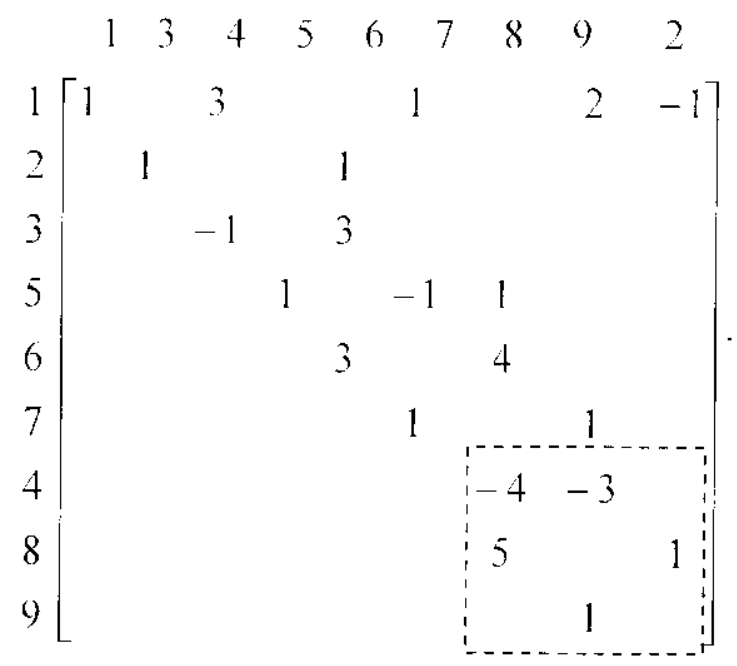


- l'ivo na posiçã̃o $(7,7)$ :

b) Os elementos diagonal e subdiagonal são não nulos.

- maior $-\left|s_{87}\right|=5$. menor $=. s_{77}-4$ (ambos servem como pivô).

- $u^{*} s_{87}-0.1 * 5=0.5<s_{77}-4$.

- Procurando a linha mais esparsa:

Linha $4=2$ elementos não nulos.

Linha $8=2$ elementos não nulos.

Ambas as linhas possuem o mesmo número de não nulos, portanto troca de linha não é necessária. Zerando a posiç̣âo $(8,7)$ da matriz temos:

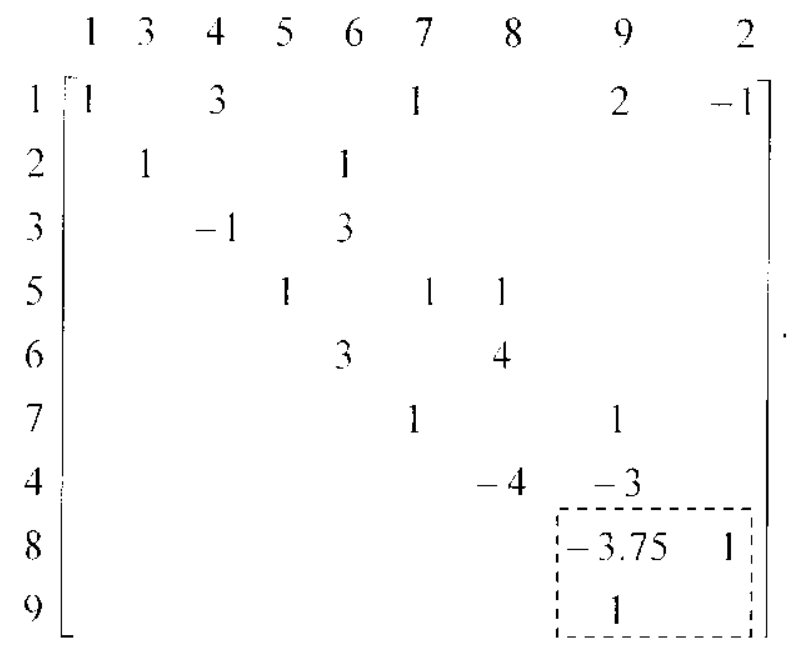

- Pivô na posição (8. 8):

b) Os elementos diagonal e subdiagonal são não nulos.

- nlaior $=\left|s_{88}\right|-3.75$, menor $-\left|s_{58}\right|-1$ (ambos servem como pivô),

- $u * s_{98}-0.1 * 3.75-0.375<s_{58}-1$.

- Procurando a linha mais esparsa:

Linha 8 = 2 elementos não nulos;

Linha $9-1$ elementos não nulos.

A linha 9 é mais esparsa. Trocando a linha 9 com a linha 8 e zerando a posição $(9.8)$ da matriz temos: 


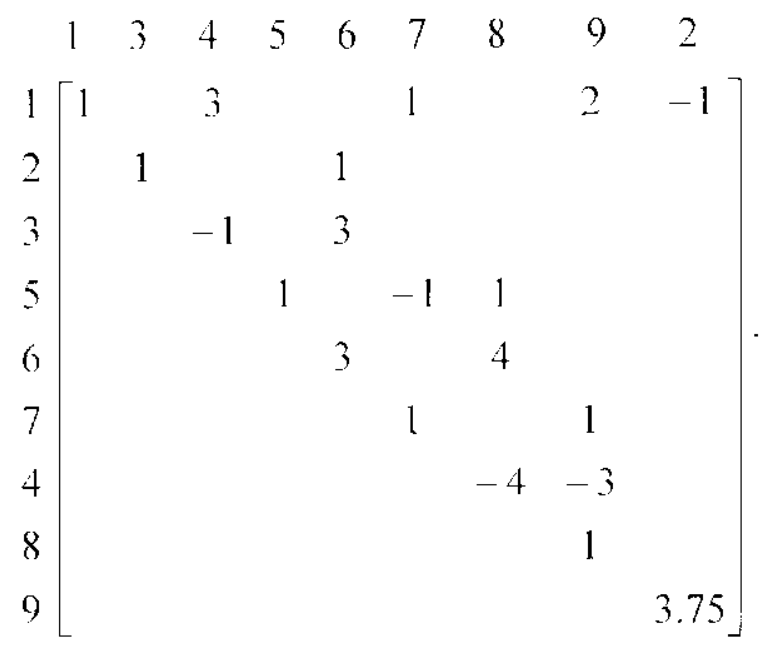

l'inalmente. estamos com a matriz na forma triangular superior como queríamos. Se as operações elementares por linha leitas são escritas como $E_{1}^{(1)} E_{2}^{(1)} E_{3}^{(1)} E_{4}^{(1)} E_{5}^{(1)} E_{6}^{(1)} E_{7}^{(1)}$, e as permutações de linhas e colunas são representadas por $P^{(1)}$ e $Q^{(1)}$ respectivamente, a fatoração da matri básica $B^{(1)}$ é dada por:

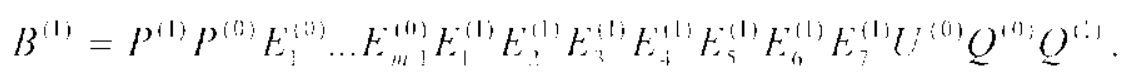

\subsubsection{Variante 2 - $\Lambda$ perfeiçoamento do Algoritmo Esparso de Bartels-Golub}

Esta segunda variante do algoritmo de Bartels-Golub também proposta por Reid é um aperfeiçoamento da variante 1 descrita anteriormente. porque com um pouco mais de permutações de linhas e colunas antes de fazer as eliminações para colocar a matriz novamente na forma triangular superior. tenta-se evitar ao máximo tais eliminações e consegüentemente, os preenchimentos. ou entâo, reduzir o número de elementos na subdiagonal inferior a serem climinados.

Segundo Saunders [35]. alguma vantagem deveria ser tomada do lato que, no caso esparso, a coluna espeto também é bastante esparsa e que, portanto, ao invés de colocar a coluna espeto na última posição da matriz e mover as posteriores a ela uma posição para a esquerda. como é feito na variante 1, a coluna espeto deve ser colocada na posição da linha em que se encontra o último elemento desta coluna. Por exemplo, suponha que a nova matriz $l^{\{1\}}$ a ser colocada na forma triangular superior é de ordem 10 e que a coluna espeto está na coluna 2 e seu último elemento não nulo está na linha 5 . Ao invés de colocar a coluna espeto no lugar da coluna 10 e mover as colunas 3 até 9 uma posição para a esquerda, deve-se colocá-la no lugar da coluna 5 e mover as colunas 3 e 4 uma posição para a esquerda. Desta 
forma. será obtida uma matriz na forma subtriangular superior com menos elcmentos não nulos na subdiagonal, ou seja. apenas não nulos nas colunas 2 a 4 .

Reid. aproveitando a sugestão de Saunders e tentando melhorar ainda mais esta idéia. sugeriu fazer mais algumas permutações de linhas e colunas antes de realizar as eliminações. Vejamos como isto pode ser feito de uma forma mais geral:

Suponha que a coluna espeto esteja na coluna t c que seu último elemento não nulo esteja na linha $q$. Restringimos nossa atenção para a submatriz não triangular formada pelas linhas e colunas de $f$ a q. () objetivo agora. é ir reduzindo o tamanho desta submatriz não triangular para colocar a matriz original na forma triangular superior, ou então, na forma subtriangular superior com menos elementos não nulos na subdiagonal, diminuindo com isso, o número de eliminações a serem realizadas.

O próximo passo é procurar dentro desta submatriz não triangular e a partir da sua segunda coluna. pois a primeira é a coluna espeto, colunas que possuam apenas um elemento não nulo e que este elemento esteja na diagonal. Suponha que a coluna $p$ da submatriz possua tais características, em seguida, deve-se fazer a permutação simétrica (a mesma troca para linbas e colunas) colocando o p-ésimo elemento diagonal na $\ell$-ésima posição. ou seja. a $p$-ésima coluna deve ser colocada na posição da coluna $t$ e as demais colunas $t+1, \ldots, p-1$ são movidas uma posição para a direita e a p-ésima linha deve ser colocada na posição da linha / e as demais linhas $\ell+1 \ldots . . p-1$ são movidas uma posição para baixo. Com estas permutações, a lorma da matriz foi preservada c o comprimento da coluna espeto, agora na coluna $\ell+1$, foi reduzido por um, e conseqüentemente, o tamanho da submatriz não triangular também foi redurido. A coluna espeto passa a ser novamente a primeira coluna da submatriz não triangular. O processo de procurar por colunas que possuem apenas um elemento não nulo na diagonal é repetido na nova submatriz nào triangular de linhas e colunas $t+1$ até $p . \Lambda$ pós isso, nenhuma das colunas $r+2, \ldots, p$ podem possuir apenas um elemento não nulo e na diagonal. então, a procura deve ser iniciada a partir da coluna $p+1$. Tal procura deve ser feita até que não scjam encontradas colunas com estas características (As permutações podem ser fcitas após todas as colunas serem encontradas).

Em seguida, deve ser aplicado um procedimento análogo a este acima às linhas da submatriz não triangular. Suponha que a nova e possivelmente menor submatriz não triangular seja composta por linhas e colunas de até $q$. A procura vai ser feita por linhas que possuam apenas um elemento não nulo e este elemento se encontra na diagonal a partir das linhas $q-1$ ate $t-1$. Se a linha $p$ da submatriz possuir tais características, deve-se farer a 
permutação simétrica (a mesma troca para linhas e colunas) colocando o $p$-ćsimo elemento diagonal na $q$-ésima posição, ou seja, a p-ésima linha deve ser colocada na posição da linha $q$ $\mathrm{c}$ as demais linhas $p+1, \ldots, q$ são movidas uma posição para cima e a $p$-ésima coluna deve ser colocada na posição da coluna $q$ e as demais colunas $p \mid 1, \ldots, q$ são movidas uma posição para csquerda. $\Lambda$ nova procura por outras linhas que tenham um único não nulo e que esteja na diagonal deve ser feita a partir da linha $p-1$ até $\ell$. Com estas permutações, a forma da matri\% também foi preservada co tamanho da submatri\% não triangular poderá ser reduzido por um retirando-se a linha $q$ e coluna $q$ da submatriz não triangular. O processo de procurar por linhas que contêm apenas um elemento não nulo e que esteja na diagonal é feito até que não sejam encontradas linhas com estas características (As permutações novamente podem ser feitas após todas as linhas serem encontradas).

A seguir, deve-se lazer mais algumas permutações de colunas para colocar a submatriz. não triangular novamente na forma subtriangular superior, como antes.

Neste momento, a única coluna na submatriz não triangular que pode ter apenas um elemento não nulo e na diagonal é a última, pois senão a última linha teria estas características. A permutação simétrica novamente deve ser feita para colocar este elemento diagonal no topo da submatriz, da mesma maneira descrita anteriormente. A últimá coluna da nova submatriz não triangular pode de novo possuir um único não nulo $\mathrm{c}$ na diagonal, neste caso. o procedimento anterior deve ser repetido. Liste último passo continua até que não se tenha mais uma submatriz não triangular ou a íltima coluna não possua apenas um elemento não nulo e na diagonal (Mais uma vez todas estas permutações podem ser realizadas juntas posteriormente).

Após todas as permutações possiveis serem realizadas, a matriz $U$ está pronta para que as climinações sejam feitas se estas forem necessárias. com o objetivo de tornar a matriz U triangular superior novamente. caso isto ainda não tenha acontecido. Com a realização de todos estes passos. obtém-se a atualização da decomposição LU feita anteriormente, evitando que uma nova decomposição da matriz básica seja feita, o que representa um grande esforço computacional.

\section{Exemplo 4.3:}

Consideramos a mesma matriz básica $B^{(0)}$, do exemplo 4.2. decomposta $\mathrm{cm} 1, \mathrm{U}$ e também a mesma matriz $B^{(1)}$ que satisfaz a equação $B^{(1)}=P^{(0)} L^{(0)} S^{(1)} Q^{(0)}$.

$\Lambda$ matriz. $S^{(1)}$, como antes, é a seguinte: 


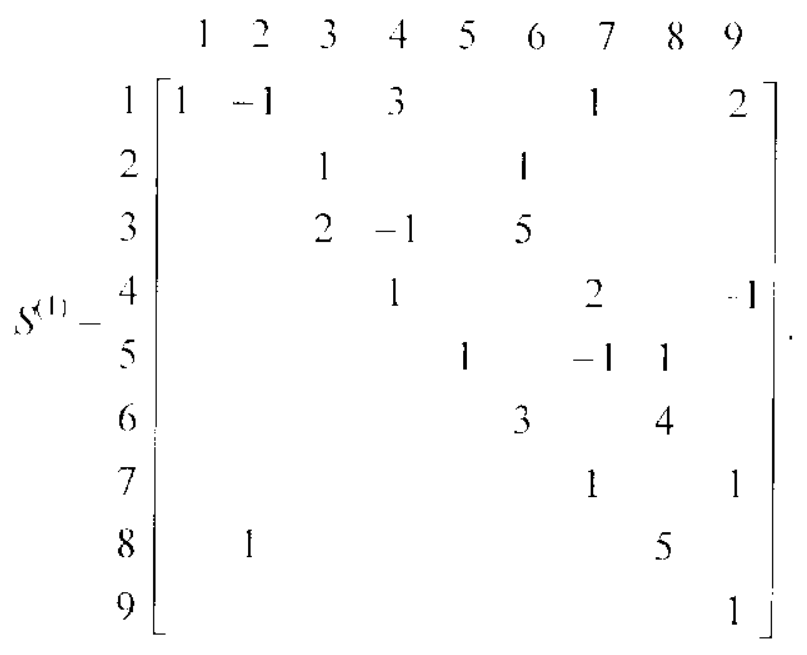

Agora, aplicaremos a variante 2 para colocar a matriz $\$^{1 / 1}$ na forma triangular superior tentando preservar a esparsidade e obter a decomposição de $B^{(1)}$ apenas atualizando a decomposição já feita.

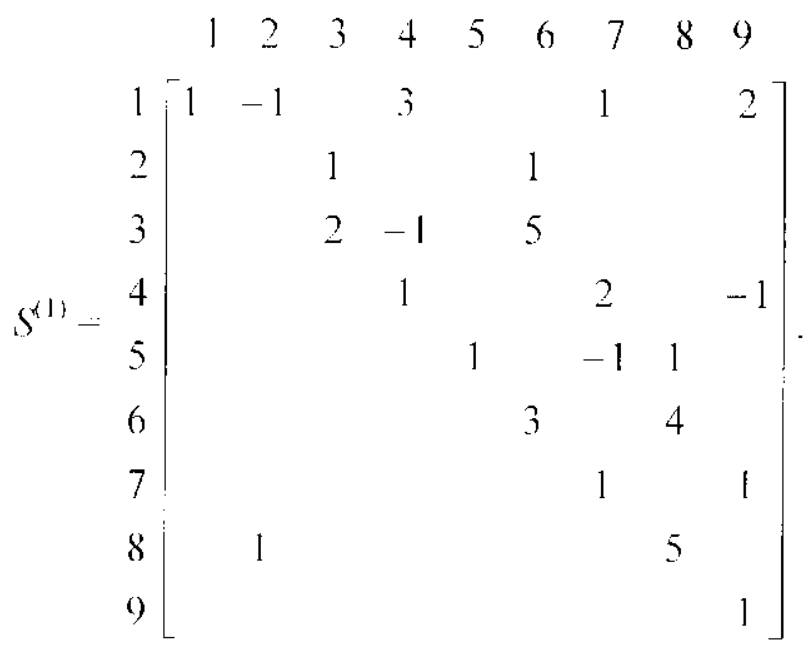

Como podemos observar a matriz $U$ não está na forma triangular por causa da coluna 2 , que é a coluna que acabou de entrar na base. Aplicamos o procedimento descrito acima para colocar esta matriz novamente na forma triangular superior:

1- Determinar a coluna espeto e a submatriz não triangular.

A coluna espeto é coluna $2 \mathrm{e}$ seu elemento não nulo está na linha $8 . \Lambda$ ssim, a submatriz não triangular vai ser formada pelas linhas e colunas de 2 até 8 . Observe que a coluna espeto É a primeira coluna da submatriz não triangular. 


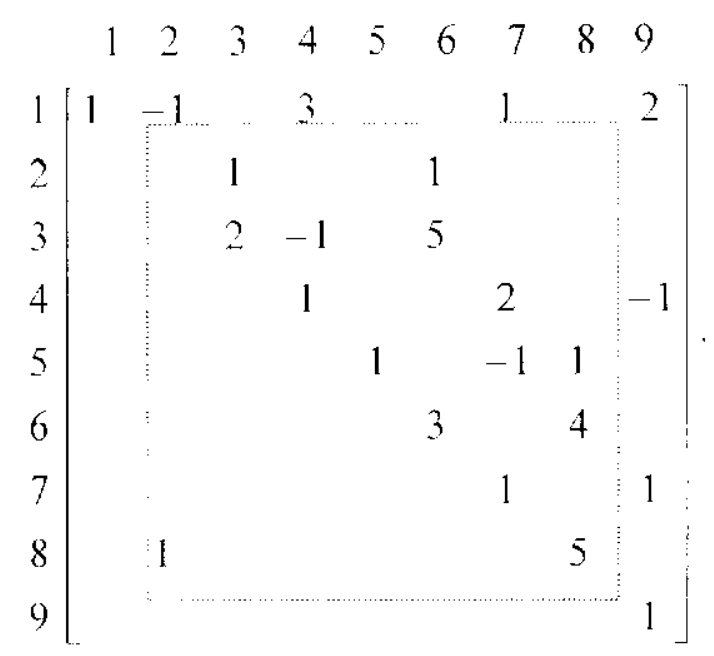

2 - Procurar por colunas que possuam um único elemento não nulo e este elemento esteja na diagonal e fazer as permutações de colunas e linhas para colocar tal elemento no topo da submatris.

Analisando a submatriz não triangular a partir de sua scgunda coluna, vemos que a coluna 5 é uma coluna que possui um único elemento não nulo e na diagonal. Colocando a coluna 5 na posição da coluna 2 e movendo as colunas 2,3 e 4 uma posição para a direita e farando a mesma troca para as linhas colocando a linha 5 na posição da linha 2 e movendo as linhas 2, 3 e 4 uma posição para baixo, temos:

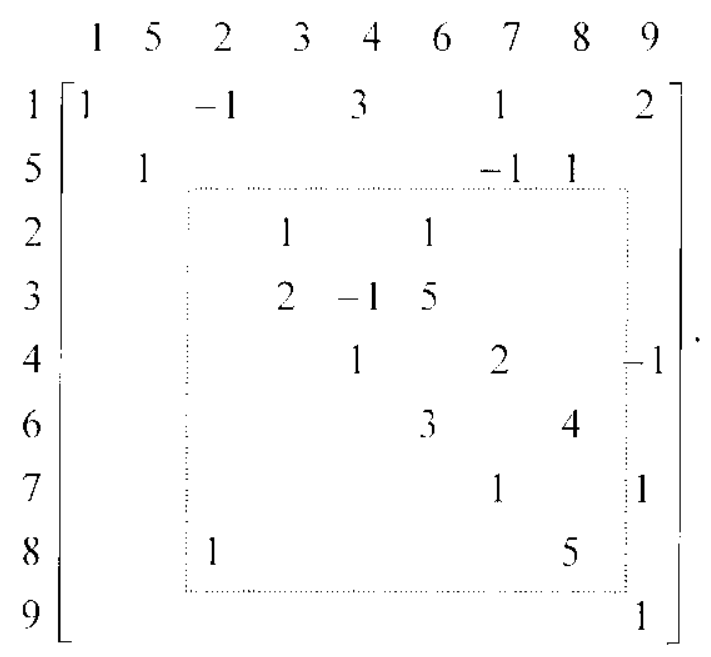

Com estas permutações, a forma da submatriz foi preservada c o comprimento da coluna espeto, agora na terceira coluna da matriz. foi reduzido por um e, consequentemente, o tamanho da submatriz não triangular também foi reduzido.

Continuando a procura por colunas na submatri\% que possuem um único elemento não nulo e que esteja na diagonal, primeiro entre as colunas $3 \mathrm{e} 4 \mathrm{e}$ depois entre as colunas $6,7 \mathrm{e}$ 8, vemos que não existe mais nenhuma coluna com esta característica e portanto, podemos ir para o próximo passo. 
3- Procurar por linhas que possuam um único elemento não nulo e que este elemento esteja na diagonal e fazer as permutações de linhas e colunas para colocar este elemento na última posiçăo da submatriz não triangular.

Iniciando a procura por linhas que possuam um único elemento não nulo e que esteja na diagonal a partir da penúltima linha até a segunda linha da submatriz não triangular, vemos que a linha 7 é uma linha que possui um único elemento não nulo e que está na diagonal. Colocando a linha 7 na posição da linha 8 e movendo a linha 8 uma posição para cima e fazendo a mesma troca para as colunas, colocando a coluna 7 na posição da coluna 8 e movendo a coluna 8 uma posição para a esquerda, temos:

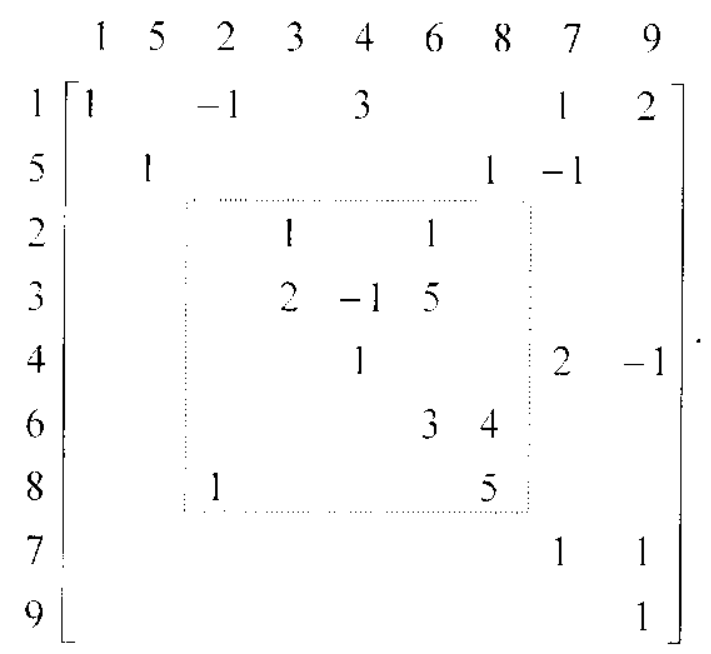

Com estas permutações, a forma da matriz também foi preservada e o tamanho da submatri\% não triangular poderá ser reduzido de um retirando-se a linha 7 e coluna 7 da submatriz não triangular.

A nova procura por outras linhas que tenham un único não nulo e que esteja na diagonal deve ser feita a partir da linha 6 até a linha 2. Podemos observar que a linha 4 é uma linha que possui tais características. Colocando a linha 4 na posição da linha 8 e movendo as linhas 6 e 8 uma posição para cima e fazendo a mesma troca para as colunas, colocando a coluna 4 na posição da coluna 8 e movendo as colunas 6 e 8 uma posição para a esquerda, temos: 


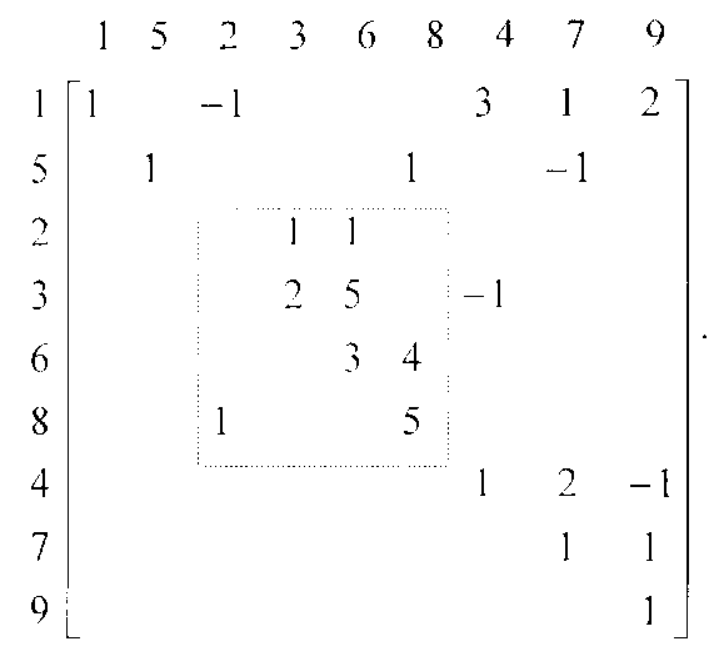

Continuando a procura por linhas que possuem um único elemento e na diagonal, a partir da linha 6 até a linha 2, vemos que não existe mais nenhuma linha na submatriz triangular com estas características c portanto. podemos ir para o próximo passo.

4- Colocar a submatriz não triangular na forma subtriangular superior e verificar se a última coluna possui um único elemento não nulo e que esteja na diagonal. Se isto ocorrer fazer a permutação simétrica para colocar tal clemento no topo da submatri\%. Repetir este passo até que a última coluna não possua apenas um elemento não nulo na diagonal ou não se tenha mais uma submatriz não triangular.

Para deixar a submatriz não triangular na forma subtriangular superior é preciso colocar a coluna 2 na posição da coluna 8 e mover as colunas 3, 6 e 8 uma posição para a esquerda. obtendo-se a seguinte matriz:

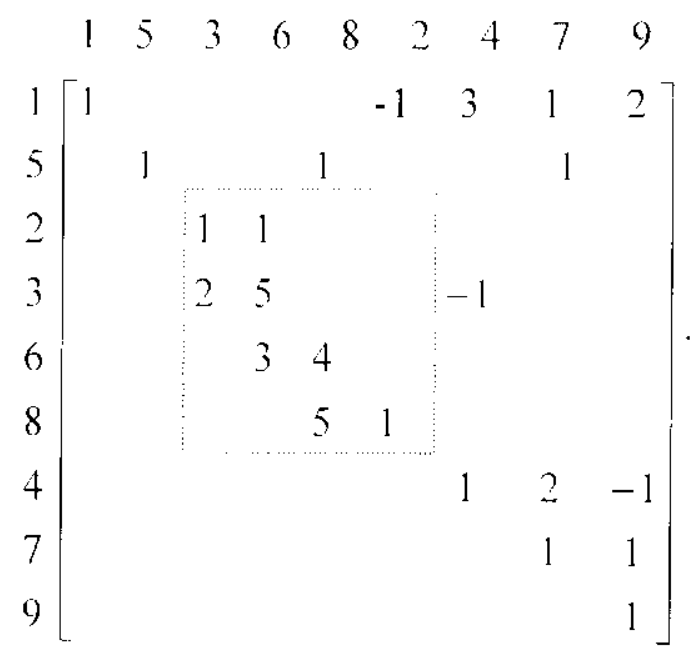

A última coluna da submatriz não triangular, que é a coluna 2, possui um único clemento não nulo e está na diagonal. Colocando a coluna 2 na posição da coluna 3 e movendo as colunas 3,6 e 8 una posição para a direita e fazendo a mesma troca para as 
linhas, colocando a linha 8 que é a última linha da submatriz não triangular, na posição da linha 2 e movendo as linhas 2, 3 e 6 uma posição para baixo, temos:

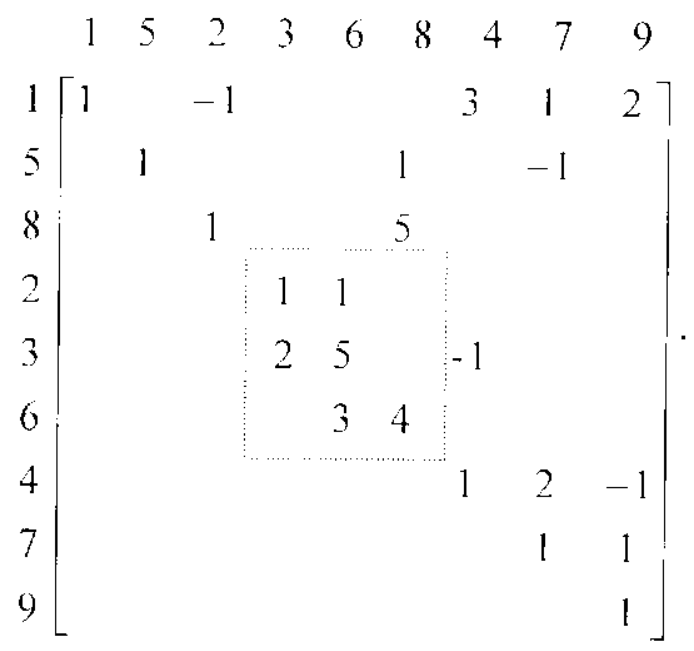

Novamente, a última coluna da submatriz não triangular. que agora é a coluna 8 , possui um único elemento não nulo e está na diagonal. Colocando a coluna 8 na posição da coluna 3 e movendo as colunas 3 e 6 uma posiçăo para a direita e também colocando a linha 6 , que é a última linha da submatriz não triangular, na posição da linha 2 e movendo as linhas 2 e 3 uma posição para baixo. temos:

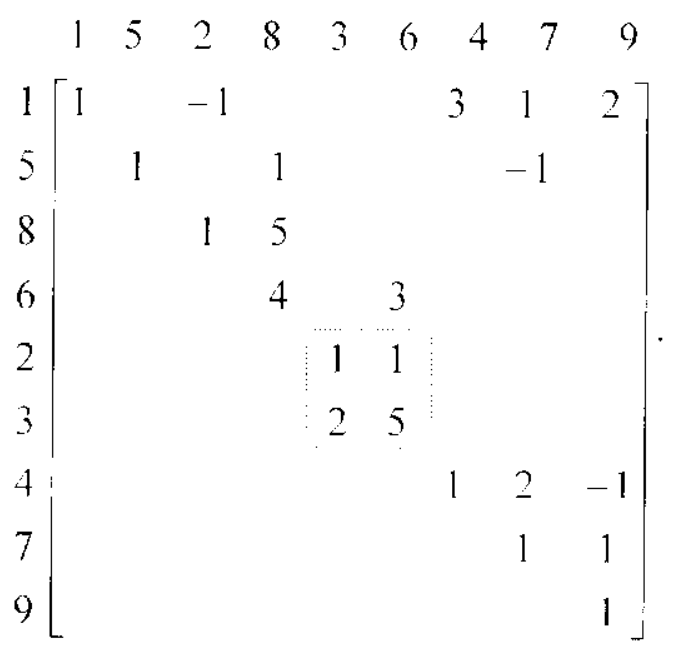

Finalmente, vemos que a submatriz não triangular não tem mais colunas possuam somente um elemento não nulo e na diagonal. Isso quer dizer que o processo terminou e a matriz resultante está praticamente na forma triangular superior, faltando eliminar apenas um elemento fora da diagonal. 
5 - Fazer as climinações necessárias para colocar a matriz $U$ na forma triangular superior.

Realizando a única sliminação a ser feita na matriz. obtemos a atualização da matriz $U:$

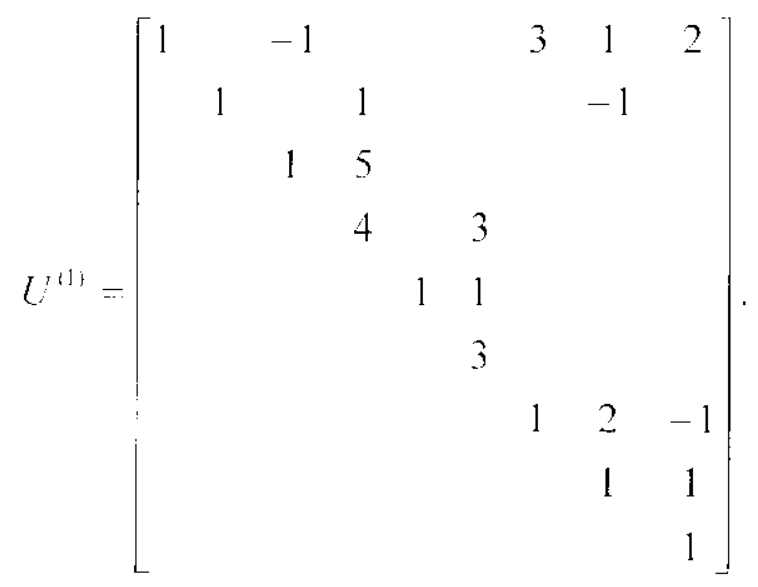

Finalmente. estamos com a matriz na forma triangular superior como queríamos. Se a operação clementar por linha feita é escrita como $E_{1}^{\prime \prime \prime}$. e as permutações de linhas c colunas são representadas por $P^{(1)}$ e $Q^{(1)}$ respectivamente. a fatoração da matri\% básica $B^{(1)}$ é dada por:

$$
B^{(1)}=P^{(1)} P^{(1)} F_{1}^{(i)} \ldots F_{i n ! 1}^{(0)} E_{1}^{(1)} U^{(i)} Q^{(1)} Q^{(1)}
$$




\section{Capítulo 5 - Heurísticas de Pivoteamento}

\subsection{Introdução}

Neste capítulo, serão apresentadas duas heurísticas de pivoteamento que são utilizadas junto com a decomposição LU com o objetivo de preservar a esparsidade existente nas matrizes básicas no método simplex. A primeira heurística descrita é a de Markowitz [26]. Tal heurística é simples e. apesar de muitas outras heurísticas terem sido desenvolvidas e implementadas, ainda é considerada bem eficiente (Duff [13]). A segunda heurística é a de Grau Mínimo (Vanderbei [41]), também bastante simples e possui a vantagem de ser de implementação fácil.

\subsection{Heurística de Markowitz}

Uma das mais antigas heurísticas de selecionar um elemento pivô, na área de esparsidade é devido a Markowitz [26]. A heurística de Markowic possui uma estratégia cficiente para manter a esparsidade de uma matriz quando está sendo feita a decomposição LU, é uma das mais usadas na prática e apresenta resultados bem satisfatórios (Dufl [13]).

A heuristica de Markowitz pode ser descrita da seguinte maneira:

A cada passo, seleciona-se como pivó um elenento que pertença a submatriz em que está sendo leita a eliminação, que chamamos de submatriz ativa. Suponha que a eliminação Gaussiana se encontra no passo $t$, a matriz original (quadrada) tem dimensão $m$ a submatriz ativa é de ordem $(m-l), r_{j}^{(n)}$ é o número de elementos não nulos encontrados na linha $i$ dentro da submatriz e " "é o número de elementos não nulos encontrados na coluna $j$ da submatriz. Scja $A^{(i)}=\left(a_{1 /}^{(n !}\right)$ a matri obtida no passo $/$ do método da eliminação de Gauss. A heuristica seleciona como pivô o elemento $a_{p t}^{\prime \prime \prime} \neq 0$ na submatriz ativa que minimize a expressão: 


$$
\left(r_{i}^{(i)}-1\right)\left(c_{i}^{\prime \prime \prime}-1\right)
$$

Se existir uma linha ou uma coluna que possua apenas um elemento não nulo, tal elemento será selecionado como pivo, evitando-se o aparecimento de qualquer novo elemento não nulo.

Assim, a heuristica de Markowitz consiste em:

Para $l=\cdots 1$ até $m-1$ faça:

1. Determinar $r_{i}^{(t)}$. o número de elementos não nulos encontrados na linha $i$, para todas as linhas $i$ da submatriz ativa na iteração $t$.

2. Determinar $c^{n}$, o número de elementos não nulos encontrados na coluna $j$. para todas as colunas $j$ da submatriz ativa na iteração $t$.

3. Determinar $\left(r_{r}^{(n)}-1\right)\left(c^{(i)}-1\right)=\min _{i}\left(r_{i}^{(n)}-1\right)\left(c^{(n)}-1\right)$ para todo $i, j$ tal que $a_{1 \prime}^{(i)} \neq 0$. Se $r^{\prime \prime \prime}=1$ ou $c^{\prime \prime \prime}=1$ este único elemento da linha ou coluna será o pivô.

4. Trocar linhas e colunas para que o pivô $a_{i}^{t / 1}$ seja colocado na posição $(t, t)$.

5. Eletue a eliminação Gaussiana. se necessária. Se $c_{\| !}^{a !}=1$, eliminações não são necessárias.

\section{Exemplo 5.1:}

Considere a seguinte matriz $B$ esparsa, quadrada e de ordem 5 a ser decomposta no produto LU utilizando a heurística de Markowitz:

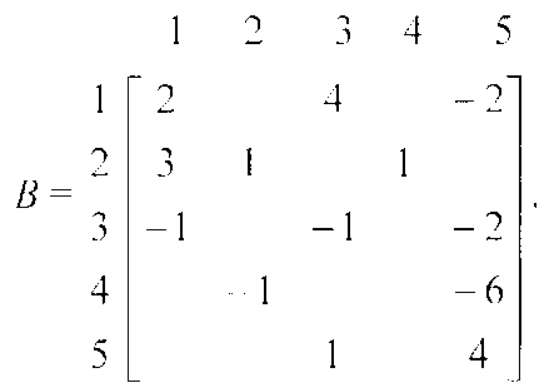


- $\quad$ 1. pivô ná posição (1.1):

1. Determinando $r_{i}^{(1)} . i=1,2,3,4,5$.

$$
\begin{aligned}
& r_{1}^{(1)}=3 ; \\
& r_{2}^{(1)}=3 ; \\
& r_{3}^{(1)}=3 ; \\
& r_{4}^{(i)}=2 ; \\
& r_{5}^{(1)}-2
\end{aligned}
$$

2. Determinando $c_{j}^{(1)}, j=1,2,3,4,5$.

$$
\begin{aligned}
& c_{1}^{(1)}=3 ; \\
& c^{(1)}=2 ; \\
& c_{3}^{(1)}=3 ; \\
& c_{4}^{(1)}=1 ; \\
& c_{5}^{(1)}=4 .
\end{aligned}
$$

3. Determinando o $\min _{i}\left\{\left(r_{i}^{(1)}-1\right)\left(c_{i}^{(1 ;}, \cdots 1\right) i=1,2,3,4,5, j=1,2,3,4,5\right\}$.

$$
\min _{i, j}\left\{\left(r_{i}^{(1)}-1\right)\left(c^{(1)}-1\right) i=1,2,3,4,5, j=1,2,3,4.5\right\}=\left(r_{1}^{(1)}-1\right)\left(c_{4}^{(1)}-1\right)=0 .
$$

Como $c_{4}^{(1)}=1$, basta identificar o único clemento não nulo da coluna 4: $a_{24}$. Assim, a linha 2. deve ser trocada de posição com a linha pivô 1 e coluna 4 deve ser trocada de posição com a coluna pivô 1 .

$\begin{gathered}4 \\ 2 \\ 1 \\ 3 \\ 4 \\ 5\end{gathered}\left[\begin{array}{ccccc}1 & 1 & & 3 & 1 \\ & & 4 & 2 & -2 \\ & & -1 & -1 & -2 \\ & -1 & & & -6 \\ & & 1 & & 4\end{array}\right]$.

4. Como podemos observar, eliminações não são necessárias. 


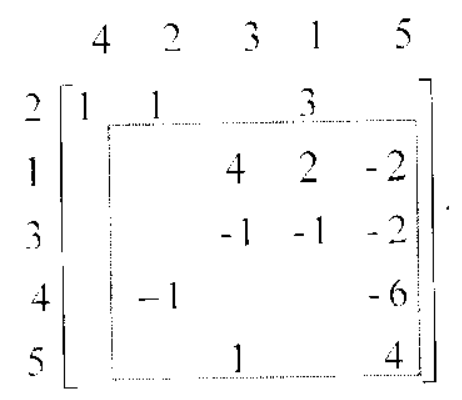

- $\quad t=2$, pivô na posição (2.2):

1. Determinando $r: 2), i-1,3,4.5$.

$$
\begin{aligned}
& r_{r}^{(2)}=3 ; \\
& r_{3}^{(2)}=3 \\
& r_{4}^{(2)}=2 \\
& r_{5}^{(2)}=2 .
\end{aligned}
$$

2. Determinando $c^{(2)}, j, 1,2,3.5$.

$$
\begin{aligned}
& c_{1}^{(2)}=2 ; \\
& c_{2}^{(2)}=1 ; \\
& c_{3}^{(2)}=3 ; \\
& c_{3}^{2(3)}=4 .
\end{aligned}
$$

3. Determinando o $\min _{i, 1}\left\{\left(r_{i}^{(2)}-1\right)\left(c_{j}^{(i)}-1\right) \quad i=1,3,4,5, j=1,2,3,5\right\}$.

$$
\min _{i, 4}\left\{\left(r_{t}^{(2)}-1\right)\left(c_{i}^{(2)} \text { 1) } i \cdots 1,3,4,5, j=1,2,3,5\right\}=\left(r_{4}^{(2)}-1\right)\left(c_{2}^{(2)}-1\right)=0\right.
$$

Como $\mathfrak{c}_{2}^{(2)}=1$, basta identificar o único elemento não nulo da coluna 2: $\mathrm{a}_{42}$. Assim. a linha 4. deve ser trocada de posição com a linha pivô 2 e coluna 2 é a coluna do pivô.

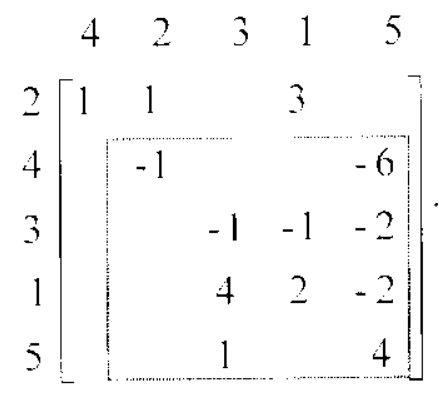


4. Novamente, eliminações não são necessárias.

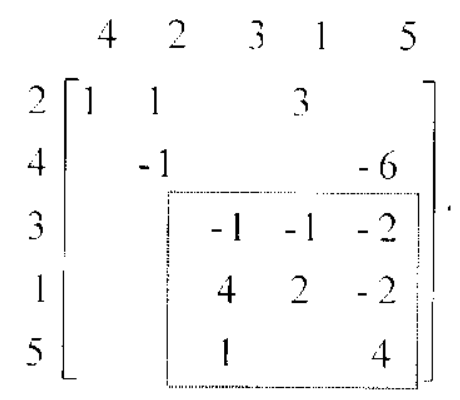

- 1 - 3, pivô na posição $(3,3)$ :

1. Determinando $r_{i}^{\left({ }^{2}\right)}, i=1,3,5$.

$$
\begin{aligned}
& r_{1}^{(3)}=3 \\
& r_{3}^{(3)}=3 \\
& r_{5}^{(i)}=2
\end{aligned}
$$

2. Determinando $c^{(3)}, j=1,3,5$.

$$
\begin{aligned}
& c_{1}^{(3)}=2 \\
& c_{3}^{(3)}=3 \\
& c_{5}^{(3)}=3 .
\end{aligned}
$$

3. Determinando o $\min _{y}\left\{\left(r_{i}^{(3)}-1\right)\left(c_{j}^{: !}-1\right) i=1,3,5, j=1,3,5\right\}$.

$$
\min _{i, j}\left\{\left(r_{i}^{(3)}-1\right)\left(c_{i}^{(3)}-1\right) i=1,3,5, j=1,3,5\right\}=\left(r^{(3)}-1\right)\left(c_{1}^{(3)}-1\right)=2
$$

O elemento a l é o pivô. Assim, a linha 1 deve ser trocada de posição com a linha pivô 3 c a coluna 1 deve ser trocada de posição com a coluna pivo 3 .

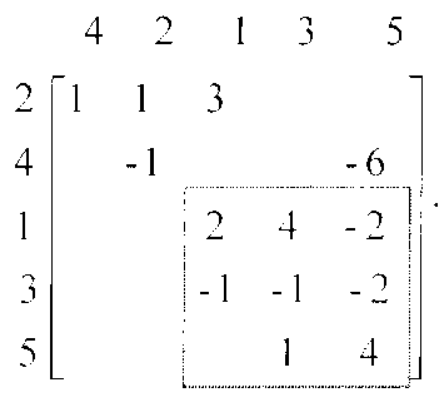


4. Tietuando a eliminação Gaussiana temos:

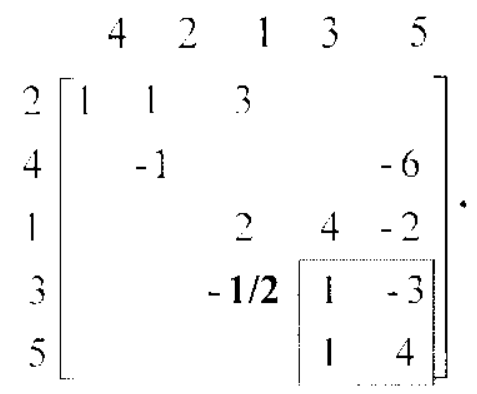

- 1:-4, pivô na posição $(4,4)$ :

1. Determinando $r_{i}^{(4)}, i=3,5$.

$$
\begin{aligned}
& r_{3}^{(+)}=2 \\
& r_{3}^{(1)} 2
\end{aligned}
$$

2. Determinando $c^{(t)}, j=3,5$.

$$
\begin{aligned}
& c^{\prime \prime}=2 ; \\
& c_{5}^{+}=2
\end{aligned}
$$

3. Determinand o $\min \left\{\left(r_{i}^{(1)}-1\right)\left(c_{i}^{(+)}-1\right) i-3,5, j=3,5\right\}$.

$$
\min _{i, j}\left\{\left(r_{i}^{(+)}-1\right)\left(c_{i}^{(+)}-1\right) \quad i=3,5, j=3,5\right\}=\left(r_{3}^{(+)}-1\right)\left(c_{3}^{(4)}-1\right)=1
$$

O clemento $a_{3}$ c o pivô. l'anto a linha 3 quanto a coluna 3 já estão nas posições corretas e portanto. trocas de linha e colunas não são necessárias.

4. Fletuando a eliminação Gaussiana temos:

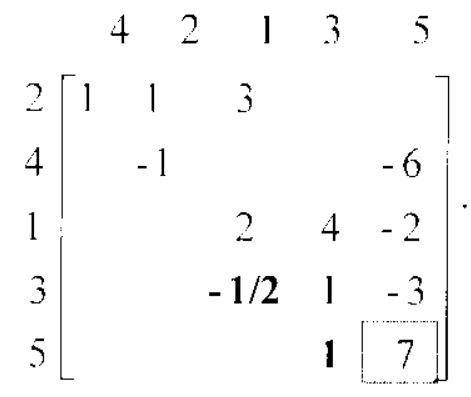

Observação: (Os valores em negrito nas matrizes sâo os multiplicadores utilizados para fazer as eliminações que fazem parte da matriz triangular inferior $L$. 
Jinalmente. a decomposição LU da matriz $B$ é a seguinte:

$$
\begin{aligned}
& B=P L U Q . \\
& {\left[\begin{array}{ccccc}
2 & & 4 & & -2 \\
3 & 1 & & 1 & \\
-1 & & \cdots 1 & & -2 \\
& -1 & & & -6 \\
& & 1 & & 4
\end{array}\right]-P\left[\begin{array}{ccccc}
1 & & & \\
1 & & & \\
& & 1 & & \\
& -1 / 2 & 1 & \\
& & 1 & 1
\end{array}\right] \cdot\left[\begin{array}{ccccc}
1 & 1 & 3 & & \\
& -1 & & -6 \\
& 2 & 4 & -2 \\
& & 1 & -3 \\
& & & & 7
\end{array}\right] Q .}
\end{aligned}
$$

$\Lambda$ s matrizes $P$ e $Q$ são as matrizes de permutações de linhas e colunas respectivamente. realizadas durante a decomposição que podem ser armazenadas como vetores de inteiros conforme visto no capítulo 3.

\subsection{Heurística de Grau Mínimo}

Como vimos anteriormente, a heurística de Markowitz é simples. mas uma dificuldade básica que ela apresenta. é que pode ser muito caro implementá-la (Duff [13]). A heurística de Grau Mínimo. descrita a seguir. é uma outra opção de heurística bastante simples a ser utilizada com o objetivo de manter a esparsidade presente, apresenta também bons resultados (veja os resultados obtidos no capítulo 5) e reduz o trabalho de encontrar o elemento que será o pivô.

A heurística de Grau Mínimo consiste em:

1. Procurar entre as linhas da submatriz ativa aquela que é mais esparsa (digamos a linha $i$ ), ou seja, a linha que possui menos elementos dilerentes de zero:

2. Trocar esta linha com a linha do pivô;

3. Para os elementos não nulos na linha escolhida, selecionar aquele cuja coluna é a mais esparsa, (digamos a coluna $j$ ). ou seja, a coluna que possui menos elementos não nulos na submatriz:

4. Trocar esta coluna com a coluna pivô:

5. Ffetuar a eliminação (jaussiana.

O número de elementos não nulos na submatriz ativa de uma linha e/ou coluna é chamado grau da linha e/ou coluna. 


\section{Exemplo 5.2:}

Consideramos a mesma matriz. $B$ esparsa. quadrada e de ordem 5 do exemplo 5.1 a ser decomposta no produto I I utilizando a heurística do Cirau Mínimo:

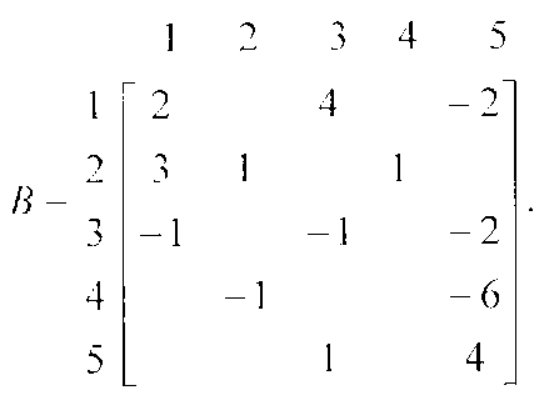

- Pivô na posição (1.1):

1. As linhas 4 e 5 possuem o mesmo grau, ou seja, o número de elementos não nulos na submatriz é igual em ambas as linhas. Quando isso ocorre, escolhe-sc a primeira linha, no caso a linha 4:

2. A linha 4 deve ser trocada de posição com a linha 1:

3. Nesta linha, o elemento 1 na coluna 2 pertence à coluna que possui menos elemcntos não nulos:

4. A coluna 2 deve ser trocada com a coluna 1 .

$\begin{array}{ccccc}2 & 1 & 3 & 4 & 5 \\ 2 \\ 2 \\ 1 \\ 5\end{array}\left[\begin{array}{ccccc}-1 & & & & -6 \\ 1 & 3 & & 1 & \\ & -1 & -1 & & -2 \\ & 2 & 4 & & -2 \\ - & & 1 & & 4\end{array}\right]$.

5. Ffetuando a climinação Gaussiana temos:

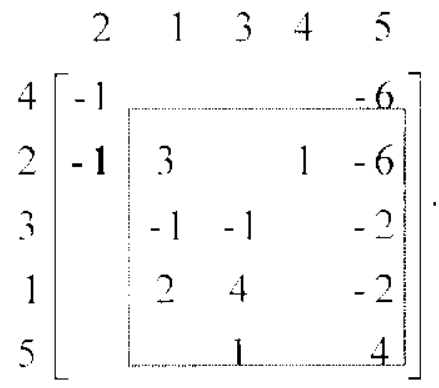


- Pivô na posição $(2,2)$ :

1. A linha 5 possui menos elementos não nulos na submatriz:

2. A linha 5 deve ser trocada de posição com a linha 2;

3. Nesta linha, o elemento 1 na coluna 3 pertence à coluna que possui menos elementos nâo nulos na submatriz;

4. $\Lambda$ coluna 3 deve ser trocada com a coluna 1 ;

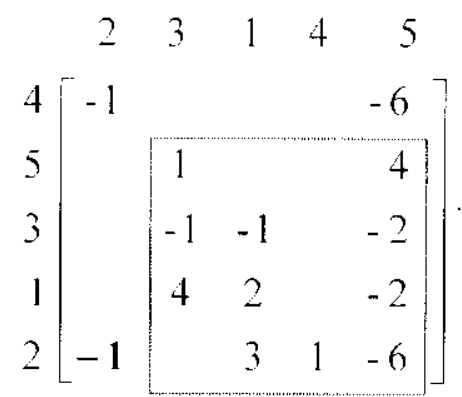

5. Efetuando a eliminação Gaussiana temos:

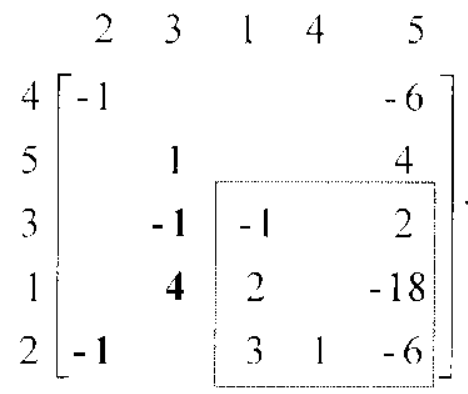

- Pivô na posição $(3,3)$ :

1. A linhá 3 possui menos elementos não nulos na submatriz:

2. A linha 3 está no local correto c trocas não são necessárias;.

3. Nesta linha, o elemento -1 , na coluna 1, pertence a coluna que possui menos elementos não nulos na submatriz:

4. A coluna I já se encontra na posição correta. então não precisa scr trocada:

5. Efetuando a eliminação Gaussiana temos: 


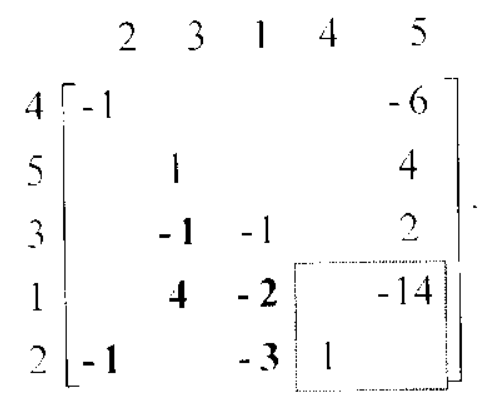

- Pivôna posiçĩo (4. 4):

1. As linhas 1 e 2 também possuem o mesmo grau na submatriz. A linha 1 é escolhida. pois aparece primeiro;

2. Trocas nâo são necessárias;

3. Nesta linha, o elemento -14 na coluna 5 pertence à coluna que possui menos clementos não nulos na submatri\%;

4. A coluna 5 deve ser trocada com coluna 4 :

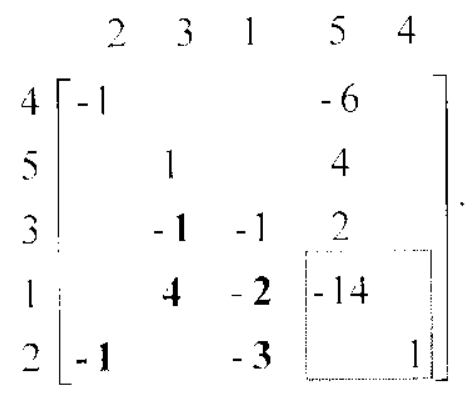

5. Não é necessário efetuar eliminação Gaussiana, pois a submatri\% já está na forma triangular superior.

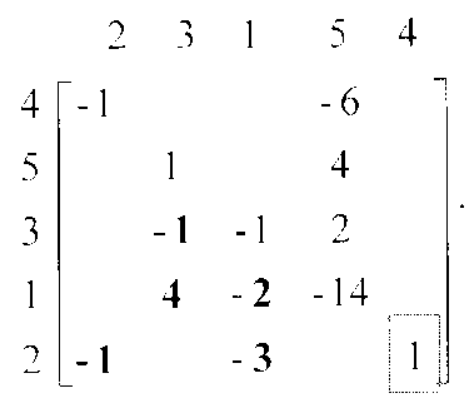

Finalmente, a decomposição LU da matriz $B$ é a seguinte: 


$$
\begin{aligned}
& B=P L L Q . \\
& {\left[\begin{array}{ccccc}
2 & 0 & 4 & 0 & -2 \\
3 & 1 & 0 & 1 & 0 \\
-1 & 0 & -1 & 0 & -2 \\
0 & 1 & 0 & 0 & -6 \\
0 & 0 & 1 & 0 & 4
\end{array}\right]=P\left[\begin{array}{ccccc}
1 & 0 & 0 & 0 & 0 \\
0 & 1 & 0 & 0 & 0 \\
0 & -1 & 1 & 0 & 0 \\
0 & 4 & -2 & 1 & 0 \\
-1 & 0 & -3 & 0 & 1
\end{array}\right] \cdot\left[\begin{array}{ccccc}
-1 & 0 & 0 & -6 & 0 \\
0 & 1 & 0 & 4 & 0 \\
0 & 0 & -1 & 2 & 0 \\
0 & 0 & 0 & -14 & 0 \\
0 & 0 & 0 & 0 & 1
\end{array}\right] Q .}
\end{aligned}
$$

Esta decomposição Ll! possui 8 elementos diferentes de zero fora da diagonal. Se tivéssemos feito a decomposição LU usual, sem utilizar a heurística do Grau Mínimo, teríamos obtido 12 elementos não nulos fora da diagonal. Com isso, foi reduzido 33\% o número de elementos não nulos lora da diagonal. Para matrizes de ordem muito maior que a matri\% usada nesse exemplo, a reduçào pode ser bem mais significativa.

Observação: Quando uma heurística de pivoteamento é utilizada para fazer a decomposição L.. I da matriz de um sistema linear a ser resolvido, por exemplo, o sistema $B x_{b}-b$ (soluçâo básica primal), o primeiro passo a ser feito. é permutar as linhas de $b$ de modo que fiquem de acordo com as linhas de $L$, ou seja. deve-se fazer $P h$ conforme descrito no capitulo 3. Após isso, a substituição progressiva pode ser realizada para resolver $L y=P b$ e a substituição regressiva deve ser leita para resolver $t / x_{/ 3}=y$. linalmente, as coordenadas do vetor $x_{b}$ devem ser rearranjadas de acordo com a matriz de permutação de colunas $Q^{\prime \prime}$ conforme descrito no capitulo 3.

No capítulo 6 apresentamos os resultados computacionais obtidos com cada uma destas heurísticas aplicadas em algumas classes de problemas esparsos. 


\section{Capítulo 6 - Implementações e Experimentos Computacionais}

\subsection{Introdução}

Neste capitulo, serão apresentados: a estrutura de dados utilizada nas implementações, os geradores utilizados para realizar os testes computacionais c também. os resultados obtidos com a realização de tais testes.

\subsection{Estrutura de dados}

A escolha da estrutura de dados usada neste trabalho foi feita baseada no artigo do Reid |32|, justamente porque havia a neessidade de se escolher uma estrutura de dados que aproveitasse a esparsidade existente nos problemas e também porque as variantes por ele propostas. que preservam a esparsidade. foram incorporadas ao método dual simplex canalizado com busca linear por partes. Fsta estrutura não ć exatamente a mesma que Reid $132 \mid$ utilizou. mas a idéia é ben parecida. mesmo porque desde quando as variantes loram implementadas, no início da década de 70 , houve uma boa evolução nos computadores. Nesta época. cra frequente o uso de arquivos para armazenar matrizes e vetores e atualmente isto não é mais necessário.

As implementaçòes tanto dos geradores quanto do método dual simplex canalizado com busca linear por partes juntamente eom as heuristicas de Makowitz e Grau Minimo e as duas variantes de Reid foram feitas em linguagem $\mathrm{C}$. 


\subsubsection{Descrição da estrutura de dados:}

Para armazenar uma matriz esparsa são usados 2 vetores de ponteiros (rows e cols) alocados dinamicamente, cujos tamanhos são. respectivamente, o número de linhas $(n r)$ c o número colunas (nc) da matri\%. IIm destes vetores contém ponteiros que apontam para listas (list) duplamente encadeadas que representam as linhas da matriz e o outro vetor, também contém ponteiros que apontam para listas duplamente encadeadas que representam as colunas da matri\%. Fstas listas, por sua ve\% contêm ponteiros que apontam para o primeiro elemento não nulo de cada linha ou coluna (head) e um inteiro (couml) que indica quantos elementos não nulos existem na linha ou coluna. F. finalmente, cada clemento não nulo da matriz. é representado por um nó (list_element) que contém um ponteiro que aponta para o elemento anterior (prev), um ponteiro que aponta para o próximo elemento (nexi), um ponteiro para o valor do elemento (valuc) e um inteiro (pos) que guarda a posição que o elemento ocupa na linha ou coluna.

Lista estrutura de dados, em linguagem C. é representada da seguinte forma:

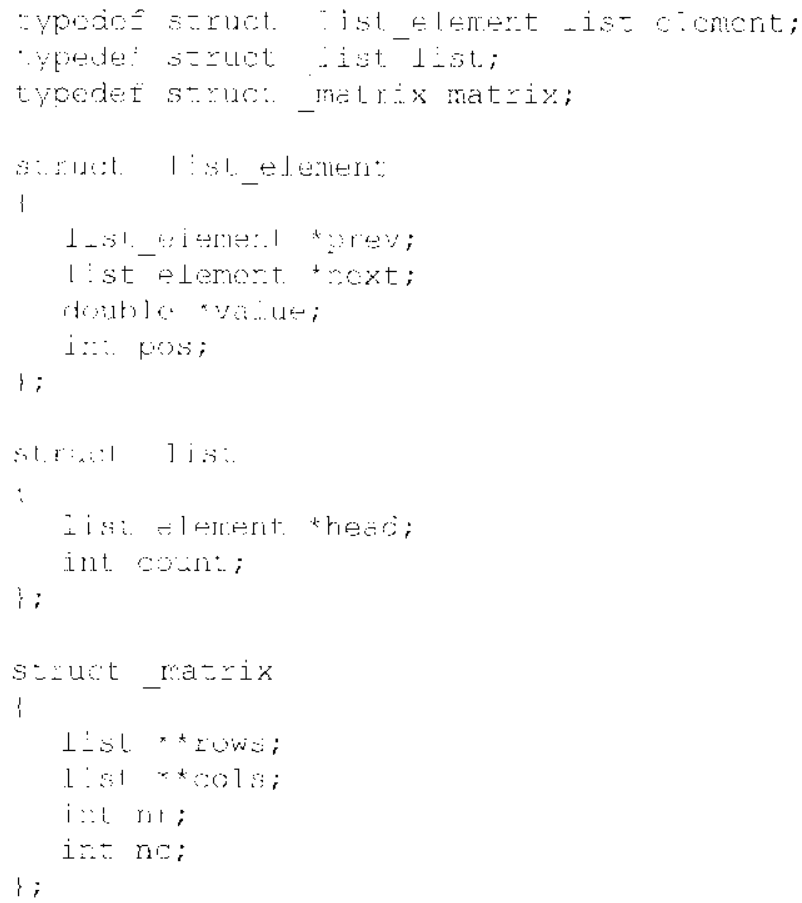

Esta estrutura de dados foi escolhida por simplificar bastante a implementação. uma vez que cla permite fácil acesso aos elementos tanto por linha quanto por coluna, importante para as heuristicas de pivoteamento e formas de atuali/açào da decomposição L.J. (iraficamente, pode ser representada da seguinte forma: 
- matrix:

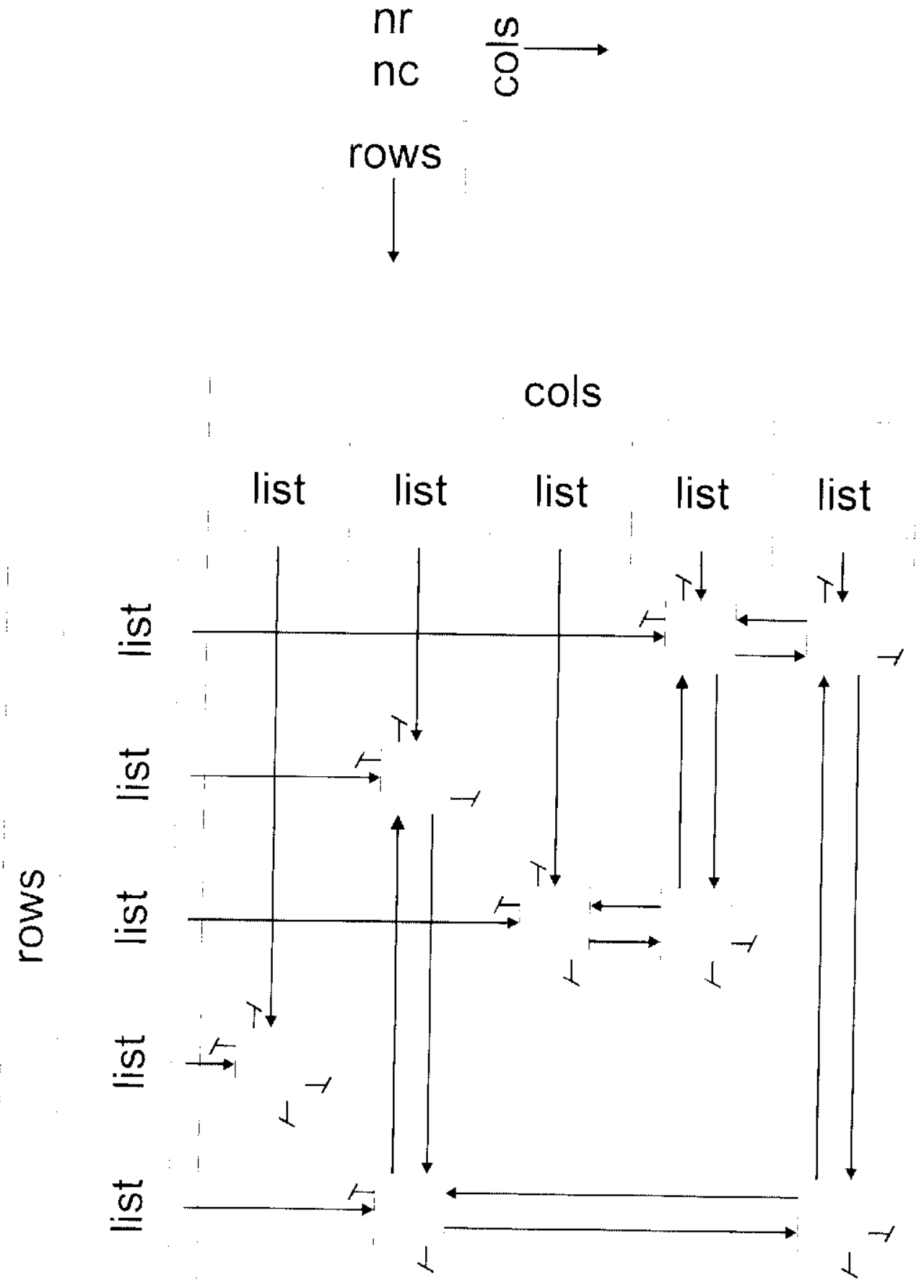

Figura 6.I - Representaçào da matris. 
- list:

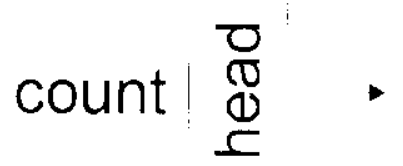

Figura 6.2 - Representação da lista

- list_clement:

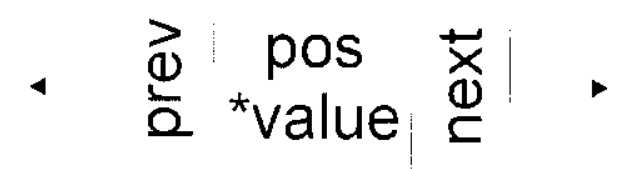

Figura 6.3 - Representação do elemento para linha

\author{
prev \\ pos \\ *value
}

next

Figura 6. 4 - Representação do elemento para coluna 


\subsection{Geradores}

Para a realização dos testes computacionais foram implementados alguns geradores de problemas de otimização lincar canalizados e esparsos. cuja representação grálica da matriz dos coeficientes são dadas a seguir.

\section{- Gerador 1: Produção}

Fste gerador constrói problemas de otimização linear esparsos que representam problemas da produção, cuja forma geral ì a seguinte:

$$
\begin{aligned}
& \text { Minimizar } \sum_{i=1}^{n} \sum_{i}^{t} c p_{n} x_{n}+c c_{n} k_{n} \\
& \left(x_{n}+E_{n,}:-E_{n}=D_{i n}, \quad i=1 \ldots n, \quad l=1 \ldots T .\right. \\
& \text { Sujeito a } \begin{cases}\sum_{t=1}^{n} b_{1} x_{n} \leq c_{i}, & t=1 \ldots T . \\
x_{t} \geq 0, \quad E_{i} \geq 0, & i=1 \ldots n, \quad t=1 \ldots T .\end{cases}
\end{aligned}
$$

liando:

$$
\begin{aligned}
& H_{1}=\left(x_{1}, \ldots x_{n}\right) \\
& E_{1}=\left(E_{1,} \ldots E_{n}\right)
\end{aligned}
$$

O) problema acima pode ser reescrito da seguinte forma:

$$
\begin{aligned}
& \text { Minimizar } \sum_{i=1}^{f} c p_{i} X_{i}+c_{i} E_{i} \\
& \text { Sujeito a } \begin{cases}X_{i}+E_{i, 1}-E_{i}-D_{i}, & t=1 \ldots T \\
b_{i} x_{i} \leq c_{i}, & t=1 \ldots T \\
x_{i} \geq 0, \quad E_{i} \geq 0, & t=1 \ldots T\end{cases}
\end{aligned}
$$

Os dados gerados são arranjados contorme a matriz abaixo: 


$$
\begin{aligned}
& Y_{1} \quad E_{1} \quad I_{2} \quad E_{2} \quad \cdots \quad X_{r} \quad E_{r} \\
& \begin{array}{lll}
I & -I & =D_{1}
\end{array} \\
& \text { bi } \quad<C_{1} \\
& \begin{array}{llll}
l & -l & -D_{2}
\end{array} \\
& \text { hi }^{\prime} \leq \mathrm{C}_{2} \\
& I \quad I=D \text {, } \\
& b^{\prime} \leq c^{\prime}
\end{aligned}
$$

Figura 6.5 - Matriz dos coeficientes do problema da produção

\section{Variáveis c dados:}

$x_{i t}$ : quantidade de itens do tipo $i$ produzidos no período $\%$

$\boldsymbol{E}_{i t}$ : quantidade de itens do tipo $i$ estocados no final do período $t$.

$c p_{i t}$ : custo unitário de produção do item $i$ no periodo $t$.

$c_{e}$ : custo unitário de estocagem do item $i$ no período $t$.

$b_{i}$ : recurso necessário para a produção de uma unidade do item $i$.

$D_{i t}$ : quantidade demandada do item $i$ no período $t$.

$C_{t}$ : capacidade disponível no período $t$.

$\boldsymbol{n}$ : número de itens.

$T:$ númcro de períodos.

Graficamente, as matrizes dos coelicientes poden ser representadas da seguinte forma:

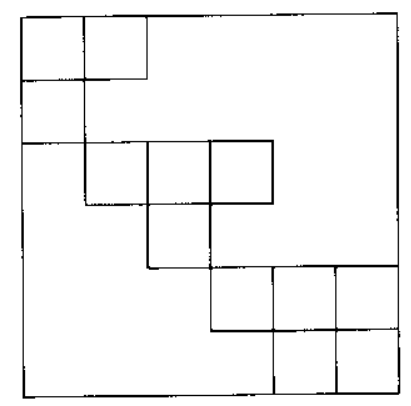

Figura 6.6 - Matriz dos coeficientes do problema da produção

Para gerar o problema, o usuário deve informar o número de produtos $(n)$ a serem produzidos e lambém o número de periodos en que a produção deve ser feita $(T)$. 


\section{rerador 2: Bloco angular}

() gerador bloco angular constrói problemas de otimização linear canalizados e esparsos, cuja matriz dos coeficientes está representada graficamente a seguir. As restrições de acoplamento são representadas por matrizes identidade abaixo de cada bloco.

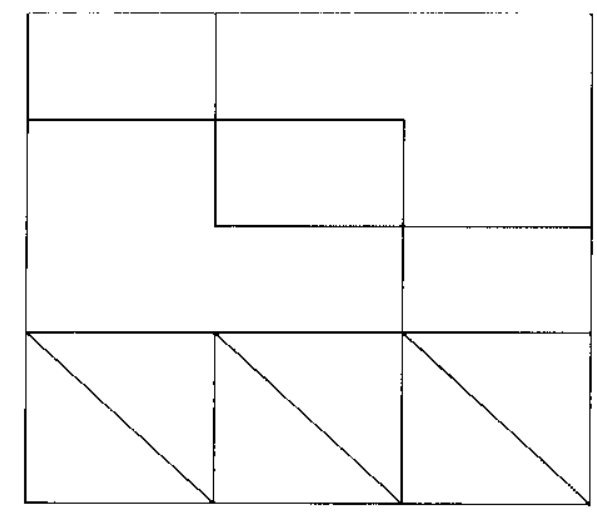

Figura 6.7 - Matriz bloco angular

Para gerar um problema cuja matriz dos coelicientes tem a forma acima, o usuário deve informar a quantidade de blocos e também o número de linhas e colunas de cada bloco. $O$ número de linhas de acoplamento é igual ao número de colunas de cáda bloco.

\section{Gerador 3: Escada}

O gerador escada constrói problemas de otimização linear canalizados e esparsos, cuja matriz dos coeficientes é representada graficamente da seguinte forma:

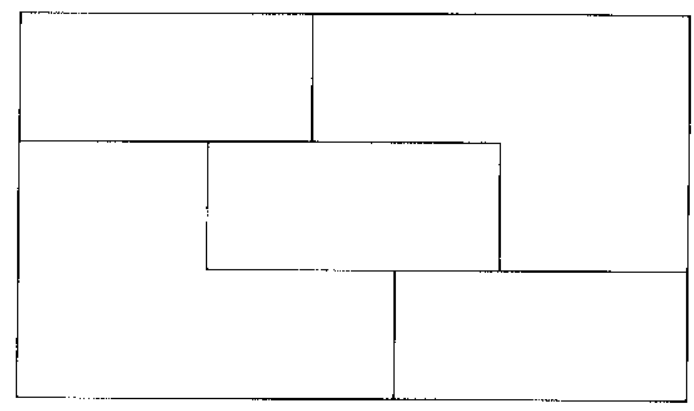

Figura 6.8 - Matriz escada

Para gerar um problema cuja matriz dos coeficientes tem a forma representada acima, o usuário deve informar a quantidade de blocos. o número de linhas e colunas de cada bloco e também o número de colunas de interseção. 


\section{Gerador 4: Bloco angular em vários níveis}

Fste gerador constrói problemas de otimização linear canalizados e esparsos, cuja matriz dos cocficientes é representada gralicamente da seguinte forma:

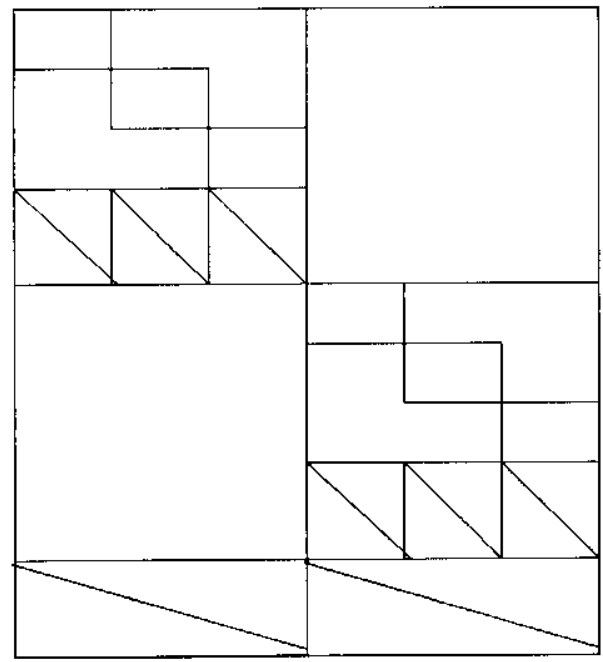

Figura 6.9 - Matriz bloco angulat em vários niveis

Para gerar um problema cuja matriz dos coeficientes tem a forma acima, o usuário deve informar a quantidade de blocos maiores, a quantidade de blocos menores e também o número de linhas e colunas de cada bloco menor. O número de linhas de acoplamento é igual ao número de blocos menores de cada bloco maior.

Lim todos os geradores, o problema gerado é representado de duas formas distintas:

1 - Na primeira, usada pela implementação do método dual simplex canalizado com busca linear por partes. os dados são armazenados em quatro diferentes arquivos do tipo $t x t$ :

- arquivo A: guarda a transposta da matriz dos coelicientes e na primeira linha a ordem desta matriy;

- arquivo c: guarda os custos da função objetivo e na primeira linha o tamanho do vetor:

- arquivo $r$ : guarda na primeira linha o tamanho do vetor. nas posições seguintes os limitantes inferiores das restriçōes e das variáveis;

- arquivo s: guarda na prineira linha o tamanho do vetor, depois os limitantes superiores da restriçòes e das variáveis.

2- Na segunda mancira, o problema gerado é salvo em apenas um arquivo do tipo Ip chamado cplex e apresenta o problema da forma que o software CPLEX consegue ler. Maneira esta, muito parecida com a forma com que os problemas de otimização linear são apresentadas pelos livros. artigos, etc. 
Para um melhor entendimento, abaixo está um exemplo de um problema construído utilizando-se o gerador produção com dois produtos e dois períodos c cujos dados da matrǐ dos cocficientes estão organizados de acordo com a figura 6.6. Os períodos podem representar dias, semanas. etc.

- Forma 1: Método dual simplex canalizado com busca unidimensional linear por partes:

- Matriz dos coeficientes armazenada no arquivo A.tut:

$$
\begin{array}{lllllll}
8 & 6 & & & & & \\
1.00 & 0.00 & 2.20 & 0.00 & 0.00 & 0.00 \\
0.00 & 1.00 & 3.52 & 0.00 & 0.00 & 0.00 \\
-1.00 & 0.00 & 0.00 & 1.00 & 0.00 & 0.00 \\
0.00 & -1.00 & 0.00 & 0.00 & 1.00 & 0.00 \\
0.00 & 0.00 & 0.00 & 1.00 & 0.00 & 1.90 \\
0.00 & 0.00 & 0.00 & 0.00 & 1.00 & 1.23 \\
0.00 & 0.00 & 0.00 & -1.00 & 0.00 & 0.00 \\
0.00 & 0.00 & 0.00 & 0.00 & -1.00 & 0.00
\end{array}
$$

- Vetor dos custos armazenado no arquivo cist:

$$
\begin{gathered}
8 \\
17.12 \\
10.00 \\
0.50 \\
0.60 \\
8.48 \\
16.06 \\
0.30 \\
0.30
\end{gathered}
$$

- Vetores das canalizações das restrições e variáveis armazenados nos arquivos r.txt (limitantes inferiores) estat (limitantes superiores): 


$\begin{array}{cc}r . t x t & s . t x t \\ 14 & 14 \\ 1475.00 & 1475.00 \\ 1089.00 & 1089.00 \\ 0.00 & 7078.28 \\ 1087.00 & 1087.00 \\ 1578.00 & 1578.00 \\ 0.00 & 4006.24 \\ 0.00 & 999999.00 \\ 0.00 & 999999.00 \\ 0.00 & 999999.00 \\ 0.00 & 999999.00 \\ 0.00 & 999999.00 \\ 0.00 & 999999.00 \\ 0.00 & 999999.00 \\ 0.00 & 999999.00\end{array}$

Observação: Alguns valores de $r$ e $s$ são iguais. pois eles se referem à restrição de igualdade do problema.

Forma 2: CPLEX:

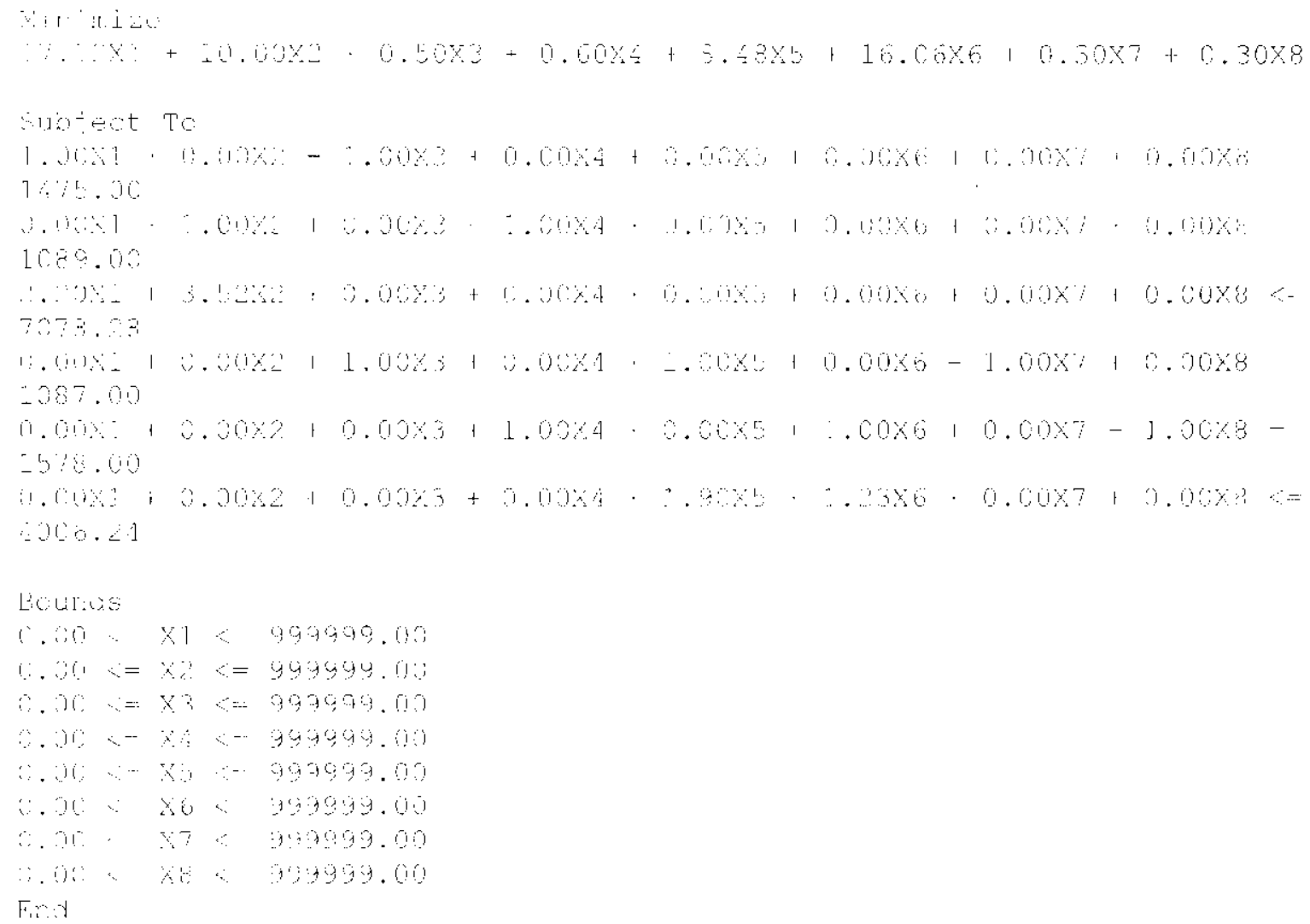




\subsection{Um pouco sobre o programa}

A implementação foi feita em linguagem C utilizando o software Microsoft Visual C+6.0. Neste momento, veremos como utilizar o programa e o que temos no arquivo solução. Uma cópia do exccutável deste programa está em anexo.

$\Lambda$ tela inicial do programa é a seguinte:

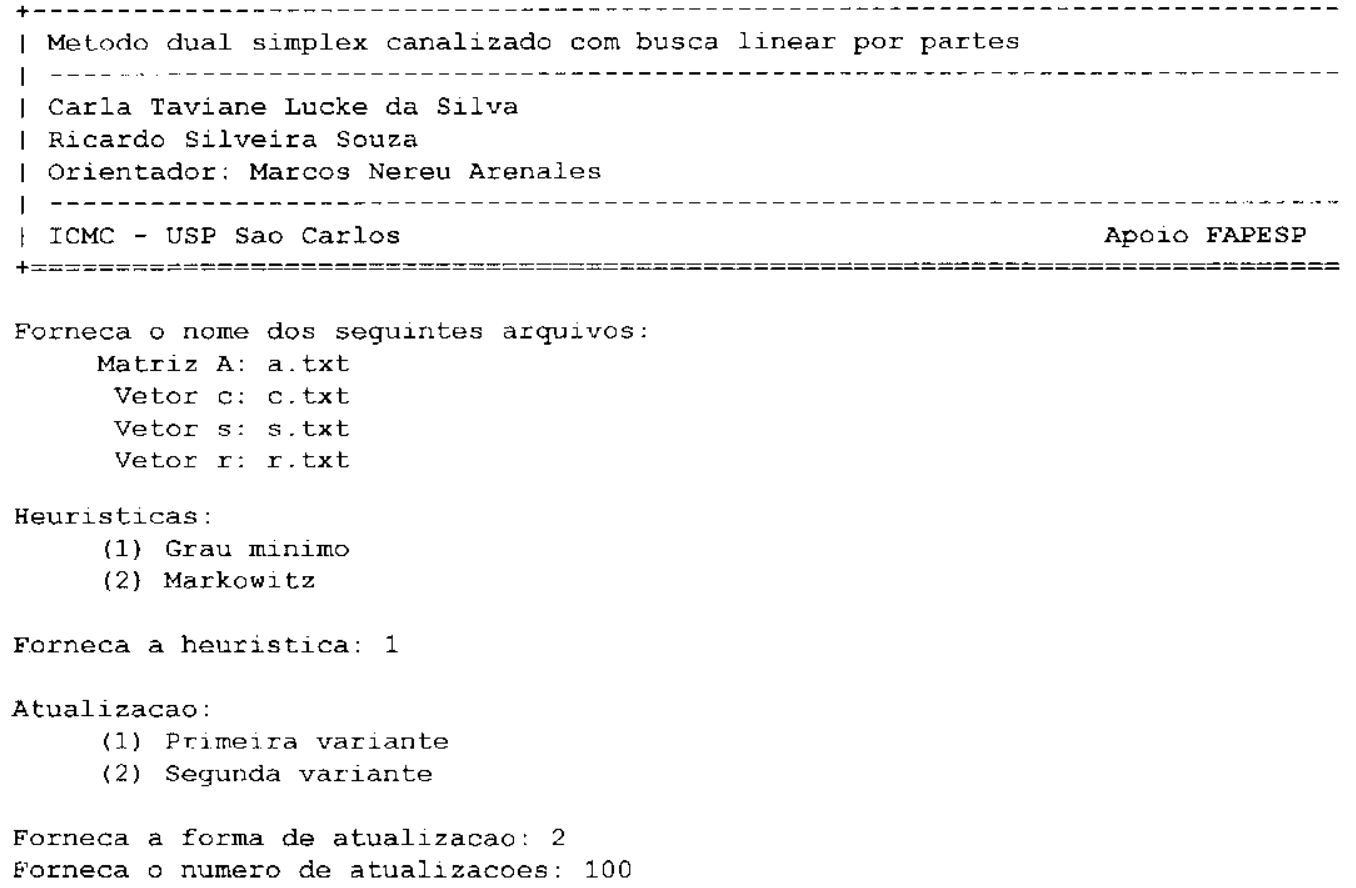

Primeiramente. o usuário deve fornecer os nomes dos arquivos que armazenam o prohlema. onde o arquivo $A$ armazena a matriz dos coeficientes do problema, o arquivo c armazena o vetor dos custos, o arquivo s armazena o vetor dos limitantes superiores das restriçôes e variáveis e o arquivor armazena o vetor dos limitantes inferiores das restriçooes e variáveis. Lim seguida, deve-se escolher qual heurística de pivoteamento vai ser usada: (1) Grau Mínimo e (2) Markowitz. Depois, deve-se informar qual variante vai ser usada para fazer a atualização da decomposição: (1) primeira variante e (2) segunda variante. E por último. o número de atualizações a serem feitas antes de decompor novamente a matriz. hásica. O valor 100 e usado pelo CPI.EX como "default". Alguns testes foram leitos variando-se este valor para o número de atualizaçôes com o intuito de se concluir algo sobre ele. uma ve\% que o número de alualizações feitas interfere no tempo de resolução do problema c também na preservação da esparsidade.

Resolvido o problema. em um arquivo chamado solleçüe pode-se encontrar o valor ótimo da função objetivo e das variáveis. o número de iterações realizadas. o tempo total de

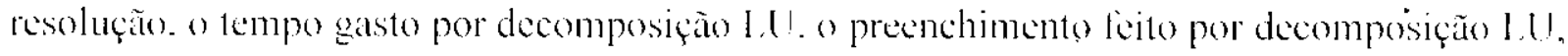
o tempo gasto por atualização e o preenchimento feito por atualização. 


\subsection{Resultados Computacionais}

Os testes realizados neste trabalho foram feitos usando os problemas gerados aleatoriamente pelos quatro geradores descritos anteriormente. cujos tamanhos são pequeno e médio. o número de linhas $(m)$ e colunas $(n)$ variam entre 50 e 3000 aproximadamente e a densidade ( $n z$ ) varia entre $0.2 \%$ e $6 \%$ aproximadamente. São 12 problemas com o número de colunas maior que o número de linhas $(n>m)$ e 12 problemas onde número de linhas é maior que o número de colunas $(m>n)$. Para cada ordem foram resolvidos 10 problemas e calculada a média dos resultados obtidos.

Todos os testes foram realizados cm um computador AMD Atlhon $1800+(1500 \mathrm{MHz})$ com 1GB de R M e um hard disk de 40GB. Foi usado também o compilador Intel para rodar os exemplos, pois o CPLEX para Windows utiliza este compilador a uma ver que os dados obtidos peto dual seriam comparados em relação ao tempo com o CPLI:X era importante estar em condições mais semelhantes possiveis.

A seguir. são apresentados nas tabelas de 6.1 a 6.5 os resultados dos experimentos realizados com problemas construidos pelo gerador escada variando-se os valores para o número de atualizações a serem feitas antes de uma nova decomposição LU da matriz básica $(100, \sqrt{m}, 3 \sqrt{m} / 2$ e $0.1 m)$. Os itens a serem analisados são: número de iterações realizadas, tempo total de resolução, preenchimento feito por atualização, tempo gasto por atualização, preenchimento feito por decomposição e tempo gasto por decomposição.

\begin{tabular}{|c|c|c|c|c|c|}
\hline \multirow[b]{3}{*}{ Problemas } & \multicolumn{4}{|c|}{ Número de Iterações } & \\
\hline & & \multicolumn{4}{|c|}{ Número de Atualizaçōes } \\
\hline & $\%$ nz & 100 & $\sqrt{m}$ & $3 \sqrt{\mathrm{m} / 2}$ & $0.1 \mathrm{~m}$ \\
\hline $50 \times 401$ & 4,24 & 70,5 & 70,7 & 70,6 & 70,6 \\
\hline $100 \times 401$ & 2,24 & 122,3 & 122,8 & 122,8 & $\uparrow 22,8$ \\
\hline $150 \times 401$ & 2.24 & 180,1 & 182,2 & 182 & 182,5 \\
\hline $200 \times 401$ & 1,25 & 211.3 & 211,6 & 211.4 & 211,6 \\
\hline $100 \times 1001$ & $2: 1$ & 148,2 & 148 & 148.3 & 148 \\
\hline $250 \times 1001$ & 0,9 & 307,6 & 310,8 & 311 & 311 \\
\hline $500 \times 1001$ & 0,5 & 519.4 & 525.6 & 525 & 526,2 \\
\hline $200 \times 2001$ & 1,05 & 300,4 & 299.8 & 300,2 & 3004 \\
\hline $500 \times 2001$ & 0,45 & 617 & 619.8 & 618,4 & 618.2 \\
\hline $1000 \times 2001$ & 0,25 & 1050,6 & 1067 & 1069 . & 1050,6 \\
\hline $300 \times 3001$ & 0,7 & 447,2 & $459: 2$ & 455 & 459,3 \\
\hline $750 \times 3001$ & 0,43 & 1008 & 1026.2 & 1023,6 & 10232 \\
\hline $400 \times 51$ & 5.88 & 94,3 & 94,6 & 94,7 & 94,6 \\
\hline $400 \times 101$ & 2,97 & 160,6 & 160,9 & 161 & 161,2 \\
\hline $400 \times 151$ & 1,49 & 222,8 & 224,1 & 224,1 & 224 \\
\hline $400 \times 201$ & 2,97 & 253 & 253,6 & 253,7 & 254 \\
\hline $1000 \times 101$ & 2,65 & 196 & 196,3 & 196,2 & 196 \\
\hline $1000 \times 251$ & 0,6 & 401,5 & 401,7 & 402,2 & 401.5 \\
\hline $1000 \times 501$ & 0,6 & 618,6 & 619,6 & 621,2 & 618,6 \\
\hline $2000 \times 201$ & 1,2 & 403,8 & 406 & 406,6 & 407,4 \\
\hline $2000 \times 501$ & 0,3 & 797,6 & 814,4 & 810 & 810 \\
\hline $2000 \times 1001$ & 0,53 & 1248,8 & 1265,6 & 1260,8 & 1266,2 \\
\hline $3000 \times 301$ & 1,49 & 582,2 & 586 & 587 & 584,4 \\
\hline $3000 \times 751$ & 1 & 1245,8 & 1273,2 & 1263,4 & 1265 \\
\hline
\end{tabular}

Tabela 6.1 - Comparação entre o mumero de iteraçòes para diferentes numeros de atualizaçóes 
Ao analisar a tabela 6.1 vemos que em relação ao número de itcrações os resultados foram próximos em todos os problemas para os quatro valores usados. Mas, o valor 100 foi ligeiramente melhor para a maioria dos problemas.

\begin{tabular}{|c|c|c|c|c|c|}
\hline \multicolumn{6}{|c|}{ Tempo de Resolução em segundos } \\
\hline & \multicolumn{4}{|c|}{ Número de Atualizaçöes } \\
\hline Problemas & $\% n z$ & 100 & m & $3 \sqrt{m} / 2$ & $0.1 \mathrm{~m}$ \\
\hline $50 \times 401$ & 4.24 & 0,176 & 0.27 & 0,238 & 0,321 \\
\hline $100 \times 401$ & 2,24 & 0,418 & 0,436 & 0,383 & 0.436 \\
\hline $150 \times 401$ & 2.24 & 0,865 & 0,824 & 0,757 & 0,791 \\
\hline $200 \times 401$ & 1.25 & 0.814 & 0,64 & 0,601 & 0,594 \\
\hline $100 \times 1001$ & 2.1 & 3.291 & 7.35 & 5,863 & 7,35 \\
\hline $250 \times 1001$ & 0.9 & 8,04 & 11894 & 9,781 & 9,571 \\
\hline $500 \times 1001$ & 0.5 & 12,326 & 17,154 & 14,632 & 12,841 \\
\hline $200 \times 2001$ & $1: 05$ & 47.932 & 100.156 & 86,716 & 85.54 \\
\hline $500 \times 2001$ & 0.45 & 137,08 & 187,62 & 162,4 & 156,18 \\
\hline $1000 \times 2001$ & 0.25 & 240,34 & 290,7 & 266,72 & 240,34 \\
\hline $300 \times 3001$ & 0,7 & 244,24 & 367.62 & 311,36 & 309,2 \\
\hline $750 \times 3001$ & 0.43 & 938,18 & 1117.6 & 1028.1 & 955.76 \\
\hline $400 \times 51$ & 5,88 & 0.022 & 0,02 & 0.016 & 0,02 \\
\hline $400 \times 101$ & 2,97 & 0.055 & 0,05 & 0.05 & 0,05 \\
\hline $400 \times 151$ & 1,49 & 0,214 & 0,152 & 0,151 & 0,144 \\
\hline $400 \times 201$ & 2,97 & 0,322 & 0,201 & 0,201 & 0,214 \\
\hline $1000 \times 101$ & 2,65 & 0,101 & 0,07 & 0,076 & 0,101 \\
\hline $1000 \times 251$ & 0,6 & 0,899 & 0.63 & 0,68 & 0,899 \\
\hline $1000 \times 501$ & 0.6 & 3,895 & 3.432 & 3,447 & 3,895 \\
\hline $2000 \times 201$ & 1.2 & 0,766 & 0.568 & 0,638 & 0,809 \\
\hline $2000 \times 501$ & 0.3 & 5.808 & 5.196 & 5,414 & 6,334 \\
\hline $2000 \times 1001$ & 0,53 & 37,298 & 39.996 & 37,586 & 40,07 \\
\hline $3000 \times 301$ & 1.49 & 2,096 & 1.772 & 1.948 & 3312 \\
\hline $3000 \times 751$ & 1 & 26,75 & 27,502 & 26,642 & 28.466 \\
\hline
\end{tabular}

Tabcla 6.2 - Comparaçâo entre o tempo de resolução para diferentes números de atualizações

\begin{tabular}{|c|c|c|c|c|c|}
\hline \multicolumn{6}{|c|}{ Preenchimento por atualização } \\
\hline \multirow[b]{2}{*}{ Problemas } & \multirow[b]{2}{*}{$\% \mathrm{nz}$} & \multicolumn{4}{|c|}{ Número de Atualizaçöes } \\
\hline & & 100 & $\sqrt{\mathbf{m}}$ & $3 \sqrt{\mathrm{m} / 2}$ & $0.1 \mathrm{~m}$ \\
\hline $50 \times 401$ & 4,24 & 10,393 & 10.131 & 10,118 & 15,243 \\
\hline $100 \times 401$ & 2,24 & 5,659 & 5,321 & 5,287 & 5,321 \\
\hline $150 \times 401$ & 2,24 & 5,21 & 4,626 & 4.681 & 7,014 \\
\hline $200 \times 401$ & 1,25 & 2.898 & 2.573 & 2.575 & 3,048 \\
\hline $100 \times 1001$ & 2,1 & 12,725 & 12.163 & 12,326 & $\uparrow 2,163$ \\
\hline $250 \times 1001$ & 0.9 & 5,594 & 5.225 & 5,238 & 7.328 \\
\hline $500 \times 1001$ & 0,5 & 2,98 & 2,555 & 2,607 & 4,146 \\
\hline $200 \times 2001$ & 1,05 & 12,436 & 12,132 & $12: 188$ & 18,044 \\
\hline $500 \times 2001$ & 0.45 & 5.524 & 5,168 & 5,182 & 7276 \\
\hline $1000 \times 2001$ & 0.25 & 2,94 & 2,5 & 2,514 & 2.94 \\
\hline $300 \times 3001$ & 0.7 & 12.53 & 11,878 & $1 \uparrow, 884$ & $17: 764$ \\
\hline $750 \times 3001$ & 0,43 & 7.45 & 6.91 & 6.93 & 9.27 \\
\hline \multicolumn{6}{|l|}{+2} \\
\hline $400 \times 51$ & 5,88 & 1,181 & 1.709 & 1,484 & 1,82 \\
\hline $400 \times 101$ & 2,97 & 1,41 & 1.692 & 1,61 & 2,496 \\
\hline $400 \times 151$ & 1,49 & 1,409 & 2.203 & 2.145 & 3,261 \\
\hline $400 \times 201$ & 2.97 & 1,277 & 1,59 & 1.575 & 2,54 \\
\hline $1000 \times 101$ & 2,65 & 1,945 & 1,647 & 1,572 & 1,945 \\
\hline $1000 \times 251$ & 0,6 & 1.471 & 1,714 & 1,685 & 1,471 \\
\hline $1000 \times 501$ & 0,6 & 1.724 & 1.482 & 1,483 & 1,724 \\
\hline $2000 \times 201$ & 1,2 & 1.515 & 1.808 & 1,688 & 1,741 \\
\hline $2000 \times 501$ & 0.3 & 1,522 & 1.764 & 1,752 & 2,264 \\
\hline $2000 \times 1001$ & 0.53 & 2,358 & 1.482 & 1,494 & 2,723 \\
\hline $3000 \times 301$ & 1,49 & 1.62 & 1.894 & 1.832 & 1651 \\
\hline $3000 \times 751$ & 1 & 1.818 & 2.374 & 2.322 & 3132 \\
\hline
\end{tabular}

Tabela 6.3 - Comparação entre o preenchimento atualizaçăo para diferentes números de atualizações 
Segundo a tabela 6.2. o tempo de resolução para o valor 100 foi menor na maioria dos problemas quando $n \cdots m$ e o maior quando $m \cdots n$. Para o valor $\sqrt{m}$ ocorreu exatamente o oposto do valor 100 , ou seja, $\sqrt{m}$ foi o melhor quando $m>n$ e pior quando $n>m$. Os valores $3 \mathrm{~V} m / 2$ e $0.1 m$ foram próximos em quase todos os problemas quando $n>m$ e quando $m>n$. os valores $\sqrt{m}$ e $j \sqrt{m} / 2$ é que foram próximos.

Com relação ao preenchimento por atualização, a tabela 6.3 mostra que o valor 100 fezz menos preenchimentos na maioria dos problemas para o caso em que $m=n$ e o valor $\sqrt{ } m$ lix menos preenchimentos para o caso em que $n \neq m$. Para os valores $\sqrt{m}$ e $3 \sqrt{m} / 2$ os resultados foram proximos na maioria dos problemas para os dois casos $(n>m \mathrm{e} m>n)$. () valor $0.1 \mathrm{~m}$ foi o pior em quase todos os problemas.

\begin{tabular}{|c|c|c|c|c|c|}
\hline \multicolumn{6}{|c|}{ Tempo por atualizaçäo em segundos } \\
\hline \multirow[b]{2}{*}{ Problemas } & \multirow[b]{2}{*}{$\%$ nz } & \multicolumn{4}{|c|}{ Número de Atualizaçōes } \\
\hline & & 100 & $\sqrt{\mathbf{m}}$ & $3 \sqrt{\mathrm{m}} / 2$ & $0.1 \mathrm{~m}$ \\
\hline $50 \times 401$ & 4.24 & 0 & 0 & 0 & 0 \\
\hline $100 \times 401$ & 2,24 & 0 & 0 & 0 & 0 \\
\hline $150 \times 401$ & 2,24 & 0 & 0 & 0 & 0 \\
\hline $200 \times 401$ & 1,25 & 0 & 0 & 0 & 0 \\
\hline $100 \times 1001$ & 2,1 & 0,009 & 0,012 & 0,012 & 0.012 \\
\hline $250 \times 1001$ & 0,9 & 0,011 & 0,011 & 0,011 & 0.011 \\
\hline $500 \times 1001$ & 0.5 & 0,011 & 0,01 & 0.01 & 0,01 \\
\hline $200 \times 2001$ & 1,05 & 0,108 & 0.196 & 0.188 & 0.183 \\
\hline $500 \times 2001$ & 0,45 & 0,178 & 0,204 & 0,196 & 0.206 \\
\hline $1000 \times 2001$ & 0,25 & 0,19 & 0,198 & 0.192 & 0,19 \\
\hline $300 \times 3001$ & 0,7 & 0,458 & 0,494 & 0,48 & 0.497 \\
\hline $750 \times 3001$ & 0,43 & 0.832 & 0.836 & 0.83 & 0.846 \\
\hline $400 \times 5 \overline{1}$ & 5,88 & 0 & 0 & 0 & 0 \\
\hline $400 \times 101$ & 2,97 & 0 & 0 & 0 & 0 \\
\hline $400 \times 151$ & 149 & 0 & 0 & 0 & 0 \\
\hline $400 \times 201$ & 297 & 0 & 0 & 0 & 0 \\
\hline $1000 \times 101$ & 2.65 & 0 & 0 & 0 & 0 \\
\hline $1000 \times 251$ & 0,6 & 0 & 0 & 0 & 0 \\
\hline $1000 \times 501$ & 0,6 & 0 & 0 & 0 & 0 \\
\hline $2000 \times 201$ & 1,2 & 0 & 0 & 0 & 0 \\
\hline $2000 \times 501$ & 0,3 & 0 & 0 & 0 & 0 \\
\hline $2000 \times 1001$ & 0.53 & 0.02 & 0,02 & 0.02 & 0.02 \\
\hline $3000 \times 301$ & 1,49 & 0 & 0 & 0 & 0 \\
\hline $3000 \times 751$ & 1 & 0.01 & 0,01 & 0,01 & 0.01 \\
\hline
\end{tabular}

Tabela 6.4 - Comparaçào entre o lempo atualização para diterentes números de atualizaçòes

Pela tabela 6.4. vemos que o tempo gasto por atualizaçào foi muito próximo para os valores $\sqrt{ } m, 3 \sqrt{ } m / 2$ e $0.1 m$ na maioria dos problemas no caso em que $n$ m e ligeiramente inferior ao valur 100 . Já no caso $\mathrm{cm}$ que $m \cdots n$ os resultados foram iguais para todos os problemas. Com relação ao preenchimento por decomposição de acordo com a tabela 6.5 , o valor 100 foi o melhor para a maioria dos problemas nos casos em que $n>m$ e $m>n$ e o valor $0.1 \mathrm{~m}$ foi o pior em todos os problemas na maioria dos problemas nos casos em que 
$m>n$ e $n>m$. (S valores $\sqrt{m}$ e $3 \sqrt{m} / 2$ tiveram resultados muito próximos em quase todos os problemas.

\begin{tabular}{|c|c|c|c|c|c|}
\hline \multicolumn{6}{|c|}{ Preenchimento por decomposição } \\
\hline \multirow[b]{2}{*}{ Problemas } & \multirow[b]{2}{*}{$\% n z$} & \multicolumn{4}{|c|}{ Número de Atualizações } \\
\hline & & 100 & $\sqrt[m]{m}$ & $3 \sqrt{\mathrm{m}} / 2$ & $0.1 \mathrm{~m}$ \\
\hline $50 \times 401$ & 4,24 & 0 & 0 & 0 & 0 \\
\hline $100 \times 401$ & 2,24 & 0 & 0 & 0 & 0 \\
\hline $150 \times 401$ & 2,24 & 0,45 & 1.107 & 1,249 & 0,295 \\
\hline $200 \times 401$ & 1,25 & 0.033 & 0.059 & 0,027 & 1,089 \\
\hline $100 \times 1001$ & 2,1 & 0 & 0 & 0 & 0 \\
\hline $250 \times 1001$ & 0.9 & 0 & 0.005 & 0,014 & 0,791 \\
\hline $500 \times 1001$ & 0,5 & 0.234 & 0.37 & 0,31 & 1,012 \\
\hline $200 \times 2001$ & 1.05 & 0 & 0 & 0 & 0 \\
\hline $500 \times 2001$ & 0.45 & 0,028 & 0 & 0 & 0,578 \\
\hline $1000 \times 2001$ & 0.25 & 0,362 & 0.304 & 0.382 & 0,362 \\
\hline $300 \times 3001$ & 0,7 & 0 & 0 & 0 & 0,04 \\
\hline $750 \times 3001$ & 0.43 & 5,028 & 5.77 & 5.956 & 0,086 \\
\hline \\
\hline $400 \times 51$ & 5,88 & 0.25 & 0.74 & 0,95 & 0,461 \\
\hline $400 \times 101$ & 297 & 0,7 & 1.141 & 1,25 & 1,372 \\
\hline $400 \times 151$ & 1,49 & 1,266 & 10.047 & 10,198 & 1.513 \\
\hline $400 \times 201$ & 2,97 & 1,167 & 1,508 & 1.443 & 1.872 \\
\hline $1000 \times 101$ & 2.65 & 9,066 & 1.63 & 1.59 & 9.066 \\
\hline $1000 \times 251$ & 0,6 & 3,849 & 3.216 & 3.145 & 3.849 \\
\hline $1000 \times 501$ & 0,6 & 7,95 & 4.236 & 4.389 & 7,95 \\
\hline $2000 \times 201$ & 1,2 & 3,355 & 4,102 & 4,362 & 4.466 \\
\hline $2000 \times 501$ & 0,3 & 8,616 & 8,664 & 7,858 & 8,13 \\
\hline $2000 \times 1001$ & 0,53 & 49,446 & 9,044 & 9,012 & 9.828 \\
\hline $3000 \times 301$ & 1,49 & 5,42 & 5,738 & 6,098 & 5.536 \\
\hline $3000 \times 751$ & 1 & 5,18 & 49.902 & 48.504 & 6.54 \\
\hline
\end{tabular}

Tabela 6.5 - Comparação entre o preenchimento decomposição para diferentes números de atualizaçõos

\begin{tabular}{|c|c|c|c|c|c|}
\hline \multicolumn{6}{|c|}{ Tempo por decomposiçäo em segundos } \\
\hline & \multicolumn{4}{|c|}{ Número de Atualizações } \\
\hline Problemas & $\% n z$ & 100 & $\sqrt{\mathbf{m}}$ & $3 \sqrt{\mathrm{m}} / 2$ & $0.1 \mathrm{~m}$ \\
\hline $50 \times 401$ & 4,24 & 0 & 0,012 & 0,013 & 0,012 \\
\hline $100 \times 401$ & 2,24 & 0,015 & 0,018 & 0,016 & 0.018 \\
\hline $150 \times 401$ & 2,24 & 0.017 & 0,02 & 0,02 & 0,02 \\
\hline $200 \times 401$ & 1,25 & 0.016 & 0.019 & 0.016 & 0.018 \\
\hline $100 \times 1001$ & 2,1 & 0,211 & 0.303 & 0,289 & 0.303 \\
\hline $250 \times 1001$ & 0,9 & 0,311 & 0.332 & 0.317 & 0,312 \\
\hline $500 \times 1001$ & 0.5 & 0.318 & 0.352 & 0.347 & 0,32 \\
\hline $200 \times 2001$ & 1,05 & 1.702 & 1.852 & 1.888 & 1,811 \\
\hline $500 \times 2001$ & 0,45 & 2.046 & 2,122 & 2.038 & 2,124 \\
\hline $1000 \times 2001$ & 0,25 & 2.234 & 2,29 & 2,25 & 2,234 \\
\hline $300 \times 3001$ & 0,7 & 4,338 & 5.164 & 4,958 & 5,003 \\
\hline $750 \times 3001$ & 0.43 & 6,604 & 7,004 & 6.798 & 6,613 \\
\hline $400 \times 51$ & 5,88 & 0 & 0 & 0 & 0 \\
\hline $400 \times 101$ & 2,97 & 0,001 & 0 & 0,002 & 0,001 \\
\hline $400 \times 151$ & 1,49 & 0,006 & 0,004 & 0,005 & 0,005 \\
\hline $400 \times 201$ & 2,97 & 0,002 & 0,004 & 0,007 & 0,004 \\
\hline $1000 \times 101$ & 2,65 & 0.006 & 0 & 0 & 0,006 \\
\hline $1000 \times 251$ & 0,6 & 0,031 & 0.01 & 0.01 & 0,031 \\
\hline $1000 \times 501$ & 0.6 & 0,032 & 0.033 & 0.032 & 0,032 \\
\hline $2000 \times 201$ & 1,2 & 0,008 & 0.006 & 0,004 & 0,008 \\
\hline $2000 \times 501$ & 0,3 & 0,38 & 0.034 & 0,034 & 0,031 \\
\hline $2000 \times 1001$ & 0.53 & 0,216 & 0.402 & 0.4 & 0,378 \\
\hline $3000 \times 301$ & 1.49 & 0,006 & 0.01 & 0,01 & 0,01 \\
\hline $3000 \times 751$ & 1 & 0,01 & 0,226 & 0,224 & 0,201 \\
\hline
\end{tabular}

Tahela 6.6 - Comparação entre o tempodecomposição para diferentes numeros de alualizações 
l' para linalizar, com relação ao tempo por decomposição, a tabela 6.6 mostra que o valor 100 toi mais rápido na maioria dos problemas tanto no caso em que $n>m$ quanto no caso em que $m>n$. O valor $\sqrt{m}$ loi o pior para todos os problemas quando $n>m$. Os valores $\sqrt{m}, 3 \sqrt{m} / 2$ e $0.1 m$ tiveram resultados próximos quando $m>n$.

A tabela 6.7 abaixo traz um resumo de como foi o desempenho dos quatro valores sendo do melhor para o pior desempenho olhando as colunas da esquerda para a direita. ou seja, na primeira coluna estão os valores que apresentaram melhor desempenho, depois o valor que apresentou o segundo melhor desempenho e assim sucessivamente.

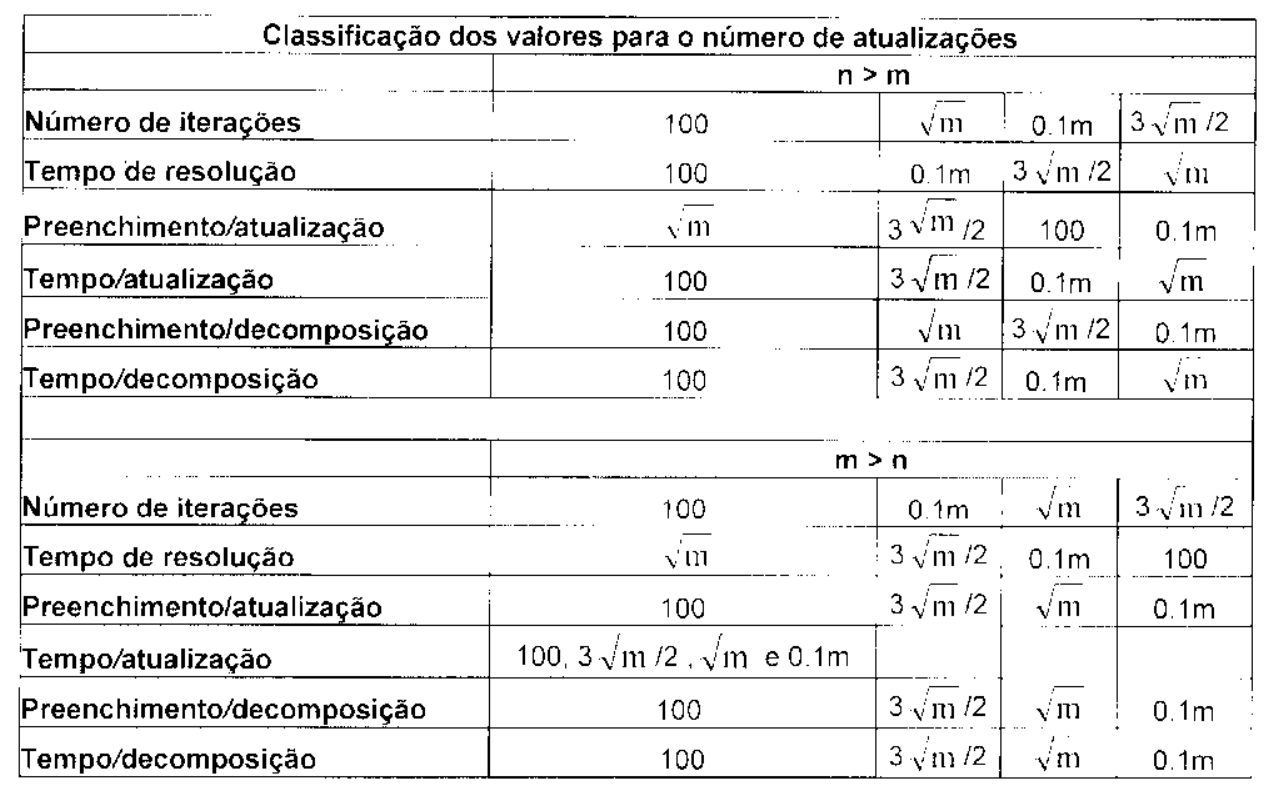

Tabela 6.7 - Comparaçâo entre alguns valores para o múmero de atualizaçōes

Observando a tabela 6.7 vemos que o valor 100 foi o melhor em quase todos itens analisados para a maioria dos problemas nos casos em que $n \cdots m$ e $m>n$.

Com relação ao número de iterações o melhor valor foi 100 para todos os problemas e o pior foi $3 \sqrt{m} / 2$. Para o tempo total de resolução o valor 100 foi melhor quando $n>m$ e o valor $\sqrt{m}$ quando $m>n$. Em se tratando do preenchimento por atualização o valor $\sqrt{m}$ foi o melhor quando $n>m$ e o valor 100 foi melhor para $m>n$. O preenchimento por decomposição foi menor para o valor 100 nos casos $\mathrm{cm}$ que $m>n$ e $n>m$ e maior em todos os problemas para o valor $0.1 \mathrm{~m}$. O tempo por atualização foi menor para o valor 100 quando $n$ ne igual para todos os valores quando $m$ n. Finalmente, o tempo por decomposição foi menor para o valor 100 tanto no caso em que $n \cdots m$ quanto no caso em que $m \cdots n$. 
Foram feitos outros testes para valores menores que $v_{m}$ e maiores que $0.1 m$ a os resultados obtidos foram piores que estes apresentados em todos os itcns analisados.

Por ter apresentado um melhor desempenho na maioria dos itens o valor 100 foi utilizado para os demais testes.

A seguir, estão os testes realizados com os quatro geradores (produção. escada, bloco angular e bloco angular em vários niveis) para poder identificar entre as combinações das heurísticas de Markowitz e Grau Mínimo com as duas variantes propostas por Reid. qual apresenta um melhor desempenho com relação a quase todos os itens analisados anteriormente para os diferentes valores do numero de atualizações que são: tempo total de resolução, preenchimento por atualização, tempo por atualização, preenchimento por decomposição e tempo por decomposição. O número de iterações foi igual para todas as combinações e pode ser visto na segunda coluna de cada tabela. Para todos os geradores foram construidos problemas com ordens muito próximas, pois exatamente igual foi impossivel devido à estrutura de cada um. Para os problemas construídos pelo gerador produção temos somente o caso $\mathrm{em}$ que $n>m$. uma ve que este gerador não constrói problemas no caso en que $m>n$.

\section{- Gerador 1: Produção}

\begin{tabular}{|c|c|c|c|c|c|c|}
\hline \multirow[b]{3}{*}{ Problemas } & \multicolumn{6}{|c|}{ Tempo Total de Resolução em segundos } \\
\hline & \multirow[b]{2}{*}{ Iteraçōes } & \multirow[b]{2}{*}{$\% n z$} & \multicolumn{2}{|c|}{ Grau Mínimo } & \multicolumn{2}{|c|}{ Markowitz } \\
\hline & & & Variante 1 & Variante 2 & Variante 1 & Variante 2 \\
\hline $60 \times 100$ & 70.8 & 3,25 & 0.04 & 0,02 & 0.042 & 0.021 \\
\hline $110 \times 200$ & 136.7 & 1,77 & 0.29 & 0,16 & 0.287 & 0.143 \\
\hline $160 \times 300$ & 195,1 & 1,22 & 0.95 & 0,56 & 0949 & 0.524 \\
\hline $220 \times 400$ & 269,4 & 0,9 & 1.98 & 0,97 & 2.071 & 0,905 \\
\hline $255 \times 500$ & 321,4 & 0,75 & 4.77 & 2.78 & 4,464 & 2.56 \\
\hline $320 \times 600$ & 393,1 & 0,62 & 6,53 & 2,94 & 6,94 & 2,785 \\
\hline $420 \times 800$ & 530,8 & 0,47 & 15.44 & 7.11 & 17,054 & 7,194 \\
\hline $505 \times 1000$ & 643,2 & 0,38 & 42.51 & 32,31 & 40.452 & 38,608 \\
\hline $620 \times 1200$ & 775 & 0,32 & 69,61 & 35,41 & 76,926 & 37,308 \\
\hline $755 \times 1500$ & 964,6 & 0,25 & 326.58 & 256.30 & 334,26 & 241,8 \\
\hline $1010 \times 2000$ & 1285,8 & 0.19 & 1119,62 & 556,38 & 1158,2 & 471,2 \\
\hline $1210 \times 2400$ & 1521,4 & 0,16 & 2124.60 & 932,04 & 2177,2 & 887,98 \\
\hline
\end{tabular}

Tabela 6.8 - Tempo total de resolução de problemas usando gerador produção 


\section{Tempo de Resolução}

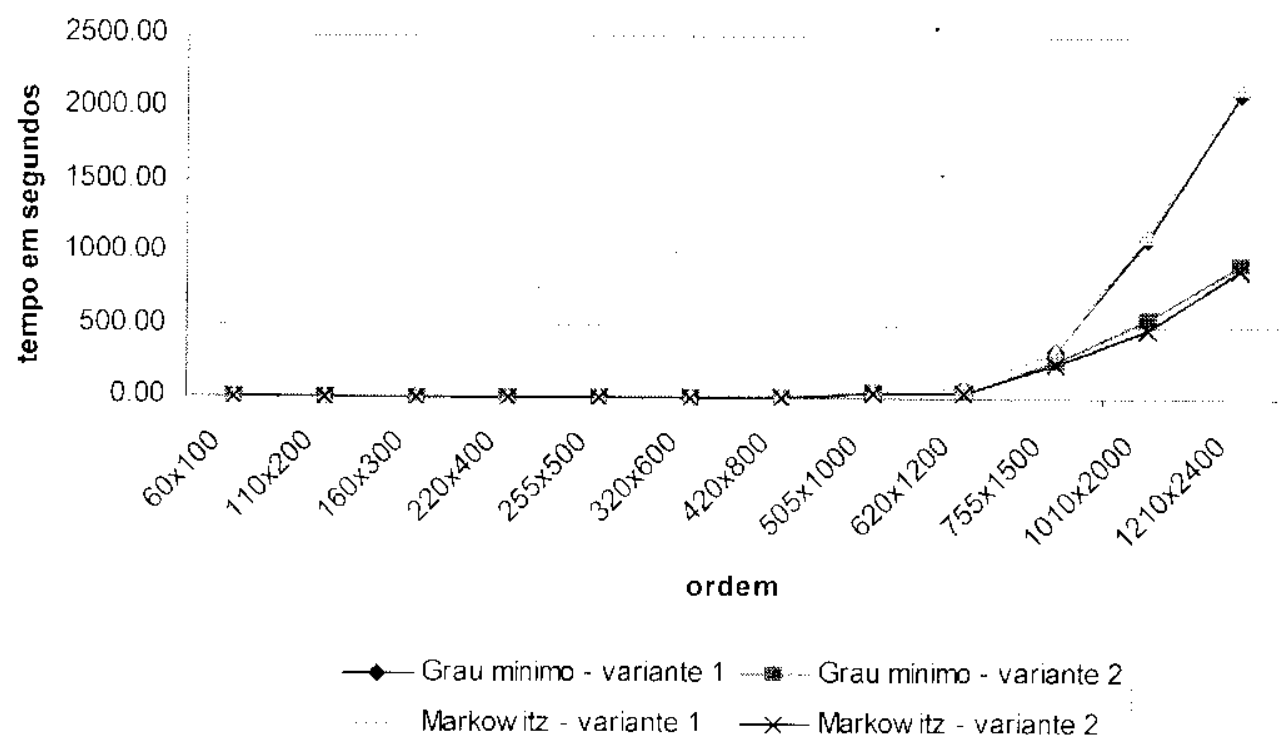

Figura 6.10 - Tempo total de resoluçào de problemas usando gerador produçâo

\begin{tabular}{|c|c|c|c|c|c|c|}
\hline \multicolumn{7}{|c|}{ Tempo por atualização em segundos } \\
\hline & & & Grau & inimo & Marl & witz \\
\hline Problemas & Iterações & $\% n z$ & Variante 1 & Variante 2 & Variante 1 & Variante 2 \\
\hline $60 \times 100$ & 70,8 & 3,25 & 0 & 0 & 0 & 0 \\
\hline $110 \times 200$ & 136,7 & 1,77 & 0 & 0 & 0 & 0 \\
\hline $160 \times 300$ & $195: 1$ & 1.22 & 0 & 0 & 0 & 0 \\
\hline $220 \times 400$ & 2694 & 0,9 & 0.007 & 0 & 0,009 & 0 \\
\hline $255 \times 500$ & 321,4 & 0.75 & 0,01 & 0,002 & 0,01 & 0 \\
\hline $320 \times 600$ & 393,1 & 0,62 & 0,01 & 0 & 0,01 & 0 \\
\hline $420 \times 800$ & 530,8 & 0,47 & 0.02 & 0,01 & 0,02 & 0,008 \\
\hline $505 \times 1000$ & 643,2 & 0,38 & 0,054 & 0,036 & 0,048 & 0,044 \\
\hline $620 \times 1200$ & 775 & 0,32 & 0,072 & 0.03 & 0,082 & 0,032 \\
\hline $755 \times 1500$ & 964,6 & 0,25 & 0,314 & 0.238 & 0,322 & 0,22 \\
\hline $1010 \times 2000$ & 1285.8 & 0,19 & 0.814 & 0.39 & 0,862 & 0,32 \\
\hline $1210 \times 2400$ & 1521.4 & 0.16 & 1.338 & 0,556 & 1,336 & 0.522 \\
\hline
\end{tabular}

Tabcla 6.9 - Tempo por alualizaçào usando gerador produçâo

De acordo com as tabelas 6.8 e 6.9. as combinações Grau Mínimo com variante 1 e Markowitz com a variante 1 tiveram resultados próximos com relação ao tempo total de resolução e ao kempo gasto por atualização. O mesmo aconteceu com as combinações Grau Mínimo com a variante 2 a Markowity com a variante 2. Estas duas últimas combinações foram mais rápidas e, apesar da pouca diferença entre elas a combinação Markowitz com a variante 2 foi um pouco melhor na maioria dos problemas, principalmente nos maiores. A diferença das duas primeiras combinações para as outras duas varia em torno de $50 \%$ em quase todos problemas. 


\begin{tabular}{|c|c|c|c|c|c|c|}
\hline \multirow[b]{3}{*}{ Problemas } & \multicolumn{5}{|c|}{ Tempo por decomposição em segundos } & \multirow{2}{*}{ witz } \\
\hline & & & \multicolumn{2}{|c|}{ Grau Mínimo } & Markowitz & \\
\hline & Iteraçöes & $\% n z$ & Variante 1 & Variante 2 & Variante 1 & Variante 2 \\
\hline $60 \times 100$ & 70,8 & 3,25 & 0 & 0 & 0 & 0 \\
\hline $110 \times 200$ & 136.7 & 1.77 & 0.002 & 0,004 & 0,007 & 0.003 \\
\hline $160 \times 300$ & 195.1 & 1.22 & 0.01 & 0,007 & 0,011 & 0,01 \\
\hline $220 \times 400$ & 269,4 & 0.9 & 0,018 & 0.016 & 0,018 & 0,018 \\
\hline $255 \times 500$ & 321,4 & 0,75 & 0,038 & 0,029 & 0,039 & 0,033 \\
\hline $320 \times 600$ & 393,1 & 0,62 & 0,041 & 0,037 & 0,048 & 0,039 \\
\hline $420 \times 800$ & 530,8 & 0,47 & 0,128 & 0.088 & 0.196 & 0.14 \\
\hline $505 \times 1000$ & 643,2 & 0.38 & 0.428 & 0,314 & 0.57 & 0.48 \\
\hline $620 \times 1200$ & 775 & 0.32 & 0.716 & 0,602 & 0.826 & 0,746 \\
\hline $755 \times 1500$ & 964,6 & 0,25 & 1,578 & 1,364 & 1,752 & 1,586 \\
\hline $1010 \times 2000$ & 1285,8 & 0,19 & 2,572 & 2,296 & 3,022 & 2,738 \\
\hline $1210 \times 2400$ & 1521,4 & 0,16 & 3,948 & 3,384 & 4,95 & 4,216 \\
\hline
\end{tabular}

Tabela 6.10 - Tempo por decomposição usando gerador produção

Pela tabela 6.10. podemos ver que com relação ao tempo gasto por decomposição, apesar da combinação Grau Minimo com a variante 2 ter sido a melhor, não houveram grandes diferenças entre todas as combinações na maioria dos problemas. Comparando as tabelas 6.9 e 6.10 vemos que o tempo por decomposição é bem maior que o tempo de atualização.

\begin{tabular}{|c|c|c|c|c|c|c|}
\hline \multirow[b]{3}{*}{ Problemas } & \multirow[b]{3}{*}{ Iteraçōes } & \multicolumn{3}{|c|}{ Preenchimento por atualização } & \multirow{2}{*}{\multicolumn{2}{|c|}{ Markowitz }} \\
\hline & & \multirow[b]{2}{*}{$\% n z$} & \multicolumn{2}{|c|}{ Grau Mínimo } & & \\
\hline & & & Variante 1 & Variante 2 & Variante 1 & Variante 2 \\
\hline $60 \times 100$ & 70,8 & 3,25 & 2,053 & $2: 014$ & 2,053 & 2.014 \\
\hline $110 \times 200$ & 136,7 & 1,77 & 4.855 & 4.895 & 2,211 & 1.961 \\
\hline $160 \times 300$ & $195: 1$ & 1.22 & 4.831 & 4.826 & 2,461 & 2.151 \\
\hline $220 \times 400$ & $269: 4$ & 0.9 & 5.339 & 5.451 & 1,968 & 1,738 \\
\hline $255 \times 500$ & $321: 4$ & 0,75 & 8.459 & 8,442 & 2,743 & 2,351 \\
\hline $320 \times 600$ & 393.1 & 0,62 & 7,293 & 7,35 & 2,024 & 1.773 \\
\hline $420 \times 800$ & 530,8 & 0,47 & 8,968 & 9.152 & 2,086 & 1,774 \\
\hline $505 \times 1000$ & 643,2 & 0,38 & 14,414 & 13,554 & 2,71 & 2,322 \\
\hline $620 \times 1200$ & 775 & 0,32 & 11,594 & 11,88 & 2,108 & 1,824 \\
\hline $755 \times 1500$ & 964,6 & 0,25 & 20.92 & 20,188 & 2,796 & 2,35 \\
\hline $1010 \times 2000$ & 1285,8 & 0,19 & 25,776 & 23,766 & 2,566 & 2,09 \\
\hline $1210 \times 2400$ & 1521,4 & 0,16 & 30,188 & 29,406 & 2,428 & 2,11 \\
\hline
\end{tabular}

Tabela 6.11 - Preenchimento por atualização usando gerador produçào 
problemas loi aumentando. Comparando ainda os resultados das tabelas 6.11 e 6.12 podemos observar que os preenchimentos fcitos por decomposição foram bem menores que os preenchimentos feitos por atualização.

Em geral:

- Melhor Combinação: Markowitz com a variante 2.

- Pior Combinação: Markowit com a variante 1.

\section{Gerador 2: Bloco Angular}

\begin{tabular}{|c|c|c|c|c|c|c|}
\hline \multirow[b]{3}{*}{ Problemas } & \multicolumn{5}{|c|}{ Tempo Total de Resolução em segundos } & \\
\hline & \multirow[b]{2}{*}{ Iterações } & \multirow[b]{2}{*}{$\% n z$} & \multicolumn{2}{|c|}{ Grau Minimo } & \multicolumn{2}{|c|}{ Markowitz } \\
\hline & & & Variante 1 & Variante 2 & Variante 1 & Variante 2 \\
\hline $60 \times 400$ & 78,7 & 5 & 0,622 & 0,388 & 0,628 & 0,389 \\
\hline $90 \times 400$ & 116,3 & 3.33 & 0,994 & 0,815 & 0,959 & 0,802 \\
\hline $158 \times 400$ & 159,6 & 2.53 & 1.502 & 1,124 & 1,331 & 1,032 \\
\hline $204 \times 400$ & 87 & 1,47 & 0,612 & 0,248 & 0,607 & 0,247 \\
\hline $120 \times 1000$ & 324.625 & 2,5 & 65,33625 & 71,315 & 29,296 & 49,826 \\
\hline $258 \times 1000$ & 1758 & 1,16 & 11.201 & 11,037 & 10,334 & 11,031 \\
\hline $504 \times 1000$ & 210,7 & 0,6 & 11.397 & 9,603 & 12,007 & 7,604 \\
\hline $220 \times 2000$ & 377,6 & 1,36 & 421.24 & 374,42 & 317,62 & 317,3 \\
\hline $508 \times 2000$ & 308,6 & 0,59 & 269.68 & 192.38 & 268.04 & 115,57 \\
\hline $1004 \times 2000$ & 395,8 & 0,3 & 305,86 & 163.26 & 347.3 & 93.806 \\
\hline $320 \times 3000$ & 448,75 & $0,94 !$ & 1164,75 & 1081,82 & 981.26 & 866,42 \\
\hline $762 \times 3000$ & 740,75 & 0,52 & 1862,5 & 1419.5 & 1514 & 1105,15 \\
\hline $402 \times 50$ & 77,3 & 4,23 & 0.026 & 0,016 & 0,025 & 0,012 \\
\hline $402 \times 100$ & 134.7 & 2,24 & 0.116 & 0,055 & 0,114 & 0,056 \\
\hline $403 \times 150$ & 176,8 & 2,23 & 0,403 & 0,199 & 0,407 & 0,204 \\
\hline $402 \times 200$ & 197.7 & 1,24 & 0,394 & 0,226 & 0,396 & 0,225 \\
\hline $1002 \times 100$ & 172,2 & 2,1 & 0.16 & 0,087 & 0.159 & 0,093 \\
\hline $1002 \times 250$ & 336 & 0,9 & 1,415 & 0.73 & 1.422 & 0,726 \\
\hline $1002 \times 500$ & 418,2 & 0,5 & 5,717 & 2.376 & 5.81 & 2271 \\
\hline $2002 \times 200$ & 357,4 & 1,05 & 1,186 & 0,714 & 1,132 & 0.706 \\
\hline $2002 \times 500$ & 662 & 0,45 & 10,244 & 4,04 & 10,336 & 4,248 \\
\hline $2002 \times 1000$ & 856,4 & $0,25:$ & 57,568 & 27,212 & 59,152 & 26.156667 \\
\hline $3002 \times 300$ & 573,6 & 0.7 & 4,036 & 2,302 & 3,88 & 2,25 \\
\hline $3003 \times 750$ & 1269 & 0,43 & 72,314 & 34,602 & 62,44 & 39,74 \\
\hline
\end{tabular}

Tabela 6.13 - Tempo total de resoluçăo de problemas usando gerador bloco angular 


\section{Preenchimento por atualização}

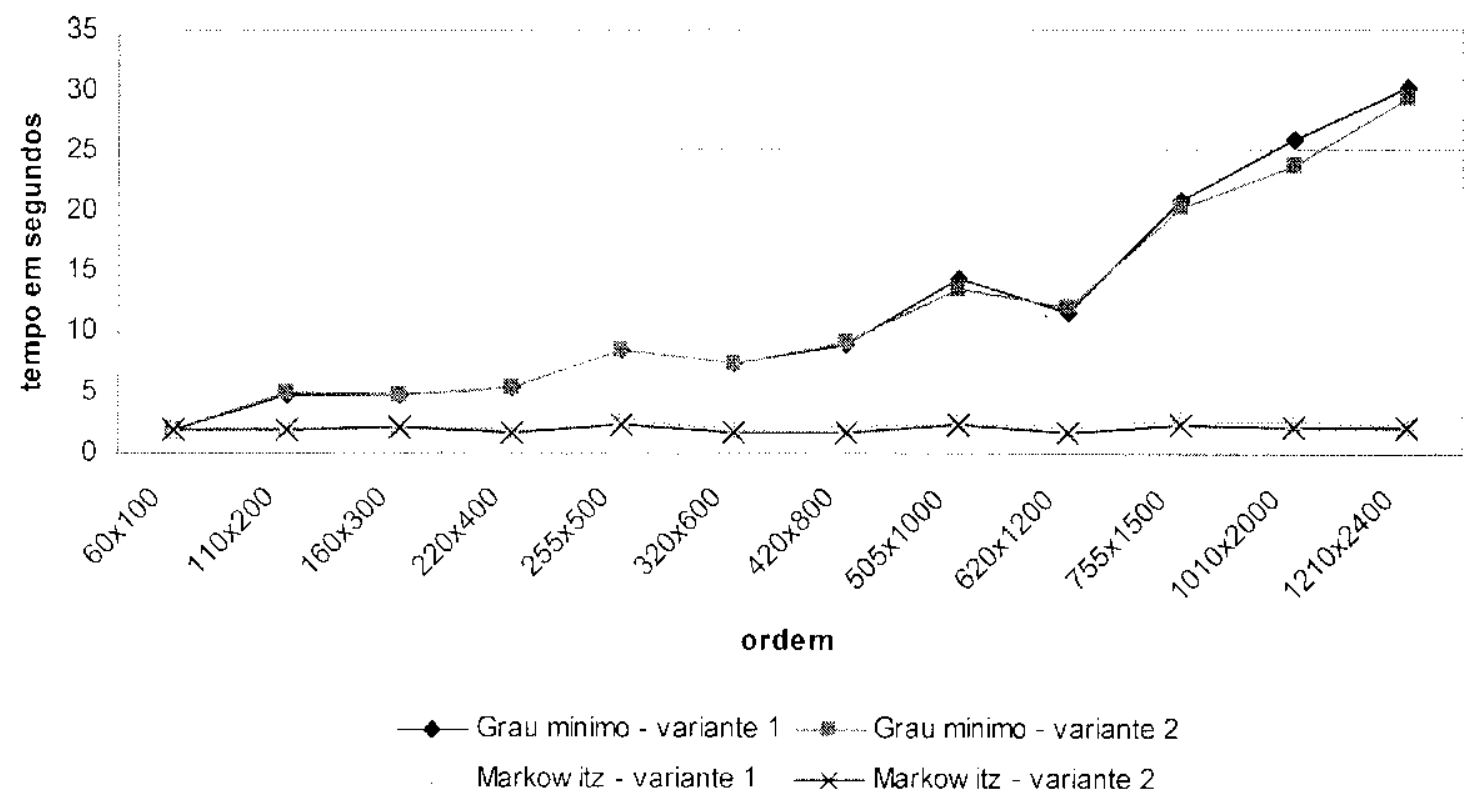

Figura 6.11 - Preenchimento por atualizaçào usando gerador produção

\begin{tabular}{|c|c|c|c|c|c|c|}
\hline \multicolumn{7}{|c|}{ Preenchimento por decomposição } \\
\hline \multirow[b]{2}{*}{ Problemas } & \multirow[b]{2}{*}{ Iteraçöes } & \multirow[b]{2}{*}{$\%$ nz } & \multicolumn{2}{|c|}{ Grau Minimo } & \multicolumn{2}{|c|}{ Markowitz } \\
\hline & & & Variante 1 & Variante 2 & Variante 1 & Variante 2 \\
\hline $60 \times 100$ & 70,8 & 3,25 & 0 & 0 & 0 & 0 \\
\hline $110 \times 200$ & 136,7 & 1,77 & 1,95 & 1,95 & 0,2 & 0,2 \\
\hline $160 \times 300$ & 195,1 & 1,22 & 10,867 & 10,867 & 0,7 & 0,7 \\
\hline $220 \times 400$ & 269,4 & 0,9 & 8,468 & 8.468 & 0.732 & 0.732 \\
\hline $255 \times 500$ & 321,4 & 0,75 & 19,605 & 11,85 & 0,3 & 0,2 \\
\hline $320 \times 600$ & 393,1 & 0,62 & 4,735 & 4,735 & 0,58 & 0.58 \\
\hline $420 \times 800$ & 530,8 & 0,47 & 8.034 & 5,234 & 0,4 & 0,4 \\
\hline $505 \times 1000$ & 643,2 & 0,38 & 0 & 0 & 0 & 0,228 \\
\hline $620 \times 1200$ & 775 & 0.32 & 4.098 & 4.098 & 0,188 & 0,388 \\
\hline $755 \times 1500$ & 964,6 & 0,25 & 33.88 & 26.98 & 0,44 & 0,24 \\
\hline $1010 \times 2000$ & 1285,8 & 0,19 & 73,408 & 60,398 & 0,426 & 0,634 \\
\hline $1210 \times 2400$ & 1521,4 & 0,16 & 105,448 & 54,26 & 0,954 & 1,166 \\
\hline
\end{tabular}

Tabela 6.12 - Preenchimento por decomposição usando gerador produção

Analisando as tabelas 6.11 e 6.12 vemos que as combinações Markowitz com a variante 1 e Markowitz com a variante 2 foram muito próximas em relação ao preenchimento por atualização c por decomposição, apesar da segunda combinação ter sido um pouco melhor que a primeira. Os preenchimentos por atualização feitos nas combinações Markowitz com a variante 1 e Markowitz com a variante 2 foram estáveis para todas as ordens dos problemas. diferente do que ocorreu com as combinações Grau Mínimo com a variante 1 e (jrau Mínimo con a variante 2, onde houve um aumento nos preenchimentos conforme a ordem dos 


\section{Tempo de Resolução $(n>m)$}

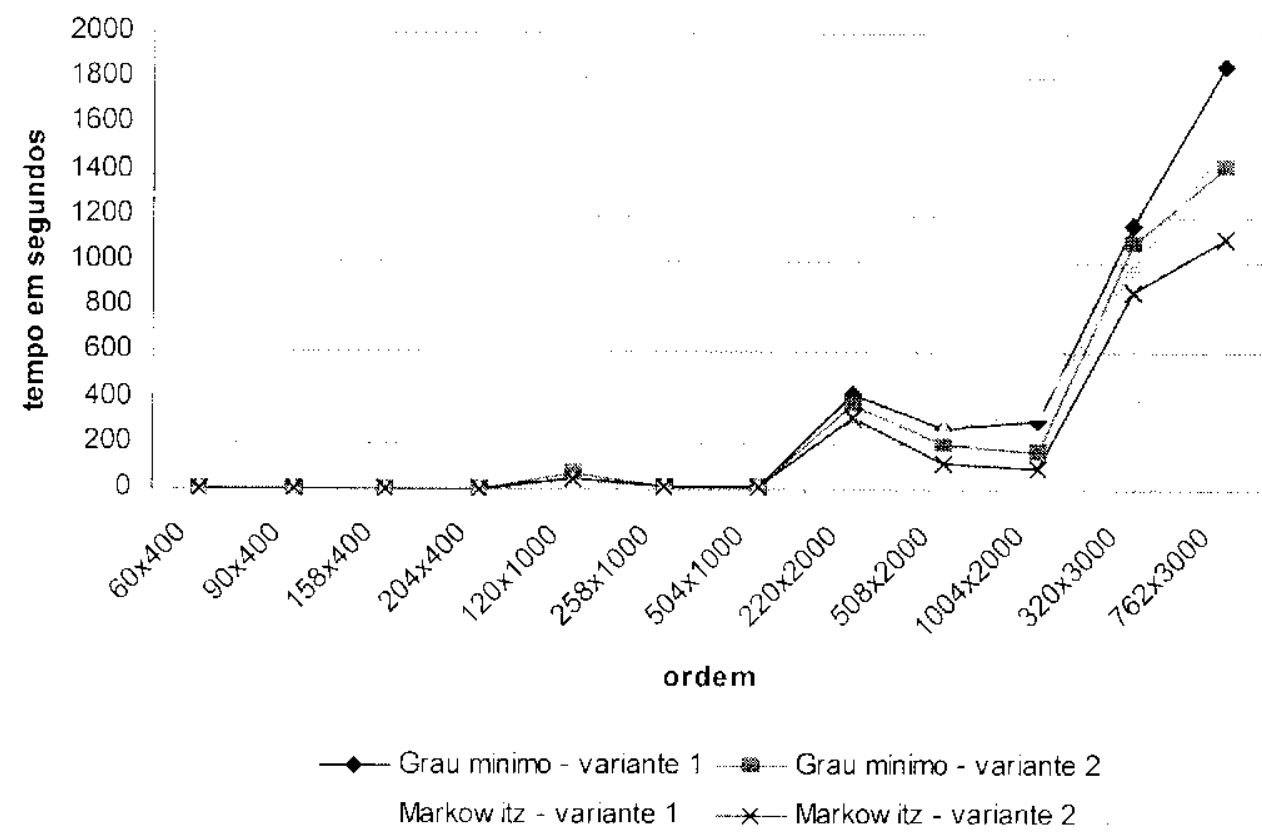

Figura 6.12 - lempo total de resoluçào de problemas usando gerador bloco angular

\section{Tempo de Resolução $(m>n)$}

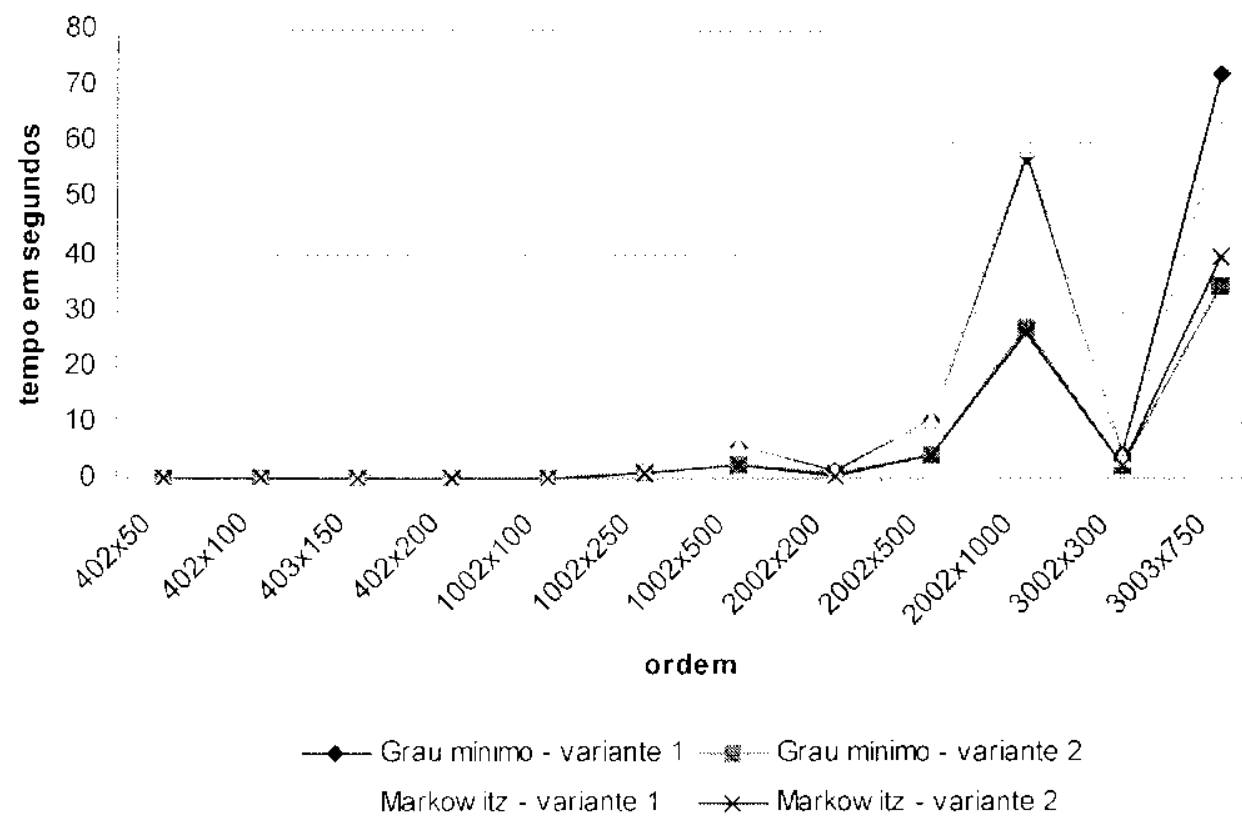

Figura 6.13 - Tempo total de resolução de problemas usando gerador bloco angular 


\begin{tabular}{|c|c|c|c|c|c|c|}
\hline \multicolumn{7}{|c|}{ Tempo por atualizaçäo em segundos } \\
\hline & & & \multicolumn{2}{|c|}{ Grau Minimo } & \multicolumn{2}{|c|}{ Markowitz } \\
\hline Problemas & Iteraçöes & $\% \mathrm{nz}$ & Variante 1 & Variante 2 & Variante 1 & Variante 2 \\
\hline $60 \times 400$ & 78,7 & 5 & 0,006 & 0 & 0,008 & 0 \\
\hline $90 \times 400$ & 116,3 & 3,33 & 0,005 & 0 & 0,005 & 0 \\
\hline $158 \times 400$ & 159,6 & 2,53 & 0,009 & 0,001 & 0,006 & 0 \\
\hline $204 \times 400$ & 87 & 1,47 & 0.004 & 0 & 0,005 & 0 \\
\hline $120 \times 1000$ & 324,625 & 2.5 & 0,1725 & 0,191 & 0,068 & 0,128 \\
\hline $258 \times 1000$ & 175,8 & 1,16 & 0.049 & 0,049 & 0,045 & 0,049 \\
\hline $504 \times 1000$ & 210,7 & 0,6 & 0.041 & 0,032 & 0,041 & 0,021 \\
\hline $220 \times 2000$ & 377,6 & 1,36 & 1.054 & 0,924 & 0.78 & 0,776 \\
\hline $508 \times 2000$ & 308,6 & 0,59 & 0.83 & 0.574 & 0,818 & 0,318 \\
\hline $1004 \times 2000$ & 3958 & 0,3 & 0,732 & 0.37 & 0.828 & 0.184 \\
\hline $320 \times 3000$ & 448,75 & 0.94 & 2,465 & $2: 232$ & 1,976 & 1,76 \\
\hline $762 \times 3000$ & 740,75 & 0.52 & 2,385 & 1,7725 & 1,895 & 1,3325 \\
\hline $402 \times 50$ & 77.3 & 4,23 & 0 & 0 & 0 & 0 \\
\hline $402 \times 100$ & 134,7 & 2.24 & 0 & 0 & 0 & 0 \\
\hline $403 \times 150$ & 176,8 & 2,23 & 0 & 0 & 0 & 0 \\
\hline $402 \times 200$ & 197.7 & 1,24 & 0 & 0 & 0 & 0 \\
\hline $1002 \times 100$ & 172.2 & 2,1 & 0 & 0 & 0 & 0 \\
\hline $1002 \times 250$ & 336 & 0,9 & 0 & 0 & 0 & 0 \\
\hline $1002 \times 500$ & 418,2 & 0.5 & 0,01 & 0 & 0,01 & 0 \\
\hline $2002 \times 200$ & 357,4 & 1,05 & 0 & 0 & 0 & 0 \\
\hline $2002 \times 500$ & 662 & 0,45 & 0.01 & 0 & 0,01 & 0 \\
\hline $2002 \times 1000$ & 856.4 & 0,25 & 0,054 & 0.02 & 0,056 & 0,02 \\
\hline $3002 \times 300$ & 573.6 & 0,7 & 0 & 0 & 0 & 0 \\
\hline $3003 \times 750$ & 1269 & 0.43 & 0,044 & 0.018 & 0,036 & 0.02 \\
\hline
\end{tabular}

Tabela 6.14 - Tempo por atualizaçào usando gerador bloco angular

\begin{tabular}{|c|c|c|c|c|c|c|}
\hline \multicolumn{7}{|c|}{ Tempo por decomposição em segundos } \\
\hline \multirow[b]{2}{*}{ Problemas } & \multirow[b]{2}{*}{ Iteraçöes } & \multirow[b]{2}{*}{$\% \mathrm{nz}$} & \multicolumn{2}{|c|}{ Grau Mínimo } & \multicolumn{2}{|c|}{ Markowitz } \\
\hline & & & Variante 1 & Variante 2 & Variante 1 & Variante 2 \\
\hline $60 \times 400$ & 78,7 & 5 & 0,004 & 0,004 & 0 & 0 \\
\hline $90 \times 400$ & 116,3 & 3,33 & 0.021 & 0.016 & 0,016 & 0.016 \\
\hline $158 \times 400$ & 159.6 & 2,53 & 0,023 & 0,022 & 0,022 & 0.021 \\
\hline $204 \times 400$ & 87 & 1.47 & 0,004 & 0,004 & 0 & 0 \\
\hline $120 \times 1000$ & 324,625 & 2,5 & 0,81625 & 0.77 & 0,737 & 0,702 \\
\hline $258 \times 1000$ & 175,8 & 1,16 & 0.318 & 0,247 & 0,389 & 0,356 \\
\hline $504 \times 1000$ & 210,7 & 0,6 & 0.333 & 0,336 & 0.454 & 0,403 \\
\hline $220 \times 2000$ & 377,6 & 1,36 & 3.524 & 3,392 & 3,674 & 3,578 \\
\hline $508 \times 2000$ & 308.6 & 0,59 & 2,546 & 2,312 & 3,092 & 2,938 \\
\hline $1004 \times 2000$ & 395,8 & 0,3 & 2,552 & 2,318 & 3,386 & 3.168 \\
\hline $320 \times 3000$ & 448,75 & 0,94 & 8,9075 & $8: 214$ & 9,062 & 8.638 \\
\hline $762 \times 3000$ & $740: 75$ & 0,52 & 10,2925 & 9,455 & 9,955 & 9,5875 \\
\hline \\
\hline $402 \times 50$ & 77.3 & 4,23 & 0 & 0 & 0 & 0 \\
\hline $402 \times 100$ & 134.7 & 2,24 & 0,003 & 0,001 & 0,004 & 0.001 \\
\hline $403 \times 150$ & 176.8 & 2.23 & 0,008 & 0,005 & 0.006 & 0,007 \\
\hline $402 \times 200$ & 197,7 & 1,24 & 0,006 & 0,003 & 0,006 & 0,006 \\
\hline $1002 \times 100$ & 172,2 & 2.1 & 0,001 & 0,001 & 0,002 & 0,001 \\
\hline $1002 \times 250$ & 336 & 0,9 & 0.007 & 0.007 & 0,011 & 0,01 \\
\hline $1002 \times 500$ & 418,2 & 0,5 & 0,036 & 0,03 & 0.044 & 0,039 \\
\hline $2002 \times 200$ & 357,4 & 1,05 & 0,002 & 0,002 & 0.008 & 0.008 \\
\hline $2002 \times 500$ & 662 & 0,45 & 0,034 & 0,028 & 0,066 & 0,058 \\
\hline $2002 \times 1000$ & 856,4 & 0,25 & 0,454 & 0,394 & 0,584 & 0.5233333 \\
\hline $3002 \times 300$ & 573.6 & 0.7 & 0.01 & 0.01 & 0,028 & 0,028 \\
\hline $3003 \times 750$ & 1269 & 0.43 & 0,256 & 0.198 & 0.442 & 0.39 \\
\hline
\end{tabular}

Tabela 6.15 - I impo por decomposiçăo usando gerador bloco angular 
De acordo com as tabelas 6.13 e 6.14. para a maioria dos problemas nos dois casos em que $n$ m e $m$ o tempo total de resolução e o tempo por atualização das combinações Grau Mínimo com a variante 1 e Markowitz com a variante 1 são próximos. O mesmo acontece com as combinações Grau Mínimo com a variante 2 e Markowitz com a variante 2. Fistas duas combinações apresentaram melhor desempenho e apesar da pequena diferença entre elas, principalmente no caso em que $m>n$ e nos problemas pequenos do caso $n \therefore m$, a combinação Markowit\% com a variante 2 foi a melhor.

Com relação an tempo gasto por decomposição, de acordo com a tabela 6.15, houve uma troca nos pares de combinações. pois na maioria dos problemas. considerando os dois casos em que $n>m$ e $m>n$, as combinações ( Irau Mínimo com a variante 1 e Grau Mínimo com a variante 2 apresentaram resultados próximos e melhores que as combinações Markuwitz com a variante 1 e Markowitz com a variante 2, exceto para os problemas pequenos no caso $\mathrm{cm}$ que $n>m$, onde a melhor combinação foi Markowitz com a variante 2.

Podemos observar também das tabelas 6.13.6.14 e 6.15 que o tempo total de resolução c o tempo gasto por atualização e por decomposição foram bem menores para todos os problemas no caso em que $m>n$ do que no caso em que $n>m$.

\begin{tabular}{|c|c|c|c|c|c|c|}
\hline \multicolumn{7}{|c|}{ Preenchimento por atualizaçäo } \\
\hline \multirow[b]{2}{*}{ Problemas } & \multirow[b]{2}{*}{ Iteraçōes } & \multirow[b]{2}{*}{$\% \mathrm{nz}$} & \multicolumn{2}{|c|}{ Grau Mínimo } & \multicolumn{2}{|c|}{ Markowitz } \\
\hline & & & Variante 1 & Variante 2 & Variante 1 & Variante 2 \\
\hline $60 \times 400$ & 78.7 & 5 & 10,983 & 10,688 & 10,983 & 10,688 \\
\hline $90 \times 400$ & 116,3 & 3,33 & 14,925 & 13,272 & 10,265 & 8.585 \\
\hline $158 \times 400$ & 159.6 & 2,53 & 17.286 & 15,308 & 9,621 & 7,274 \\
\hline $204 \times 400$ & 87 & 1,47 & 7,453 & 6,887 & 7,453 & 6,887 \\
\hline $120 \times 1000$ & 324,625 & 2,5 & 58,9425 & 61,533 & 16,689 & 13,767 \\
\hline $258 \times 1000$ & 175.8 & 1,16 & 23,603 & 24,656 & 11,718 & 10,564 \\
\hline $504 \times 1000$ & 210,7 & 0,6 & 11,397 & 15.45 & 7,909 & 7,359 \\
\hline $220 \times 2000$ & 377,6 & 1,36 & 89,942 & 86,714 & 19,294 & 15.514 \\
\hline $508 \times 2000$ & 308,6 & 0.59 & 43.75 & 43,236 & 13,208 & 12,014 \\
\hline $1004 \times 2000$ & 395,8 & 0,3 & 29,536 & 29,404 & 8,238 & 7,866 \\
\hline $320 \times 3000$ & 448.75 & 0,94 & 112,255 & 118,986 & 20.63 & 17,16 \\
\hline $762 \times 3000$ & 740.75 & 0,52 & 89,66 & 87,145 & 15,645 & 12,41 \\
\hline $402 \times 50$ & 77.3 & 4,23 & 1.852 & 0,746 & 1.852 & 0.756 \\
\hline $402 \times 100$ & 134,7 & 2,24 & 4.188 & 2,813 & 3.38 & 1,797 \\
\hline $403 \times 150$ & 176,8 & 2,23 & 4,079 & 3,295 & 3,063 & 2,06 \\
\hline $402 \times 200$ & 197.7 & 1,24 & 7.378 & 4.791 & 6,692 & 3.092 \\
\hline $1002 \times 100$ & 172.2 & 2,1 & 3,052 & 1.684 & 2,516 & 1,148 \\
\hline $1002 \times 250$ & 336 & 0,9 & 5.46 & 4.861 & 3,092 & 2,191 \\
\hline $1002 \times 500$ & 418.2 & 0.5 & 7.257 & 6.888 & 3,13 & 2,822 \\
\hline $2002 \times 200$ & 357.4 & 1.05 & 4.864 & 4,372 & 2,242 & 1,296 \\
\hline $2002 \times 500$ & 662 & 0,45 & 8.306 & 8.266 & 2,98 & 2,488 \\
\hline $2002 \times 1000$ & 856,4 & 0,25 & 13.366 & 13,238 & 3,552 & 3,0066667 \\
\hline $3002 \times 300$ & 573,6 & 0,7 & 6,218 & 5,432 & 2,322 & 0,932 \\
\hline $3003 \times 750$ & 1269 & 0,43 & 18,224 & 16,774 & 4,786 & 3,068 \\
\hline
\end{tabular}

Tabela 6.16 - Precenchimento por anualizaçào usando gerador bloco angular 
Preenchimento por atualização $(n>m)$

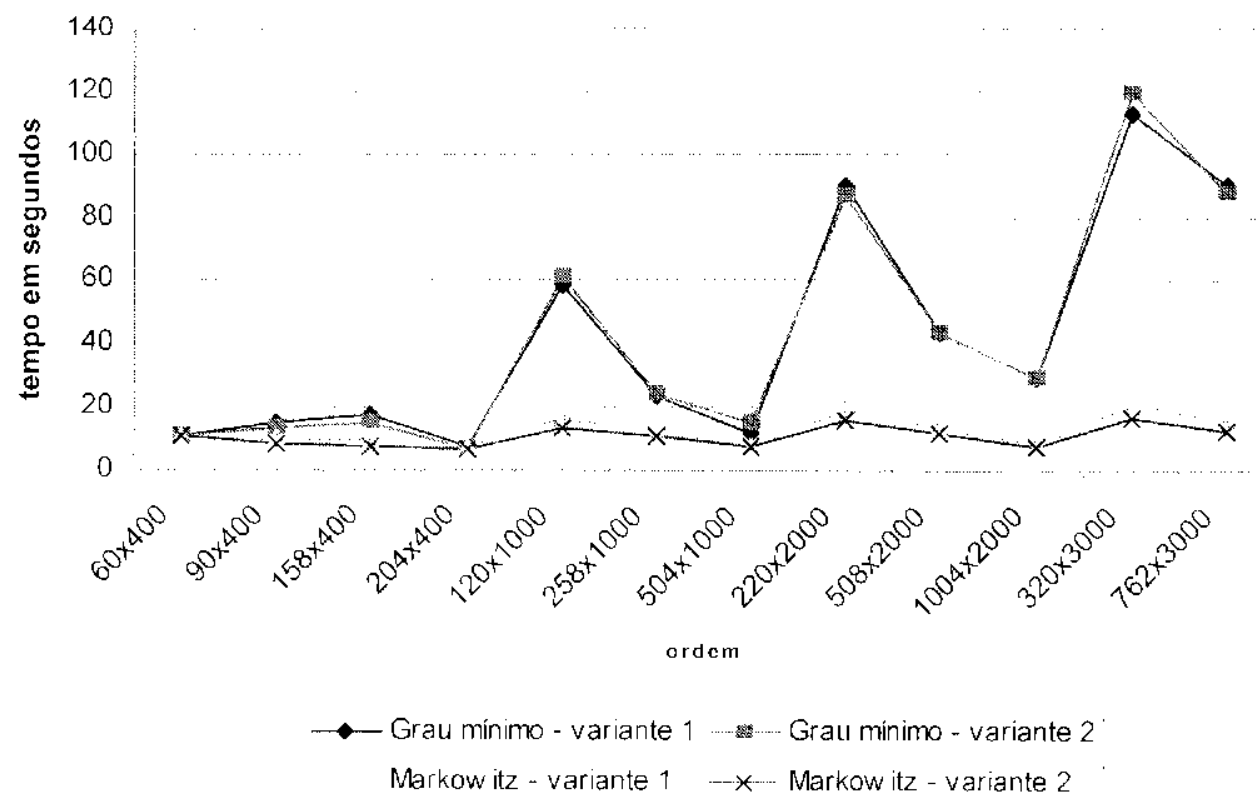

Figura 6.14 - Preenchimento por atualização usando gerador bloco angular

\section{Preenchimento por atualização $(m>n)$}

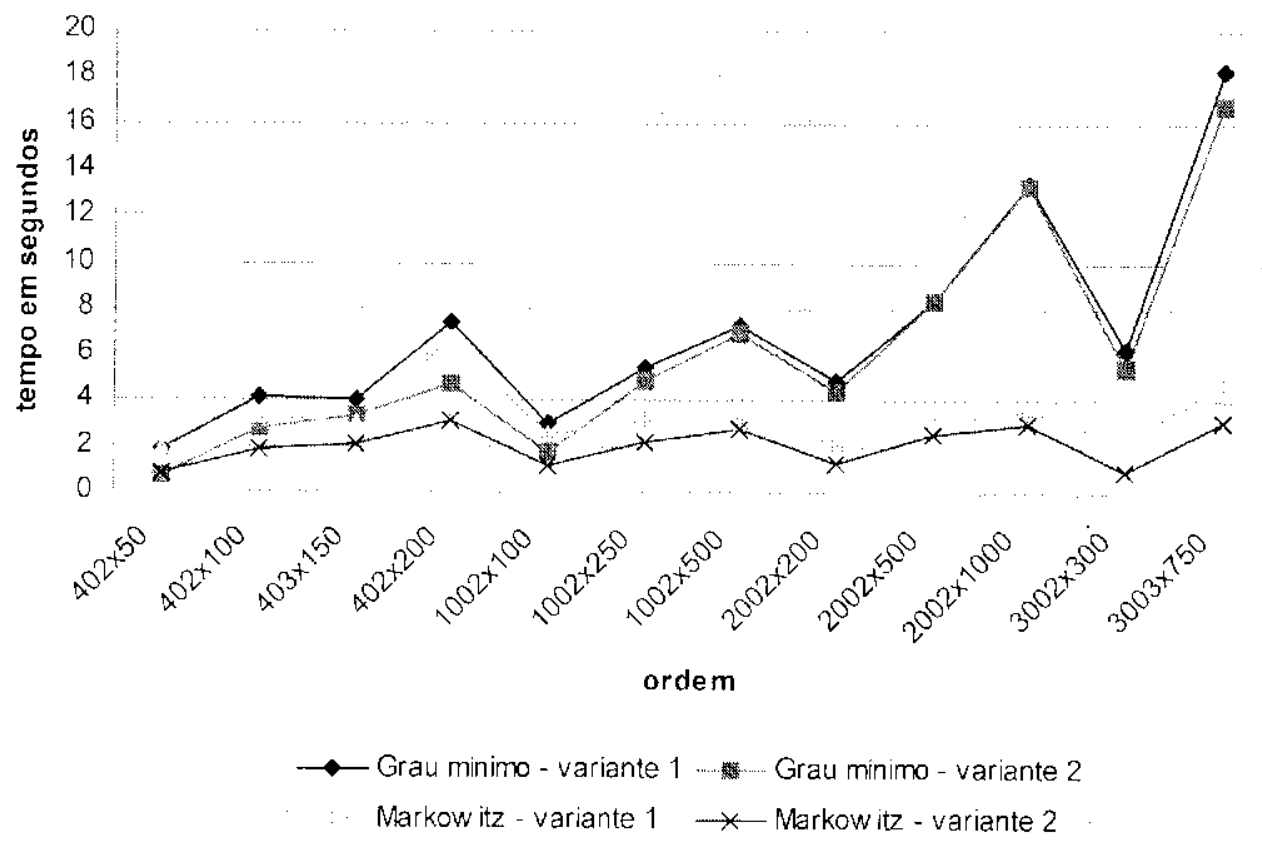

Figura 6.15 - Preenchimento por atualização usando gerador bloco angular 


\begin{tabular}{|c|c|c|c|c|c|c|}
\hline \multirow{2}{*}{ - } & \multicolumn{5}{|c|}{ Preenchimento por decomposição } & \\
\hline & & & \multicolumn{2}{|c|}{ Grau Minimo } & \multicolumn{2}{|c|}{ Markowitz } \\
\hline Problemas & Iteraçōes & $\% \mathrm{nz}$ & Variante 1 & Variante 2 & Variante 1 & Variante 2 \\
\hline $60 \times 400$ & 78,7 & 5 & 0 & 0 & 0 & 0 \\
\hline $90 \times 400$ & 116,3 & 3,33 & 147,9 & 147,9 & 6,75 & 6,75 \\
\hline $158 \times 400$ & 159,6 & 2,53 & 202,5 & 202,5 & 15,617 & 15,617 \\
\hline $204 \times 400$ & 87 & 1,47 & 0 & 0 & 0 & 0 \\
\hline $120 \times 1000$ & 324,625 & 2,5 & 1187.075 & 1182,38 & 38,8 & 41,779 \\
\hline $258 \times 1000$ & 175,8 & 1,16 & 121,45 & 103.7 & 2,65 & 2,55 \\
\hline $504 \times 1000$ & 210,7 & 0,6 & 2,3 & 17,033 & 0 & 0 \\
\hline $220 \times 2000$ & 377,6 & 1.36 & 1536.2 & 1503 & 30,24 & 29,04 \\
\hline $508 \times 2000$ & 308,6 & 0.59 & 160,994 & 160,994 & $3: 134$ & 3,134 \\
\hline $1004 \times 2000$ & 395,8 & 0.3 & 77,6 & 77.6 & 2,28 & 2,28 \\
\hline $320 \times 3000$ & 448,75 & 0,94 & 1817,75 & 1828,4 & 34,374 & 31,64 \\
\hline $762 \times 3000$ & 740,75 & 0,52 & 2001 & 1845,25 & 58,7375 & 53,935 \\
\hline $402 \times 50$ & 77,3 & 4,23 & 0 & 0 & 0 & 0 \\
\hline $402 \times 100$ & 134,7 & 2,24 & 17.4 & 17,35 & 11.75 & 12 \\
\hline $403 \times 150$ & 176,8 & 2.23 & 18.3 & 18,3 & 4,5 & 5.267 \\
\hline $402 \times 200$ & 197,7 & 1.24 & 40,034 & 40.034 & 21.75 & 20,833 \\
\hline $1002 \times 100$ & 172,2 & 2.1 & 10 & 10 & 7,05 & 6,617 \\
\hline $1002 \times 250$ & 336 & 0,9 & 41.975 & 41,975 & 26,6 & 25,375 \\
\hline $1002 \times 500$ & 418,2 & 0,5 & 52,37 & 52,37 & 14.98 & 14,78 \\
\hline $2002 \times 200$ & 357,4 & 1,05 & 39.6 & 34,6 & 32,45 & 33,6 \\
\hline $2002 \times 500$ & 662 & 0.45 & 113.432 & 106,898 & 61,218 & 55,45 \\
\hline $2002 \times 1000$ & 856.4 & 0,25 & 161.5 & 161.48 & 39,58 & 47,173333 \\
\hline $3002 \times 300$ & 573,6 & 0,7 & 70,786 & 70,786 & 53,37 & 69,528 \\
\hline $3003 \times 750$ & 1269 & 0.43 & 384.64 & 388,68 & 260,6 & 265,84 \\
\hline
\end{tabular}

Tabela 6.17 - Preenchimento por decomposiçăo usando gerador bloco angular

Analisando a tabela 6.16 , vemos que, em relação ao preenchimento por atualização, as combinações Markowitz com a variante 1 e Markowit/ con a variante 2 apresentam resultados proximos e melhores para a maioria dos problemas no caso em que $n>m$ e para os problemas maiores no caso em que $m>n$, havendo uma troca das combinações para os problemas menores no caso em que $m \sim n$ onde desta ve $\%$ as combinações (Grau Mínimo com a variante 2 e Markowity com a variante 2 tiveram menor preenchimento. Mas em todos os problemas testados, a combinação Markowitz com a variante 2 foi a melhor. De acordo com a tabela 6.17. o preenchimento por decomposição foi melhor para as combinações Markowitz com a variante 1 e Markowitz com a variante 2, sendo que a primeira foi um pouco mais rápida na maioria dos problemas. Os preenchimentos feitos por atualização e por decomposição usando a heurística de (jrau Mínimo foram maiores no caso $\mathrm{cm}$ que $n \% m$ do que no caso em que $m>n$. Para a heuristica de Markowit\% os preenchimentos feitos por decomposição foram menores no caso em que $n>m$ e maiores no caso em que $m \ngtr n$.

Iim geral:

- Melhor Combinação: Markowitz com a variante 2.

- Pior Combinação: Grau Mínimo com a variante 1. 


\begin{tabular}{|c|c|c|c|c|c|c|}
\hline \multirow[b]{3}{*}{ Problemas } & \multicolumn{6}{|c|}{ Tempo de Resolução em segundos } \\
\hline & \multirow{3}{*}{\multicolumn{2}{|c|}{ Iteraçôes }} & \multicolumn{2}{|c|}{ Grau Minimo } & \multicolumn{2}{|c|}{ Markowitz } \\
\hline & & & Variante 1 & Variante 2 & Variante 1 & Variante 2 \\
\hline $50 \times 401$ & 70,5 & & 0.514 & 0,176 & 0.511 & 0,171 \\
\hline $100 \times 401$ & 122,3 & 2,24 & 0,935 & 0,418 & 0,978 & 0,516 \\
\hline $150 \times 401$ & 180,1 & 2,24 & 1,556 & 0.865 & 1,766 & 1,029 \\
\hline $200 \times 401$ & 211,3 & 1,25 & 1,537 & 0,814 & 1,698 & 0,859 \\
\hline $100 \times 1001$ & 148,2 & 2,1 & 7,183 & 3,291 & 10,058 & 11.87 \\
\hline $250 \times 1001$ & 307.6 & 0,9 & 14,025 & 8,04 & 20,535 & 16,264 \\
\hline $500 \times 1001$ & 519,4 & 0.5 & 23,052 & 12.326 & 32,245 & 21,183 \\
\hline $200 \times 2001$ & 300.4 & 1.05 & 147.58 & 47,932 & 244.1 & 150,04 \\
\hline $500 \times 2001$ & 617 & 0.45 & 318,06 & 137.08 & 537.56 & 243.3 \\
\hline $1000 \times 2001$ & 1050.6 & 0.25 & 544,22 & 240,34 & 950,4 & 354,38 \\
\hline $300 \times 3001$ & 447.2 & 0.7 & 690,6 & 244,24 & 979 & 600,86 \\
\hline $750 \times 3001$ & 1008 & 0,43 & 1826,2 & 938,18 & 2746,2 & 1877,2 \\
\hline $400 \times 51$ & 94,3 & 5.88 & 0.029 & 0.022 & 0,028 & 0,02 \\
\hline $400 \times 101$ & 160,6 & 2,97 & 0.114 & 0,055 & 0.12 & 0,056 \\
\hline $400 \times 151$ & 222,8 & 1,49 & 0.361 & 0,214 & 0,394 & 0.225 \\
\hline $400 \times 201$ & 253 & 2,97 & 0.544 & 0,322 & 0.596 & 0.332 \\
\hline $1000 \times 101$ & 196 & 2,65 & 0.173 & 0,101 & 0.177 & 0,098 \\
\hline $1000 \times 251$ & 401,5 & 0,6 & 1,452 & 0,899 & 1.595 & 0.948 \\
\hline $1000 \times 501$ & 618,5 & 0,6 & 7,204 & 3,895 & 8.833 & 4,498 \\
\hline $2000 \times 201$ & 403,8 & 1,2 & 1,124 & 0,766 & 1,19 & 0,796 \\
\hline $2000 \times 501$ & 797,6 & 0,3 & 9,6 & 5,808 & 11,448 & 6,63 \\
\hline $2000 \times 1001$ & 1248.8 & 0,53 & 58,856 & 37.298 & 79,968 & 55,456 \\
\hline $3000 \times 301$ & 582.2 & 1.49 & 3,132 & 2,096 & 3,412 & 2,228 \\
\hline $3000 \times 751$ & 1245,8 & 1 & 40,16 & 26.75 & 52,028 & 34,364 \\
\hline
\end{tabular}

Tahela 6.18 - Timpo total de resolução de problemas usando getador escada

\section{Tempo de Resolução (n > m)}

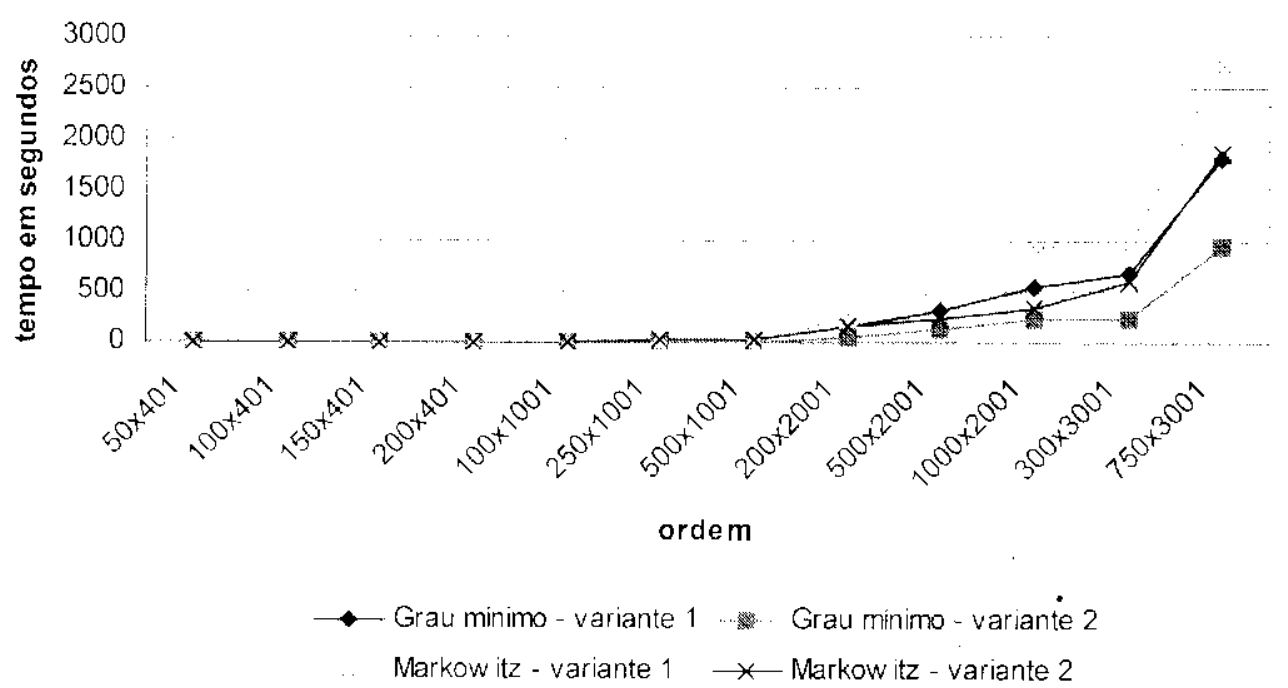

Figura 6.16 - Tempo total de resolução de problemas usando gerador escada 
Tempo de Resolução $(m>n)$

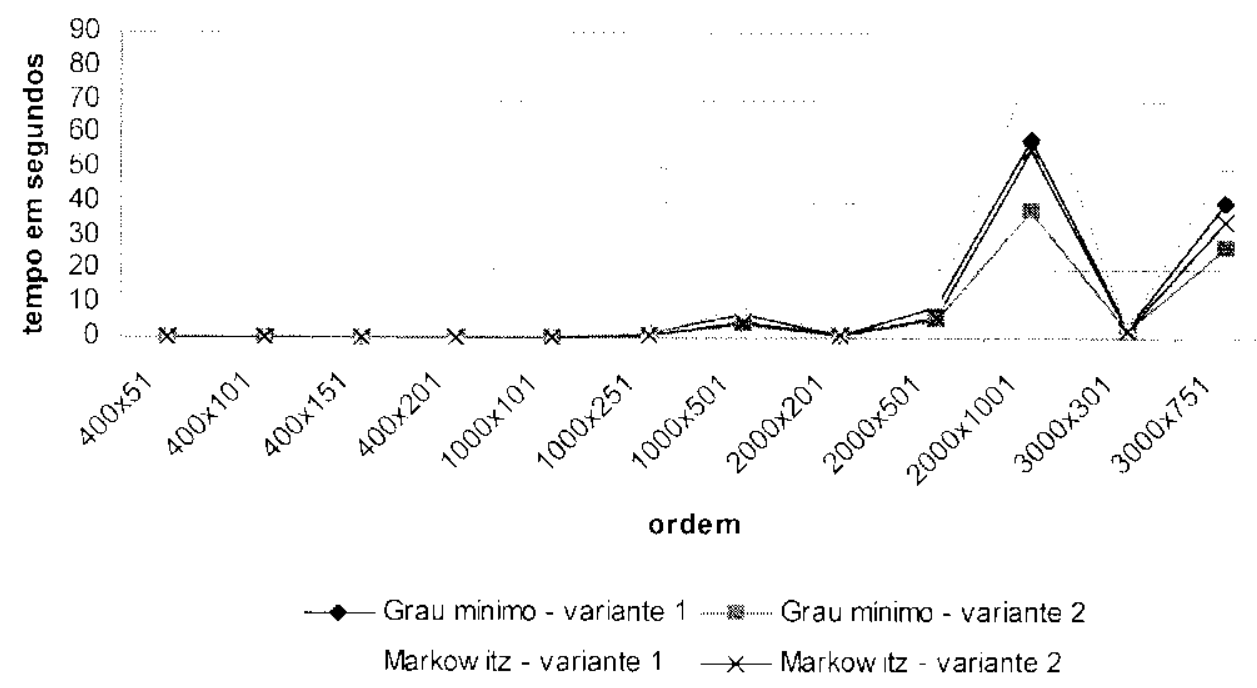

Figura 6.17 . Tempo total de resoluçăo de problemas usando gerador escada

\begin{tabular}{|c|c|c|c|c|c|c|}
\hline \multicolumn{7}{|c|}{ Tempo por atualização em segundos } \\
\hline \multirow[b]{2}{*}{ Problemas } & \multirow[b]{2}{*}{ Iterações } & \multirow[b]{2}{*}{$\% n z$} & \multicolumn{2}{|c|}{ Grau Minimo } & \multicolumn{2}{|c|}{ Markowitz } \\
\hline & & & Variante 1 & Variante 2 & Variante 1 & Variante 2 \\
\hline $50 \times 401$ & 70.5 & 4,24 & 0.007 & 0 & 0.004 & 0 \\
\hline $100 \times 401$ & 122,3 & 2,24 & 0,005 & 0 & 0,006 & 0 \\
\hline $150 \times 401$ & 180,1 & 2,24 & 0,009 & 0 & 0,01 & 0 \\
\hline $200 \times 401$ & 211.3 & 1,25 & 0,003 & 0 & 0.01 & 0 \\
\hline $100 \times 1001$ & 148.2 & 2,1 & 0,035 & 0.009 & 0.053 & 0,064 \\
\hline $250 \times 1001$ & 307,6 & 0.9 & 0.031 & 0,011 & 0.051 & 0,038 \\
\hline $500 \times 1001$ & 519,4 & 0.5 & 0.031 & 0.011 & 0.049 & 0,029 \\
\hline $200 \times 2001$ & 300,4 & 1.05 & 0.446 & $0: 108$ & 0.76 & 0.444 \\
\hline $500 \times 2001$ & 617 & 0,45 & 0.476 & 0,178 & 0,83 & 0,346 \\
\hline $1000 \times 2001$ & 1050,6 & 0,25 & 0.482 & 0,19 & 0,864 & 0,294 \\
\hline $300 \times 3001$ & 447,2 & 0,7 & 1,464 & 0,458 & 2,092 & 1,234 \\
\hline $750 \times 3001$ & 1008 & 0,43 & 1.726 & 0.832 & 2,616 & 1,746 \\
\hline $400 \times 51$ & 94,3 & 5,88 & 0 & 0 & 0 & 0 \\
\hline $400 \times 101$ & 160,6 & 2,97 & 0 & 0 & 0 & 0 \\
\hline $400 \times 151$ & 222,8 & 1,49 & 0 & 0 & 0 & 0 \\
\hline $400 \times 201$ & 253 & 2,97 & 0 & 0 & 0 & 0 \\
\hline $1000 \times 101$ & 196 & 2,65 & 0 & 0 & 0 & 0 \\
\hline $1000 \times 251$ & 401,5 & 0,6 & 0 & 0 & 0 & 0 \\
\hline $1000 \times 501$ & 618.5 & 0,6 & 0,01 & 0 & 0,01 & 0 \\
\hline $2000 \times 201$ & 403.8 & 1,2 & 0 & 0 & 0 & 0 \\
\hline $2000 \times 501$ & $797: 6$ & 0,3 & 0,01 & 0 & 0.01 & 0 \\
\hline $2000 \times 1001$ & 1248.8 & 0,53 & 0,038 & 0.02 & 0.05 & 0,03 \\
\hline $3000 \times 301$ & 582,2 & 1.49 & 0 & 0 & 0 & 0 \\
\hline $3000 \times 751$ & 12458 & 1 & 0,022 & 0.01 & 0.03 & 0,02 \\
\hline
\end{tabular}

Tabela 6.19 - Tempo por atualizaçào nsilndo gerador escuada 


\begin{tabular}{|c|c|c|c|c|c|c|}
\hline \multirow[b]{3}{*}{ Problemas } & \multicolumn{6}{|c|}{ Tempo por decomposição em segundos } \\
\hline & \multirow[b]{2}{*}{ Iteraçöes } & \multirow[b]{2}{*}{$\% n z$} & \multicolumn{2}{|c|}{ Grau Mínimo } & \multicolumn{2}{|c|}{ Markowitz } \\
\hline & & & Variante 1 & Variante 2 & Variante 1 & Variante 2 \\
\hline $50 \times 401$ & 70,5 & 4,24 & 0,002 & .0 & 0,002 & 0 \\
\hline $100 \times 401$ & 122,3 & 2,24 & 0,015 & 0,015 & 0,02 & 0,02 \\
\hline $150 \times 401$ & 180,1 & 2,24 & 0,019 & $0: 017$ & 0,02 & 0,02 \\
\hline $200 \times 401$ & 211,3 & 1,25 & 0,016 & 0,016 & 0,023 & 0,02 \\
\hline $100 \times 1001$ & 148,2 & 2,1 & 0.254 & 0211 & 0,386 & 0,334 \\
\hline $250 \times 1001$ & 307,6 & 0,9 & 0,345 & 0,311 & 0,5 & 0,44 \\
\hline $500 \times 1001$ & 519,4 & 0,5 & 0.38 & 0,318 & 0,519 & 0,454 \\
\hline $200 \times 2001$ & 300,4 & 1,05 & 1,814 & 1,702 & 2,578 & 2,456 \\
\hline $500 \times 2001$ & 617 & 0,45 & 2,17 & 2,046 & 2,87 & 2,71 \\
\hline $1000 \times 2001$ & 1050,6 & 0,25 & 2,276 & 2,234 & 3,036 & 2,83 \\
\hline $300 \times 3001$ & 447,2 & 0.7 & 4.48 & 4,338 & 6,552 & 6,376 \\
\hline $750 \times 3001$ & 1008 & 0,43 & 6,782 & 6,604 & 9,634 & 9,188 \\
\hline $400 \times 51$ & 94.3 & 5,88 & 0 & 0 & 0 & 0 \\
\hline $400 \times 101$ & 160.6 & 2.97 & 0,004 & 0,001 & 0,003 & 0.001 \\
\hline $400 \times 151$ & 222.8 & 1.49 & 0,007 & 0,006 & 0.009 & 0.008 \\
\hline $400 \times 201$ & 253 & 2.97 & 0,007 & 0006 & 0,009 & 0,011 \\
\hline $1000 \times 101$ & 196 & 2,65 & 0,001 & 0,002 & 0,002 & $\cdot 0,005$ \\
\hline $1000 \times 251$ & 401,5 & 0,6 & 0.007 & 0,008 & 0,017 & 0,018 \\
\hline $1000 \times 501$ & 618,5 & 0,6 & 0,037 & 0,031 & 0,068 & 0,059 \\
\hline $2000 \times 201$ & 403,8 & 1,2 & 0,004 & 0,006 & 0,012 & 0,014 \\
\hline $2000 \times 501$ & 797,6 & 0,3 & 0,038 & 0,032 & 0,086 & 0,08 \\
\hline $2000 \times 1001$ & 1248,8 & 0,53 & 0,448 & 0.38 & 0.7 & 0,628 \\
\hline $3000 \times 301$ & 582,2 & 1,49 & 0,012 & 0.01 & 0,028 & 0,024 \\
\hline $3000 \times 751$ & 1245,8 & 1 & 0,262 & 0,216 & 0,566 & 0.516 \\
\hline
\end{tabular}

Tabela 6.20 - Tempo por decomposição usando gerador escada

De acordo com as tabelas 6.18 e 6.19 , para a maioria dos problemas nos dois casos em que $n>m$ e $m>n$ o tempo total de resolução e o tempo por atualização das combinações (irau Mínimo com a variante 2 e Markowit\% com a variante 2 apresentaram melhor desempenho sendo que a primeira combinação foi a melhor entre as duas.

Com relação ao tempo por decomposição, de acordo com a tabela 6.20, houve uma troca nos pares de combinações na maioria dos problemas considerando os casos em que $n>m$ e $m$ m. pois as combinações Grau Mínimo com a variante 1 e Grau Mínimo com a variante 2 apresentaram resultados próximos e melhores que as combinações Markowitz com a variante 1 e Markowitz com a variante 2. As diferenças no tempo gasto por decomposição entre todas combinações. nos casos onde $n>m \mathrm{~cm}>n$, foram menores para os problemas pequenos.

Podemos observar pelas tabelas 6.18, 6.19 e 6.20 que o tempo total de resolução, o tempo por atualização e o tempo por decomposição foram menores no caso em que $m>n$ para lodos os problemas. 


\begin{tabular}{|c|c|c|c|c|c|c|}
\hline \multirow[b]{3}{*}{ Problemas } & \multicolumn{4}{|c|}{ Preenchimento por atualização } & & \\
\hline & & & Grau & ínimo & \multicolumn{2}{|c|}{ Markowitz } \\
\hline & Iterações & $\% n z$ & Variante 1 & Variante 2 & Variante 1 & Variante 2 \\
\hline $50 \times 401$ & 70,5 & 4,24 & 10.139 & 10,393 & 10.139 & 10,393 \\
\hline $100 \times 401$ & 122,3 & 2,24 & 7,071 & 7,2 & 5,424 & 5,659 \\
\hline $150 \times 401$ & 180,1 & 2,24 & 7,2 & 7,362 & 4,978 & 5,21 \\
\hline $200 \times 401$ & 211,3 & 1,25 & 4,177 & 4,315 & 2.645 & 2,898 \\
\hline $100 \times 1001$ & 148.2 & 2,1 & 18,375 & 18.475 & 12463 & 12,725 \\
\hline $250 \times 1001$ & 307.6 & 0,9 & 8.488 & $8: 567$ & 5,357 & 5.594 \\
\hline $500 \times 1001$ & 519.4 & 0.5 & 4.871 & 4,941 & 2,67 & 2,98 \\
\hline $200 \times 2001$ & 300,4 & 1,05 & 20,12 & 20,182 & 12,152 & 12,436 \\
\hline $500 \times 2001$ & 617 & 0.45 & 9,192 & 9,242 & 5,21 & 5.524 \\
\hline $1000 \times 2001$ & 1050,6 & 0,25 & 5,174 & 5.226 & 2,578 & 2,94 \\
\hline $300 \times 3001$ & 447,2 & 0.7 & 20,558 & 20,592 & 12,276 & 12.53 \\
\hline $750 \times 3001$ & 1008 & 0,43 & 13,302 & 13.358 & 7.076 & 7,45 \\
\hline $400 \times 51$ & 94,3 & 5.88 & 1.181 & 1,234 & 1,181 & 1,234 \\
\hline $400 \times 101$ & 160,6 & 2,97 & 1.583 & 1,839 & 1,41 & 1,617 \\
\hline $400 \times 151$ & 222,8 & 1,49 & 2,534 & 2,868 & 1,945 & 2.262 \\
\hline $400 \times 201$ & 253 & 2,97 & 2,189 & 2,389 & 1,409 & 1.622 \\
\hline $1000 \times 101$ & 196 & $2,65^{\circ}$ & 1,389 & 1.742 & 1.277 & 1.553 \\
\hline $1000 \times 251$ & 401,5 & 0,6 & 2,303 & 2.571 & 1.515 & 1,767 \\
\hline $1000 \times 501$ & 618.5 & 0.6 & 2.71 & 2.849 & 1471 & 1,717 \\
\hline $2000 \times 201$ & 403,8 & 1,2 & 2,088 & 2,378 & 1,62 & 1,916 \\
\hline $2000 \times 501$ & 797.6 & 0,3 & 2,626 & 2,804 & 1,724 & 1,978 \\
\hline $2000 \times 1001$ & 1248,8 & 0,53 & 2,942 & 3,034 & 1,522 & 1,794 \\
\hline $3000 \times 301$ & 582,2 & 1,49 & 2,37 & 2,572 & 1,818 & 2,048 \\
\hline $3000 \times 751$ & 1245,8 & 1 & 3,814 & 3,998 & 2,358 & 2,688 \\
\hline
\end{tabular}

Tabcla 6.21 - Preenchimento por atualização usando gerador escada

\section{Preenchimento por atualização $(n>m)$}

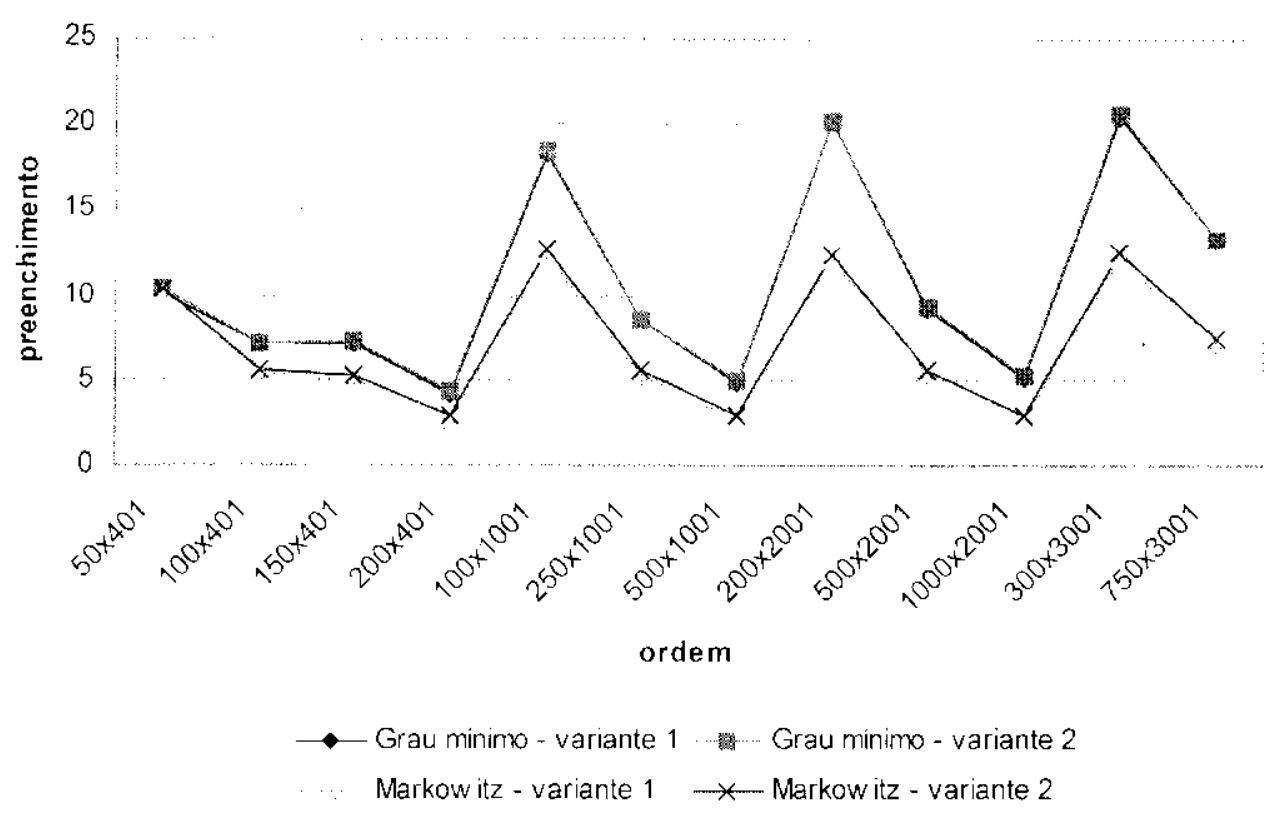

Figura 6.18 - Preenchimento por atualização usando grerador escada 


\section{Preenchimento por atualização $(m>n)$}

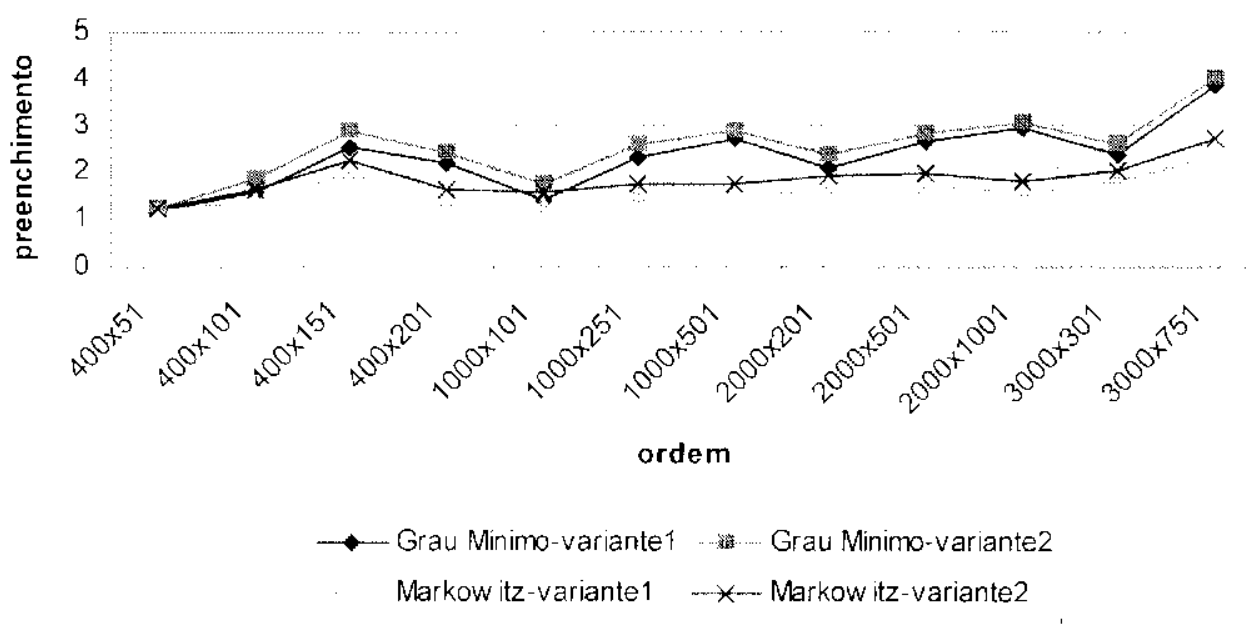

Figura 6.19 - Preenchimento por atualização usando gerador escada

\begin{tabular}{|c|c|c|c|c|c|c|}
\hline \multirow[b]{3}{*}{ Problemas } & \multicolumn{4}{|c|}{ Preenchimento por decomposiçã } & \multirow{2}{*}{\multicolumn{2}{|c|}{ Markowitz }} \\
\hline & & & \multicolumn{2}{|c|}{ Grau Minimo } & & \\
\hline & Iteraçōes & $\% n z$ & Variante 1 & Variante 2 & Variante 1 & Variante 2 \\
\hline $50 \times 401$ & 70,5 & 4,24 & 0 & 0 & 0 & 0 \\
\hline $100 \times 401$ & 122,3 & 2,24 & 0,3 & 0.3 & 0 & 0 \\
\hline $150 \times 401$ & 180,1 & 2,24 & 0.1 & 0,1 & 0,45 & 0.45 \\
\hline $200 \times 401$ & 211,3 & 1,25 & 0,865 & 0.865 & 0,033 & 0,033 \\
\hline $100 \times 1001$ & 148.2 & 2,1 & $-0,2$ & $-0,2$ & 0 & 0 \\
\hline $250 \times 1001$ & 307.6 & 0,9 & 0,608 & 0,608 & 0 & 0 \\
\hline $500 \times 1001$ & 519.4 & 0,5 & 2,6 & 2,6 & 0,234 & 0,234 \\
\hline $200 \times 2001$ & 300,4 & 1.05 & 0.25 & 0,25 & 0 & 0 \\
\hline $500 \times 2001$ & 617 & 0.45 & 0.686 & 0,686 & 0,028 & 0,028 \\
\hline $1000 \times 2001$ & 1050,6 & 0,25 & 4.69 & 4,69 & 0,362 & 0,362 \\
\hline $300 \times 3001$ & 447.2 & 0,7 & 0.12 & 0,12 & 0 & 0 \\
\hline $750 \times 3001$ & 1008 & 0,43 & 0,06 & 0.06 & 5,028 & 5.028 \\
\hline $400 \times 51$ & 94,3 & 5,88 & 0,25 & 0,25 & 0,25 & 0.25 \\
\hline $400 \times 101$ & 160,6 & 2,97 & 0,6 & 0,6 & 0.7 & 0.7 \\
\hline $400 \times 151$ & 222.8 & 1.49 & 1.602 & 1,602 & 9,066 & 9.066 \\
\hline $400 \times 201$ & 253 & 2.97 & 1.332 & 1.332 & 1,266 & 1,266 \\
\hline $1000 \times 101$ & 196 & 2.65 & 1.151 & 1,151 & 1,167 & 1,167 \\
\hline $1000 \times 251$ & 401,5 & 0,6 & 3.55 & 3.55 & 3,355 & 3,355 \\
\hline $1000 \times 501$ & 618,5 & 0.6 & 4,453 & 4,453 & 3.849 & 3.849 \\
\hline $2000 \times 201$ & 403,8 & 1,2 & 5.02 & 5,02 & 5.42 & 5.42 \\
\hline $2000 \times 501$ & 797,6 & 0,3 & 8,092 & 8,092 & 7.95 & 795 \\
\hline $2000 \times 1001$ & 1248,8 & 0,53 & 8.23 & 8,23 & 8,616 & 8,616 \\
\hline $3000 \times 301$ & 582,2 & 1,49 & 5,414 & 5.414 & 5,18 & 5,18 \\
\hline $3000 \times 751$ & 1245.8 & 1. & 6,754 & 6,754 & 49,446 & 49,446 \\
\hline
\end{tabular}

Tabela 6.22 - Preenchimento por decomposição usando gerador escada

Analisando a tabela 6.21. vemos que em relação ao preenchimento por atualização. as combinações Markowitz con a variante 1 e Markowitz com a variante 2 apresentaram resultados mais próximos e melhores para a maioria dos problemas nos casos em que $n \cdots m$ e $m$ n. mas entre estas duas combinaçôes, em todos os problenas testados, a combinação Markowit\% com a variante 1 foi a melhor. I: de acordo com a tabela 6.22, para o preenchimento por decomposição, as combinações Markowity com a variante l e Markowitz. 
com a variante 2 apresentaram resultados idênticos e melhores para a maioria dos problemas no caso em que $n>m$. Para o caso em que $m \times n$, as combinações (jrau Minimo com a variante 1 e Grau Mínimo com a variante 2 apresentaram resultados idênticos e melhores para a maioria dos problemas. Os preenchimentos feitos tanto na atualização quanto na decomposição no caso $\mathrm{cm}$ que $n \times m$ foram maiores que no caso em que $m n$ para quase todos os problemas.

Em geral:

- Melhor Combinação: Grau Mínimo com a variante 2.

- Pior Combinação: Markowitz com a variante 1.

\section{Gerador 4: Bloco angular em vários níveis}

\begin{tabular}{|c|c|c|c|c|c|c|}
\hline \multicolumn{7}{|c|}{ Tempo Total de Resolução em segundos } \\
\hline \multirow[b]{2}{*}{ Problemas } & \multirow[b]{2}{*}{ Iteraçöes } & \multirow[b]{2}{*}{$\% \mathrm{nz}$} & \multicolumn{2}{|c|}{ Grau Mínimo } & \multicolumn{2}{|c|}{ Markowitz } \\
\hline & & & Variante 1 & Variante 2 & Variante 1 & Variante 2 \\
\hline $110 \times 400$ & 140,5 & 1,76 & 1.525 & 1,129 & 1.397 & 1.188 \\
\hline $160 \times 400$ & 129,2 & 1,66 & 0.892 & 0,737 & 0,849 & 0,687 \\
\hline $191 \times 400$ & $134, \uparrow$ & 1,6 & 0,88 & 0,737 & 0,87 & 0,662 \\
\hline $241 \times 400$ & 119,8 & 1,87 & 0.817 & 0.559 & 0,798 & 0,61 \\
\hline $215 \times 1000$ & 258,7 & 1,8 & 25.796 & 28,535 & 14,217 & 23,552 \\
\hline $457 \times 1008$ & 310,1 & 1,62 & 34,855 & 33.91 & 18,427 & 22,18 \\
\hline $883 \times 1000$ & 509,6 & 1.36 & 31,669 & 30,126 & 24,393 & 17,898 \\
\hline $255 \times 2000$ & 424.8 & 1.88 & 425,34 & 351,58 & 384,92 & 332,16 \\
\hline $420 \times 2000$ & 663,8 & 0,76 & 540,82 & $413: 2$ & 543,82 & 456,82 \\
\hline $840 \times 2000$ & 687,2 & 0,72 & 556,14 & 550,82 & 516,32 & 429,48 \\
\hline $580 \times 3000$ & 792,4 & 0,76 & 1651.4 & 1337,6 & 1533 & 1188,6 \\
\hline $860 \times 3000$ & 751,6 & 1,12 & 1478,4 & 1019,78 & 1439,2 & 1098,5 \\
\hline $409 \times 78$ & 55,2 & 1,65 & 0,024 & 0,02 & 0,024 & 0,012 \\
\hline $426 \times 120$ & 82,6 & 1,55 & 0.062 & 0,052 & 0,058 & 0,052 \\
\hline $431 \times 150$ & 105.8 & 1,49 & 0,13 & 0,106 & 0,132 & 0,106 \\
\hline $456 \times 300$ & 204,4 & 1.29 & 0,858 & 0,646 & 0,874 & 0,562 \\
\hline $1031 \times 150$ & 101,6 & 1.72 & 0,132 & 0.11 & 0,132 & 0,11 \\
\hline $1077 \times 378$ & 253,6 & 1.49 & 1,512 & 1,196 & 1,63 & 1,194 \\
\hline $1131 \times 750$ & 591,2 & 1,3 & 12,16 & 11,026 & 13,274 & 10,17 \\
\hline $2035 \times 300$ & 215 & 1,28 & 1,16 & 0,82 & 1,024 & 0.794 \\
\hline $2065 \times 750$ & 531,8 & 0,91 & 13.238 & 10,61 & 14.688 & 12.168 \\
\hline $2115 \times 1500$ & 1182,8 & 0,68 & 195 & 311,86 & 354,6 & 353,94 \\
\hline $3045 \times 450$ & 306,8 & 1,28 & 3.038 & 2,342 & $3: 172$ & 2,42 \\
\hline $3065 \times 750$ & 506,2 & 1,07 & 12.674 & 11,108 & 14,586 & 10,874 \\
\hline
\end{tabular}

Tabela 6.23 - Tempo total de resolução de problemas usando gerador bloco angular em vários níveis 


\section{Tempo de Resolução (n > m)}

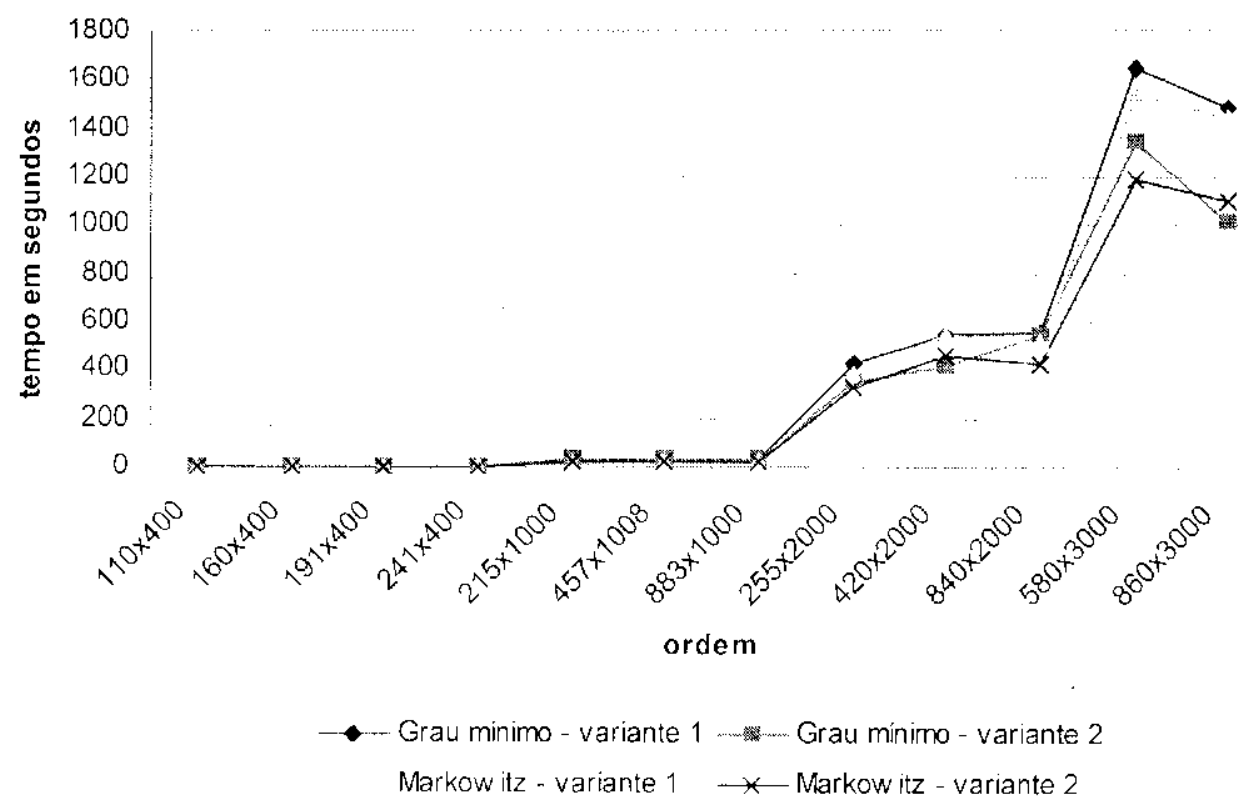

Figura 6.20 - Tempo por decomposição usando gerador bloco angular em vários niveis

\section{Tempo de Resolução $(m>n)$}

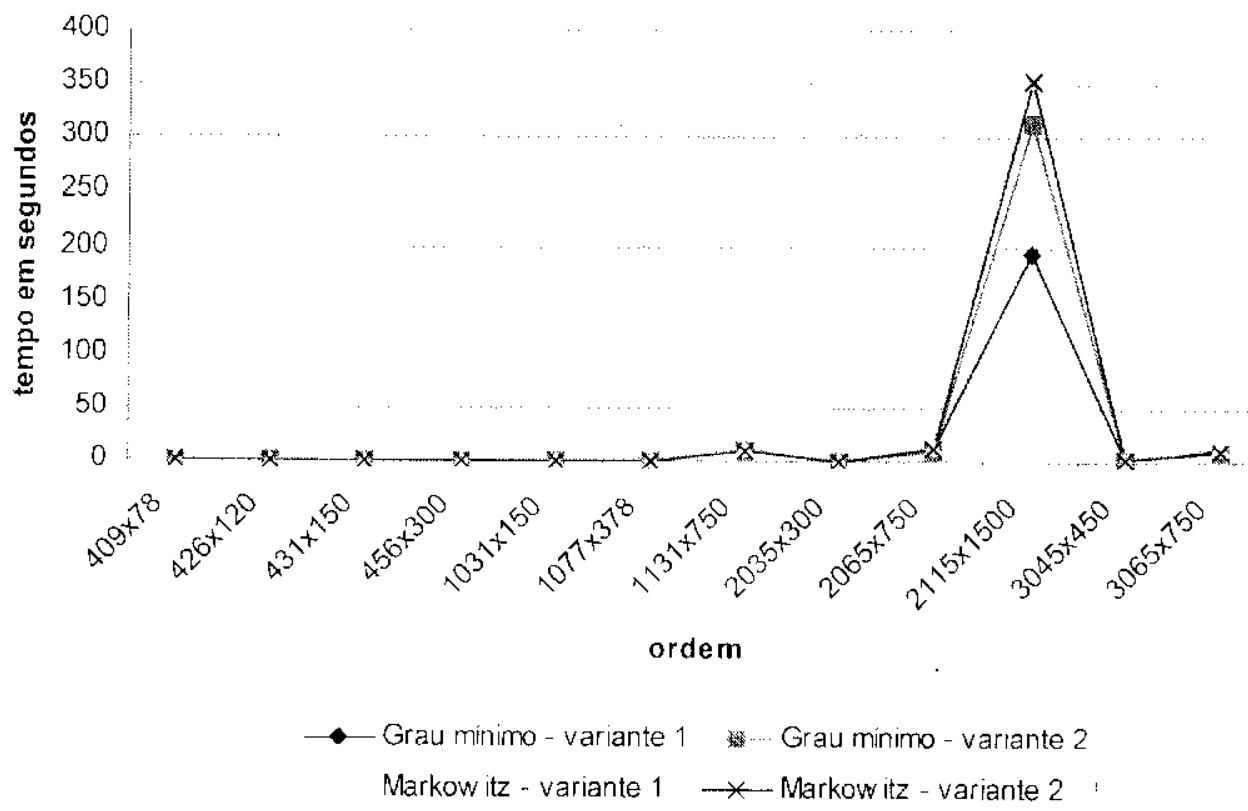

Figura 6.21 - Tempo por decomposição usando gerador bloco angular em vários níveis 


\begin{tabular}{|c|c|c|c|c|c|c|}
\hline \multirow[b]{3}{*}{ Problemas } & \multicolumn{6}{|c|}{ Tempo por atualização em segundos } \\
\hline & \multirow[b]{2}{*}{ Iterações } & \multirow[b]{2}{*}{$\% n z$} & \multicolumn{2}{|c|}{ Grau Mínimo } & \multicolumn{2}{|c|}{ Markowitz } \\
\hline & & & Variante 1 & Variante 2 & Variante 1 & Variante 2 \\
\hline $110 \times 400$ & 140,5 & 1.76 & 0,008 & 0 & 0.006 & 0 \\
\hline $160 \times 400$ & 129,2 & 1,66 & 0 & 0 & 0 & 0 \\
\hline $191 \times 400$ & 134,1 & 1,6 & 0.001 & 0 & 0 & 0 \\
\hline $241 \times 400$ & 119,8 & 1,87 & 0 & 0 & 0 & 0 \\
\hline $215 \times 1000$ & 258,7 & 1,8 & 0.078 & 0,091 & 0,034 & 0,067 \\
\hline $457 \times 1008$ & 310,1 & 1,62 & 0.095 & 0,092 & 0,041 & 0.055 \\
\hline $883 \times 1000$ & 509,6 & 1,36 & 0,053 & 0,05 & 0,037 & 0.022 \\
\hline $255 \times 2000$ & 424,8 & 1,88 & 0,882 & 0,718 & 0.792 & 0,712 \\
\hline $420 \times 2000$ & 663,8 & 0,76 & 0,74 & 0.526 & 0,736 & 0,616 \\
\hline $840 \times 2000$ & 687,2 & 0,72 & 0.724 & 0.722 & 0,69 & 0,564 \\
\hline $580 \times 3000$ & 792,4 & 0,76 & 1,908 & 1.52 & 1,782 & 1,344 \\
\hline $860 \times 3000$ & 751.6 & 1,12 & 1.858 & 1,192 & 1,776 & 1,3 \\
\hline $409 \times 78$ & 55,2 & $1,65 !$ & 0 & 0 & 0 & 0 \\
\hline $426 \times 120$ & 82,6 & 1,55 & 0 & 0 & 0 & 0 \\
\hline $431 \times 150$ & 105,8 & 1,49 & 0 & 0 & 0 & 0 \\
\hline $456 \times 300$ & 204,4 & 1,29 & 0 & 0 & 0 & 0 \\
\hline $1031 \times 150$ & 101,6 & 1,72 & 0 & 0 & 0 & 0 \\
\hline $1077 \times 378$ & 253,6 & 1,49 & 0 & 0 & 0 & 0 \\
\hline $1131 \times 750$ & 591,2 & 1,3 & 0,016 & 0.01 & 0.02 & 0,01 \\
\hline $2035 \times 300$ & 215 & 1,28 & 0 & 0 & 0 & 0 \\
\hline $2065 \times 750$ & 531,8 & 0,91 & 0.02 & 0.014 & 0,02 & 0,02 \\
\hline $2115 \times 1500$ & 1182,8 & 0,68 & 0.136 & 0,236 & 0,27 & 0,278 \\
\hline $3045 \times 450$ & 306,8 & 1,28 & 0,01 & 0,002 & 0,01 & 0 \\
\hline $3065 \times 750$ & 506.2 & 1,07 & 0.02 & 0,014 & 0,02 & 0,014 \\
\hline
\end{tabular}

Tabela 6.24 - Tempo por atualização usando gerador bloco angular em vários niveis

\begin{tabular}{|c|c|c|c|c|c|c|}
\hline \multicolumn{7}{|c|}{ Tempo por decomposição em segundos } \\
\hline \multirow[b]{2}{*}{ Problemas } & \multirow[b]{2}{*}{ Iterações } & \multirow[b]{2}{*}{$\% n z$} & \multicolumn{2}{|c|}{ Grau Minimo } & \multicolumn{2}{|c|}{ Markowitz } \\
\hline & & & Variante 1 & Variante 2 & Variante 1 & Variante 2 \\
\hline $110 \times 400$ & 140,5 & 1,76 & 0,019 & 0,014 & 0,02 & 0,018 \\
\hline $160 \times 400$ & 129,2 & 1.66 & 0,018 & 0,016 & 0,02 & 0.017 \\
\hline $191 \times 400$ & 134,1 & 1.6 & 0,015 & 0,014 & 0.019 & 0,015 \\
\hline $24 \uparrow \times 400$ & 119,8 & $1,87 j$ & 0.016 & 0,016 & 0,019 & 0,019 \\
\hline $215 \times 1000$ & 258,7 & 1,8 & 0.4 & 0,339 & 0,41 & 0,402 \\
\hline $457 \times 1008$ & 310,1 & 1,62 & 0,489 & 0,4 & 0.515 & 0,471 \\
\hline $883 \times 1000$ & 509.6 & 1,36 & 0.482 & 0,434 & 0.524 & 0,435 \\
\hline $255 \times 2000$ & 424,8 & 1,88 & 2,834 & 2,614 & 3.374 & 3,06 \\
\hline $420 \times 2000$ & 663,8 & 0.76 & 2,894 & 2,59 & 2.98 & 2.79 \\
\hline $840 \times 2000$ & 687,2 & 0,72 & 2,96 & 2,706 & 3,248 & 3,182 \\
\hline $580 \times 3000$ & 792,4 & 0.76 & 7,608 & 6.988 & 7.144 & 6.816 \\
\hline $860 \times 3000$ & 751,6 & 1.12 & 6.852 & 6,198 & 6.336 & 6,52 \\
\hline $409 \times 78$ & 55,2 & 1,65 & 0 & 0 & 0 & 0 \\
\hline $426 \times 120$ & 82,6 & 1,55 & 0 & 0 & 0 & 0 \\
\hline $431 \times 150$ & 105,8 & 1,49 & 0 & 0.002 & 0,002 & 0,004 \\
\hline $456 \times 300$ & 204.4 & 1,29 & 0.01 & 0,01 & 0.01 & 0,01 \\
\hline $1031 \times 150$ & 101.6 & 1,72 & 0,004 & 0 & 0,002 & 0 \\
\hline $1077 \times 378$ & 2536 & 1,49 & 0,02 & 0,012 & 0.012 & 0,016 \\
\hline $1131 \times 750$ & 591,2 & 1,3 & 0,112 & 0,084 & 0.132 & 0,11 \\
\hline $2035 \times 300$ & 215 & 1.28 & 0,012 & 0,01 & 0,012 & 0,012 \\
\hline $2065 \times 750$ & 531,8 & 0.91 & 0.106 & 0,086 & 0.13 & 0.114 \\
\hline $2115 \times 1500$ & 1182,8 & 0,68 & 1.534 & 1.402 & 1.634 & 1,498 \\
\hline $3045 \times 450$ & 306.8 & 1,28 & 0.024 & 0.02 & 0,026 & 0,024 \\
\hline $3065 \times 750$ & 506,2 & 1,07 & 0.104 & 0,084 & 0,136 & 0,108 \\
\hline
\end{tabular}

Tabela 6.25 - lempo por decomposição usando gerator bloco angular em vários niveis 
De acordo com as tabelas 6.23 e 6.24. para a maioria dos problemas no caso em que $m \cdot n$ e para os problemas pequenos no caso em que $n>m$, o tempo total de resolução e o tempo gasto por atualização. das combinações Grau Mínimo con a variante 2 c Markowit» com a variante 2. foram mais próximos e melhores. mas entre estas duas combinações. a primeira foi a melhor. Para os problemas maiores no caso em que $n>m$ houveram trocas entre as combinações com valores próximos, porém a combinação que apresentou um melhor desempenho foi Markowitz com a variante 2.

Com relação ao tempo gasto por decomposição, de acordo com a tabela 6.25 , não houveram diferenças suficientes para dizer que certas combinações tiveram resultados próximos e melhores, como anteriormente. Pode-se dizer apenas, que a combinaçăo Grau Mínimo com a variante 2 foi a melhor em todos os problemas no caso em que $n$ " $m$ e na maioria dos problemas no caso em que $m>n$.

Podemos observar pelas tabelas $6.23,6.24$ × 6.25 que o tempo total de resolução e o tempo gasto por atualização e por decomposição foram menores no caso em que $m>n$ do que no caso em que $n$ m em todos os problemas.

\begin{tabular}{|c|c|c|c|c|c|c|}
\hline \multicolumn{7}{|c|}{ Preenchimento por atualização } \\
\hline \multirow[b]{2}{*}{ Problemas } & \multirow[b]{2}{*}{ Iterações } & \multirow[b]{2}{*}{$\% \mathrm{nz}$} & \multicolumn{2}{|c|}{ Grau Mínimo } & \multicolumn{2}{|c|}{ Markowitz } \\
\hline & & & Variante 1 & Variante 2 & Variante 1 & Variante 2 \\
\hline $110 \times 400$ & 140,5 & 1,76 & 15,013 & 1471 & 7,294 & 6.504 \\
\hline $160 \times 400$ & 129,2 & 1,66 & 13,323 & 12,301 & 7,825 & $7: 103$ \\
\hline $191 \times 400$ & 134,1 & 1.6 & 11.578 & 11,496 & 7,322 & 6.74 \\
\hline $241 \times 400$ & 119,8 & 1,87 & 12.335 & 11,83 & 7,026 & 6,682 \\
\hline $215 \times 1000$ & 258,7 & 1,8 & 37.273 & 36,843 & 11,297 & 9,464 \\
\hline $457 \times 1008$ & 310,1 & 1,62 & 28.336 & 24,122 & 9,006 & 7,52 \\
\hline $883 \times 1000$ & 509.6 & 1,36 & 19,789 & 20,574 & 5.6356 & 4,507 \\
\hline $255 \times 2000$ & 424,8 & 1,88 & 72,992 & 70,92 & 15,304 & 12,882 \\
\hline $420 \times 2000$ & 663,8 & 0,76 & 52,77 & 52.882 & 10.244 & 8,698 \\
\hline $840 \times 2000$ & 687,2 & 0.72 & 44,434 & 48,592 & 10.644 & $8: 712$ \\
\hline $580 \times 3000$ & 792,4 & 0,76 & 81,252 & 79.74 & 12,842 & 10,264 \\
\hline $860 \times 3000$ & 751,6 & 1,12 & 69,924 & 69,618 & 11,128 & 9,244 \\
\hline $409 \times 78$ & 55,2 & 1,65 & 1.844 & 1.936 & 1,844 & 1,936 \\
\hline $426 \times 120$ & 82,6 & 1,55 & 1,812 & 1,94 & 1,812 & 1,94 \\
\hline $431 \times 150$ & 105,8 & 1,49 & 2.12 & 1,98 & 2,006 & 1,928 \\
\hline $456 \times 300$ & 204,4 & 1,29 & 4.608 & 4,358 & 2,106 & 1.63 \\
\hline $1031 \times 150$ & 101.6 & 1,72 & 1.562 & 2,006 & 1,518 & 1,962 \\
\hline $1077 \times 378$ & 253,6 & 1,49 & 4,22 & 3.992 & 2,054 & 1.704 \\
\hline $1131 \times 750$ & 591,2 & 1,3 & 6,344 & $6: 168$ & 1.64 & 1.47 \\
\hline $2035 \times 300$ & 215 & 1,28 & 7,688 & 7,268 & 3.01 & 2.02 \\
\hline $2065 \times 750$ & 531,8 & $0,91^{\prime}$ & 9.362 & 9.9 & 2,484 & 1.908 \\
\hline $2115 \times 1500$ & 1182.8 & 0,68 & 11.702 & 10,94 & 1,85 & 1,562 \\
\hline $3045 \times 450$ & 306,8 & 1,28 & 7.31 & 7,34 & 2,524 & 2,046 \\
\hline $3065 \times 750$ & 506,2 & 1,07 & 9.596 & 9.596 & 2,334 & 2,012 \\
\hline
\end{tabular}

Tabela 6.26 - Preenchimento por atualização usando geverador bloco angular em vários niveis 


\section{Preenchimento por atualização $(n>m)$}

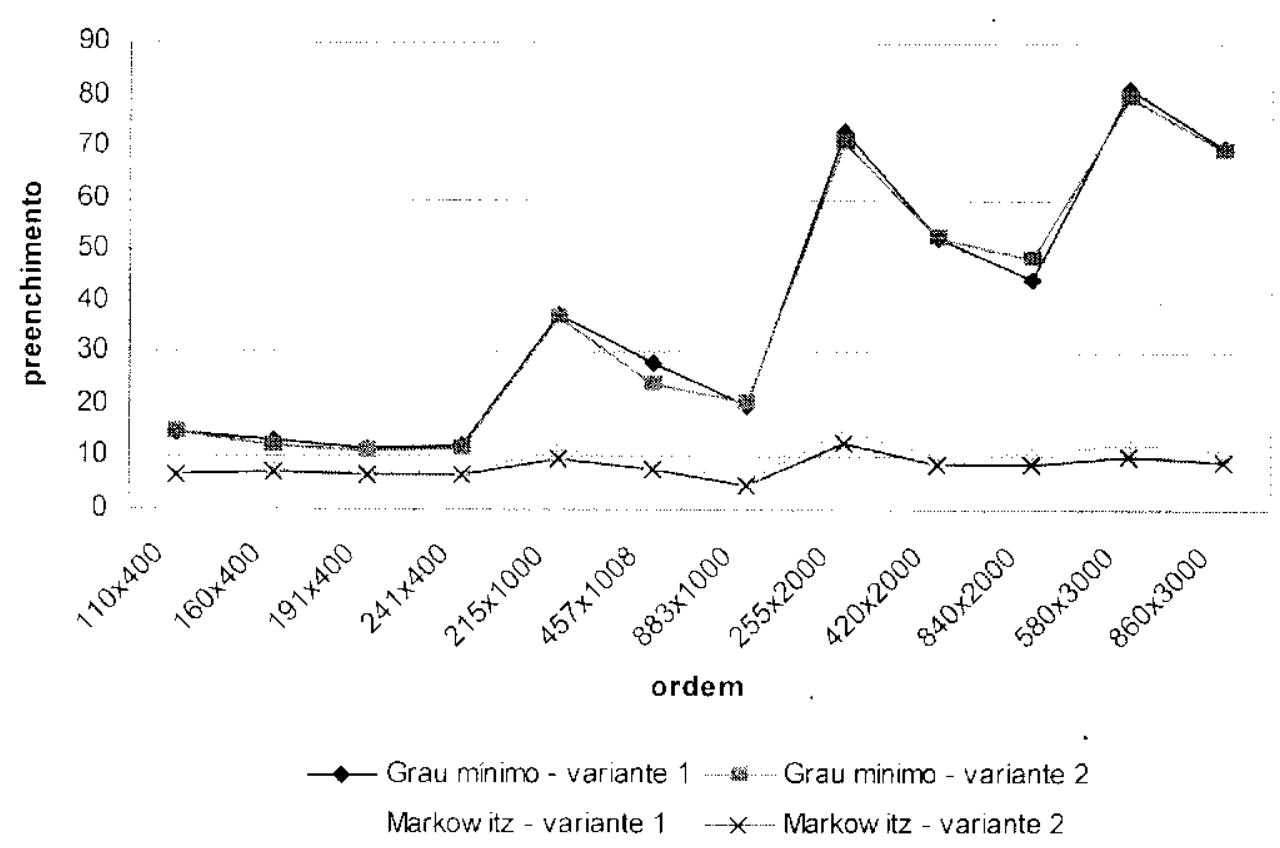

Figura 6.22 - Preenchimento por atualizaçào usando gerador bloco angular em vários níveis

\section{Preenchimento por atualização $(m>n)$}

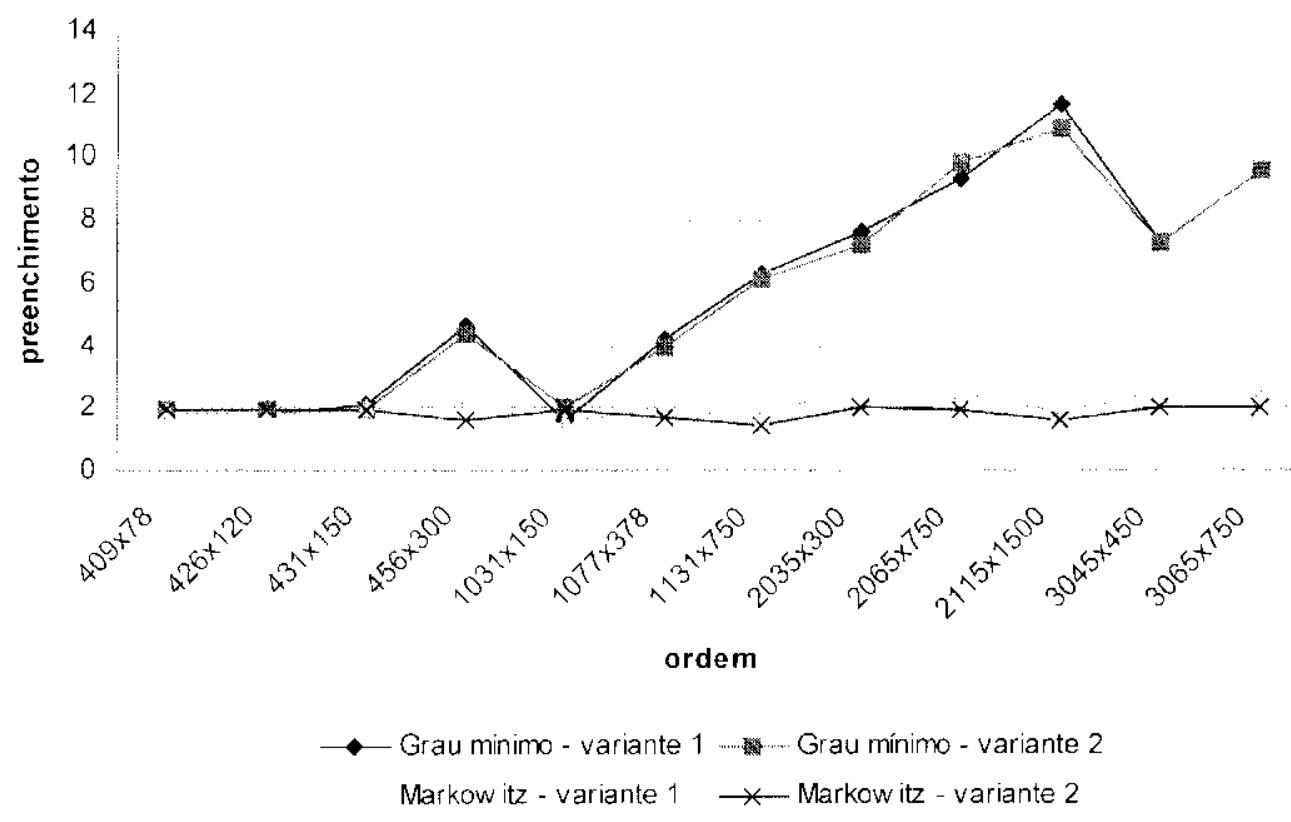

Figura 6.23 - Preenchimento por atualização usando gerador bloco angular em vários niveis 


\begin{tabular}{|c|c|c|c|c|c|c|}
\hline \multirow{3}{*}{ Problemas } & \multirow{2}{*}{ - } & \multicolumn{3}{|c|}{ Preenchimento por decomposiçäo } & \multirow{2}{*}{\multicolumn{2}{|c|}{ Markowitz }} \\
\hline & & & \multicolumn{2}{|c|}{ Grau Mínimo } & & \\
\hline & Iteraçōes & $\% n z$ & Variante 1 & Variante 2 & Variante 1 & Variante 2 \\
\hline $110 \times 400$ & $140: 5$ & 1,76 & 10.25 & 0.45 & 0,1 & 0.3 \\
\hline $160 \times 400$ & 129.2 & 1,66 & 13,75 & 4.85 & 1,35 & 1,85 \\
\hline $191 \times 400$ & 134.1 & 1,6 & 24,55 & 20,35 & 2,55 & 2.45 \\
\hline $241 \times 400$ & 119.8 & 1,87 & 7,25 & 5,15 & 0,35 & 0,1 \\
\hline $215 \times 1000$ & 258,7 & 1,8 & 23,13 & 35.93 & 1,234 & 1.566 \\
\hline $457 \times 1008$ & 310,1 & 1,62 & 99,02 & 99,635 & 2.375 & 1,933 \\
\hline $883 \times 1000$ & 509,6 & 1,36 & 58,957 & 61,03 & $2: 289$ & 2,58 \\
\hline $255 \times 2000$ & 424,8 & 1.88 & 200,08 & 235,8 & 6,84 & 4,8 \\
\hline $420 \times 2000$ & 663,8 & 0.76 & 170.468 & 140,558 & 4.03 & 2,574 \\
\hline $840 \times 2000$ & 687,2 & 0.72 & 108,096 & 117,618 & 3.314 & 3,044 \\
\hline $580 \times 3000$ & 792,4 & 0.76 & 286.826 & 195,74 & 2,856 & 2,924 \\
\hline $860 \times 3000$ & 751,6 & 1,12 & 0 & 0 & 0 & 0,352 \\
\hline $409 \times 78$ & 55,2 & 1,65 & 0 & 0 & 0 & 0 \\
\hline $426 \times 120$ & 82,6 & 1,55 & 0 & 0 & 0 & 0 \\
\hline $431 \times 150$ & 105,8 & 1,49 & 0 & 0 & 0 & 0 \\
\hline $456 \times 300$ & 204.4 & 1,29 & 0 & 0 & 0 & 0 \\
\hline $1031 \times 150$ & 1016 & 1,72 & 0 & 0 & 0 & 0 \\
\hline $1077 \times 378$ & 253.6 & 1,49 & 0 & 0 & 0 & 0 \\
\hline $1131 \times 750$ & 591.2 & 1,3 & 0 & 4 & 0 & 0 \\
\hline $2035 \times 300$ & 215 & 1,28 & 7.334 & 1 & 0,134 & 0,134 \\
\hline $2065 \times 750$ & 531.8 & 0,91 & 0 & 2,1 & 0,566 & 0 \\
\hline $2115 \times 1500$ & 1182,8 & 0,68 & 2.076 & 11.772 & 0.164 & 0,358 \\
\hline $3045 \times 450$ & 306.8 & 1,28 & 4 & 0 & 0 & 0.25 \\
\hline $3065 \times 750$ & 506.2 & 1,07 & 2.08 & 12.166 & 0 & 0,414 \\
\hline
\end{tabular}

Tabela 6.27 - Preenchimento por decomposição usando gerador bloco angular em vários niveis

Analisando a tabela 6.26. vemos que. em relação ao precnchimento por atualização, as combinações Markowitz com a variante 1 e Markowitz com a variante 2 apresentaram resultados próximos e melhores para a maioria dos problemas no caso $\mathrm{cm}$ que $n \geqslant m$ e para os problemas maiores no caso em que $m>n$. Houve uma troca dos melhores resultados entre as combinações para os problemas menores no caso en que $m>n$. F de acordo com a tabela 6.27, para o preenchimento por decomposição as melhores combinaçóes foram Markowitz com a variante 1 e Markowitz com a variante 2, sendo que a primeira foi mais rápida na maioria dos problemas. Os preenchimentos feitos por atualização e deconposição usando a heuristica de Grau Mínimo foram bem superiores no caso em que $n \times m$. Para a heuristica de Markowitz os preenchimentos feitos por atualizaçâo e decomposição foram maiores no caso $\mathrm{cm}$ que $n \times m$. Os preenchimentos reitos tanto na atualização quanto na decomposição usando a heurística de Grau Minimo foram maiores em quase todos os problemas.

Lim geral:

- Melhor Combinação: Markowilz com a variante 2.

- Pior Combinação: Grau Mínimo com a variante 1. 
De acordo com as tabelas 6.7 a 6.27 resumimos na tabela 6.28 quais foram as melhores c piores combinações para todos os itens analisados, nos casos em que $n>m$ e $m>n$. com relação aos quatro geradores utilizados.

\begin{tabular}{|c|c|c|c|c|c|c|c|c|}
\hline & & Melhores & s combinaçc & öes para $n$ & $n>m$ & & & \\
\hline & Produc & lçăo & Bloco An & ngular & Esca & ada & Bloco Ang. vá & ários niveis \\
\hline & Heuristica & Variante & Heuristica & Variante & Heuristica & Variante & Heurística & Variante \\
\hline Tempo de resolução & M & 2 & M & 2 & G & 2 & $M$ & 2 \\
\hline Preenchimento/atualização & $M$ & 2 & M & 2 & $M$ & 1 & $M$ & 2 \\
\hline Tempo/atualizaçäo & M & 2 & $M$ & 2 & $\mathrm{G}$ & 2 & M & 2 \\
\hline Preenchimento/decomposição & $\mathrm{M}$ & 1 & $M$ & 2 & M & 1 e 2 & M & $1 \mathrm{e} 2$ \\
\hline Tempo/decomposição & $\mathrm{G}$ & 2. & G & 2 & G & 2 & G & 2 \\
\hline & & elhores co & ombinações & para $m>$ & $n$ & & & \\
\hline & Produs & ção & Bloco An & ngular & Esca & da & Bloco Ang. vá & ários niveis \\
\hline & Heurística & Variante & Heuristica & Variante & Heuristica & Variante & Heurística & Variante \\
\hline Tempo de resolução & -- & -- & M. & 2 & $\mathrm{G}$ & 2 & M & 2 \\
\hline Preenchimento/atualização & -- &.- & $\mathrm{M}$ & 2 & M & 1. & M & 2 \\
\hline Tempo/atualização & -- & -- & $G$ & 2 & G & 2 & $M e G$ & 2. \\
\hline Preenchimento/decomposição & -- & -- & $\mathrm{M}$ & 1 & $\mathrm{G}$ & $1 \mathrm{e} 2$ & M & 1 \\
\hline Tempo/decomposição & -- & - & G & 2 & $\mathrm{G}$ & 2 & $\mathrm{G}$ & 2 \\
\hline & & Piores $\mathrm{c}$ & combinaçöes & s $n>m$ & & & & \\
\hline & Produs & ção & Bloco An & ngular & Escas & da & Bloco Ang. vá & ários níveis \\
\hline & Heuristica & Variante & Heuristica & Variante & Heuristica & Variante & Heurística & Variante \\
\hline Tempo de resolução & M & 1 & G. & 1 & M & 1 & G & 1 \\
\hline Preenchimento/atualizaçäo & $\mathrm{G}$ & 1 & $\mathrm{G}$ & 1 & $\mathrm{G}$ & 2 & $\mathrm{G}$ & 1 \\
\hline Tempo/atualização & M & 1 & $\mathrm{G}$ & 1 & M & 1 & G & 1 \\
\hline Preenchimento/decomposição & G & 1 & G & 1 & $\mathrm{G}$ & 1 e 2 & G & 1 e 2 \\
\hline Tempo/decomposição & $\mathrm{M}$ & 1 & $G$ & 1 & M & 1 & M & 1 \\
\hline & & Piores c & combinaçōe & es $m>n$ & & & & \\
\hline & Produc & ção & Bloco An & ngular & Esca & da & Bloco Ang. vá & ários niveis \\
\hline & Heurística & Variante & Heuristica & Variante & Heuristica & Variante & Heuristica & Variante \\
\hline Tempo de resoluçāo & $\therefore$ & -- & M & 1 & M & 1 & M & 1 \\
\hline Preenchimento/atualização & -- & -- & G & 1 & $\mathrm{G}$ & 2 & G & 2 \\
\hline Tempo/atualização & -- &.$- \cdot$ & $\mathrm{MeG}$ & 1 & M & 1 & M & 1. \\
\hline Preenchimento/decomposição & -- & -- & $\mathrm{G}$ & 1 & M & $1 € 2$ & G & $\underline{2}$ \\
\hline Tempo/decomposição & -- & -- & M & 1 & M & 1 & $\mathrm{M}$ & 1 \\
\hline
\end{tabular}

Tabela 6.28 - Comparação dos resultados entre us quatro geradores

()bservando a tabela 6.28 , podemos concluir que a combinação heurística de Markowity. com a variante 2 apresentou melhores resultados na maioria dos itens analisados. considerando os dois casos $(n+m \mathrm{e} m n$ ) e em relação aos quatro geradores utilizados. Podemos observar ainda que a heuristica de Cirau Mínimo com a variante 2 foi a melhor combinação para o tempo de decomposição e a heurística de Markowitz con a variante 1 foi a 
pior. Em relação ao tempo de resolução e de atualização a melhor combinação foi a heurística de Markowit\% com a variante 2 e a pior combinação foi a heurística de Markowit\% com a variante 1 . Os precnchimentos por atualização e decomposição, no geral, foram menores com a combinação heurística de Markowitz com a variante 2 e maiores com a combinação heuristica Grau Mínimo com a variante 1. Una última observação é que os resultados das combinações heurística de Markowity com as variantes 1 e 2 são melhores e bem mais estáveis que os resultados obtidos com as combinações Grau Mínimo com variantes 1 e $2 \mathrm{em}$ relação ao preenchimento por atualização, como pode ser visto pelos gráticos.

\section{Comparação entre o método dual canalizado com busca linear por partes e o software CPLEX 7.1}

Comparamos agora o método dual simplex canalizado com busca linear por partes usando os quatro geradores com o software CPLEX 7.1 para MS Windows com relação ao número de iteraçôes e tempo de resolução, utilizando os mesmos problemas e as melhores combinaçôs entre heurísticas e variantes para cada gerador, identiticadas anteriormente. $O$ (PI.IXX foi utilizado sem ativar o pré-processador. foi usado o método dual simplex (iranopt) para resolver os problemas e o valor para o número de atualizações foi 100 , o mesmo usado pelo dual canalizado.

\begin{tabular}{|c|c|c|c|c|c|}
\hline \multicolumn{5}{|c|}{ Produção } \\
\hline \multicolumn{7}{|c|}{ Número de Iterações } & Tempo de Resolução em segundos \\
\hline Problemas & $\%$ nz & CPLEX 7.1 & Dual & CPLEX 7.1 & Dual \\
\hline $60 \times 100$ & 3,25 & 72.8 & 70,8 & 0 & 0,021 \\
$110 \times 200$ & 1,77 & 144.7 & 136,7 & 0.01 & 0.143 \\
$160 \times 300$ & 1,22 & 204.7 & 195,1 & 0.01 & 0.524 \\
$220 \times 400$ & 0,9 & 273,7 & 269,4 & 0,02 & 0.905 \\
$255 \times 500$ & 0.75 & 341,5 & 321,4 & 0,03 & 2,56 \\
$320 \times 600$ & 0,62 & 400,3 & 393,1 & 0,03 & 2,785 \\
$420 \times 800$ & 0,47 & 546 & 530,8 & 0,05 & 7,194 \\
$505 \times 1000$ & 0,38 & 672,4 & 643,2 & 0,08 & 38,608 \\
$620 \times 1200$ & 0,32 & 804,4 & 775 & 0,09 & 37,308 \\
$755 \times 1500$ & 0,25 & 1018 & 964,6 & 0,17 & 241,8 \\
$1010 \times 2000$ & 0,19 & 1415 & 1285.8 & 0,31 & 471,2 \\
$1210 \times 2400$ & 0,16 & 1708,4 & 1521,4 & 0.44. & 887,98 \\
\hline
\end{tabular}

Tabela 6.29 - Comparaçăo entre o CPLEX 7.1 e o Dual usando o gerador produção

Analisando a tabela 6.29 e a figura 6.24, podemos observar que o método dual simplex canalizado foi superior ao CPLEX em todos os problemas com relação ao número de iterações e também que, conforme foi aumentando a ordem dos problemas a diferença entre cles ficou maior. Lm se tratando do tempo de resolução, o CPLEX foi muito superior ao método dual canalizado. principalmente nos problemas maiores. 


\section{Comparação do número de iterações}

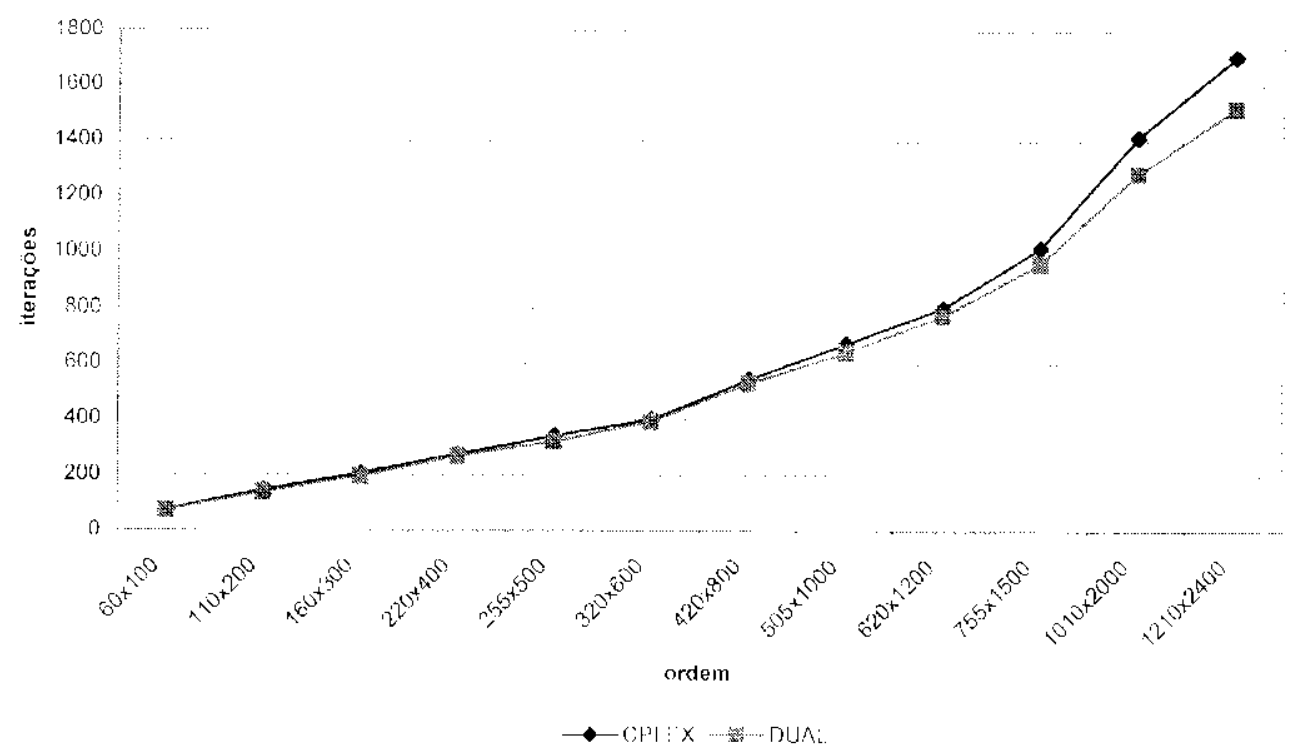

Figura 6.24 - Comparação entre o (Pl.t.X 7.1 e o Dual usando o gerador produção

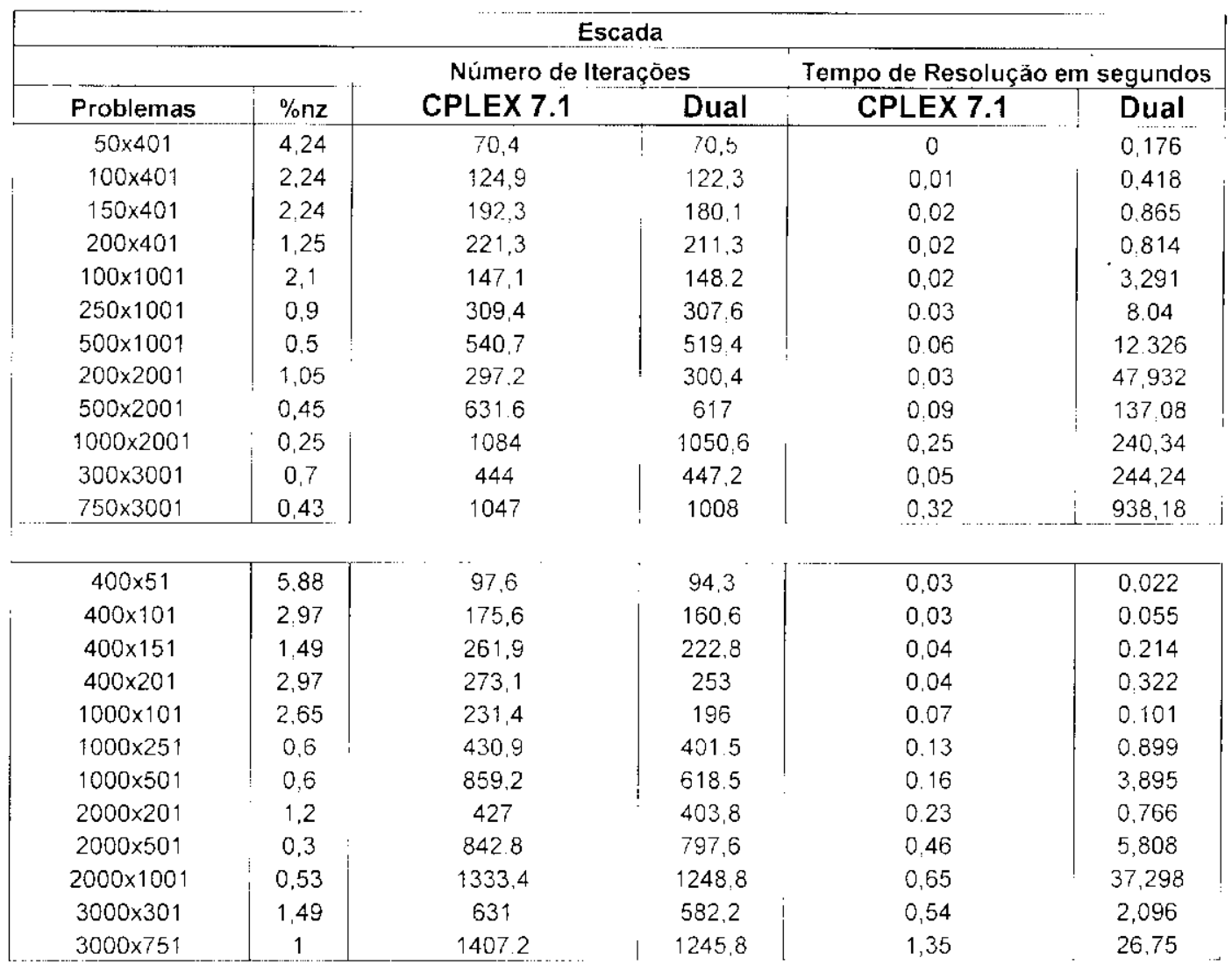

Tabela 6.30 - Comparação entre o CPI I: X 7.1 e o Dual usando o gerador escada 
Analisando a tabela $6.30 \mathrm{e}$ as figuras 6.25 e 6.26 . vemos que na maioria dos problemas o dual canalizado realizou um número menor de iterações. $A$ diferença entre eles foi maior nos problemas maiores onde $m>n$. Podemos observar também que $\mathrm{cm}$ alguns problemas o CPLJXX realizou menos iterações que o dual canalizado. Isto aconteceu nos problemas maiores no caso em que $n>m$ quando $m$ é aproximadamente $10 \%$ de $n$ e também em alguns problemas maiores no caso $\mathrm{em}$ que $m>n$ quando $n$ é aproximadamente $1 / 4 \mathrm{de} m$. O tempo de resolução do CPLEX novamente foi muito inferior ao tempo do dual canalizado. principalmente no caso $\mathrm{em}$ que $n=m$. Os problemas quando $m=n$ realizaram um número maior de iterações que os problemas quando $n>m$, porém o tempo total de resolução foi menor.

\section{Comparação do número de iterações $(n>m)$}

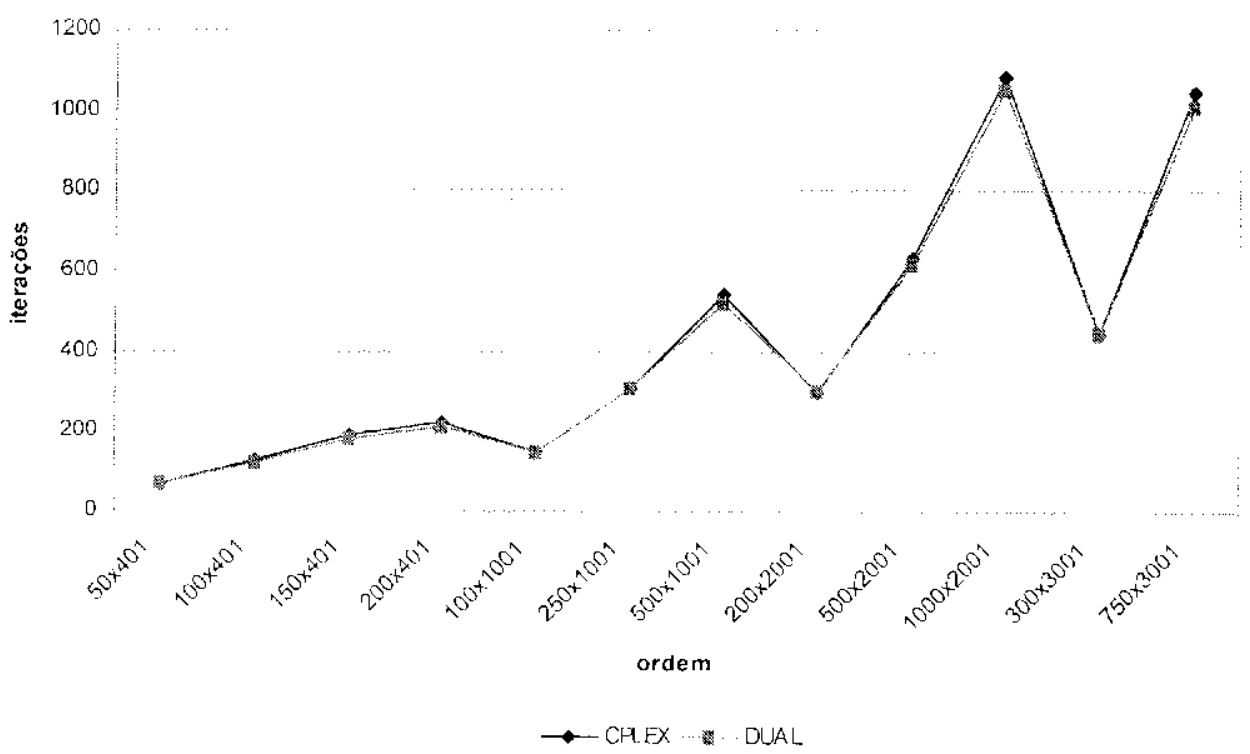

Figura 6.25 - Comparação entre o CPlılX 7.1 c o Dual usando o geraldor esceada 


\section{Comparação do número de iteraçōes $(m>n)$}

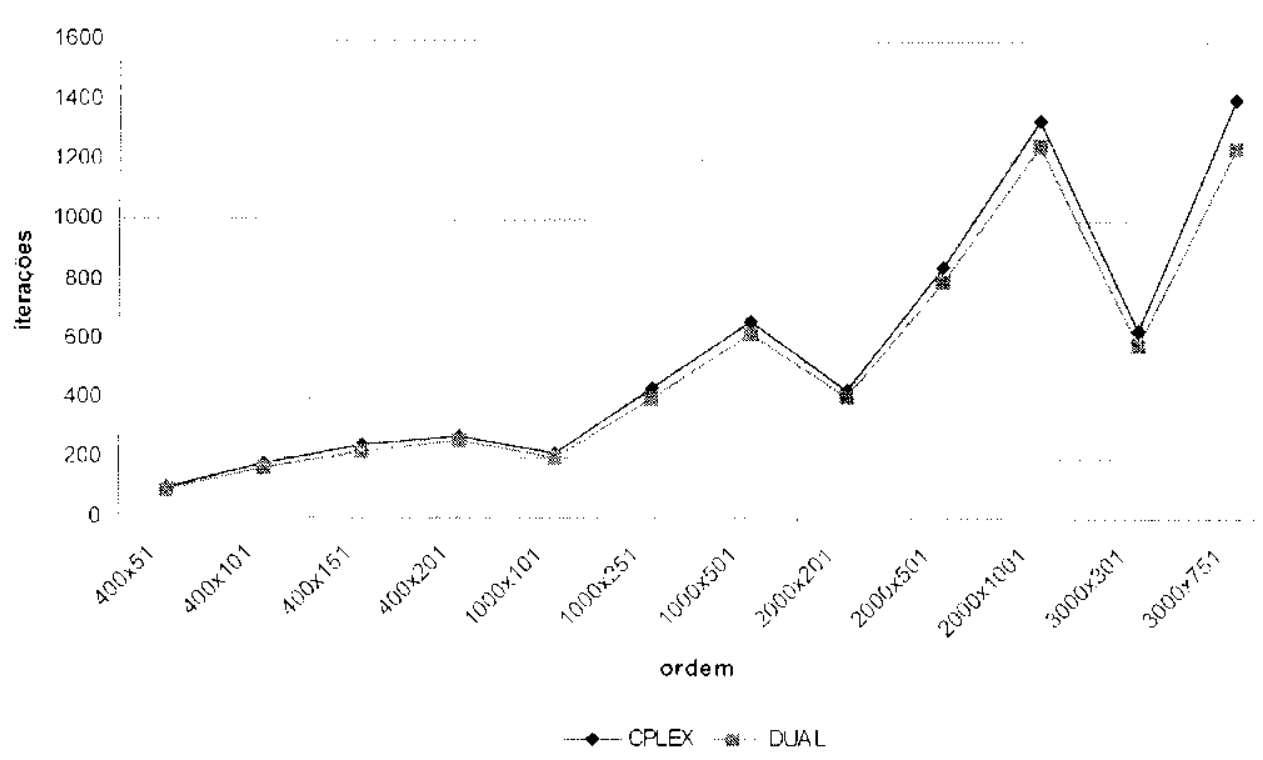

Figura 6.26 - Comparação entre o (PI.F.X 7.1 e o Dual usando o gerador escada

\begin{tabular}{|c|c|c|c|c|c|}
\hline \multicolumn{6}{|c|}{ Bloco Angular } \\
\hline \multirow[b]{2}{*}{ Problemas } & \multirow[b]{2}{*}{$\% \mathrm{nz}$} & \multicolumn{2}{|c|}{ Nümero de Iteraçöes } & \multicolumn{2}{|c|}{ Tempo de Resoluçāo em segundos } \\
\hline & & CPLEX 7.1 & Dual & CPLEX 7.1 & Dual \\
\hline $60 \times 400$ & 5 & 101,7 & $78: 7$ & 0.02 & 0.389 \\
\hline $90 \times 400$ & 3,33 & 124,1 & 116,3 & 0.02 & 0,802 \\
\hline $158 \times 400$ & 2,53 & 153,8 & 159,6 & 0,02 & 1,032 \\
\hline $204 \times 400$ & 1,47 & 95,6 & 87 & 0,02 & 0,247 \\
\hline $120 \times 1000$ & 2,5 & 296.5 & 324,625 & 0,04 & 49,826 \\
\hline $258 \times 1000$ & 1,16 & 201 & 175,8 & 0,03 & 11,031 \\
\hline $504 \times 1000$ & 0.6 & 227,8 & 210.7 & 0,04 & 7,604 \\
\hline $220 \times 2000$ & 1.36 & 363.2 & 377.6 & 0,06 & 317,3 \\
\hline $508 \times 2000$ & 0.59 & 362.4 & 308.6 & 0,08 & 115.57 \\
\hline $1004 \times 2000$ & 0.3 & 458,8 & $395: 8$ & 0.13 & 93,806 \\
\hline $320 \times 3000$ & 0,94 & 440,8 & 448.75 & 0.11 & 866.42 \\
\hline $762 \times 3000$ & 0,52 & 806,6 & $740: 75$ & 0.27 & 1105,15 \\
\hline $402 \times 50$ & 4,23 & 84,5 & 77,3 & 0,02 & 0,012 \\
\hline $402 \times 100$ & 2,24 & 144,4 & 134,7 & 0,03 & 0,056 \\
\hline $403 \times 150$ & 2,23 & 232,4 & 176.8 & 0,05 & 0,204 \\
\hline $402 \times 200$ & 1,24 & 184 & 197.7 & 0,03 & 0.225 \\
\hline $1002 \times 100$ & 2,1 & 218,1 & 172,2 & 0,07 & 0,093 \\
\hline $1002 \times 250$ & 0,9 & 390,9 & 336 & 0,12 & 0,726 \\
\hline $1002 \times 500$ & 0,5 & 467,3 & 418,2 & 0.11 & 2.271 \\
\hline $2002 \times 200$ & 1,05 & 529,2 & 357.4 & 0,27 & 0,706 \\
\hline $2002 \times 500$ & 0.45 & 757 & 662 & 0,36 & 4,248 \\
\hline $2002 \times 1000$ & 0.25 & 944.4 & 856.4 & 0,44 & 26.15667 \\
\hline $3002 \times 300$ & 0.7 & 826.4 & 573.6 & 0,66 & 2,25 \\
\hline $3003 \times 750$ & 0.43 & 1764 & 1269 & 1,67 & 39,74 \\
\hline
\end{tabular}

Tabela 6.31 - Comparação entre o (Pl.1.X 7.1 e o Dual usando o gerador bloco angular

De acordo com a tabela $6.31 \mathrm{c}$ as figuras $6.27 \mathrm{e} 6.28$. em quase todos os problemas o dual canalizado realizou menos iterações que o C PL.EX. exceto nos problemas maiores no caso em que $n \% m$ quando $m$ é aproximadamente $10 \%$ de $n$ e também em alguns problemas 
pequenos nos casos em que $n \approx m$ e $m \propto n$ quando a diferença entre $m$ e $n$ é aproximadamente $50 \%$. A dilerença entre o dual e o (Pl.l:X foi mais signilicativa nos problemas maiores quando $m>n$. Mais uma ve $z$ o tempo de resolução do CPLEX foi inferior ao tempo do dual canalizado, principalmente nos problemas maiores cos problemas quando $m>n$ apesar de' realizarem mais iterações foram mais rápido.

\section{Comparação do número de iterações $(n>m)$}

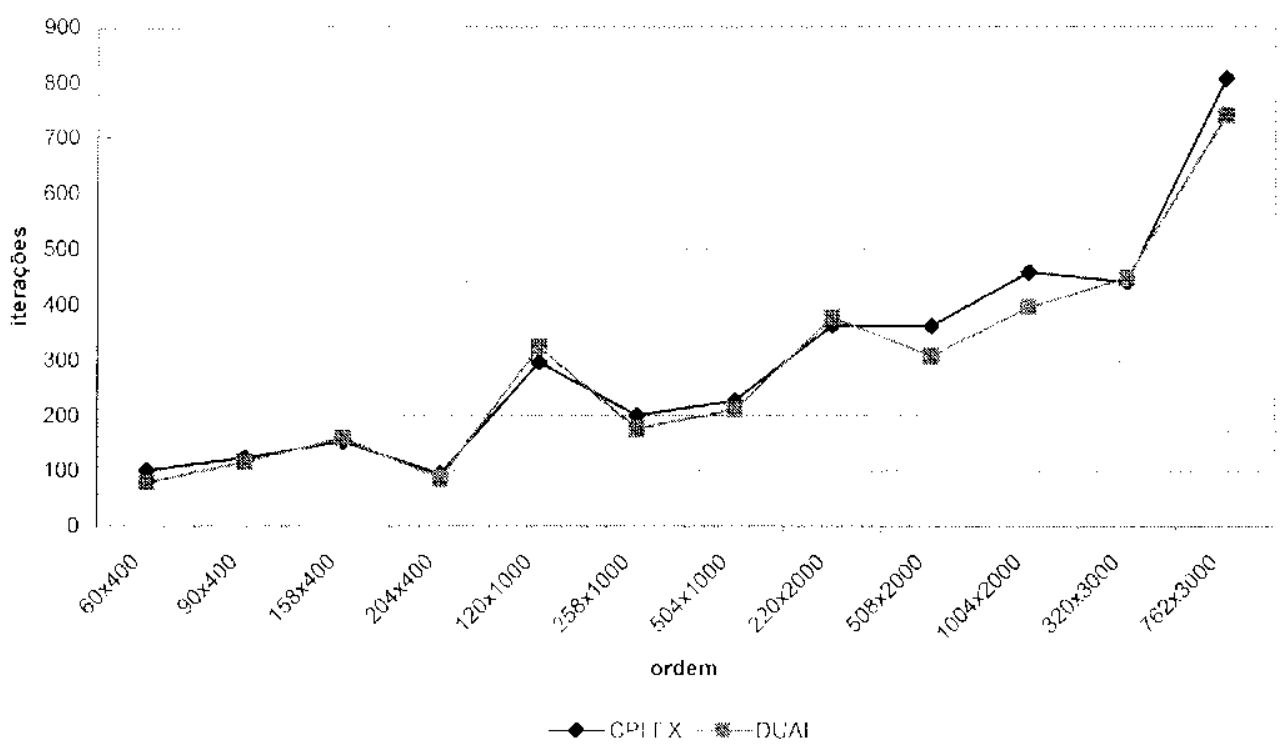

Figura 6.27 - Comparaçăo entre o CPLEX 7.1 e o Dual usando o gerador bloco angular

\section{Comparação do número de iterações $(m>n)$}

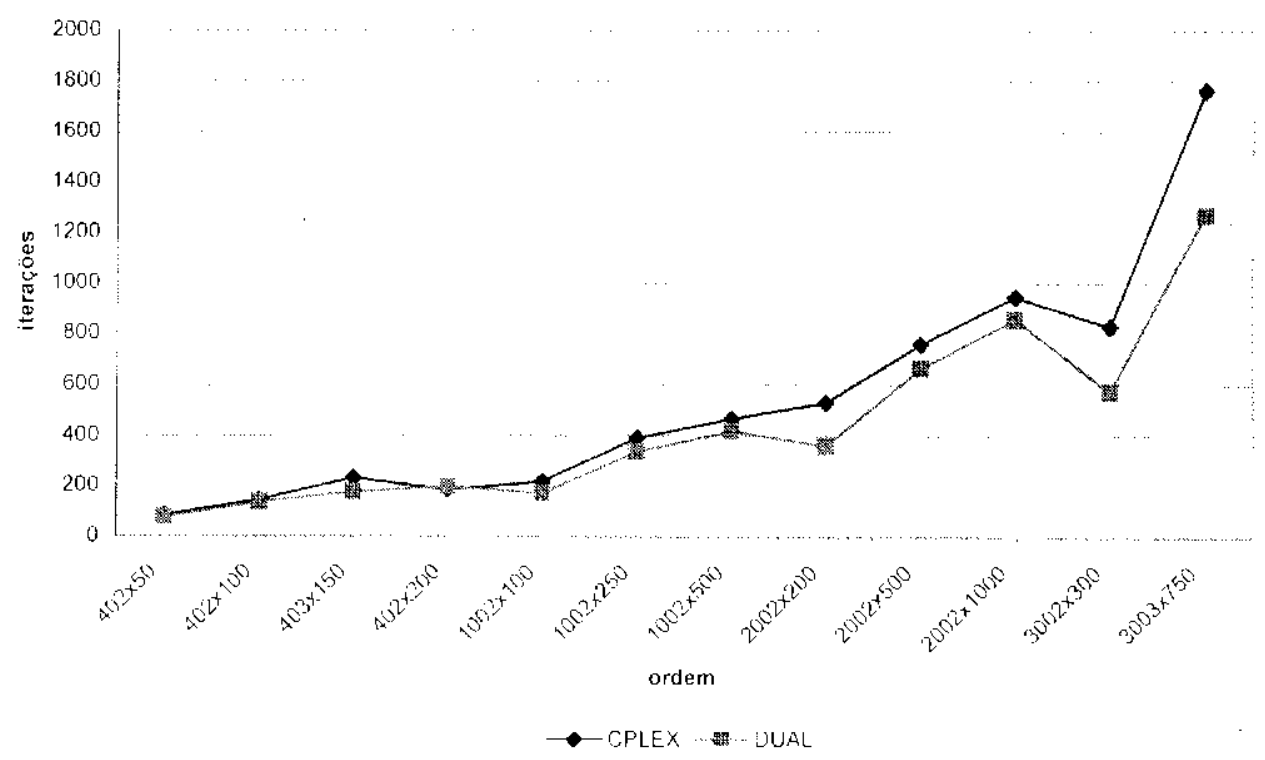

Figura 6.28 - Comparação entre o CPL.FX 7.1 e o Dual usando o gerador bloco angular 


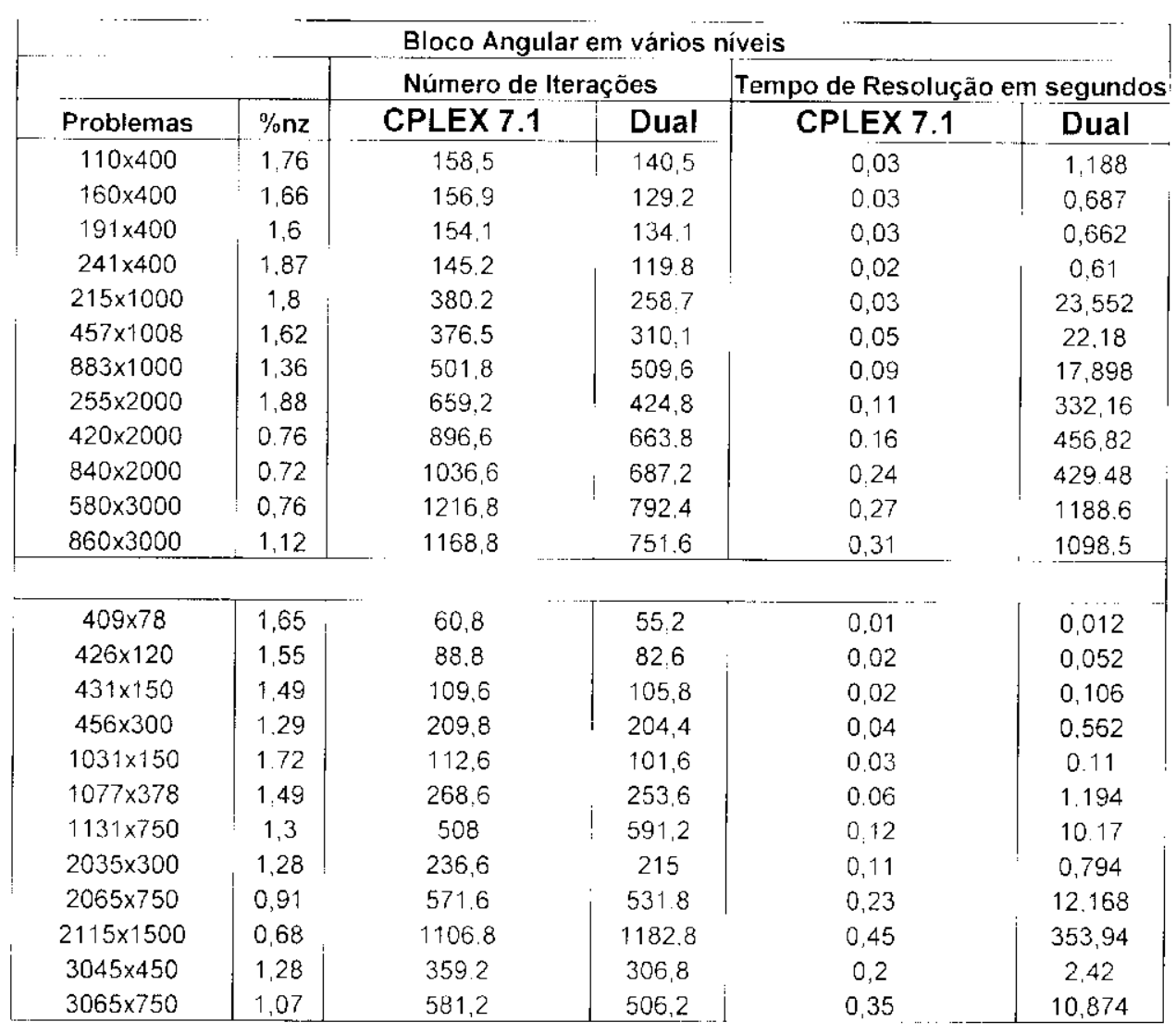

Tabela 6.32 - Comparaçĩo entre o CPLLX 7.1 co Dual usando o getador bloco angular em vários niveis

Pela tabela 6.32 e figuras 6.29 e 6.30 . podemos ver que o método dual simplex canalizado foi superior ao CPLEX en quase todos os problemas com relação ao número de iterações. exceto para alguns problemas em que a diferença entre $m$ e $n$ é maior que $65 \%$ para os casos em que $n>m$ e $m>n$ e também que a diferença entre o CPLEX co dual canalizado foi maior nos problemas onde $n>m$. Em se tratando do tempo de resolução. os resultados do CPll:X foram melhores que os resultados do método dual canalizado, principalmente, no cáso em que $n$ "m. Quando $m$. n lanto o número de iterações quanto o tempo de resolução loram meneres para a maioria dos problemas para o dual canalizado. 


\section{Comparação do número de iterações $(n>m)$}

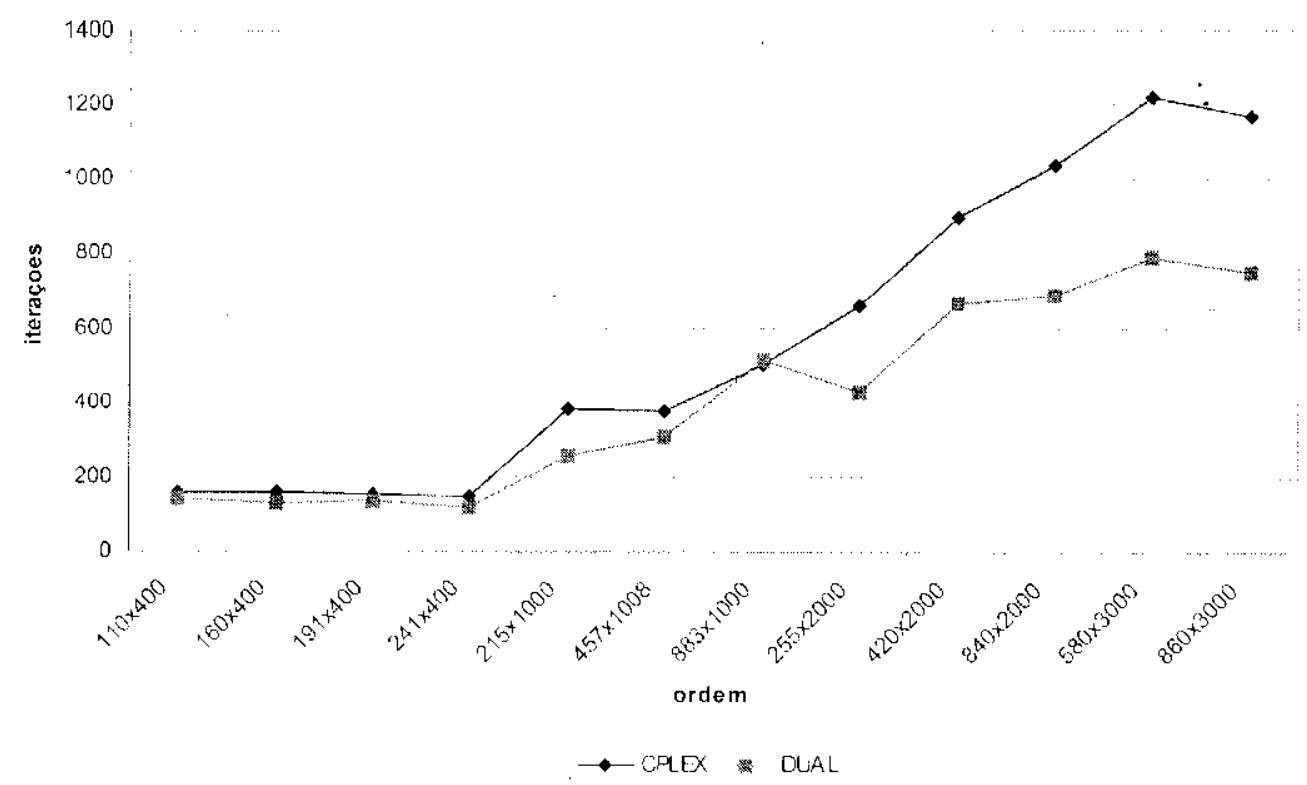

Figura 6.29 - Comparação entre o CPLLXX 7.1 e o Dual usando o gerador bloco angular em vários níveis

\section{Comparação do número de iterações $(m>n)$}

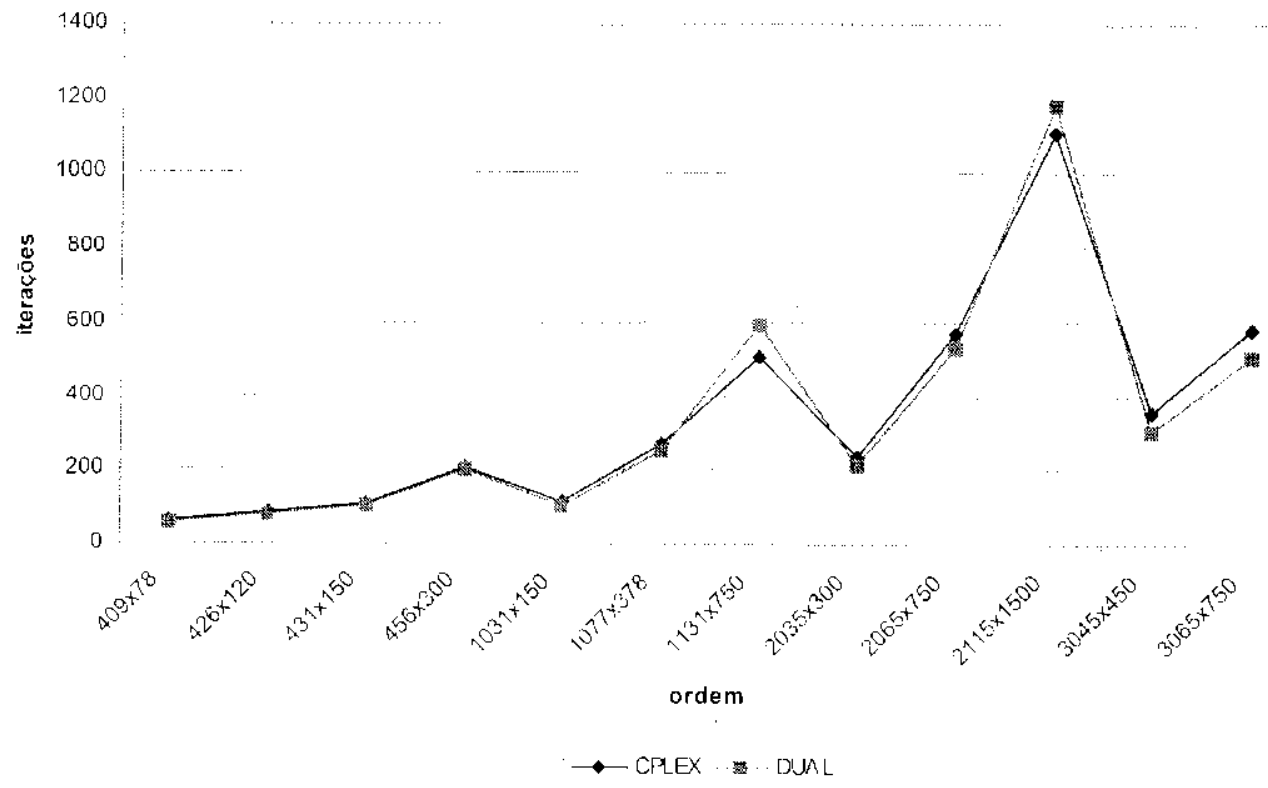

Figura 6.30 - Comparação entre o CPLEX 7.1 e o Dual usando o gerador bloco angular em vários níveis 


\section{Comparação entre a implementação do método dual simplex canalizado com busca linear por partes esparsa e a implementação densa.}

Neste momento comparamos o método dual simplex canalizado com busca linear por partes que explora esparsidade com o método que não explora. implementado por Sousa |37|. em relaçẫo ao tempo de resolução. utilizando as melhores combinaçós entré heurísticas e variantes para cada gerador e problemas cujos valores para $m$ e $n$ são inferiores a 400 . uma ve\% que a implementação feita por Soura [37] utiliza uma estrutura estática para armazenamento e ficou limitado quanto ao lamanho das matrizes (ordem inferior a $50(0)$ ).

\begin{tabular}{|c|c|c|c|c|}
\hline \multicolumn{5}{|c|}{ Produção } \\
\hline & & & Tempo de & soluçäo \\
\hline Problemas & Iteraçöes & $\% \mathrm{nz}$ & Esparso & Denso \\
\hline $30 \times 50$ & 40,4 & 6.3 & 0.01 & 0,03 \\
\hline $55 \times 100$ & 68,3 & 3.45 & 0.03 & 0.31 \\
\hline $80 \times 150$ & 100,6 & 2,38 & 0.08 & 1,36 \\
\hline $110 \times 200$ & 138,7 & 1,77 & 0,13 & 4,19 \\
\hline $160 \times 300$ & 192.4 & 1.22 & 0,42 & 21,38 \\
\hline $220 \times 400$ & 271.9 & 0.9 & 0.92 & 75,47 \\
\hline
\end{tabular}

Tabela 6.33 - Comparaçăo entre o dual canalizado esparso e denso usando o gerador produçà)

\section{Comparação do tempo de resolução}

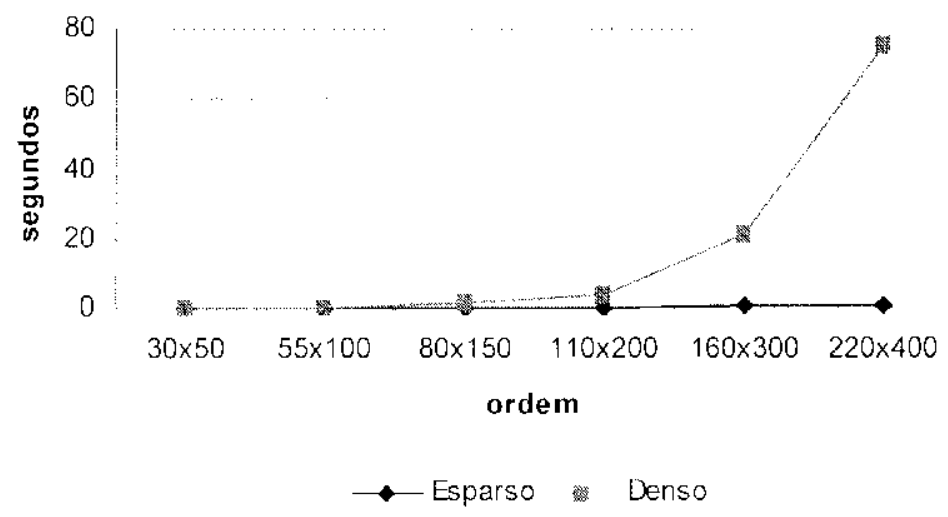

Figura 6.31 - Comparaçăo entre o dual canalizado esparso e denso usando o gerador produçào

De acordo com a tabela 6.33 e figura 6.31 podemos ver que o método dual canalizado que explora a esparsidade apresentou um tempo de resolução bastante inferior ao método que năo explora a esparsidade para lodos os problemas da produção. Observe mais claramente pelo gráfico que, quanto maior a ordem do problema. maior vai sendo a diferença entre os tempos de resoluçào. 


\begin{tabular}{|c|c|c|c|c|}
\hline \multirow[b]{3}{*}{ Problemas } & \multicolumn{3}{|c|}{ Bloco Angular } & \\
\hline & & & Tempo de resolução & \\
\hline & Iteraçōes & $\%$ nz & Esparso & Denso \\
\hline $54 \times 100$ & 31,4 & 5.56 & 0,01 & 0.13 \\
\hline $58 \times 200$ & 60,1 & 5.17 & 0.06 & 1.86 \\
\hline $104 \times 200$ & 64,5 & 2,88 & 0.03 & 2,04 \\
\hline $66 \times 400$ & 97,3 & 4.55 & 0,52 & 26,14 \\
\hline $116 \times 400$ & 129,2 & 4.31 & 0,69 & 36,7 \\
\hline $204 \times 400$ & 93,6 & 1,47 & 0,27 & 25,02 \\
\hline $102 \times 50$ & 43.5 & 4,9 & 0.01 & 0,03 \\
\hline $202 \times 50$ & 79.2 & 4,46 & 0,01 & 0,03 \\
\hline $202 \times 100$ & 88.1 & 2,48 & 0.02 & 0,32 \\
\hline $402 \times 50$ & 77.3 & 4,23 & 0.01 & falhou \\
\hline $402 \times 100$ & 133.7 & 2,24 & 0,03 & 0,62 \\
\hline $402 \times 200$ & 192.1 & 1.24 & 0,14 & 6,38 \\
\hline
\end{tabular}

Tabela 6.34 - Comparaçào entre o dual canalizado esparso e denso usando o gerador bloco angular

Comparação do tempo de resolução $(n>m)$

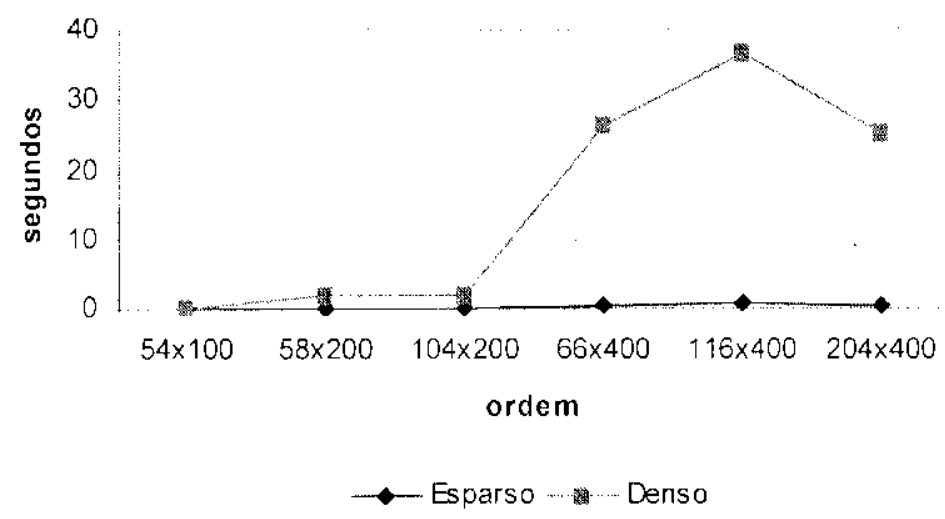

Figura 6.32 - Comparação entre o dual canalizado esparso e denso usando o gerador bloco angular

\section{Comparaçăo do tempo de resolução $(m>n)$}

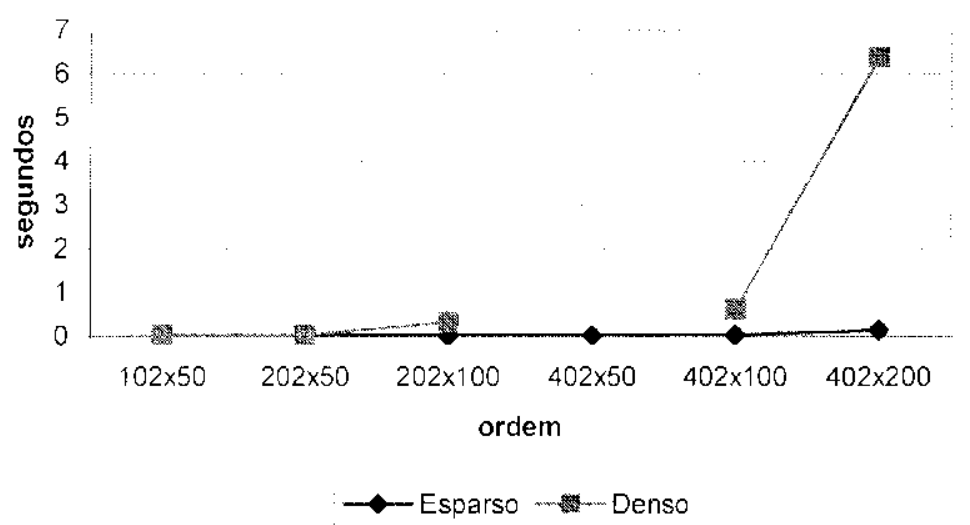

Figura 6.33 - Comparaçào entre o dual canalizado esparso e denso usando o gerador bloco angular 
Para os problemas cuja matriz dos coeficientes está na forma bloco angular, pela tabela 6.34 e liguras 6.32 c 6.33 observamos novamente que o método dual canalizado que trata a esparsidade foi mais rápido que o método dual canalizado que não se preocupa com ela. A diferença entre eles foi maior para os problemas onde $n>m$.

\begin{tabular}{|c|c|c|c|c|}
\hline \multicolumn{5}{|c|}{ Escada } \\
\hline \multirow[b]{2}{*}{ Problemas } & \multirow[b]{2}{*}{ Iteraçöes } & \multirow[b]{2}{*}{$\% \mathrm{nz}$} & \multicolumn{2}{|c|}{ Tempo de resolução } \\
\hline & & & Esparso & Denso \\
\hline $50 \times 101$ & 49.7 & 4,95 & 0.02 & 0,22 \\
\hline $50 \times 201$ & 62.9 & 4.48 & 0.03 & 1,92 \\
\hline $100 \times 201$ & 102.3 & 2.49 & 0,09 & 3,22 \\
\hline $50 \times 401$ & 64,5 & 4.25 & 0.14 & 18,62 \\
\hline $100 \times 401$ & 121.3 & $2: 24$ & 0.34 & 34,89 \\
\hline $200 \times 401$ & 223,1 & 1.25 & 0.66 & 63,95 \\
\hline $100 \times 51$ & 66,2 & 5,88 & 0.01 & 0,05 \\
\hline $200 \times 51$ & 86,2 & 5,88 & 0,02 & 0,08 \\
\hline $200 \times 101$ & 131.4 & 2,97 & 0.03 & 0,58 \\
\hline $400 \times 51$ & 96.7 & 5,88 & 0,02 & 0,08 \\
\hline $400 \times 101$ & 163,5 & 2,97 & 0,05 & 0,78 \\
\hline $400 \times 201$ & 247,3 & 1,49 & 0.2 & 8.33 \\
\hline
\end{tabular}

Tabela 6.35 - Comparação entre o dual canalizado esparso e denso usando o gevador escada

\section{Comparação do tempo de resolução $(n>m)$}

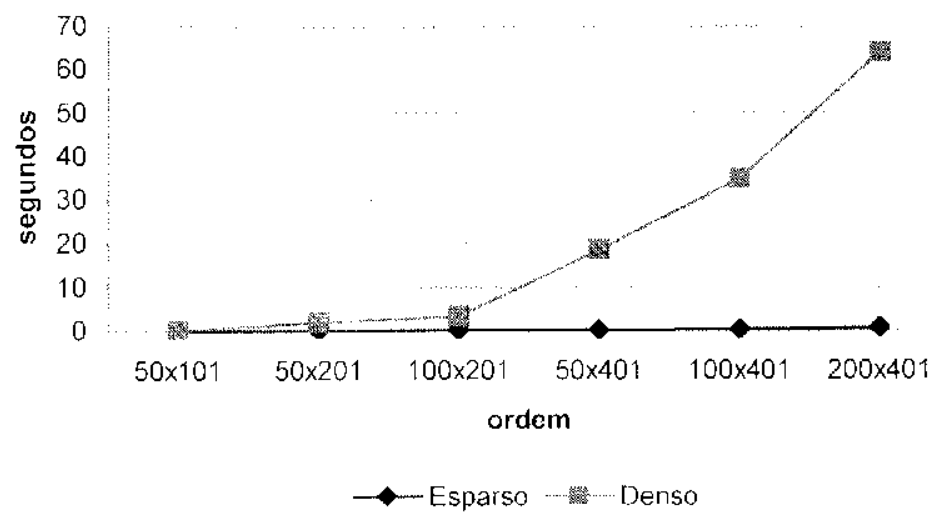

Figura 6.34 - Comparaçăo entre o dual canalizado esparso e denso usando o gerador escada 
Comparação do tempo de resolução $(m>n)$

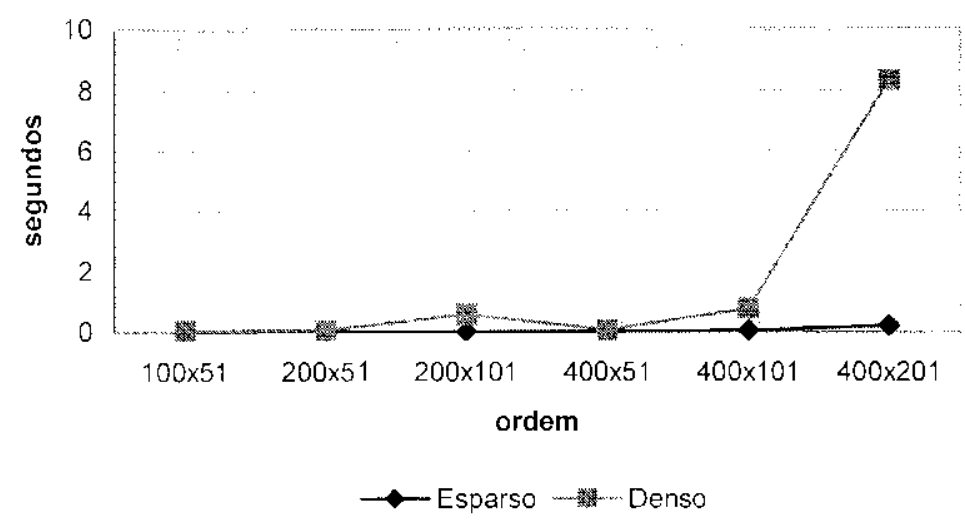

Figura 6.35 - Comparaçâo entre o dual canalizado esparso e denso usando o gerador escada

De acordo com a tabela 6.35 e as figuras 6.34 e 6.35 , os problemas cuja matriz dos coeficientes tem a forma escada foran resolvidos em um tempo muito menor pelo método dual canalizado que explora a esparsidade, chegando em alguns exemplos a ser aproximadamente 100 vezes mais rápido que o método que não explora a esparsidade.

\begin{tabular}{|c|c|c|c|c|}
\hline \multicolumn{5}{|c|}{ Bloco Angular em vários níveis } \\
\hline \multirow[b]{2}{*}{ Problemas } & \multirow[b]{2}{*}{ Iteraçōes } & \multirow[b]{2}{*}{$\% \mathrm{nz}$} & \multicolumn{2}{|c|}{ Tempo de resolução } \\
\hline & & & Esparso & Denso \\
\hline $57 \times 108$ & 36.5 & 1,58 & 0,01 & 0.19 \\
\hline $75 \times 200$ & 72,9 & 1,68 & 0.09 & 1.91 \\
\hline $100 \times 192$ & 70.8 & 1.58 & 0,08 & 2.33 \\
\hline $110 \times 400$ & 145,3 & 1,76 & 0.97 & 46.84 \\
\hline $160 \times 400$ & 117.1 & 1,66 & 0.48 & $44: 32$ \\
\hline $191 \times 400$ & 153.7 & 1,6 & 0,59 & 4184 \\
\hline $105 \times 50$ & 30,2 & 2.15 & 0,01 & 0,02 \\
\hline $194 \times 48$ & 31,9 & 2,15 & 0,01 & 0,02 \\
\hline $220 \times 100$ & 56,3 & 2,29 & 0.02 & 0.23 \\
\hline $402 \times 72$ & 57,1 & 1.66 & 0,02 & 0.12 \\
\hline $431 \times 150$ & 113,4 & 1.52 & 0,11 & 1.71 \\
\hline $433 \times 200$ & 130,5 & 1,86 & 0,19 & 4.14 \\
\hline
\end{tabular}

Tabela 6.36 - Comparação entre o dual canalizado esparso e denso usaudo o gerador bloco angular em vários níveis 


\section{Comparaçäo do tempo de resoluçäo $(n>m)$}

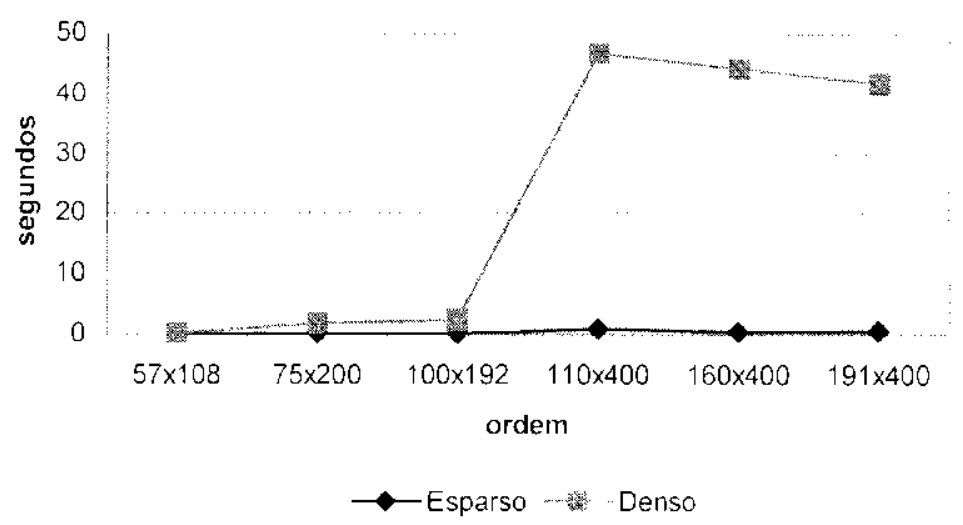

Figura 6.36 - Comparaçào entre o dual camalizado esparso e denso usando o gerador bloco angular em vários niveis

\section{Comparação do tempo de resolução $(m>n)$}

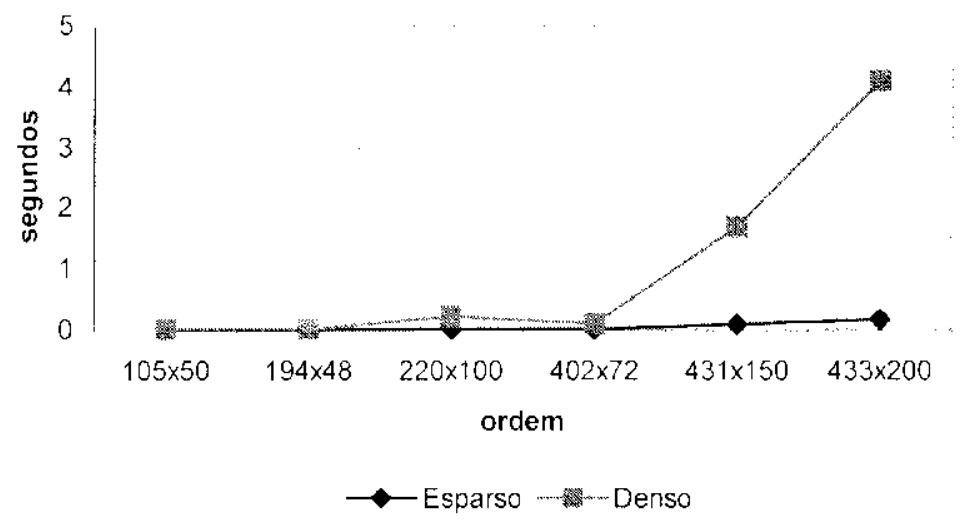

Figura 6.37 - Comparaçào entre o dual canalizado esparso e denso usando o gerałor bloco angutar em vários níveis

Analisande a tabela 6.36 e lïguras 6.36 e 6.37 . podemos perceber que os problemas construidos pelo gerador bloco angular em vários niveis também levou menos tempo para ser resolvido pelo método dual que explora a esparsidade. A diferença entre os métodos foi mais significante para os problemas maiores.

Após todos estes experimentos computacionais realizados, no capítulo 7 serão descritas algumas conclusões e oferecidas algumas sugestões para continuidade e melhoria deste trabalho. 


\section{Capítulo 7 - Conclusões e Propostas Futuras}

\subsection{Conclusões}

Neste trahalho estudamos basicamente os problemas de otimização linear canalizados e esparsos representados de maneira mais geral pela forma (2) e como resolvê-los utilizando o método dual simplex canalizado com busca linear por partes [1], uma ver que este é um método especializado para problenas de otimização linear canalizados e apresentou resultados melhores que o software CPLEX em relação ao número de iterações para problemas densos de acordo com Sousa [37]. Para evitar o cálculo direto da inversa da matriz básica em cada iteraçào, foram estudadas a forma produto da inversa e a decomposição L, J proposta por Bartels-Golub [2]. cuja ênfase está na estabilidade numérica. Mas isto ainda não era suliciente. pois havia a necessidade de se explorar a esparsidade presente nos problemas. Assim, estudamos o algoritmo forma eliminação da inversa, proposto por Forrest c Tomlin | 1.51 e duas variantes do algoritmo de Bartels-Colub propostas por Reid [32]. os quais além de tentar manter a esparsidade existente, apresentam formas de atualizar a decomposição 1.) da matriz básica nas itcraçōes seguintes, enquanto isto for conveniente. $\Lambda$ s variantes ainda apresentam alguns critérios de estabilidade numérica que devem ser usados para evitar que os eros de arredondamento se propaguen muito. I: finalmente, estudamos também as heuristicas de pivoteamento: Crau Mínimo e Markowitz. importantes para evitar que os preenchimentos excessivos ocorram.

Terminado o estudo teórico, o proximo passo foi implementar o método dual simplex canalizado com busca linear por partes juntamente com as duas variantes do algoritmo de Bartel-Golub e também as duas heuristicas de pivoteamento. Para fazer os experimentos computacionais foram implementados quatro geradores. cada um construindo problemas de otimização lincar com estruturas matriciais diferentes. 
Considerando os resultados obtidos com os quatro geradores (produção, escada, bloco angular e bloco angular em vários níveis) em ambos os casos onde $n>m$ e $m>n$. pudemos constatar que houve uma considerável diferença em relação ao número de preenchimentos cutre as combinações da heuristica de Markowitz com as variantes 1 e 2 e as combinaçoes da heurística de (irau Mínimo com as variantes $1 \mathrm{e} 2$, sendo que as duas primeiras combinações aprescntaram resultados melhores e mais estáveis, realizando menos preenchimentos e sem diferenças significantes entre as ordens dos problemas (capitulo 6. seção 6.5).

I m todos os testes realizados vimos que o tempo total de resolução dos problemas utilizando o mélodo dual simplex canalizado foi maior para os problemas no caso em que $n>m$. Isto ocorreu porque o número de preenchimentos feitos também foi maior para este caso. Assim. fica claro o quanto é importante manter a esparsidade para reduzir o tempo de resolução do problema.

1 importante dizer que. conforme os resultados obtidos no capítulo 6, o número de atualizações da decomposição LU realizadas nas iterações seguintes a ela, influenciam bastante no tempo de resolução dos problemas. nos preenchimentos ocorridos e até mesmo no número de iteraçôs. () valor 100 teve um melhor desempenho em geral e valores menores que $\sqrt{m}$ c maiores qu $0.1 \mathrm{~m}$ não apresentam bons resultados.

No comparar o dual simplex canalizado com o CPILXX 7.1, con relação ao tempo de resolução, observamos que para os problemas pequenos a diferença não fó muito significante c o dual chegou a ser vencedor em alguns casos. Porém. para os problemas maiores a diferença aumentou de forma considerável e. principalmente no caso em que $n>m$. a diferença foi muito mais expressiva. Lim se tratando do numero de iterações, podemos dizer que o método dual simplex canalizado lè menos iteraçóes que o (CPL.t.X 7.1 na maioria dos problemas. mas com exceção do gerador produção. para alguns problemas, este fato não ocorrex. conforme os resultados do capítulo 6. A diferença entre o dual simplex canalizado e o CPI.lX 7.1. para o número de iterações. cresceu com a dimensão do problema.

Para finalizar, analisando e comparando os resultados obtidos pelo método dual simplex canalizado que explora a esparsidade. com os resultados obtidos pelo método dual simplex canalizado que não explora a esparsidade e. portanto. trata o problema como se cle fosse denso. vemos que houve uma grande evolução com relação ao tempo de resolução dos problemas do segundo método para o primeiro, onde em alguns exemplos o primeiro método chegou a ser aproximadamente 100 veyes mais rápido e yuanto maior o lamanho dos problemas. a diferença no tempo de resoluçăo entre os métodos também será maior. Assim. podemos dizer que o principal objetivo deste trabalho que era melhorar o desempenho do método já existente explorando a esparsidade foi alcançado. 


\subsection{Propostas futuras}

Trabalhar com esparsidade não é fato novo. Iá algumas décadas tem sido objeto de estudo e. principalmente na última década, grandes evoluções têm se conseguido. Um exemplo disto é o software CPLEX que em 15 anos de sua existência teve um grande progresso entre suas versões. Lim particular. a diferença entre a versão 4.0. usada nos testes feitos por Sousa [37] e a versão 7.1, utilizada neste trabalho, foi significante. Um problema de tamanho $400 \times 200$ leva 17.62 segundos para ser resolvido pela versão 4.0 c apenas 0.06 segundos pela versão 7.1 .

$\Lambda$ implementação do método dual simplex canalizado que trata a esparsidade presente nos problemas, feita neste trabalho, pode ser melhorada acrescentando um "presolve", importante para reduzir o número de equaçoes redundantes e também, otimizando a heuristica de Markowitz., uma vez. que foi constatado que ela apresenta bons resultados realizando menos preenchimentos e, consequentemente, isto faz com que o tempo de resolução seja menor. Fxistem na literatura outras heurísticas de pivoteamento e até mesmo variantes da heurística de Markowit\% que podem também ser implementadas e comparadas.

Ainda com o intuito de melhorar o tempo computacional deste método, pode-se também utilizar outras estruturas de dados, pois através dos testes computacionais realizados percebemos que a estrutura de dados usada nestal implementação é um pouco lenta na inserção dos elementos, principalmente quando a matriz perde a esparsidade.

No método dual simplex canalizado é realizada. em todas as itcrações, a ordenaçâo dos valores que anulam as variáveis hásicas, que são os possíveis tamanhos do passo na direção simplex. Isto está sendo feito através do algoritmo de ordenação "quick sort".

Una sugestão para melhorar o desempenho do algoritmo scria a substituição da rotina de ordenação por:

i) Determine $p_{0}$ tal que $\delta_{j, i}=\min \left\{\delta_{i}\right\}$. Fntão,

Determine $p_{1}$ tal que $\dot{\delta}=\min \left\{\dot{\delta}, \quad i \neq p_{0}\right\}$, etc. atć que $g:-1 \leq 0$.

ii) Determine $p_{0}, p_{1}, \ldots, p_{i+1}$ enquanto $g_{:}>$tolerância.

iii) Determine $p_{0}, p_{1}, p_{i}, l \leq k$, onde $k$ é fixado a priore.

Fistas alternativas surgiram somente após a realização dos experimentos computacionais. onde foi constatado que a ordenação (muitas vezes envolvendo centenas de números) demandava tempo demasiado. 


\section{Referências Bibliográficas}

111 Arenales. M. N. (1984) "Programaçion Linear: Novos Mátodos e Algums Problemas Pariculares". Tese de Doutorado. L nicamp/FFC.

12] Bartels, R. II. (1969) "A Stabilization of the Simplex Method". Numer. Math.. 16. 414-434.

13) Bartels. R. H. and (jolub, (j. H. (1971) "The Simplex Meihod of Linear Programming Using the let Decomposition". Communications of the Association for Computing Machinery 12, 266-268.

IH Bararaa, M. S. et al. (1990) "linear Programming and Netwolk Flow's". Second Idition.

151 Benichou. M. et al. (1977) "The Efficient Solution of Large-Scale Linear Programming Problems - Some Algorithmic Techiniques and C'omputational Results". Math. Programming. 13, 281-322.

16] Bixby. R. 1:. (2001) "Solving Real-World Linear Programs: A Decade and More of l'rogress". II OG. Inc and Rice University.

17) Bixby. R. F. "l al. (1992) "lery Large-Scale Linear Programming: A case Sindy in ('ombining Interior Point and Simplex Methols". Operations Research 40. 885-897.

18] Brayton R. K. Gustavson, F. G. and Willoughby, R. A. (1969) "Some resulls on sparse matrices". IBM Research Centre, Yorklown Heights, N.Y. 
[9] Chen. 1. 1). and Pardalos, P. M. and Saunders M. A. (1994) "The Simplex Algorithm with a New Primal and Dual Pivor Rule", Operations Research I etters 16, 121-127.

110| Dantizg. (i. B. (1963) "Linear Programming and Extensions", Princeton Iniversity Press.

[11] Dantizg. G. B. and Thapa. M. N.(1997) "Linear Programming", Springer.

[12| Dantizg. G. B., Harvey, R. D.. McKnight and Smith S. S.. (1969) "Sparse matrix lechniques in wo mathematical programming codes". Sparse matrix proceedings, Ed. R. Willoughby, RA-I. IBM Research Centre. Yorktown Heights. N.Y.

[1.3] I)uff, 1. S.. Erisman, A. M., Reid, J. K. (1986) "Dired Methods for Sparse Matrices". Clarendon Press. Oxford.

[14] Forrest, J. J and Goldfarb. D. (1992) "Steepest-Edge Simplex Algorithms for Linear Programming", Mathematical Programming 57, 341-374.

[15] lorrest. J. I and Tomlin. J. A. (1972) "Epdated Triangular Factors of the Basis to Maintain Sparsity in the Produet Form Simplex Method, Mathematical Programming 2, 263-278.

[16] (jay, D. M. (1985) "Eletronic Mail Distribution of Linear Programming Test Prohlems:" Mathematical Programming Society COAL Newsletter. December.

[17] Goldfarb, D. and Reid. J. K. (1977) "A Practicable Steepest-Edge Simplex Algorithm". Math. Programming. 12, $361-371$.

[18] Golub, G. E and Van Loan, C. F. (1990) "Matrix Computations". $2^{\text {nd }}$ ed., Johns Hopkins.

[19] Ho. J. K. and loute. I: (198.3) "Computhional Experience with Advanced Implementation of Decomposition Algorithms for Linear Programming", Math. Programming. 27, 283-290. 
120] Kamarkar, ‥(1984) "A New Polynomial Time Algorithm for Linear Programming", Combinatorica. 4, 373-395.

21] Khachian, L. (i. (1979) "A Polynomial Algorithm for Linear Programming", Dokla. Akad. Nauk. USSR. 244, vol 5, 1093-96.

122| Klec. V. and Minty, (. J. (1972) "/how Good is the Simplex Algorithm?", in Shish. O. (Fd.). Proc. 3rd Sympos, Inequalities. AP, Xew York, 159-175.

1231 Lemke, C. E. (1954) "The Dual Method of Solving the Linear Progranming Problem", Naval Research Logistics Quarterly. 1, 36-47.

|24| Lovás\%, L... (1980)"1 New Linear Programming Algorithm - Better or Worse Than The Simplex Method?", Math. Intelligence. 2, vol 3. 141-146.

125| Lustig. I. J. (1989) "An Analisis of an Avable Set of linear Programming Test Problems". (omputers and ()pns. Research 2. vol 16. 173-184.

[26] Markowitz. 1I. M. (1957) "The Elimination Form of the Inverse and its Applications to Linew Programming". Management Science, 3, 255-269.

227| Martin. R. K. (1999) "Iarge Seake Linear and Interger Oprimizalion A unified upproath". Kluwer Academic Publishers.

1281 Megiddo. N. (1991) "On Finding Primal - and Dual - Optimal Bases", ORSAJ. Comp. 3. 63-65.

129) Murty, K. G. (1983)"Linear Programming". The Eniversity of Michigan.

130| Papadimitriou. C. H and Steiglitz, k. (1982) "'(ombinatorial (optimization: Algorithms and ('omplexity". Prentia-l lall.

131| Pissanctzky. S. (1984) "Sparse Marix Technology", Academic Press. London. 
1321 Reid. J. K. (1982) "A Sparsity-Exploiting Variant of the Bartels-(rolub Decomposition for linear Programming Basce". Mathematical Programming 24, 55-69.

1331 Rose, D. I. and Willoughby. R. A (1971) "Sparce Marrices and Applications", Plenum Press. New York.

134| Rosen. J. B.(1990) "Supercomputers and Large-Scale Optimization", Baltzer AJ, Basel.

1351 Saunders, M. A. (1976) "The Complexity of LU Updating in the Simplex Method". R. S. Anderssen and R.P. Brent. eds., "The complexity of computacional problem solving". University Press, Queensland. 214-230.

1361 Shamir, R. (1987) "The lifficiency of the Simplex Method: A Survey". Management Science 3, 301-334.

137| Sousa. R. S. (2000) "Extudos em Otimização Linear". Dissertação de Mestrado, USP Sĩo Carlos - ICMC.

1381 Stern. J. M. (1994) "Esparsidade. Estrutura. Estabilidade e Escalonamento em Álgebra Linear Compuacional", IX Escola de Computação. Recife.

139| Suhl, U. H. and Suhl L. M. (1990) "Computing Sparse (LU) Factorizations for LargeScale Linear Programming". ORSA Journal on Computing 2. 325-335.

140| Terlaky, T. and Zhang. S. (1993) "Pivot Rules for Linear Programming: A Survey on Recent Theoretical Developments", Annals of Operations Research 46, $203-233$.

141| Vanderbei. R. J.(1997) "Linear Programming: Fonndations and Extensions:", Kluwer Academic Publishers. 